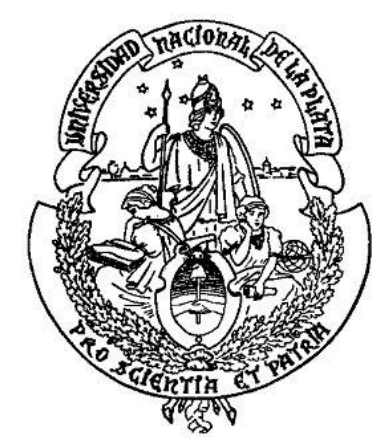

\title{
La dualidad gauge/gravedad y sus aplicaciones a la Materia Condensada
}

\author{
Lic. Ignacio Salazar ${ }^{\dagger}$ \\ Tesis presentada para obtener el titulo de Doctor en Ciencias Exactas \\ ${ }^{\dagger}$ Instituto de Fúsica de La Plata \\ Universidad Nacional de La Plata \\ peznacho@gmail.com
}

Dirigida por el Dr. Nicolás E. Grandi 


\section{Resumen}

La presente tesis doctoral trata sobre recientes contribuciones en el contexto de superfluidos holográficos. Esto significa intentar preguntar y responder cuestiones relevantes acerca de superfluidos fuertemente acoplados en el contexto de la conjetura de AdS/CFT. Siendo así, investigamos agujeros negros cargado y campos en un espacio asintóticamente AdS que consideramos buenos candidatos a duales gravitatorios de un superfluido. Luego usando el diccionario holográfico vemos qué fenomenología obtenemos para los material que viven en el borde de AdS y esperamos que dicha fenomenología sea relevante.

La tesis se organiza de la siguiente manera: Como toda tesis, comienza con una breve introducción al tema, donde se explican conceptos básicos de la conjetura de Maldacena y el Diccionario escrito por Gubser, Klebanov, Polyakov y Witten. Además en dicha introducción se habla sobre algunos de los desafíos actuales en el contexto de materia condensada y sobre cómo la holografía podría ayudar a superar algunos de estos desafíos. Luego se procede a narrar las contribuciones originales del autor al tema, que resumiremos a continuación.

En el capítulo 2 se comenta el trabajo fundacional en el tema, por Hartnoll, Herzog y Horowitz para luego ir un paso más allá y estudiar el caso desordenado. El desorden se logra mediante considerando un potencial químico con una dependencia espacial aleatoria. Luego en el capítulo 3 se estudia la Entropía de entrelazamiento de un modelo con parámetro de orden vectorial. Extensión natural de estos dos modelos, imaginamos un superfluido holográfico con simetría $U(2)$, que nos acompañará desde el capítulo 4 en adelante. De este último modelo estudiaremos conductividades, modos quasinormales, y su comportamiento ante otras fuentes externas, como la velocidad o un segundo potencial químico. 


\section{Agradecimientos}

A Jack.

A Dios, por venir ahora también en sabor limón. A MVC, por ser una magnánima capitana. A mi familia y amigos por aguantarme. Al G7, por su imponente presencia. A Nico, por ser mi maestro Jedi durante este período. A Guille, por aceptarme de Padawan en el CONICET. A Daniel y Antonello, por iluminarme en el ICTP. Al Raulo, por enseñarme que así es el Rock. También a mis otros colaboradores: Amadeo, Isabel, Karl, Luis, Arya y Leo por compartir la grata tarea de investigar. A mis compañeros y amigos platenses y triestinos. A Lord Byron, Castillo, Paul Torrío y el FROGS en general, por su increíble épica. A Barack, por profundizar el concepto de radar. A MOEBIUS, por recordarme cuan divertidos pueden ser los espacios compactos con topología trivial si están volando. Al HIPM, por recordarme que nuestra patria es el mundo. 


\section{Índice general}

$\begin{array}{ll}\text { 1. Introducción } & 7\end{array}$

1.1. Materia Condensada . . . . . . . . . . . . . . . . . 7

1.1.1. ¿Por qué Cond-Mat? . . . . . . . . . . . 7

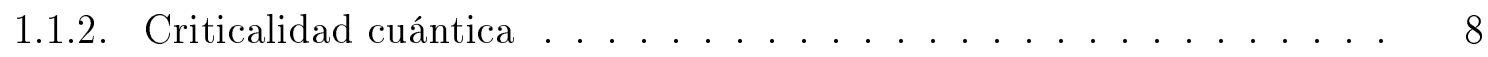

1.1.3. Ejemplo: Lifshitz-Chern-Simons . . . . . . . . . . . . . . . . 8

1.1.4. Superconductores de alta $T_{c} \ldots \ldots \ldots \ldots 11$

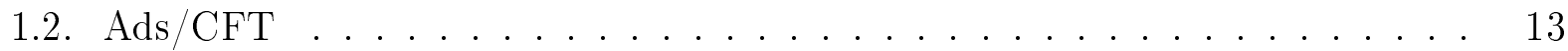

1.2.1. $\mathrm{AdS}=\mathrm{CFT} \ldots \ldots \ldots \ldots \ldots \ldots$

1.2.2. El diccionario . . . . . . . . . . . . . . . . . . . . . . . . . . . . . . . . . . .

1.2.3. Superfluidos holográficos . . . . . . . . . . . . . 15

2. Superfluidos holográficos $\quad 17$

2.1. Superfluidos tipo $s \ldots \ldots \ldots \ldots \ldots \ldots \ldots \ldots$

2.1.1. El modelo: Operadores cargados que condensan . . . . . . . . . . . 17

2.2. Un superconductor holográfico sucio . . . . . . . . . . . . . . . . . . . . . . . . . . . . . . . .

2.2.1. Introducción . . . . . . . . . . . . . . . . . . . 20

2.2.2. Superconductor holográfico sucio . . . . . . . . . . . 21

2.2.3. Introduciendo el desorden . . . . . . . . . . . . . . . . . . . . . . . . . . . . . 22

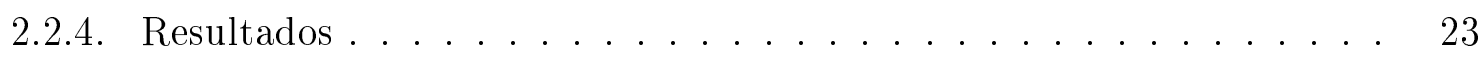

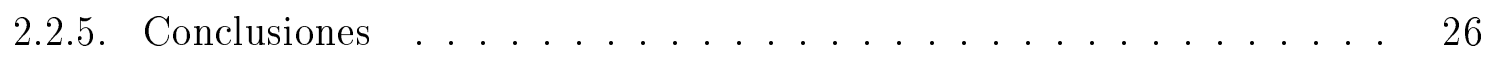

3. Superconductores tipo $\mathrm{p} \quad 29$

3.1. Superconductores tipo $p$ y $p+i p \ldots \ldots \ldots \ldots$

3.1.1. Superconductor tipo $p$ en $2+1$ dimensiones . . . . . . . . . . . . . . . 31

3.1.2. Superconductores tipo $p+i p \ldots \ldots . \ldots . \ldots . \ldots 36$

3.2. Entropía de entrelazamiento . . . . . . . . . . . . . . . 39

3.2.1. Conclusiones . . . . . . . . . . . . . . . . . . . . 42

4. Bosones de Goldstone tipo II holográficos $\quad 45$

4.1. Introducción . . . . . . . . . . . . . . . . . . . 45

4.2. Un modelo de teoría de campos con bosones de Golsdtone de tipo II . . . . . 49

4.3. El modelo no gaugeado . . . . . . . . . . . . . . . . . . . . . . . . . . . . . . . .

4.3.1. Modo de sonido . . . . . . . . . . . . . . . 57

4.3.2. Modo pseudodifusivo . . . . . . . . . . . . . . . . . 58

4.3.3. Modos de Goldstone tipo II . . . . . . . . . . . . . . . . . 60

4.4. El modelo gaugeado . . . . . . . . . . . . . . . . . . 61 
4.4.1. Densidad de carga en la fase rota . . . . . . . . . . . . 63

4.4.2. Fluctuaciones del modelo gaugeado . . . . . . . . . . . . . . . 64

4.4.3. Conductividades . . . . . . . . . . . . . 68

4.4.4. Conductividades en el sector $(0)-(3) \ldots \ldots \ldots 68$

4.4.5. Conductivities in the $(1)-(2)$ sector . . . . . . . . . . 69

4.4.6. Modos quasinormales . . . . . . . . . . . . . . 73

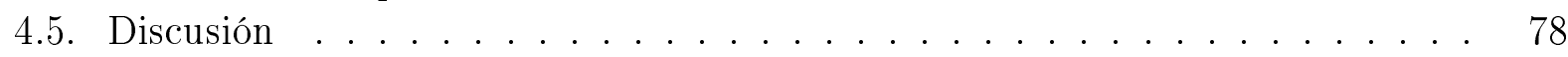

5. Superconductores $\mathrm{s}+\mathrm{p}$ holográficos $\quad 83$

5.1. Introducción . . . . . . . . . . . . . . . . . . 83

5.2. El superfluido holográfico de dos componentes . . . . . . . . . . . . . 85

5.3. El superconductor holográfico $\mathrm{s}+\mathrm{p}$-wave . . . . . . . . . . . 86

5.4. Superconductores desbalanceados . . . . . . . . . . . . . 89

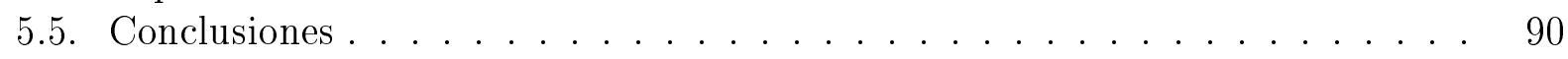

6. Superfluidos holográficos y el criterio de Landau 93

6.1. Introducción . . . . . . . . . . . . . . . . . . . . . . . . . .

6.2. Repaso sobre la teoría de Landau-Ginzburg . . . . . . . . . . . . . . . . . . 96

6.3. El superfluido $U(2)$ con superflujo . . . . . . . . . . . . . . . . . 99

6.3.1. Energía libre . . . . . . . . . . . . . . . 102

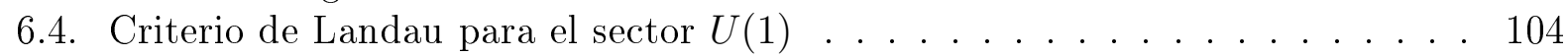

6.4.1. Conductividades longitudinales en el sector $U(1) \ldots \ldots$. . . . . 108

6.5. Criterio de Landau para bosones de Goldstone tipo II . . . . . . . . . . . . . . 110

6.5.1. El modelo Bivio . . . . . . . . . . . . . . . . . . . 112

6.6. Conclusiones . . . . . . . . . . . . . . . . . 113

$\begin{array}{ll}\text { 7. Palabras finales } & 115\end{array}$

$\begin{array}{ll}\text { A. Sobre bosones de Goldstone tipo II } & 117\end{array}$

A.1. Relaciones de Kramers-Kronig matriciales . . . . . . . . . . . . . . . 117

A.2. Resolviendo las ecuaciones para las fluctuaciones . . . . . . . . . . . . . 119

B. Sobre el criterio de Landau $\quad 121$

B.1. Ecuaciones para las fluctuaciones del sector $(0)-(3) \ldots \ldots$. . . . . . 121

B.2. Ecuaciones para las fluctuaciones en el sector $(1)-(2) \ldots \ldots$. . . . 122 


\section{Capítulo 1}

\section{Introducción}

"The first rule of Fight Club is: you do not talk about Fight Club. The second rule of Fight Club is: you DO NOT talk about Fight Club!"

Fight Club

En este capítulo haremos una introducción al tema, siguiendo los lineamientos de [1, 2]

\subsection{Materia Condensada}

\subsection{1. ¿Por qué Cond-Mat?}

Hace décadas que la comunidad cuerdista está buscando algún experimento que pueda ser descripto a través de la teoría de cuerdas. Maldacena parece habernos acercado un paso, a través de su famosa conjetura[3]:

"Type IIB string theory on $\left(A d S_{5} \times S_{5}\right)_{N}$ plus some appropriate boundary conditions (and possibly also some boundary degrees of freedom) is dual to $\mathcal{N}=4$ $d=3+1 U(N)$ super-Yang-Mills".

Ya con las primeras entradas del diccionario a disposición $[4,5]$ surgió la pregunta: ¿Existe en la realidad algún sistema con un dual gravitatorio? Esta pregunta lleva más de una década sin respuesta, pero quizás los sistemas de materia condensada ofrezcan un buen repertorio donde buscar.

Se supone que el modelo estándar y sus extensiones corresponden a una descripción única y completa de nuestro universo. Si es ese el caso, la cromodinámica cuántica $(Q C D)$ podrá tener o no un dual gravitatorio y una vez contestada esa pregunta, no habrá más que hacer ${ }^{1}$. Por el contrario, en materia condensada hay una enorme variedad de hamiltonianos efectivos y muchos nuevos compuestos con nuevas propiedades aparecen cada día. Más aún, jexiste la posibilidad de construir hamiltonianos a través de redes ópticas! Siendo así, éste parece ser un buen escenario donde algún sistema con dual gravitatorio pueda aparecer.

Ahora bien, ¿qué ganaría la comunidad de materia condensada si así fuese? AdS/CFT es una dualidad del tipo "weak-strong", es decir que relaciona una teoría con acoplamiento

\footnotetext{
${ }^{1}$ Esto no implica que la investigación en modelos holográficos de $Q C D$ no sea interesante. Por el contrario, predicciones muy interesantes surgieron en paralelo y casi en simultáneo de los modelos de Sakai-Sugimoto[6] y AdS/QCD [7].
} 
débil a otra con acoplamiento fuerte. Siendo ese el caso, uno podría calcular coeficientes de transporte y otras magnitudes físicas que por el momento no se saben calcular en teorías de campos fuertemente acopladas. Por el momento, el enfoque más exitoso para lidiar con teorías fuertemente acopladas consiste en discretizar el sistema y simular el problema en una red. Sin embargo dicho método se vuelve muy complicado al enfrentarse contra sistemas a densidad finita o fuera del equilibrio.

Desde un punto de vista más filosófico, AdS/CFT nos da una nueva visión sobre las teorías de campos, y es por eso que es un escenario que merece ser investigado. Si la gravedad cuántica puede ser dual a una teoría de campos con muchos aspectos en común con electrones críticos cuánticos, ¿qué importa qué descripción es más fundamental?

\subsubsection{Criticalidad cuántica}

Las transiciones de fase cuánticas son aquellas que ocurren a temperatura $T=0$, a medida que movemos algún parámetro de control del sistema, como puede ser un campo externo o la presión. En estos casos, las transiciones de fase son disparadas por fluctuaciones cuánticas del sistema. Dos fases diferentes a $T=0$ estarán separadas por un punto crítico cuántico.

Típicamente, a medida que nos acercamos a un punto crítico cuántico (QCP), la energía mínima para crear una excitación (el gap de masas) se hace cero, y la longitud de correlación diverge porque el sistema se vuelve invariante de escala. Genéricamente la longitud y la energía no necesitan estar inversamente relacionados. Siendo así, el QCP estará caracterizado por la siguiente invariancia de escala

$$
t \rightarrow \lambda^{z} t, \quad x \rightarrow \lambda x
$$

$z$ es el exponente de Lifshitz (o exponente crítico dinámico) y en general varía de material en material (o en distintos puntos críticos del mismo material). En la próxima subsección veremos un ejemplo con $z=2$, si bien en la naturaleza aparecen los más variados escaleos, incluyendo casos con $z$ no entero. Esta invariancia de escala es la que nos hace intuir que sistemas con puntos fijos cuánticos pueden ser buenos candidatos para tener duales holográficos.

Por el momento parecería que éste es un problema puramente académico, ya que nunca lograremos conseguir $T=0$ en un laboratorio. Sin embargo, las fluctuaciones cuánticas pueden gobernar el diagrama de fases de un sistema siempre que la escala de energías característica, el gap de masas, sea mayor que la temperatura.

Para una visión más amplia acerca de transiciones de fase cuánticas se recomienda [8].

\subsubsection{Ejemplo: Lifshitz-Chern-Simons}

La teoría de Lifshitz-Chern-Simons [9] está definida por la densidad lagrangiana

$$
\begin{array}{r}
S=\int d^{2} x d t \frac{1}{g^{2}}\left[e_{i} \partial_{t} a_{i}+a_{t} \partial_{i} e_{i}-\frac{\kappa^{2}}{2}\left(\partial_{i} e_{j}\right)^{2}-\frac{1}{2} b^{2}-\frac{m^{2}}{2}\left(e_{i}\right)^{2}-\frac{\lambda}{4}\left(e_{i}\right)^{4}\right]+\frac{k}{2} \epsilon_{\mu \nu \lambda} a_{\mu} \partial_{\nu} a_{\lambda}(1.2) \\
\text { con } \quad b[a]=\epsilon^{i j} \partial_{i} a_{j}
\end{array}
$$


Cuando $m^{2}=\lambda=0$, esta teoría presenta invariancia de Lifshitz con $z=2$ y describe por lo tanto un punto crítico. Más aún, si ponemos el nivel de Chern-Simons $k=0$, puede mostrarse que la teoría es el dual electromagnético de un campo escalar de Lifshitz [10].

El operador $e_{i}^{2}$ resulta dominante en el límite IR y su acoplamiento debe ser ajustado cuidadosamente en cero si se desea poner al sistema en el punto de Lifshitz. Si el coeficiente que lo acompaña es positivo, podemos ignorar el término $\left(\partial_{i} e_{j}\right)^{2}$. Uno puede integrar el campo eléctrico recuperando la teoría de Maxwell con «velocidad de la luz» determinada por el coeficiente que acompaña a $e_{i}^{2}$. Si en cambio el coeficiente que acompañaśa $e_{i}^{2}$ es negativo, un término de orden más alto, como $\left(e_{i}^{2}\right)^{2}$ debe estar presente con signo positivo para estabilizar la teoría y tener energías acotadas por abajo. En este caso, el sistema puede presentar ruptura espontánea de simetría rotacional. Este tipo de términos se hace irrelevante si $e_{i}^{2}$ es acompañado por un coeficiente positivo. En caso de que $\left(e_{i}^{2}\right)^{2}$ venga acompañado por un coeficiente negativo, sería necesario agregar un término $\left(e_{i}^{2}\right)^{3}$ para estabilizar el sistema, pero no consideraremos dicho caso.

Entonces tenemos una transición de fase cuántica entré un estado Hall cuando $m^{2}>0 \mathrm{y}$ un estado anisotrópico cuando $m^{2}<0$ separadas por un punto crítico de Lifshitz. Veamos ahora qué características presentan al ser excitados con una fuente externa

\section{Coeficientes de transporte}

Se suele construir una teoría de Chern-Simons alrededor de la existencia de una corriente conservada, la cual permite inmediatamente definir un campo de gauge de la siguiente manera:

$$
J^{\mu}=\frac{1}{2 \pi} \epsilon_{\mu \nu \rho} \partial_{\nu} a_{\rho}
$$

Una vez definido, el lagrangiano para el campo $a_{\rho}$ será el discutido más arriba, por ser aquel que respeta la simetría de escaleo con $z=2$.

Vale la pena preguntarse como se comportará el sistema, frente a la acción de un campo externo de gauge $A^{\mu}$ que se acople mínimamente a la corriente conservada, de manera tal que

$$
\delta S=\int d^{2} x d t A^{\mu} J_{\mu}
$$

A orden cuadrático en la acción, podemos integrar para escribir una acción efectiva para el campo externo. La acción completa tiene la forma

$$
S=\int d^{2} x d t\left(\frac{1}{2} O_{m n} æ_{m} æ_{n}+J_{\rho} A^{\rho}\right) .
$$

donde $m, n=0, \ldots, 4, x_{m}=a_{m}$ para $m=0,1,2$ y $x_{m}=e_{m-2}$ para $m=3,4$ y $\mu, \nu, \rho=$ $0, \ldots, 3$. La forma del operador $O_{m n}$ se puede leer de la expresión (1.2). Siendo así, podemos encontrar la siguiente ecuación de movimiento para la transformada de Fourier

$$
O_{\mu n}\left(i \omega_{n}, \vec{p}\right) æ_{n}\left(i \omega_{n}, \vec{p}\right)-\frac{1}{2 \pi} \epsilon_{\lambda \rho \mu} i p_{\rho} A^{\lambda}\left(i \omega_{n}, \vec{p}\right)=0
$$


que se puede reescribir usando la definición del campo æ y de la corriente $J$ :

$$
\begin{aligned}
J_{\alpha}\left(i \omega_{n}, \vec{p}\right) & =\frac{1}{2 \pi} \epsilon_{\alpha \beta \nu} i p_{\beta} a_{\nu}\left(i \omega_{n}, \vec{p}\right)= \\
& =-\frac{1}{4 \pi^{2}} O_{\mu \nu}^{-1}\left(i \omega_{n}, \vec{p}\right) \epsilon_{\alpha \beta \nu} \epsilon_{\lambda \rho \mu} p_{\beta} p_{\rho} A^{\lambda}\left(i \omega_{n}, \vec{p}\right) .
\end{aligned}
$$

donde

$$
O_{\alpha \beta}^{-1}=\left\langle a_{\alpha}\left(-i \omega_{n},-\vec{p}\right) a_{\beta}\left(i \omega_{n}, \vec{p}\right)\right\rangle
$$

La misma ecuación de movimiento se puede obtener de la siguiente acción efectiva variándola respecto a $A_{\mu}$

$$
S_{\text {ef }}=\frac{1}{2} \sum_{n} \int d^{2} p A_{\mu}\left(-i \omega_{n},-\vec{p}\right) K_{\mu \nu}\left(i \omega_{n}, \vec{p}\right) A_{\nu}\left(i \omega_{n}, \vec{p}\right)
$$

donde

$$
K_{\mu \nu}\left(i \omega_{n}, \vec{p}\right)=\left(\frac{1}{2 \pi}\right)^{2} \epsilon_{\alpha \beta \nu} \epsilon_{\lambda \rho \mu} p_{\beta} p_{\rho}\left\langle a_{\alpha}\left(-i \omega_{n},-\vec{p}\right) a_{\lambda}\left(i \omega_{n}, \vec{p}\right)\right\rangle
$$

Por otro lado, la definición tradicional de la conductividad eléctrica es

$$
J^{i}=\sigma_{i j} E^{j}
$$

Si además consideramos el caso de momento nulo, tenemos que $E^{i}\left(i \omega_{n}, \vec{p}\right)=i \omega A^{i}\left(i \omega_{n}, \vec{p}\right)$, lo que implica que la conductividad será, según la fórmula de Kubo

$$
\sigma_{i j}=\frac{1}{i \omega} K_{i j}(i \omega, \vec{p}=0)
$$

Como las funciones de Green tendrán polos para ciertos valores de $\omega$, aun queda elegir cómo saltar dichos polos. El cambio $i \omega_{n} \rightarrow \omega+i \delta$ nos asegura que las funciones de Green que utilicemos sean las retardadas. Siendo así, la expresión para la conductividad queda

$$
\sigma_{i j}=\frac{1}{i \omega+\delta} K_{\mu \nu}(\omega+i \delta, \vec{p}=0)
$$

Para calcular la conductividad es necesario en primer lugar calcular los propagadores, para lo cual es necesario fijar un gauge. Nosotros elegimos el gauge $\partial_{i} a_{i}=0$ e hicimos explícita dicha elección agregando un término $(\alpha / 2)\left(\partial_{i} a_{i}\right)^{2}$ en la densidad lagrangiana. Finalmente, tomamos el valor $\alpha=-1$ ya que simplifica los cálculos, y corresponde al gauge de Feynman en la teoría del electromagnetismo común. Veamos cuál es el valor de dicha conductividad en las distintas fases y en el punto crítico.

En el punto crítico, $m^{2}=0$ tenemos

$$
\begin{aligned}
\sigma_{x x} & =0 \\
\sigma_{x y} & =0
\end{aligned}
$$

En la fase isotrópica, $m^{2}>0$ es de esperarse que el comportamiento sea el mismo que para la teoría tradicional de Maxwell-Chern-Simons, ya que el término $e_{i}^{2}$ rompe la invariancia 
de escala con $z=2$, y a bajas energías nuestro lagrangiano y el de Maxwell-Chern-Simon coinciden. Las funciones de respuesta son de hecho

$$
\begin{aligned}
\sigma_{x x} & =\frac{i}{8 \pi^{2} k} \frac{\omega / g^{4} k m^{2}}{1-\left(\omega / g^{4} k m^{2}\right)^{2}} \\
\sigma_{x y} & =\frac{1}{8 \pi^{2} k} \frac{1}{1-\left(\omega / g^{4} k m^{2}\right)^{2}}
\end{aligned}
$$

Vemos que el efecto del término de Chern-Simons es introducir una conductividad Hall en la dirección transversal al campo eléctrico. A frecuencias bajas, la parte real de la conductividad longitudinal se anula y la conductividad Hall se hace $1 / 8 \pi^{2} k$.

En la fase anisotrópica, $m^{2}<0, e_{i}$ desarrolla un valor de expectación, rompiendo así la simetría rotacional $S O(2)$. Suponiendo que no haya winding, podemos elegir al campo de vacío como $e_{i}=\left(\sqrt{-m^{2} / \lambda}, 0\right)$. Entonces tenemos para las conductividades

$$
\begin{aligned}
\sigma_{x x}(\omega) & =0 \\
\sigma_{y y}(\omega) & =\frac{g^{2} m^{2}}{2 \pi} \delta(\omega)+\frac{i g^{2} m^{2}}{2 \pi^{2} \omega} \\
\sigma_{x y}(\omega) & =0 .
\end{aligned}
$$

Esto implica que el sistema es aislante en una dirección y superconductor en la dirección transversal.

La relación entre la parte real e imaginaria de la conductividad está fija por las reglas de Kramers-Kronig

$$
\begin{gathered}
\operatorname{Re} G^{R}(\omega)=P \int_{-\infty}^{\infty} \frac{d \omega^{\prime}}{\pi} \frac{\operatorname{Im} G^{R}\left(\omega^{\prime}\right)}{\omega^{\prime}-\omega} \\
\operatorname{Im} G^{R}(\omega)=-P \int_{-\infty}^{\infty} \frac{d \omega^{\prime}}{\pi} \frac{\operatorname{Re} G^{R}\left(\omega^{\prime}\right)}{\omega^{\prime}-\omega} .
\end{gathered}
$$

A la hora de mirar superconductores holográficos, la delta a $\omega=0$ se escapará al análisis numérico y por ello miraremos el polo en la parte imaginaria para determinar su presencia.

Para hacer estas cuentas, fue fundamental estar a acoplamiento débil, para poder cortar la serie de Dyson a nivel árbol. Cuando estudiemos sistemas holográficos, construiremos modelos de juguete como el acá presentado, usando agujeros negros en AdS. De esta manera vamos a estudiar la fenomenología de la rotura espontánea de simetría a acoplamiento fuerte, que corresponderá a gravedad y campos clásicos en el interior de AdS.

\subsubsection{Superconductores de alta $T_{c}$}

Sistemas de la vida real que quizás puedan tener una descripción efectiva en términos de duales gravitatorios son los superconductores de alta temperatura crítica.

Nos referimos a alta temperatura crítica cuando la misma está por encima de los $30 K$, que es el límite máximo permitido por BCS. Los primeros superconductores de alta $T_{c}$ fueron descubiertos en 1986 y desde entonces una descripción teórica consistente de su comportamiento sigue siendo un tema candente de investigación. Esto se debe principalmente al hecho de que estos sistemas están fuertemente acoplados, lo que hace que los recursos teóricos usuales resulten obsoletos. 
Por lo general, estos materiales tienen una fuerte estructura de capas, lo que hace que la física relevante sea básicamente bidimensional. Inspirados en este hecho, el resto de esta tesis tratará básicamente con sistemas duales a teorías que viven en dos dimensiones espaciales.

Analicemos el diagrama de fases de estos superconductores, que se puede ver en la figura 1.1. Empecemos por el lado derecho, descripto por un líquido de Fermi. Esto significa que en este sector del sistema el efecto de las interacciones es simplemente renormalizar la masa y la carga de las partículas fundamentales. Siendo así existe una buena descripción en términos de quasipartículas "vestidas" con las mismas propiedades que las partículas fundamentales que actúan de portadores de carga en el material. Una característica propia de la teoría de Landau para líquidos de Fermi (también conocidos como líquidos de Landau-Fermi) es su función de Green de dos puntos, caracterizada por el siguiente comportamiento cerca de la superficie de Fermi [12]

$$
G_{R}(\omega, \vec{k})=\frac{Z}{\omega-v_{F}\left(k-k_{F}\right)+\Sigma(\omega, k)+\ldots,}
$$

donde la auto-energía $\Sigma(\omega, k)$ se escribe

$$
\Sigma=\frac{i \Gamma}{2} \approx i \omega^{2} .
$$

El residuo $Z<1$ del polo, que es llamado "peso" de la quasipartícula, puede ser interpretado como la superposición entre el estado de una quasipartícula y el estado generado al hacer actuar el operador que crea un electrón sobre el vacío.

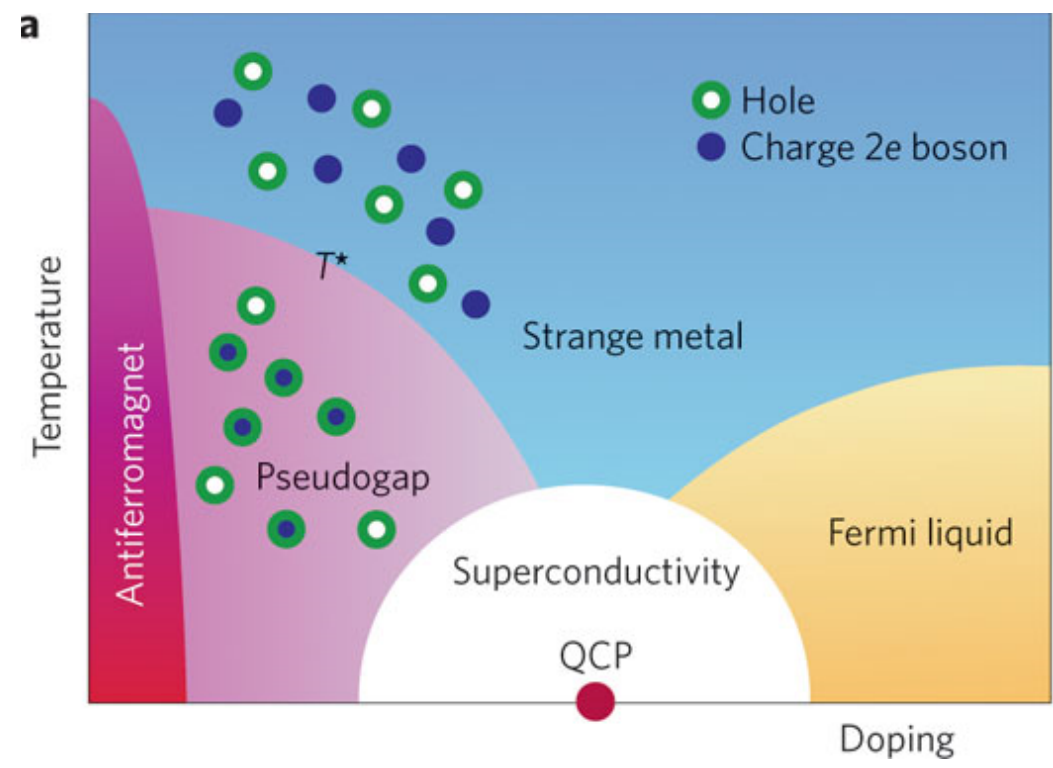

Figura 1.1: Diagrama de fases para los superconductores de alta temperatura crítica tomado de [11]

A medida que bajamos el dopaje (cantidad relativa de impurezas), manteniéndonos todavía a temperaturas altas, hay una transición de fase o quizás un cross over, a una fase 
llamada de metal extraño. El mismo consiste en un sistema de fermiones que no es bien descripto por la teoría de Landau. ¿Cómo se ve fenomenológicamente dicha falla? por lo general se trata de materiales donde el calor específico no crece linealmente con la temperatura, o la resistividad no crece de manera cuadrática, según implicaría la teoría de Landau [12]. En los cupratos por ejemplo, la resistividad crece de manera lineal con la temperatura.

Los experimentos de Angular Resolved Photoemission Spectroscopy (ARPES) arrojan un poco más de luz sobre el origen de estos comportamientos anormales en los cupratos en la fase de metal extraño. Los mismos indican que estos metales poseen superficies de Fermi, pero que el comportamiento no analítico de la función espectral es distinto de aquel predicho para líquidos de Fermi según la teoría de Landau. La función de Green se ajusta entonces a la de un líquido de Landau-Fermi, con la diferencia de que la auto energía viene dada por

$$
\Sigma(\omega) \approx c \omega \log \omega+d \omega,
$$

con $c$ un número real mientras que $d$ es un número complejo. Vemos entonces que el sistema tiene excitaciones no masivas pero ahora la taza de decaimiento $\Gamma$ de dichas excitaciones es proporcional a $\omega$ en lugar de cuadrática como en el caso de líquidos de Landau-Fermi. Una taza de decaimiento de orden $\omega$ es tan grande que una excitación decae antes de propagarse lo suficientemente lejos (i.e. una longitud de onda) como para mostrar sus propiedades de partícula. Como resultado, dicha excitación ya no puede ser tratada como quasipartícula. En resumen, los metales extraños pueden ser sistemas con superficies de Fermi pero sin quasipartículas. Dichos sistemas fueron estudiados por ejemplo en [13, 14, 15, 16, 17].

Si bajamos la temperatura entramos en la fase superconductora. Por el momento se cree que la misma es del tipo $d$ (el parámetro de orden parece ser un tensor de rango dos), aunque al escaparse de la descripción de BCS poco es lo que se conoce al respecto. Un aspecto interesante es que esta fase superconductora se encuentra vecina a una fase ordenada magnéticamente, como puede ser un antiferromagneto. Esto significa que la competencia y coexistencia de distintos órdenes tiene un rol crucial en la comprensión completa del diagrama de fases de estos superconductores.

En esta tesis intentaremos abordar el tema de superfluidez desde el punto de vista de AdS /CFT.

\subsection{Ads/CFT}

En esta sección se dará una breve introducción a la correspondencia AdS/CFT, sin intentar justificarla desde un punto de vista fundamental (en ese sentido se recomienda [18, 19]). En cambio, daremos las entradas básicas del diccionario y cuáles son los elementos mínimos necesarios para buscar duales gravitatorios a sistemas de materia condensada.

\subsection{1. $\quad$ AdS $=$ CFT}

La expresión efectiva mínima posible de AdS/CFT es que las teorías de gauge en el límite de $N$ grande en $d$ dimensiones se corresponden con sistemas de gravedad clásica en $d+1$ dimensiones. Esta dimensión extra parametriza la escala de energía de la teoría de campos, permitiendo ver el flujo de renormalización de una manera geométrica.

Cuando se habla del enfoque wilsoniano de una teoría cuántica de campos, la teoría está en general definida por un cut-off ultravioleta (UV) o por un punto fijo ultravioleta. 
Un punto fijo es el lugar más sencillo del diagrama de fases para comenzar a hablar de la correspondencia AdS/CFT. En un punto fijo la teoría es invariante de escala. Por simplicidad asumiremos $z=1$ en (1.1). La dualidad para $z$ arbitrario fue introducida en [20] y es aun un tema activo de investigación.

La lógica de AdS/CFT nos sugiere buscar una métrica en una dimensión más que realice de manera geométrica la simetría conforme de la teoría en un punto crítico. Siendo así, se llega a la métrica de un espacio AdS

$$
d s^{2}=L^{2}\left(-r^{2} d t^{2}+r^{2} d x_{i}^{2}+\frac{d r^{2}}{r^{2}}\right)
$$

que es solución de la teoría más simple de gravedad que uno pueda imaginar: relatividad general con constante cosmológica negativa.

$$
S=\int d^{d+1} x \sqrt{-g}\left(R+\frac{d(d-1)}{L^{2}}\right) .
$$

Uno puede romper la invariancia de escala de esta métrica preservando rotaciones y traslaciones espacio temporales introduciendo factores de ennegrecimiento, que corresponderán a la solución de agujero negro

$$
d s^{2}=L^{2}\left(-f(r) r^{2} d t^{2}+r^{2} d x_{i}^{2}+\frac{d r^{2}}{f(r) r^{2}}\right)
$$

con

$$
f(r)=1-\left(\frac{r_{+}}{r}\right)^{d} .
$$

Esto corresponde a poner la teoría de campos dual a una temperatura finita dada por la temperatura del agujero negro. Esta de obtiene del período de compactificación del tiempo euclídeo, determinado al exigir regularidad cerca del horizonte de la correspondiente extensión euclídea

$$
i t \equiv \tau=\tau+\frac{4 \pi r_{+}}{d}
$$

La temperatura de la teoría conforme dual será entonces

$$
T=\frac{d}{4 \pi r_{+}} .
$$

Por el momento vimos cómo relacionar puntos críticos con espacios AdS y cómo poner la teoría en un baño térmico. Para obtener más detalles al respecto a la fenomenología de la CFT, debemos profundizar un poco más en las entradas del diccionario.

\subsubsection{El diccionario}

Una de las entradas más importantes del diccionario es que un operador $\mathcal{O}$ en la teoría de campos se corresponde a un campo dinámico en el interior de la geometría (bulk), de manera tal que

$$
Z_{\text {bulk }}\left[\phi(\vec{x}, r \rightarrow \infty) \rightarrow \phi_{0}(\vec{x})\right]=\left\langle e^{\int d^{4} x \phi_{0}(\vec{x}) \mathcal{O}(\vec{x})}\right\rangle_{F . T .},
$$

donde $\phi_{0}$ es la condición de contorno para el campo en el bulk. Para sacar información útil de esta prescripción, vale la pena repasar el comportamiento genérico de los campos en AdS. 
Un campo de prueba en AdS se comportará cerca del borde como

$$
\phi(\vec{x}, r) \approx \phi_{+}(\vec{x}) r^{\Delta_{+}}+\ldots+\phi_{-}(\vec{x}) r^{\Delta_{-}}+\ldots
$$

donde $\Delta_{ \pm}$se corresponde con los dos posibles comportamientos de las dos soluciones linealmente independientes de la ecuación de movimiento correspondiente a $\phi$, que supondremos de segundo orden. La prescripción (1.28) nos dice que $\phi_{+}$corresponderá a la fuente del operador dual mientras que $\phi_{-}$al valor de expectación.

Este no es más que un ejemplo esquemático del procedimiento. Por lo general, será necesario añadir contratérminos a la acción, que aparecerán como términos de borde que cancelen las eventuales divergencias (para detalles en este aspecto se recomienda ver [21]). Por otro lado términos de borde pueden aparecer en casos en los que el principio variacional no esté bien definido. Esto ocurre con campos de gravedad y campos fermiónicos [22, 24].

Ahora bien, ¿qué pasa si el campo dinámico $\phi(\vec{x}, r)$ tiene índices asociados? Los índices se corresponderán con índices del operador en el borde. Así, tener escalares en el bulk significa prender operadores escalares en el borde mientras que tener fermiones en el bulk significa prender operadores fermiónicos en el borde. Los campos vectoriales en el bulk se corresponden con corrientes en el borde.

Más aún, una simetría local en el bulk se traduce en la misma simetría pero global en el borde. Esto último es sumamente importante a la hora de construir soluciones de gravedad que intenten representar fenómenos de materia condensada. Por lo general atacaremos el análisis de un sistema à la Landau-Ginzburg, analizando las simetrías del sistema y definiendo transiciones a fases con la simetría rota. En este contexto, mirar transiciones de fase corresponderá a analizar la energía libre de soluciones con el mismo comportamiento cerca del borde (ultravioleta de la teoría de campos) pero diferente comportamiento lejos (infrarrojo de la teoría de campos).

Por otro lado, esta misma prescripción nos permite calcular funciones de $n$ puntos. De particular interés serán las funciones de 2 puntos. Los modos quasinormales (QNM) de un agujero negro en AdS nos dan información de las quasipartículas viviendo en el borde. Así, podemos estudiar las masas y relaciones de dispersión de las mismas y obtener observables de una teoría cuyo lagrangiano desconocemos. En este mismo espíritu, correladores entre gauge fields en el bulk se pueden relacionar con conductividades en el borde, a través de la fórmula de Kubo explicada en la sección 1.1.3

Durante esta tesis se verán varios ejemplos donde se aplican todos estos conceptos.

\subsubsection{Superfluidos holográficos}

En los siguientes capítulos, veremos distintas realizaciones de superfluidos holográficos. En esta sección se pretende dar una respuesta esquemática a la pregunta: ¿a qué nos referimos cuando hablamos de superfluidos holográficos?

Un superfluido será para nosotros un sistema que rompe una simetría global $U(1)$ de manera espontánea al mover alguno de los parámetros del sistema. Traduciendo en el lenguaje de AdS/CFT, los elementos mínimos serán:

- Una geometría con un borde AdS.

- Un campo de gauge $U(1)$ en el bulk para tener simetría global $U(1)$ en el borde. 
- Un campo cargado bajo $U(1)$ en el bulk que tenga soluciones normalizables en la geometría para ciertos valores de los parámetros, que darán origen a valores de expectación que romperán la simetría global $U(1)$ en el borde.

No nos preocuparemos por tener lagrangianos consistentes con teorías de supergravedad (SUGRA), sino que nuestro enfoque será buscar los modelos más simples que presenten este tipo de fenomenología.

\section{Comentario: Sobre remanentes de la simetría conforme, honestidad en las notaciones y realización numérica.}

A lo largo de esta tesis vamos a trabajar con distintos modelos de superfluidos holográficos. A lo largo de la misma la notación irá mutando, así como cambia de publicación en publicación. Este apartado aparece acá con la intención de dar guía a través del promiscuo universo de las notaciones, explicando las verdades físicas y matemáticas que se esconden detrás.

Lo primero que debemos notar es que en una teoría invariante de escalas a temperatura finita y en equilibrio no hay otra escala con la cual podemos comparar la temperatura. Por este motivo, todas las temperaturas diferentes de cero deberían ser equivalentes. Este hecho se ve reflejado en la métrica de Schwarchild-AdS (1.22): el escaleo $\left(r, t, x^{i}\right) \rightarrow r_{+}\left(r, t, x^{i}\right)$ elimina a $r_{+}$de la métrica. En una teoría invariante de escala sólo hay dos temperaturas distinas: cero y no-cero.

Una estructura que suele emerger en sistemas de materia condensada es la simetría $U(1)$. Esto se puede realizar en el bulk agregando campos de gauge al lagrangiano de materia. Cambiando las condiciones de contorno, podemos ahora obtener nuevas soluciones de nuestra teoría correspondientes a agujeros negros con carga. En la teoría del borde esto corresponde a agregar potencial químico y densidad de carga finitos. Desde el punto de vista lúdico, ahora tenemos una perilla que mover y nuestro diagrama de fases va a dejar de ser dos puntos $(T=$ $0, T \neq 0$ ) para agregar ahora todas los posibles potenciales químicos o densidades de carga, dependiendo de si trabajamos en el ensamble gran canónico o canónico respectivamente.

A lo largo de esta tesis vamos a trabajar a temperatura finita en el ensamble gran canónico fijando el potencial químico físico a uno. Esto equivale a medir todo en unidades del potencial químico al cual sometemos la teoría, ya que básicamente la magnitud relevante es ahora el cociente de la temperatura y el potencial químico $T / \mu$.

A la hora de integrar ecuaciones de movimiento sin embargo, lo más cómodo será dejar la posición del horizonte fija. Siendo así, para obtener soluciones a distintos $T / \mu$ moveremos la carga del agujero negro. Para volver a nuestro sistema físico a $\mu=1$ reescalaremos todas las magnitudes que deseemos graficar usando las correspondientes potencias de $\mu$.

Ahora sí, procedemos a hablar en detalle de los superfluidos holográficos. 


\section{Capítulo 2}

\section{Superfluidos holográficos}

\subsection{Superfluidos tipo $s$}

En esta sección explicaremos el modelo primero y más sencillo de un superfluido holográficos, introducido en [27] ${ }^{1}$. La idea del trabajo original era ver si un modelo mínimo podía reproducir aunque sea algunos aspectos de la fenomenología de superconductores no convencionales.

¿Por qué superconductores no convencionales? BCS parece ser un buen modelo microscópico para la gran mayoría de los superconductores. Sin embargo los superconductores de alta temperatura crítica parecen escaparse a esta descripción. De hecho, todavía no se sabe cuál es el mecanismo que produce el apareamiento en estos materiales, y se duda de que un líquido de Fermi sea una buena descripción para su fase normal. En este contexto, quizás AdS/CFT pueda describir líquidos duales lo suficientemente extraños como para poder describir superconductores no convencionales.

En muchos de estos materiales, como los cupratos, gran parte de la física vive en $2+1$ dimensiones. Por eso se trabajará con espacios asintóticamente $A d S_{4}$. Se agregará temperatura a la teoría dual mediante un agujero negro, una simetría global $U(1)$ a través de un campo de gauge y un campo escalar cargado que esperaremos que condense. Para reproducir cualitativamente el diagrama de fases de superconductores, esperamos que aparezcan soluciones normalizables para el escalar sólo a temperaturas por debajo de cierta temperatura crítica $T_{c}$.

\subsubsection{El modelo: Operadores cargados que condensan}

Empezamos con la métrica del agujero negro de Schwarzschild en AdS

$$
d s^{2}=-f(r) d t^{2}+\frac{d r^{2}}{f(r)}+r^{2}\left(d x^{2}+d y^{2}\right)
$$

donde

$$
f=\frac{r^{2}}{L^{2}}-\frac{M}{r}
$$

\footnotetext{
${ }^{1}$ La diferencia entre un superconductor y un superfluido proviene de lo siguiente: en ambos se produce la ruptura espontanea de una simetría pero en el primer caso esta es una simetría local mientras que en el segundo caso es global. En esta tesis vamos a usar indiferentemente una y otra palabra, aunque técnicamente siempre nos refiramos a superfluidos, ya que la simetrías en el borde son globales.
} 
$L$ es el radio de AdS y $M$ determina la temperatura de Hawking del agujero negro:

$$
T=\frac{3 M^{1 / 3}}{4 \pi L^{4 / 3}}
$$

Este agujero negro es $3+1$ dimensional, y va a ser dual a una teoría en $2+1$ dimensiones. En esta geometría, considérese ahora un campo de Maxwell y un escalar cargado, con densidad lagrangiana

$$
\mathcal{L}=-\frac{1}{4} F^{a b} F_{a b}-V(|\Psi|)-|\partial \Psi-i A \Psi|^{2}
$$

Por simplicidad, nos concentraremos en el caso

$$
V(|\Psi|)=-\frac{2|\Psi|^{2}}{L^{2}}
$$

Aunque la masa al cuadrado es negativa, esta elección no produce una inestabilidad, ya que está por arriba de la cota de Breitenlohner-Freedman. Vale aclarar que vamos a trabajar en un límite en el que los campos de Maxwell ni el escalar afectan la métrica.

Tomando un ansatz con simetría planar, $\Psi=\psi(r)$ y real y $A_{t}=\phi(r)$ obtenemos las ecuaciones de movimiento

$$
\begin{gathered}
\psi^{\prime \prime}+\left(\frac{f^{\prime}}{f}+\frac{2}{r}\right) \psi^{\prime}+\frac{\phi^{2}}{f^{2}} \psi+\frac{2}{L^{2} f} \psi=0 \\
\phi^{\prime \prime}+\frac{2}{r} \phi^{\prime}-\frac{2 \psi^{2}}{f} \phi=0
\end{gathered}
$$

En el horizonte $r=r_{0}$ para que $\phi d t$ esté bien definido tenemos que pedir $\phi\left(r_{0}\right)=0$ y la ecuación (2.5) implica que $\psi^{\prime}=-2 \psi / 3 r_{0}$. Entonces, tenemos una familia de dos parámetros de soluciones regulares en el horizonte. En el infinito, estas soluciones se comportan según

$$
\begin{gathered}
\psi=\frac{\psi^{(1)}}{r}+\frac{\psi^{(2)}}{r^{2}}+\ldots, \\
\phi=\mu-\frac{\rho}{r}+\ldots
\end{gathered}
$$

Para $\psi$, los dos decaimientos son normalizables, así que podemos imponer como condición de borde que cualquiera de los dos se anule. Por simplicidad diremos que $\psi^{(1)}$ es la fuente y pediremos entonces que sea el coeficiente que se anule. Luego de imponer esa condición de borde, nos queda una familia de soluciones que depende sólo de un parámetro.

Las propiedades de la teoría de campos dual se pueden leer del comportamiento asintótico de la solución. Por ejemplo, $\mu$ será el potencial químico mientras que $\rho$ será la densidad de carga. El condensado del operador escalar $\mathcal{O}$ en la teoría de campos dual al campo $\psi$ vendrá dado por

$$
\left\langle\mathcal{O}_{2}\right\rangle=\sqrt{2} \psi^{(2)}
$$

A partir de ahora trabajaremos en unidades en las que el radio de AdS es $L=1$. Recuérdese que $T$ tiene dimensiones de masa, así que $\mathcal{O}_{2} / T^{2}$ y $\rho / T^{2}$ serán cantidades adimensionales. Siempre trabajaremos en términos de estas, ya que son las que reproducen la física relevante, dejando de lado escaleos. 


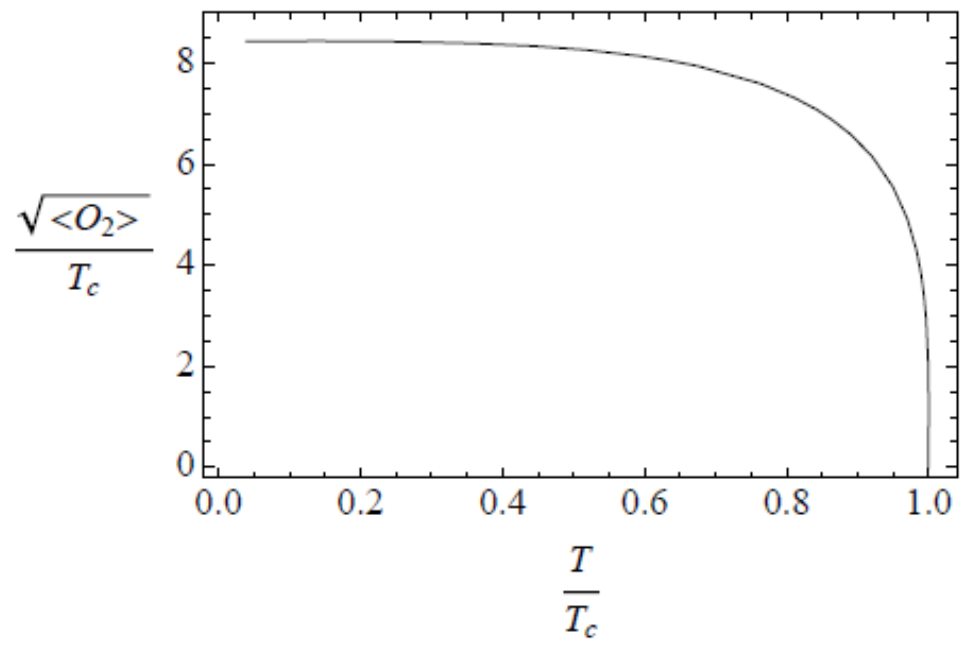

Figura 2.1: Condensado adimensionalizado en función de la temperatura adimensionalizada.

Una solución exacta de las ecuaciones de movimiento es $\psi=0$ y $\phi=\mu-\rho / r$. Parece difícil encontrar otra solución analítica de las ecuaciones de movimiento, pero es inmediato integrarlas numéricamente. Como se ve en la figura 2.1.1 para que dichas soluciones existan, se debe estar a una temperatura lo suficientemente baja.

La figura muestra un condensado con un comportamiento similar al de BCS y al de muchos materiales, donde el condensado va a una constante a temperaturas bajas. Más aun, un ajuste cerca de la temperatura crítica muestra un comportamiento del tipo

$$
\left\langle\mathcal{O}_{2}\right\rangle \approx\left(T-T_{c}\right)^{1 / 2}
$$

El exponente crítico $1 / 2$ se corresponde con el valor universal de campo medio, donde las correcciones por loops estarían suprimidas por ser una teoría a $N$ (rango del grupo de gauge) grande. Más aún, transiciones de fase con rotura de simetrías continuas a temperatura finita son solo posibles en $2+1$ dimensiones si consideramos $N$ grande, donde las fluctuaciones están suprimidas. Estas transiciones van a convertirse en crossovers a $N$ finito.

En resumen, para cierta $T<T_{c}$, aparecen soluciones con condensado que rompen la simetría $U(1)$. Es de esperar que esta rotura de simetría implique superconductividad y es en efecto lo que sucede. Sin embargo dejaremos este análisis recién para el capítulo 4, donde se tratarán en un modelo más general. Ahora pasaremos a explicar el rol de las impurezas en este tipo de superconductores, y cómo alteran las mismas el comportamiento del condensado y la densidad de carga.

\subsection{Un superconductor holográfico sucio}

"Yo no se si podré dominar la morbosa tentación de saber más y más de lo que nunca quise saber."

Bola de Nieve 
En esta sección implementaremos los efectos del ruido en superconductores holográficos mediante la introducción de un potencial químico aleatorio en el borde. Consideramos varias realizaciones del ruido y encontramos que la temperatura crítica $T_{c}$ aumenta. También presentamos evidencia para una forma precisa de renormalización en este sistema. Es decir, cuando el potencial químico está caracterizado por un espectro de potencias $k^{-2 \alpha}$ encontramos que el espectro para el condensado y la densidad de carga están precisa y universalmente gobernados por funciones lineales de $\alpha$.

\subsubsection{Introducción}

El desorden es un paradigma fundamental en física de materia condensada ya que provee un importante paso alejándose de los sistemas limpios y hacia los sistemas más reales [31]. Debido a las dificultades técnicas intrínsecas involucradas, el estudio de la relación entre desorden e interacciones en sistemas cuánticos de muchos cuerpos vio poco progreso desde el punto de vista teórico. Sin embargo, recientemente en el contexto de conductores desordenados, Basko, Aleiner and Altshuler dieron un paso formidable en esta dirección presentado evidencias a favor de una fase delocalizada, basada en un análisis perturbativo en la interacción electrón-electrón a todo orden [32]. Trabajos posteriores (ver [33, 34, 36, 35] y sus referencias) confirmaron y afilaron la idea de la existencia de una transición de fases que separa los límites débil y fuertemente interactuantes en sistemas de electrones.

La correspondencia AdS/CFT provee un marco de trabajo natural para describir sistemas fuertemente acoplados. Es natural entonces estudiar la relación entre desorden e interacciones en este contexto. De hecho, han habido discusiones en este sentido: [37, 38, 39, 40, 41, 42]. En este capítulo sin embargo, seguimos el método más directo de acoplar la teoría a un operador cuya fuente tiene una dependencia espacial explícita. Básicamente traducimos el típico protocolo de acción condmatero [31] al contexto de AdS/CFT.

Una aplicación particularmente importante del desorden es en contexto de superconductores suscios que tienen una rica historia en el contexto de materia condensada desde los tiempos del trabajo pionero de Anderson en 1959 [44]. Por muchos años el teorema de Anderson, que dice que la superconductividad es insensible a perturbaciones que preservan reversión temporal (rotura de pares), proveyó la intuición principal. Críticas al argumento de Anderson surgieron por ejemplo en [46, 47, 45] donde los efectos de localización fuerte fueron considerados. De manera más general, la pregunta sobre el rol de las interacciones, en particular, en particular la interacción de Coulomb no puede darse por entendida. Teniendo en cuenta esta situación, vale la pena considerar otro punto de vista, donde el problema pueda ser atacado en detalle.

La correspondencia AdS/CFT provee un campo de juego perfecto para el estudio de desorden en un superconductor donde la interacción es grande. Esto es precisamente lo que hacemos en esta sección, permitiendo que el potencial químico sea ahora una función aleatoria con cierta dependencia espacial.

Nos concentraremos en dos aspectos. El primero y más intuitivo, es el efecto del desorden en la temperatura crítica. El segundo aspecto es el estudio de cierta universalidad del espectro de potencias del condensado y la densidad de carga como funciones del espectro de potencias de la señal que define el ruido. Es decir, para una dada señal ruidosa aleatoria con espectro $k^{-2 \alpha}$ estudiamos el espectro de potencias del condensado $k^{-2 \Delta(\alpha)}$ y de la densidad de carga $k^{-2 \Gamma(\alpha)}$ y reportamos un comportamiento interesante. Interpretamos este resultado como una 
forma particular de renormalización.

\subsubsection{Superconductor holográfico sucio}

Para construir un superconductor ruidoso $s$-wave en $2+1$ dimensiones empezamos con la acción introducida originalmente en [27, 28] y explicada en la sección anterior. Es decir, Consideramos la dinámica de un escalar y un campo de Maxwell

$$
S=\int d^{4} x \sqrt{-g}\left(-\frac{1}{4} F_{a b} F^{a b}-\left(D_{\mu} \Psi\right)\left(D^{\mu} \Psi\right)^{\dagger}-m^{2} \Psi^{\dagger} \Psi\right) .
$$

en la métrica fija de Schwarzschild-AdS

$$
\begin{aligned}
d s^{2} & =\frac{1}{z^{2}}\left(-f(z) d t^{2}+\frac{d z^{2}}{f(z)}+d x^{2}+d y^{2}\right), \\
f(z) & =1-z^{3}
\end{aligned}
$$

donde pusimos el radio de AdS, $L=1$, y el horizonte en $z_{h}=1$. Estamos usando ahora como coordenada de AdS $z=1 / r$. Consideremos ahora el siguiente Ansatz (consistente) para los campos de materia:

$$
\begin{aligned}
& \Psi(x, z)=\psi(x, z), \quad \psi(x, z) \in \mathbb{R} \\
& A=\phi(x, z) d t
\end{aligned}
$$

Las ecuaciones de movimiento resultantes quedan

$$
\begin{aligned}
& \partial_{z}^{2} \phi+\frac{1}{f} \partial_{x}^{2} \phi-\frac{2 \psi^{2}}{z^{2} f} \phi=0 \\
& \partial_{z}^{2} \psi+\frac{1}{f} \partial_{x}^{2} \psi+\left(\frac{f^{\prime}}{f}-\frac{2}{z}\right) \partial_{z} \psi+\frac{1}{f^{2}}\left(\phi^{2}-\frac{m^{2} f}{z^{2}}\right) \psi=0
\end{aligned}
$$

En lo que sigue, vamos a considerar un escalar con $m^{2}=-2$, que se corresponde con un operador dual de dimensión 2.

Estudiemos ahora el comportamiento asintótico UV de las ecuaciones (2.16), (2.17). Cerca de $z=0$ la solución viene dada por

$$
\begin{aligned}
& \phi(x, z)=\mu(x)+\rho(x) z+\phi^{(2)}(x) z^{2}+o\left(z^{3}\right), \\
& \psi(x, z)=\psi^{(1)}(x) z+\psi^{(2)}(x) z^{2}+o\left(z^{3}\right)
\end{aligned}
$$

donde $\mu(x)$ y $\rho(x)$ corresponden al potencial químico y densidad de carga respectivamente. Las funciones $\psi^{(1)}(x)$ y $\psi^{(2)}(x)$ están identificadas a través de la dualidad con la fuente y el VEV de un operador de dimensión 2. Los términos a orden más altos en esta expansión a $z$ pequeño se pueden escribir en términos de $\mu(x), \rho(x), \psi^{(1)}(x)$ y $\psi^{(2)}(x)$. En el IR, requerir regularidad implica que $A_{t}$ es nulo en el horizonte $z=1$. Entonces debemos considerar una expansión de la forma

$$
\begin{aligned}
& \phi(x, z)=(1-z) \phi_{h}^{(1)}(x)+(1-z)^{2} \phi_{h}^{(2)}(x)+\ldots \\
& \psi(x, z)=\psi_{h}^{(0)}(x)+(1-z) \psi_{h}^{(1)}(x)+(1-z)^{2} \psi_{h}^{(2)}(x)+\ldots,
\end{aligned}
$$


donde los puntos suspensivos representan los términos de orden superior.

Redefiniendo el escalar

$$
\chi(x, z)=\frac{1-z}{z} \psi(x, z),
$$

llegamos a las condiciones de contorno

$$
\begin{aligned}
& \chi(x, 0)=0, \quad \phi(x, 0)=\mu(x), \quad \mathrm{UV} z \rightarrow 0, \\
& \chi(x, 1)=0, \quad \phi(x, 1)=0, \quad \text { IR } z \rightarrow 1 .
\end{aligned}
$$

Esta elección de condiciones de contorno se corresponde con la rotura espontánea de la simetría $U(1)$ con parámetro de orden $\langle\mathcal{O}\rangle \propto \psi^{(2)}(x)$. De ahora en adelante, por lo que resta de este capítulo vamos a usar $\operatorname{los}\langle\ldots\rangle$ asociados a $\mathcal{O}$ exclusivamente para hablar de promedios con respecto a $x$.

\subsubsection{Introduciendo el desorden}

Estamos interesados en resolver el sistema definido poe las ecuaciones (2.16) y (2.17) en presencia de desorden. Vamos a tomar la siguiente forma para el potencial químico

$$
\begin{aligned}
\mu(x) & =\mu_{0}+\epsilon \sum_{k=k_{0}}^{k_{*}} \sqrt{S_{k}} \cos \left(k x+\delta_{k}\right)= \\
& =\mu_{0}+\epsilon \sum_{k=k_{0}}^{k_{*}} \frac{1}{k^{\alpha}} \cos \left(k x+\delta_{k}\right),
\end{aligned}
$$

donde $\delta_{k}$ es una fase aleatoria para cada $k$ y $S_{k}$ es el espectro de potencias. Salvo que se diga lo contrario, vamos a considerar $\alpha=1$. Esto significa que nuestro ruido será continuo pero sin derivadas. Discretizamos el espacio y consideramos condiciones de borde periódicas en la dirección $x$, lo que conduce a considerar $k$ con valores

$$
k_{n}=\frac{2 \pi n}{L} \quad \text { con } \quad 1 \leq n \leq \frac{L}{a}-1
$$

donde $L$ es el largo en la dirección $x$ de nuestro espacio cilíndrico, y a es el espaciado de la red en $x$. Nótese que hay una escala IR $k_{0}$ y una UV $k_{*}$. En el límite de un número grande de modos, la ecuación (2.22) tiende a una función aleatoria distribuida de manera gaussiana; nótese también que el exponente asociado a $1 / k$ determina las propiedades de diferenciabilidad de $\mu(x)$.

Los resultados en esta sección fueron presentados en [25].

Resolvemos el sistema definido por las ecuaciones (2.16) y (2.17) con las condiciones de contorno (2.21) usando una discretización de Rune de segundo orden. La mayoría de las simulaciones fueron hechas de manera independiente en Mathematica y en Python. Las últimas fueron corridas en el University of Michigan Flux cluster. Un resultado típico contiene una red de $100 \times 100$ puntos pero hemos llegado hasta $200 \times 200$ para controlar cuestiones de convergencia y optimización. Usamos un algoritmo de relajación para obtener las soluciones y una medida $\mathcal{L}_{2}$ para la convergencia (definida como la integración de la distancia entre la solución a dos pasos del proceso de relajación distintos), que en la mayoría de los casos llegó a $10^{-16}$. Como fuente del desorden utilizamos $\mu(x)$ dado por $(2.22)$. 
Las escalas involucradas en el problema son: el radio de AdS $R$, la temperatura del agujero negro $z_{h}$, el potencial químico promedio $\mu_{0} \mathrm{y}$ el tamaño del sistema $k_{0}$. Para una realización del ruido caracterizada por la ecuación (2.22) caracterizamos la intensidad del desorden mediante la introducción del parámetro $w$ como $\epsilon=\frac{2}{5} \mu_{0}(w / 10)$, donde $w=0$ corresponde al caso homogéneo y $w=10$ al máximo $\epsilon_{\max }=\frac{2}{5} \mu_{0}$. Elegimos este máximo pidiendo que $\mu(x)$ sea positivo ya que no tenemos una interpretación clara para un ruido que haga las dispersiones más grandes que las medias. Con esta elección de parámetros nos quedamos básicamente con dos escalas que definirán el espacio de fases, la temperatura adimensionalizada $T / \mu_{0}$ y la intensidad del ruido $w$.

\subsubsection{Resultados}

Una manera intuitiva de resumir nuestros resultados viene dada por la figura (2.2) donde registramos el valor adimensionalizado del condensado $\langle\mathcal{O}\rangle / \mu^{2}$ como función del ruido adimensional $w$. Acá $\langle\ldots\rangle$ significa promediar en $x$. Una cuestión clave de esta Figura es el hecho de que la presencia de ruido produce la existencia de condensado para valores del potencial químico para los cuales no habría condensado a $w=0$. La figura (2.2) fue hecha considerando una realización del ruido. Esperamos que los resultados sean estables al considerar varias realizaciones, ya que hemos corroborado que las barras de error son lo suficientemente pequeñas. Este incremento de la superconductividad es uno de nuestros principales resultados.

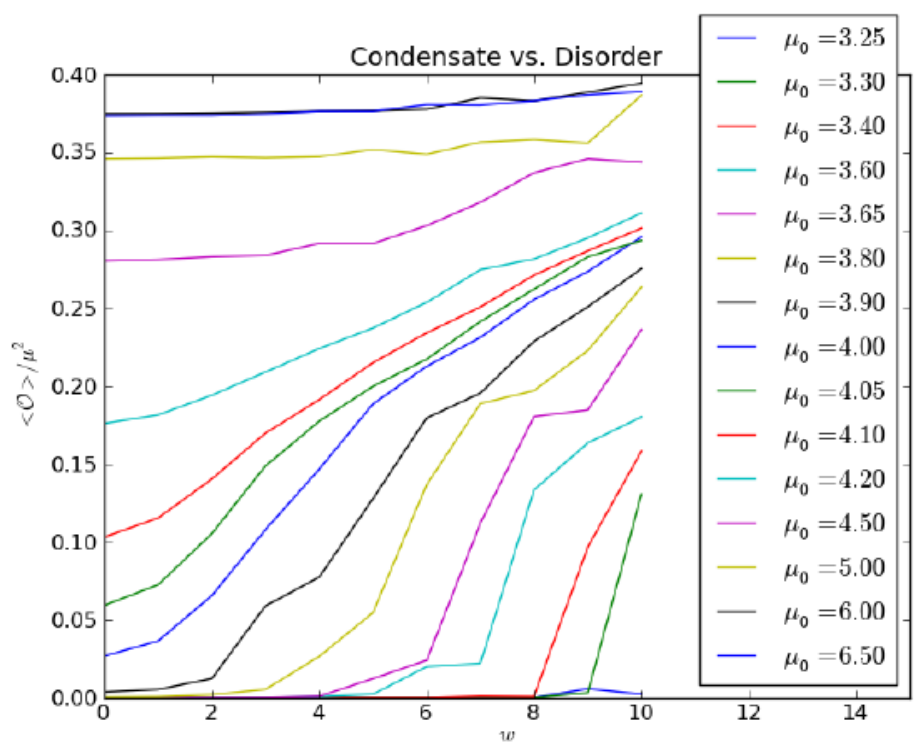

Figura 2.2: Promedio del condensado como función de la amplitud del desorden usando $k_{0}=1$. El valor del condensado crece al crecer la amplitud del desorden, $w$.

En un gráfico separado estudiamos oca más detalle el incremento de $T_{c}$ a medida que incrementamos le ruido $w$. Es decir, elegimos un valor (25\% del máximo) para el cual consideramos que el condensado es claramente diferente de cero y encontramos el valor más pequeño del potencial químico promediado para el cual $w$ lleva a un condensado no nulo. 
Los resaltados aparecen en la figura 2.3 que representa nuestra version del diagrama de fases presentado por ejemplo en [49] para verdaderos superconductores $s$-wave. Compararemos brevemente nuestros resultados con la literatura en las conclusiones.

Notamos que el parámetro $k_{0}$ afecta los resultados numéricamente. Recuérdese que la escala $k_{0}$ está relacionada con la compactificación de la coordenada $x$. Lo que importa al final es la relación con respecto a $L$ el rango de la coordenada $z$ que está fijo a uno. Las simulaciones usadas para generar las figuras 2.2 y 2.3 usaron $k_{0}=1$. Por ejemplo, tomar $k_{0}=2 \pi$, lleva a un incremento más modesto del valor del condensado.

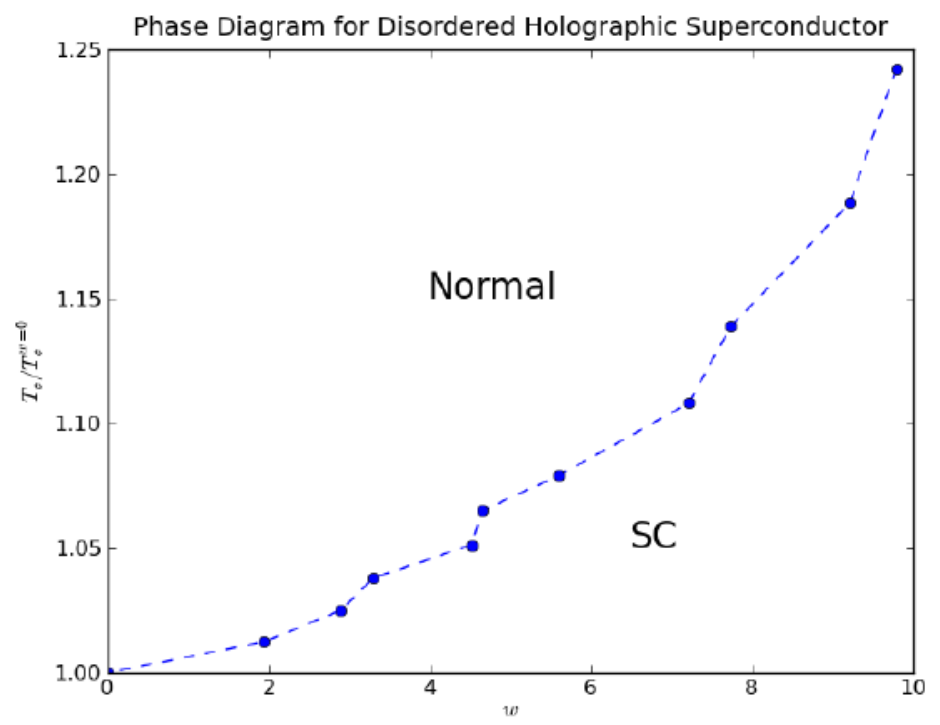

Figura 2.3: Diagrama de fases, dependencia de la temperatura crítica con la amplitud del desorden.

Un resultado bastante genérico es que con potenciales químicos muy discontinuos en el borde, encontramos dependencias en $x$ bastante suaves para el condensado. Un gráfico típico de $\mu(x)$ y su $\mathcal{O}(x)$ correspondiente están representados en la figura (2.4). Por otro lado, lo contrario ocurre para la densidad de carga. Un potencial químico ruidoso se traducirá en una densidad de carga aún más ruidosa. Una forma típica de $\mu(x)$ y su correspondiente $\rho(x)$ aparecen en la figura 2.5 .

Este tipo de suavización/enruidización nos habla de algún tipo de renormalización. Para caracterizar esta renormalización de manera cuantitativa consideramos un ruido del tipo (2.22) pero tomando ahora diferentes valores para $\alpha$. La elección de este parámetro $\alpha$ determina el grado de diferenciabilidad del perfil inicial. Para hacer esto más claro, consideremos el espectro de potencias de $\mu(x)$ que es esencialmente proporcional a $k^{-2 \alpha}$; también consideremos el espectro del condensado $\mathcal{O}(x)$ que aproximaremos como $k^{-2 \Delta(\alpha)}$; similarmente, aproximamos el espectro de potencias de la densidad de carga $\rho(x) \operatorname{como} k^{-2 \Gamma(\alpha)}$. Abajo presentamos el gáfico para $\Delta$ y $\Gamma$ versus $\alpha$ para un amplio rango de valores. Las barras de error fueron calculadas considerando varias realizaciones del mismo ruido.

Colectamos evidencia de que este comportamiento es independiente de la masa del campo escalar, o, en lenguaje de teoría de campos, es independiente de la dimensión conforme del 

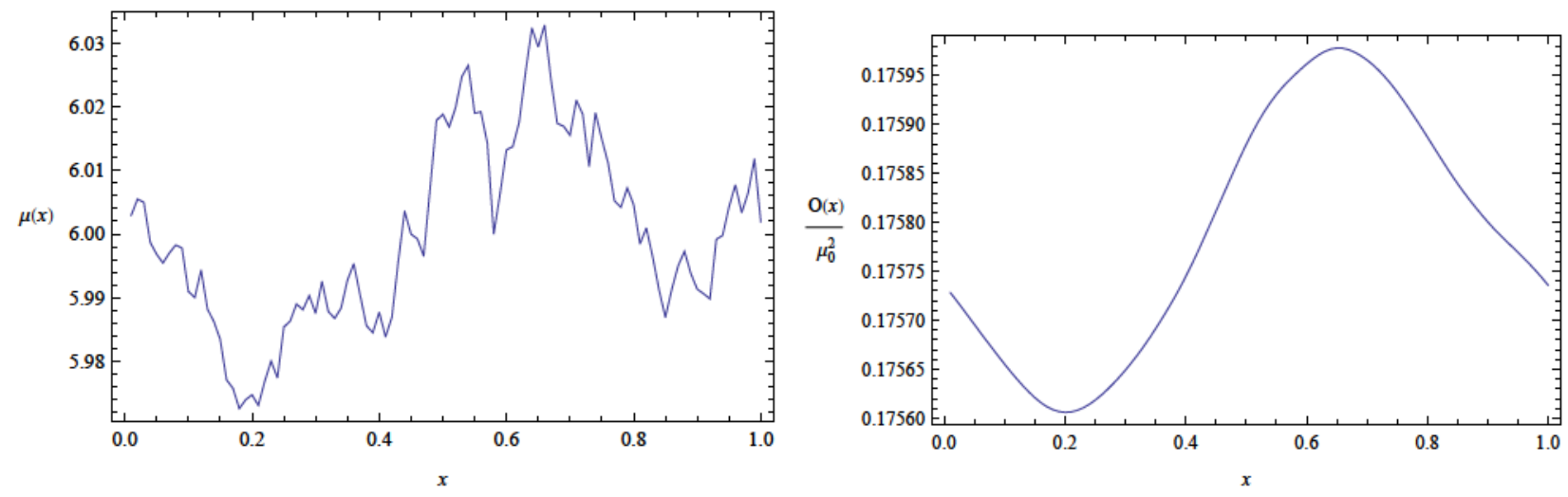

Figura 2.4: Perfil del potencial químico $\mu(x)=6,0+0,1 \sum_{n=1}^{100} \frac{1}{2 \pi n} \cos \left(2 \pi n x+\delta_{n}\right.$ ) (panel izquierdo) y el correspondiente perfil del condensado (panel derecho).
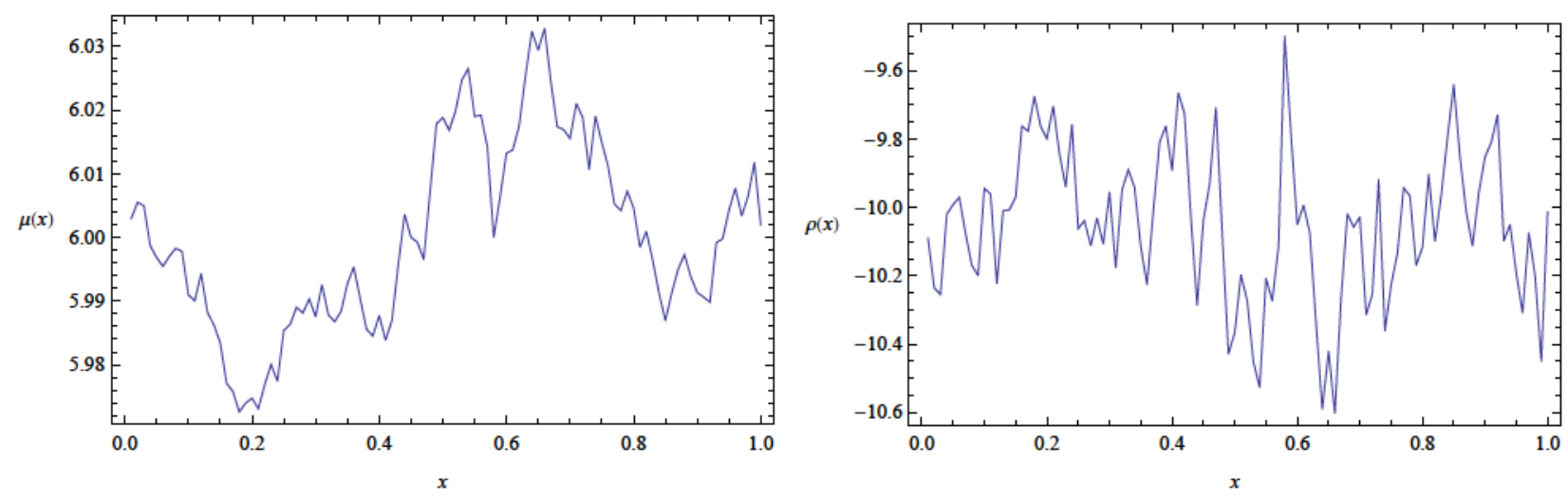

Figura 2.5: Perfil inicial para le potencial químico $\mu(x)=6,0+0,1 \sum_{n=1}^{100} \frac{1}{2 \pi n} \cos \left(2 \pi n x+\delta_{n}\right)$ (panel izquierdo) y el correspondiente perfil para la densidad de carga (panel derecho).
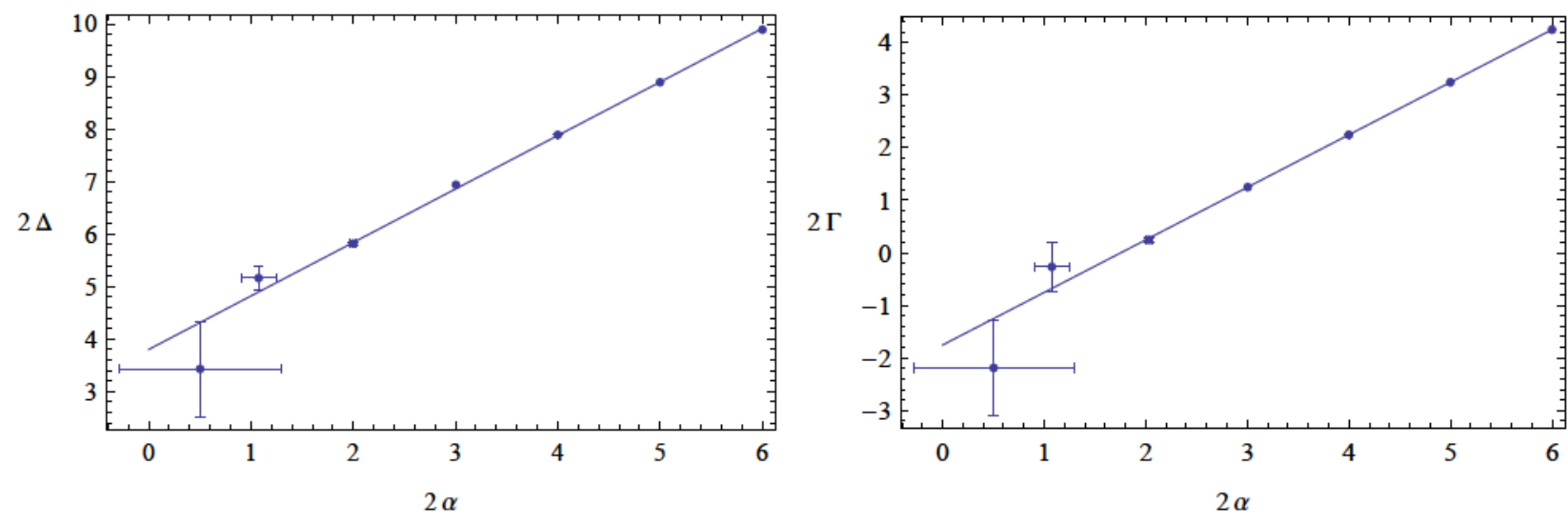

Figura 2.6: Renormalización del desorden: Condensado $\Delta=3,8+1,0 \alpha$ (panel izquierdo) y densidad de carga $\Gamma=-1,75+1,0 \alpha$ (panel derecho). Esta figura fue hecha considerando $k_{0}=2 \pi, \mu_{0}=6$ y $\epsilon=1$. Podemos ver que mientras más plano es el espectro, mayor es el error. Esto puede ser asociado al hecho de que momentos más grandes son más sensibles a la discretización del sistema. 
parámetro de orden. Por ejemplo, confirmamos un comportamiento similar para $m^{2}=0$. Esta universalidad del RG es una de las principales observaciones de nuestro trabajo y su origen parece estar en la naturaleza fuertemente acoplada del problema. La intuición a acoplamiento débil nos dice que $\Delta$ debería ser bien aproximada por la dimensión conforme del parámetro de orden, pero acá corroboramos que no es el caso.

También es importante señalar que este comportamiento no depende de ninguno de los parámetros libres de la teoría, i.e. $k_{0}, \mu_{0}$ o $\epsilon$. Esto significa que podemos rehacer (2.6) para la densidad de carga en la fase normal. Este caso particular es interesante, ya que la teoría se vuelve lineal y podemos separar variables. Siendo ese el caso, podemos recomputar el espectro de potencias usando ahora un simple comando NDSolve de Mathematica. En este caso tenemos $\Gamma=-1,75+1,0 \alpha$, que está de acuerdo con el resultado presentado en la figura (2.6) donde las ecuaciones fueron integradas a través de un método de relajación.

\subsubsection{Conclusiones}

En esta sección reportamos dos interesantes descubrimientos: (i) La temperatura crítica de superconductores holográficos incrementa a medida que se incrementa el nivel de ruido (ii) Es espectro de potencias del condensado y de la densidad de cargas está gobernado por relaciones bastante universales que dependen del espectro de potencias de la señal original.

Comparemos cuidadosamente nuestros resultados con la situación en materia condensada. En la literatura de materia condensada algunos resultados apuntan a una caída de la temperatura crítica $T_{c}$ a medida que el desorden se hace más fuerte [46], [49]. Otros resultados en cambio a apuntan a un incremento de $T_{c}$ [53]. El rol preciso de las interacciones en estos estudios es difícil de determinar. Nuestros resultados claramente apuntan a un incremento de $T_{c}$ pero debemos advertir al lector que el rol de las interacciones en nuestro caso es central y una comparación directa con otros estudios teóricos en materia condensada requeriría una considerable cantidad de trabajo, como el caso de [32] para conductores sucios, esto es, un trabajo que permita sumar las interacciones electrón electrón a todo orden.

Vale la pena señalar que otras discusiones hológrafas, que podrían se consideradas precursoras técnicas de nuestro trabajo en el sentido de que resuelven versiones simplificadas de nuestro sistema, parecen apuntar a un incremento de $T_{c}$. Por ejemplo, [54] consideró una fuente dependiente del tiempo que lleva en cierto rango de frecuencias a un incremento de $T_{c}$, ver sin embargo [55] (también [56]). Potenciales químicos espacialmente modulados considerados, por ejemplo, en [51], [57] (ver también [58]) señalan en cambio una reducción de $T_{c}$.

La universalidad del resultado $k^{-\alpha} \mapsto\left(k^{-\Delta(\alpha)}, k^{-\Gamma(\alpha)}\right)$ para el parámetro de orden y la densidad de carga parecen ser algo propio de las ecuaciones de gravedad. También dijimos que este resultado es independiente de la masa del escalar considerado. Una conclusión inmediata e intrigante es que el operador responsable del RG no es aquel al cual el condensado se acopla. Esta "universalidad" es interesante en el marco de AdS/CFT y merece mayor investigación.

El incremento de $T_{c}$ con el tamaño del ruido en superconductores holográficos merece también mayor atención. Podría ser acaso una predicción de superconductividad holográfica. Sería particularmente interesante considerar otros tipos de superconductores (como por ejemplo, $p$-wave [29]), y llevar un análisis similar en ese caso. Dejamos esto para futuras investigaciones.

En esta sección nos concentramos en el comportamiento del condensado y la densidad 
de carga promediados en $x$. Parece haber una rica estructura en la dependencia en $x$ de dichas cantidades (ver figuras (2.4) y (2.5)). En particular, el condensado parece mostrar potenciales islas de superconductividad. Sería interesante estudiar la aparición de dichas islas y también el efecto de diferentes ruidos en la conductividad (ver [59]). Esperamos atender dichas cuestiones en futuras publicaciones. 


\title{
Capítulo 3
}

\section{Superconductores tipo $\mathrm{p}$}

\author{
"The guide is definitive. Reality is frequently inaccurate."
}

Douglas Adams

En este capítulo se analizará la deformación de la geometría del espacio-tiempo dual a un superconductor tipo $p$ en $2+1$ dimensiones [29] (véase [66, 67] para un tratamiento similar en $3+1$ dimensiones). En el camino se reproducirá la deformación del dual gravitatorio al superconductor tipo $p+i p$ estudiado en [26]. También se usará la prescripción dada en [62, $65,68]$ para calcular la entropía de entrelazamiento desde el punto de vista holográfico para ambos duales gravitatorios. Cálculos similares de la entropía de entrelazamiento en pueden encontrarse en [69, 70, 71, 72]. Este capítulo fue presentado en la tesis [61] y originalmente en [60].

La superconductividad tipo $p$ es una fase de la materia que se produce cuando los electrones con momento angular relativo $j=1$ condensan formando pares de Cooper. En otras palabras, existe un vector cargado ante una simetría $U(1)$ que condensa. Este tipo de superconductores se originan a partir de electrones fuertemente correlacionados y por lo tanto la utilización de la teoría de Bardeen, Cooper y Schrieffer (BCS) no es la manera correcta de describir su dinámica microscópica. Este fenómeno es un desafío para la física teórica que, debido a la propiedad fundamental de la conjetura de describir teorías de campos fuertemente acopladas, puede ser estudiado a partir de su dual gravitatorio. Se introducirán ahora los ingredientes mínimos necesarios para reproducir la dinámica del superconductor. Este tipo de enfoque nos permite reproducir las propiedades del sistema de materia condensada sin explicar su origen microscópico.

En los trabajos $[73,74,75,76,77,78]$ fueron estudiados superconductores tipo $p$ a partir de una teoría de cuerdas dual. Los mínimos ingredientes necesarios en la teoría gravitatoria para tener temperatura finita, potencial químico y ruptura espontánea de simetría (SSB) son: una geometría de agujero negro y un campo de gauge no Abeliano [79]. Las soluciones a ser consideradas son geometrías asintóticamente AdS con un campo de gauge $S U(2)$. La SSB se realiza mediante una condición asintótica no trivial (pelo) sobre este campo de gauge. El potencial químico y la SSB provienen de prender dos direcciones independientes dentro del grupo de gauge no Abeliano. La ruptura espontánea de simetría ocurre en el lado gravitatorio a través de la formación de un condensado fuera del horizonte.

La entropía de entrelazamiento (EE) entre un subsistema $\mathcal{A}$ y su complemento $\mathcal{B}$ está 
definida por la entropía de von Neumann

$$
\mathcal{S}_{\mathcal{A}}=-\operatorname{Tr}_{\mathcal{A}}\left(\rho_{\mathcal{A}} \ln \rho_{\mathcal{A}}\right)
$$

Aquí $\rho_{\mathcal{A}}=\operatorname{Tr}_{\mathcal{B}}(\rho)$ es la matriz densidad obtenida de tomar la traza sobre los grados de libertad del subsistema $\mathcal{B}$ en la matrix densidad del sistema completo $\rho$. Inogenuamente $S_{\mathcal{A}}$ mide la cantidad de información que está oculta dentro de $\mathcal{B}$ cuando subdividimos el sistema. Desde el punto de vista de la teoría de gravedad la EE fue conjeturada [62] proporcional al valor del área mínima para una superficie, $\gamma_{\mathcal{A}}$, en el bul cuyo borde en el borde de AdS coincide con el borde de la región $\mathcal{A}$ (véase [68])

$$
\mathcal{S}_{\mathcal{A}}=\frac{2 \pi \operatorname{Area}\left(\gamma_{\mathcal{A}}\right)}{\kappa^{2}}
$$

Acá $\kappa$ es la constante gravitatoria. Nótese que la entropía térmica estándar se obtiene como un caso particular de la EE cuando la región $\mathcal{A}$ es el sistema entero. En [80] los autores demostraron cómo esta técnica holográfica para calcular la EE arroja el resultado correcto al estudiarse el caso particular de una superficie esférica y a temperatura cero en teorías de campos conformes. Al final de este capítulo se calculará la EE para una geometría con forma de banda en los duales gravitatorios correspondientes a los superconductores tipo $p$ y $p+i p$.

\subsection{Superconductores tipo $p$ y $p+i p$}

Como ya ha sido mencionado, el dual gravitatorio a un superconductor tipo $p$ se modela con una teoría de Einstein-Yang-Mills (EYM). En [29, 81] fue estudiada dicha teoría de gravedad en $3+1$ dimensiones y en su límite de prueba, es decir, despreciando la deformación en la geometría debida al campo de gauge. Además, estos autores mostraron que la teoría dual al superconductor tipo $p+i p$ estudiada en [26] es inestable ante pequeñas fluctuaciones siendo la configuración estable aquella para el superconductor tipo $p$. En esta sección se tendrán en cuenta las deformaciones de la geometría $3+1$ dimensional dual al superconductor $p$ producidas por el campo de gauge no Abeliano y se compararán los resultados con los obtenidos para el caso del $p+i p$.

Se trabajará con un grupo de gauge $S U(2)$. En el caso del $p+i p$, se propondrá un campo de gauge tal que rompa el subgrupo $U(1)$ del $S U(2)$ y la simetría rotacional $S O(3)$ del espacio pero que deje invariante un subgrupo diagonal de ellos. Por otro lado, en el dual al superconductor tipo $p$, el campo de gauge romperá ambas simetrías $U(1)$ por completo. La solución gravitatoria que describe la dinámica de acoplamiento fuerte en ambos tipos de superconductores se describe de la siguiente manera: se forma una capa superconductora cargada fuera del horizonte producida por la competencia entre la repulsión eléctrica (con el agujero negro cargado) y el potencial gravitatorio de la geometría asintóticamente AdS. A temperaturas suficientemente altas no se produce pelo fuera del agujero negro y la solución es AdS-Reissner-Nordström (AdSRN). Por debajo de una temperatura crítica $T_{c}$ se genera un campo de gauge no trivial con potencial químico no nulo sobre el borde de la geometría y aparece un condensado sin necesidad de tener una fuente para el mismo, lo cual origina un rompimiento espontáneo de la simetría de gauge $S U(2)$. 


\subsubsection{Superconductor tipo $p$ en $2+1$ dimensiones}

\section{Solución}

Comencemos a partir de una teoría de Yang-Mills en $3+1$ dimensiones y con grupo de gauge $S U(2)$ en un espacio AdS (ver [82]), su densidad Lagrangiana es

$$
\kappa_{(4)}^{2} \mathcal{L}=R-2 \Lambda-\frac{1}{4} \operatorname{Tr}\left(F_{\mu \nu} F^{\mu \nu}\right)
$$

en donde $\Lambda=-\frac{3}{\hat{R}^{2}}, \kappa_{(4)}$ es la constante gravitacional en cuatro dimensiones y el $F_{\mu \nu}$ para el campo de gauge es

$$
F_{\mu \nu}^{a}=\partial_{\mu} A_{\nu}^{a}-\partial_{\nu} A_{\mu}^{a}+g_{Y M} \epsilon^{a b c} A_{\mu}^{b} A_{\nu}^{c}
$$

con $g_{Y M}=\frac{\hat{g}_{Y M}}{\kappa_{(4)}}$ denotando al parámetro que mide la deformación de la geometría y $\hat{g}_{Y M}$ el acoplamiento de Yang-Mills usual. Se usarán letras latinas como índice sobre el grupo $S U(2)$ y letras griegas para denotar coordenadas del espacio-tiempo. Escaleando el campo de gauge como $\tilde{A}=\frac{A}{g_{Y M}}$ se puede ver que el límite de $g_{Y M}$ grande corresponde al límite de prueba del campo de gauge. Aproximadamente uno puede pensar que $\frac{1}{\hat{g}_{Y M}^{2}}$ cuenta el número de grados de libertad cargados ante el $S U(2)$ de la teoría dual. Además, $\frac{1}{\kappa_{(4)}^{2}}$ cuenta el número total de grados de libertad. Considerar la deformación producida por el campo de gauge significa que el número de grados de libertad de los estados cargados es del mismo orden que el número de grados de libertad del sistema.

Las ecuaciones de movimiento obtenidas a partir de minimizar la acción son

$$
\begin{aligned}
G_{\mu \nu}=R_{\mu \nu}-\frac{1}{2} g_{\mu \nu} R & =\frac{3}{R^{2}} g_{\mu \nu}+\frac{1}{2} \operatorname{Tr}\left[F_{\mu \gamma} F_{\nu}^{\gamma}\right]-\frac{g_{\mu \nu}}{8} \operatorname{Tr}\left[F_{\gamma \rho} F^{\gamma \rho}\right] \\
D_{\mu} F^{\mu \nu} & =0
\end{aligned}
$$

y con el objetivo de resolverlas se propondrá la siguiente solución [66, 83]

$$
d s^{2}=-M(r) \sigma(r)^{2} d t^{2}+\frac{1}{M(r)} d r^{2}+r^{2} h(r)^{2} d x^{2}+r^{2} h(r)^{-2} d y^{2}
$$

para la geometría de fondo, y

$$
A=\phi(r) \tau^{3} d t+\omega(r) \tau^{1} d x
$$

para el campo de gauge. Aquí se ha usado la notación matricial $A=A_{\mu}^{a} \tau^{a} d x^{\mu} \operatorname{con} \tau^{a}=\frac{\sigma^{a}}{2 i}$ y $\sigma^{a}$ las matrices de Pauli usuales, los generadores de $S U(2)$ satisfacen $\left[\tau^{a}, \tau^{b}\right]=\epsilon^{a b c} \tau^{c}$. Un solución que satisfaga que $\omega \neq 0$ en el campo de gauge (??) romperá la simetría $U(1)$ asociada con rotaciones alrededor de $\tau^{3}$ (usualmente denominada $U(1)_{3}$ ). Además si $h \neq 0$ se romperá la simetría $U(1)_{x y}$ asociada a rotaciones en el plano $x y$. A temperaturas suficientemente altas se espera que no haya pelo fuera del agujero negro y la solución con condensado nulo es 
AdSRN con

$$
\begin{aligned}
\omega(r) & =0 \\
h(r) & =1 \\
\sigma(r) & =1 \\
\phi(r) & =\mu\left(1-\frac{r_{h}}{r}\right) \\
M(r) & =r^{2}+\frac{\mu^{2} r_{h}^{2}}{r^{2}}-\left(\frac{\mu^{2}}{8}+r_{h}^{2}\right) \frac{r_{h}}{r} .
\end{aligned}
$$

Reemplazando la solución propuesta en las ecuaciones de movimiento para la acción de EYM se obtienen 5 ecuaciones, tres de ellas son ecuaciones diferenciales de segundo orden y las dos restantes son ligaduras de primer orden

$$
\begin{aligned}
M^{\prime} & =\frac{3 r}{\hat{R}^{2}}-\frac{1}{8 \sigma^{2}}\left(\frac{g_{Y M}^{2} \phi^{2} \omega^{2}}{r h^{2} M}+r \phi^{\prime 2}\right)-M\left(\frac{1}{r}+\frac{r h^{\prime 2}}{h^{2}}+\frac{\omega^{\prime 2}}{8 r h^{2}}\right) \\
\sigma^{\prime} & =\frac{\sigma}{h^{2}}\left(r h^{\prime 2}+\frac{\omega^{\prime 2}}{8 r}\right)+\frac{g_{Y M}^{2} \phi^{2} \omega^{2}}{8 r M^{2} h^{2} \sigma} \\
h^{\prime \prime} & =\frac{1}{8 r^{2} h}\left(-\omega^{\prime 2}+\frac{g_{Y M}^{2} \phi^{2} \omega^{2}}{M^{2} \sigma^{2}}\right)-h^{\prime}\left(\frac{2}{r}-\frac{h^{\prime}}{h}+\frac{M^{\prime}}{M}+\frac{\sigma^{\prime}}{\sigma}\right) ; \\
\omega^{\prime \prime} & =-\frac{g_{Y M}^{2} \phi^{2} \omega}{M^{2} \sigma^{2}}+\omega^{\prime}\left(\frac{2 h^{\prime}}{h}-\frac{M^{\prime}}{M}-\frac{\sigma^{\prime}}{\sigma}\right) ; \\
\phi^{\prime \prime} & =\frac{g_{Y M}^{2} \phi \omega^{2}}{r^{2} h^{2} M}-\phi^{\prime}\left(\frac{2}{r}-\frac{\sigma^{\prime}}{\sigma}\right) .
\end{aligned}
$$

Este sistema de ecuaciones goza de 4 simetrías de escala que serán útiles cuando intentemos resolverlas numéricamente, ellas son

1. $\sigma \rightarrow \lambda \sigma, \quad \phi \rightarrow \lambda \phi$

2. $\omega \rightarrow \lambda \omega, \quad h \rightarrow \lambda h$

3. $M \rightarrow \lambda^{-2} M, \quad \sigma \rightarrow \lambda \sigma, \quad g_{Y M} \rightarrow \lambda^{-1} g_{Y M}, \quad \hat{R} \rightarrow \lambda \hat{R}$

4. $M \rightarrow \lambda^{2} M, \quad r \rightarrow \lambda r, \quad \phi \rightarrow \lambda \phi, \quad \omega \rightarrow \lambda \omega$

Usando estas simetrías de escala podemos elegir $\hat{R}=r_{h}=1$ y fijar el valor en el borde de las funciones de la métrica $\sigma(\infty)=h(\infty)=1$. La geometría y el campo de gauge deben ser regulares en el horizonte, esto motiva la siguiente expansión en el IR ( $r$ pequeño)

$$
\begin{aligned}
M & =M_{1}\left(r-r_{h}\right)+M_{2}\left(r-r_{h}\right)^{2}+\ldots \\
h & =h_{0}+h_{2}\left(r-r_{h}\right)^{2}+\ldots \\
\sigma & =\sigma_{0}+\sigma_{1}\left(r-r_{h}\right)+\sigma_{2}\left(r-r_{h}\right)^{2}+\ldots \\
\omega & =\omega_{0}+\omega_{2}\left(r-r_{h}\right)^{2}+\omega_{3}\left(r-r_{h}\right)^{3}+\ldots \\
\phi & =\phi_{1}\left(r-r_{h}\right)+\phi_{2}\left(r-r_{h}\right)^{2}+\ldots
\end{aligned}
$$


Por otro lado, en el UV ( $r$ grande) el desarrollo deseado es

$$
\begin{aligned}
M & =r^{2}+\frac{M_{1}^{b}}{r}+\frac{\left(\omega_{1}^{b}\right)^{2}+\rho^{2}}{8 r^{2}}+\ldots \\
h & =1+\frac{h_{3}^{b}}{r^{3}}-\frac{\left(\omega_{1}^{b}\right)^{2}}{32 r^{4}}+\ldots \\
\sigma & =1-\frac{\left(\omega_{1}^{b}\right)^{2}}{32 r^{4}}+\ldots \\
\omega & =\omega_{0}^{b}+\frac{\omega_{1}^{b}}{r}-\frac{g_{Y M}^{2} \mu^{2} \omega_{1}^{b}}{6 r^{3}}+\ldots \\
\phi & =\mu+\frac{\rho}{r}+\frac{g_{Y M}^{2} \mu^{2} \omega_{1}^{b}}{12 r^{4}}+\ldots
\end{aligned}
$$

Para dar cuenta de la SSB, se buscan soluciones en donde la componente no normalizable se anule $\omega_{0}^{b}=0$. El diccionario estándar de AdS/CFT nos permite interpretar al valor de borde y al sub-leading (segundo termino más relevante en la expansión) de $\phi$ como el potencial químico $\mu$ y la densidad de carga $\rho$ de la teoría de campos dual [84]. Además, el coeficiente sub-leading $M_{1}^{b}$ en la expansión en el borde de $g_{t t}$ coincide con la acción Euclídea regularizada evaluada en la solución de las ecuaciones de movimiento. El coeficiente normalizable en $\omega$ es dual al valor de expectación de vacío de la corriente $\left\langle J_{x}^{1}\right\rangle \propto \omega_{1}^{b}$ y hace las veces de parámetro de orden para el sistema.

Las soluciones de las ecuaciones (3.10) dependen de cuatro coeficientes IR $\phi_{1}, \omega_{0}, h_{0}, \sigma_{0}$ y del parámetro de deformación $g_{Y M}$. Todos los otros coeficientes en (3.11) pueden ser escritos en términos de estos. Se procederá a integrar las ecuaciones de movimiento numéricamente desde el horizonte hacia el borde usando un método de shooting con el objetivo de tener el comportamiento asintótico deseado. Se exploró el rango $g_{Y M} \in[0,85,24]$ y se observó que el comportamiento de las funciones no cambia cualitativamente al variar $g_{Y M}$. En las figuras 3.1 y 3.2 se muestran las soluciones de (3.10) con condiciones de borde (3.12). Se ha usado el potencial químico $\mu$ para adimensionalizar. Esto significa que se esta trabajando en el conjunto gran canónico.

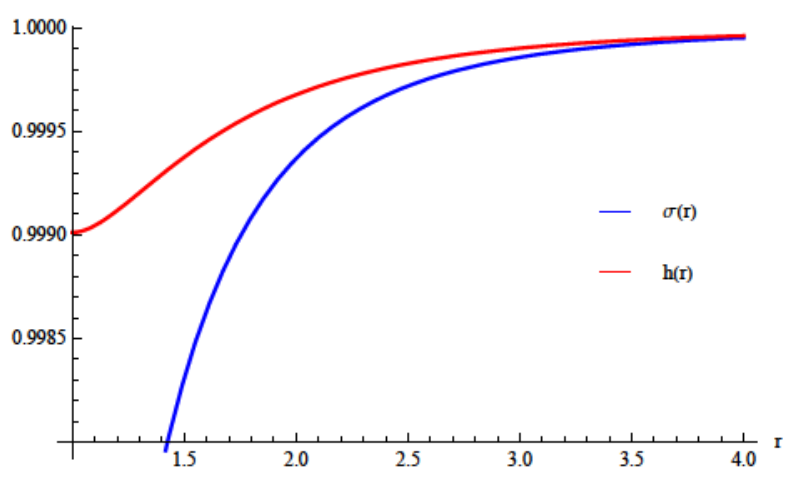

Figura 3.1: Las funciones adimensionales de la métrica $\sigma(r)$ y $h(r)$ para $g_{Y M}=2$ y $T=$ $0,2312 \mu$.

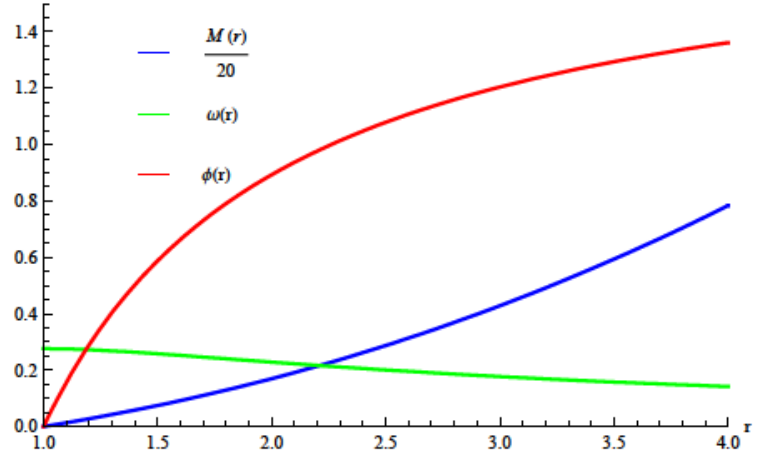

Figura 3.2: Las funciones adimensionales de la métrica $M(r)$ y las funciones del campo de gauge $\omega(r)$ y $\phi(r)$ para $g_{Y M}=2, T=$ $0,2312 \mu$. 


\section{Termodinámica}

En esta sección se calcularán las cantidades termodinámicas asociadas con las soluciones. A partir del estudio de la función potencial en el conjunto ${ }^{1}$ gran canónico se observará una transición de fase de segundo orden entre una fase superconductora y una normal.

La temperatura de la teoría de campos dual está dada por la temperatura de Hawking del agujero negro

$$
T=\frac{M_{1} \sigma_{0}}{2 \pi}=\frac{1}{16 \pi}\left(24 \sigma_{0}^{2}-\phi_{1}^{2}\right) r_{h}
$$

en donde la segunda igualdad proviene de la consistencia de la expansión en serie (3.11) que relaciona el coeficiente $M_{1}$ con $\sigma_{0}$ y $\phi_{1}$. El área del horizonte, $A_{h}$, conduce a la entropía

$$
S=\frac{2 \pi}{\kappa_{(4)}^{2}} A_{h}=\frac{2 \pi^{2} V T^{2}}{\kappa_{(4)}^{2}} \frac{12^{2}}{\left(24 \sigma_{0}^{2}-\phi_{1}^{2}\right)^{2}}
$$

en donde $V=\int d x d y$. En la figura 3.3 se muestra el parámetro de orden $\omega_{1}^{b}$ (es decir, el valor de expectación de la corriente $\left.\left\langle J_{x}^{1}\right\rangle\right)$ como función de la temperatura. Nótese que a $T=T_{c}$ el condensado se anula mostrando la desaparición de el estado superconductor para $T>T_{c}$. A partir de los resultados numéricos se encuentra que cerca de la temperatura crítica $T_{c}$ la corriente satisface $\left\langle J_{1}^{x}\right\rangle \propto\left(1-\frac{T}{T_{c}}\right)^{1 / 2}$ y por lo tanto el valor del exponente crítico es $1 / 2$. La figura 3.4 muestra el comportamiento de la entropía de Bekenstein-Hawking (3.14) como función de la temperatura para nuestra solución y la del agujero negro AdSRN.

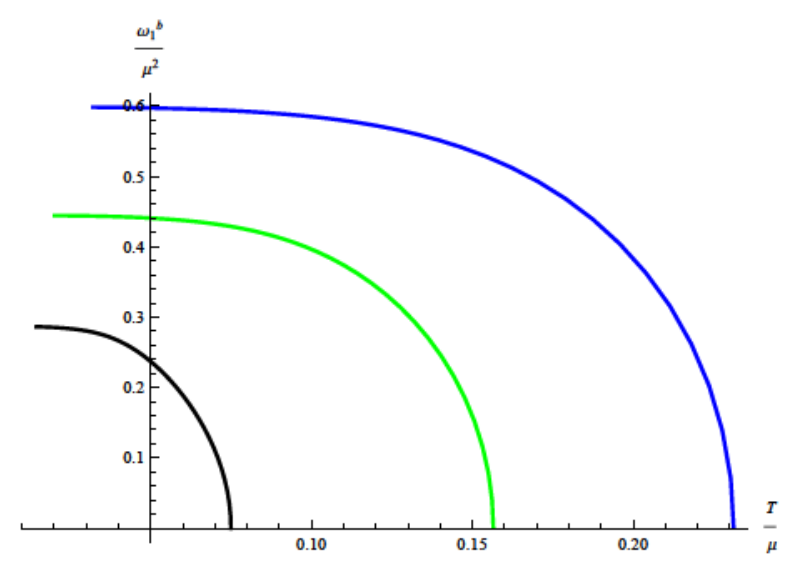

Figura 3.3: La figura muestra el comportamiento del coeficiente normalizable de la función $\omega$, el cual es proporcional al condensado $\left\langle J_{x}^{1}\right\rangle$. Las líneas negra, verde y azul refieren a soluciones con $g_{Y M}=1,1,5,2$ y $T_{c}=0,0749,0,1565,0,2312$ respectivamente. Nótese que el condensado se anula para $T>T_{c}$.

La correspondencia AdS/CFT identifica la acción Euclídea on-shell de la teoría de gravedad $S_{E}$ multiplicada por la temperatura $T$ como la función potencial $\Omega$ del sistema en el conjunto gran canónico. Para calcularla, se hará una continuación a signatura Euclídea y se

\footnotetext{
${ }^{1}$ Para pasar del conjunto canónico ( $\mu$ fijo) con energía libre $\Omega$, al conjunto gran canónico ( $\rho$ fijo) con energía libre $F$, se debe agregar un termino de borde a la acción Euclídea. Esto cambia el problema variacional e implica la relación de Gibbs $F=\Omega+\mu \rho$.
} 


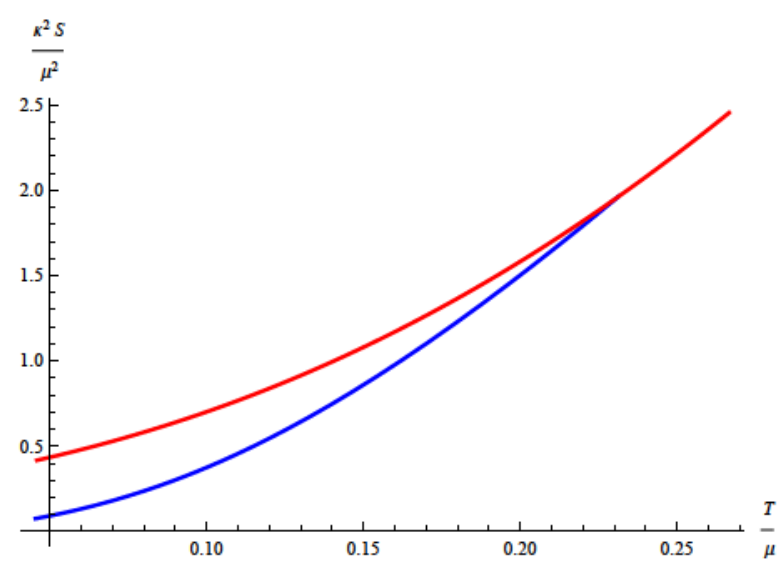

Figura 3.4: Entropía como función de la temperatura. La línea azul es para la fase superconductora con $g_{Y M}=2$ y la línea roja refiere a la fase normal (geometría AdSRN). Hay una transición de fase de segundo orden para $T=T_{c}=0,2312$.

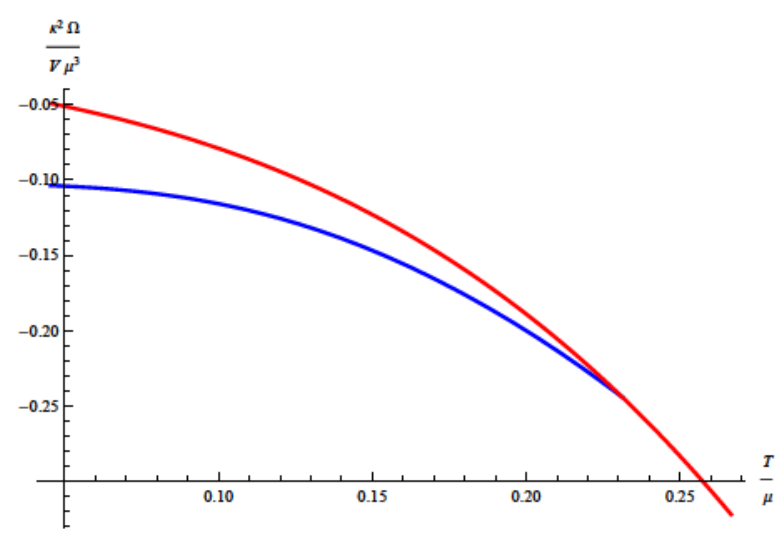

Figura 3.5: Función potencial $\Omega$ calculada a partir de (3.22) como función de la temperatura $T$ para $g_{Y M}=2$. La línea roja es el potencial para la solución de RN y la azul para la fase superconductora.

compactificará el tiempo con periodo $\frac{1}{T}$ para evitar singularidades. La acción on-shell tiene un factor $\frac{1}{T}$ proveniente de la integración en la coordenada temporal, escribiendo $S_{\text {on-shell }}=\frac{\tilde{S}_{\text {bulk }}}{T}$ se tiene

$$
\tilde{S}_{\text {bulk }}=-\int d x d y d r \sqrt{-g} \mathcal{L}
$$

en donde la densidad Lagrangiana está escrita en (3.3). La componente yy del tensor energíamomento es proporcional a la métrica y por lo tanto las ecuaciones de Einstein (3.5) implican que

Luego, tenemos

$$
G_{y y}=\frac{r^{2}}{2 h^{2}}\left(\kappa_{(4)}^{2} \mathcal{L}-R\right)
$$

$$
G_{\mu}^{\mu}=-R=G_{r}^{r}+G_{t}^{t}+G_{x}^{x}+\frac{1}{2}\left(\kappa_{(4)}^{2} \mathcal{L}-R\right)
$$

a partir de lo que se obtiene

$$
\mathcal{L}=\frac{2}{r^{2} \sigma \kappa_{(4)}^{2}}\left[\frac{r^{3} M \sigma}{h}\left(\frac{h}{r}\right)^{\prime}\right]^{\prime}
$$

en donde ' denota derivada con respecto a la coordenada holográfica $r$. Luego, la contribución a la acción on-shell (3.15) proveniente del volumen del espacio-tiempo se puede escribir como

$$
\tilde{S}_{b u l k}=-\int d x d y d r \sqrt{-g} \mathcal{L}=-\frac{2 V}{\kappa_{(4)}^{2}}\left[\frac{r^{3} M \sigma}{h}\left(\frac{h}{r}\right)^{\prime}\right]_{r=r_{\infty}}
$$

en donde $r_{\infty}$ es el borde del espacio. Como es usual, para tener un problema variacional bien definido cuando se imponen condiciones de borde Dirichlet en la métrica necesitamos agregar a la acción el término de Gibbons-Hawking

$$
\tilde{S}_{G H}=-\frac{1}{\kappa_{(4)}^{2}} \int d x d y \sqrt{-g_{\infty}} \nabla_{\mu} n^{\mu}=-\frac{V}{\kappa_{(4)}^{2}} r^{2} \sigma\left[\frac{M^{\prime}}{2}+M\left(\frac{\sigma^{\prime}}{\sigma}+\frac{2}{r}\right)\right]_{r=r_{\infty}}
$$


siendo $n^{\mu} d x_{\mu}=\sqrt{M} d r$ el vector unitario normal al borde y $g_{\infty}$ el determinante de la métrica inducida sobre el borde. Precisamente en $r=r_{\infty}$ (3.20) diverge y por lo tanto debe ser regularizada mediante la suma de contra-términos de borde

$$
\tilde{S}_{c t}=\frac{1}{\kappa_{(4)}^{2}} \int d x d y \sqrt{-g_{\infty}}=\frac{V}{\kappa_{(4)}^{2}}\left[r^{2} \sqrt{M} \sigma\right]_{r=r_{\infty}}
$$

Finalmente, el potencial termodinámico dual $\Omega$ resulta

$$
\begin{aligned}
\Omega & =\lim _{r_{\infty} \rightarrow \infty} \tilde{S}_{\text {on-shell }} \\
& =\lim _{r_{\infty} \rightarrow \infty}\left(\tilde{S}_{\text {bulk }}+\tilde{S}_{G H}+\tilde{S}_{c t}\right)
\end{aligned}
$$

Luego de regularizar la acción el potencial $\Omega$ coincide con el valor sub-leading de la componente $g_{t t}$ de la métrica de fondo, es decir, $\Omega=M_{1}^{b}$ [28]. Se ha verificado la solución numérica calculando $\Omega$ de ambas maneras y se encontró una concordancia excelente. En la figura 3.5 se muestra el potencial (3.22) como función de la temperatura. Como se mencionó anteriormente se produce una transición de fase de segundo orden en $T=T_{c}$ : el gran potencial y la entropía son continuas pero $S$ no es diferenciable. Por debajo de $T_{c}$ el sistema se encuentra en la fase superconductora, al incrementar la temperatura por sobre $T_{c}$ la geometría de AdSRN domina la energía libre, lo que modela la transición de la fase superconductora a la normal.

\subsubsection{Superconductores tipo $p+i p$}

En esta sección se recopilarán los resultados de [26] y se compararán con los resultados obtenidos en la sección anterior. Se encontró que a $T=T_{c}$ el sistema tiene una transición de fase de segundo orden y que en todo el rango de temperatura el gran potencial del superconductor tipo $p$ es menor que el del $p+i p$. Esto implica que la fase estable del sistema es la tipo $p$, lo cual concuerda con el análisis de estabilidad desarrollado en [29].

\section{Solución}

La geometría y el campo de gauge para modelar la solución son

$$
\begin{aligned}
d s^{2} & =-M(r) d t^{2}+r^{2} h(r)^{2}\left(d x^{2}+d y^{2}\right)+\frac{d r^{2}}{M(r)} \\
A & =\phi(r) \tau^{3} d t+\omega(r)\left(\tau^{1} d x+\tau^{2} d y\right) .
\end{aligned}
$$

Aquí hay un diferencia importante con el superconductor tipo $p$ de la sección previa. Ahora el campo de gauge rompe el grupo $U(1)_{3} \times U(1)_{x y}$ dejando invariante una combinación diagonal. En la sección anterior el campo de gauge rompía por completo el grupo $U(1)_{3} \times U(1)_{x y}$. Esto nos permite proponer una métrica totalmente simétrica en el plano $x y$.

Las 5 ecuaciones de movimiento obtenidas se dividen en 4 ecuaciones diferenciales de segundo orden y una ligadura de primer orden proveniente de la componente $r r$ de la ecua- 
ciones de Einstein

$$
\begin{aligned}
h^{\prime \prime} & =-\frac{h}{2}\left[\frac{1}{r^{2}}-\frac{3}{\hat{R}^{2} M}+\frac{M^{\prime}}{r M}+\frac{\phi^{\prime 2}}{8 M}+\frac{\omega^{\prime 2}}{4 r^{2} h^{2}}\right]-\frac{h^{\prime}}{2}\left[\frac{6}{r}+\frac{h^{\prime}}{h}+\frac{M^{\prime}}{M}\right]-\frac{g_{Y M}^{2} \omega^{2}}{8 r^{2} h M}\left[\frac{\phi^{2}}{M}+\frac{\omega^{2}}{2 r^{2} h^{2}}\right] \\
M^{\prime \prime} & =\frac{3}{\hat{R}^{2}}+\frac{M}{r}\left[-\frac{M^{\prime}}{M}+\frac{1}{r}+\frac{\omega^{\prime 2}}{4 r h^{2}}\right]-\frac{h^{\prime}}{h}\left[M^{\prime}-\frac{h^{\prime}}{h}-\frac{2}{r}\right]+\frac{3}{8} \phi^{\prime 2}+\frac{g_{Y M}^{2} \omega^{2}}{4 r^{2} h^{2}}\left[\frac{\phi^{2}}{M}+\frac{3 \omega^{2}}{2 r^{2} h^{2}}\right] \\
\omega^{\prime \prime} & =\frac{g_{Y M}^{2} \omega}{M}\left[\frac{\omega^{2}}{r^{2} h^{2}}-\frac{\phi^{2}}{M}\right]-\frac{M^{\prime} \omega^{\prime}}{M} \\
\phi^{\prime \prime} & =\frac{2 g_{Y M}^{2} \phi \omega^{2}}{r^{2} h^{2} M}-2 \phi^{\prime}\left[\frac{1}{r}+\frac{h^{\prime}}{h}\right] \\
0 & =-\frac{3}{\hat{R}^{2}}+\frac{M}{r^{2}}\left[1-\frac{\omega^{\prime 2}}{4 h^{2}}+\frac{M^{\prime}}{M} r\right]+\frac{h^{\prime}}{h}\left[M\left(\frac{2}{r}+\frac{h^{\prime}}{h}\right)+M^{\prime}\right]+\frac{1}{8} \phi^{\prime 2} .
\end{aligned}
$$

Las ecuaciones tienen tres simetrías de escala que nos ayudarán a resolver el sistema numéricamente. Estas son

1. $\omega \rightarrow \lambda \omega, \quad h \rightarrow \lambda h$,

2. $M \rightarrow \lambda^{-2} M, \quad \phi \rightarrow \frac{\phi}{\lambda}, \quad \hat{R} \rightarrow \lambda \hat{R}, \quad g_{Y M} \rightarrow \frac{g_{Y M}}{\lambda}$,

3. $M \rightarrow \lambda^{2} M, \quad h \rightarrow \frac{h}{\lambda}, \quad \phi \rightarrow \lambda \phi, \quad r \rightarrow \lambda r$,

y permiten escoger $R=r_{h}=1$ y fijar el valor de $h(r)$ en el borde a $h(\infty)=1$. El comportamiento en el IR de estas ecuaciones es el mismo que para un agujero negro cargado

$$
\begin{aligned}
M & =M_{1}\left(r-r_{h}\right)+M_{2}\left(r-r_{h}\right)^{2}+\ldots \\
h & =h_{0}+h_{1}\left(r-r_{h}\right)+h_{2}\left(r-r_{h}\right)^{2}+\ldots \\
\omega & =\omega_{0}+\omega_{1}\left(r-r_{h}\right)+\omega_{2}\left(r-r_{h}\right)^{2}+\ldots \\
\phi & =\phi_{1}\left(r-r_{h}\right)+\phi_{2}\left(r-r_{h}\right)^{2}+\ldots
\end{aligned}
$$

en donde, como antes, se impuso que el potencial de Maxwell $\phi$ se anule en el horizonte con el objeto de obtener un campo de gauge bien definido cuando se realice la continuación Euclídea. En el UV se pedirá

$$
\begin{aligned}
M & =r^{2}+2 h_{1}^{b} r+\left(h_{1}^{b}\right)^{2}+\frac{M_{1}^{b}}{r}+\frac{-8 h_{1}^{b} M_{1}^{b}+\rho^{2}+2\left(\omega_{1}^{b}\right) / 3}{8 r^{2}}+\ldots \\
h & =1+\frac{h_{1}^{b}}{r}-\frac{\left(\omega_{1}^{b}\right)^{2}}{48 r^{4}}+\ldots \\
\omega & =\frac{\omega_{1}^{b}}{r}-\frac{h_{1}^{b} \omega_{1}^{b}}{r^{2}}+\ldots \\
\phi & =\mu+\frac{\rho}{r}-\frac{\rho h_{1}^{b}}{r^{2}}+\ldots
\end{aligned}
$$

Nótese que para que ocurra una ruptura espontánea de simetría no se permite un término no normalizable en $\omega$. Como antes, las simetrías de escala (3) permiten fijar $h_{0}^{b}=1$. En la figura 3.6 se muestra el comportamiento de las solución y en la figura 3.7 se grafica el parámetro de orden $\left\langle J_{x}^{1}\right\rangle>\propto \omega_{1}^{b}$ como una función de la temperatura. Para $T=T_{c}$ ambos condensados se anulan y ocurre una transición de fase de segundo orden. Nótese que los valores del condensado para el caso del $p+i p$ son menores que aquellos para el superconductor tipo $p$. 


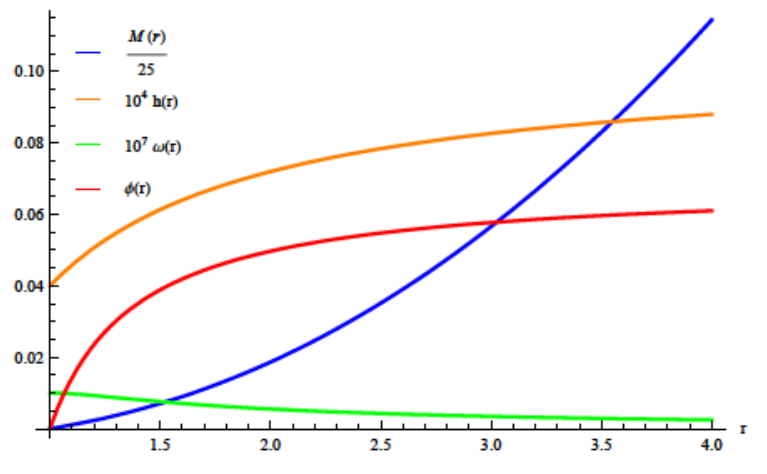

Figura 3.6: Comportamiento de las funciones adimensionales $M(r), h(r), \omega(r)$ y $\phi(r)$, para $g_{Y M}=2, T=0,2312 \mu$.

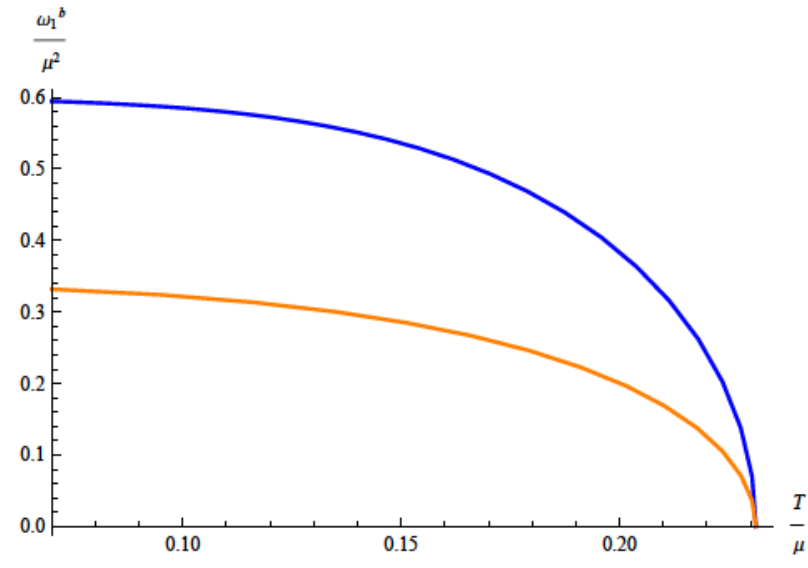

Figura 3.7: El valor de expectación $\left\langle J_{x}^{1}\right\rangle \propto \omega_{1}^{b}$ de la teoría de campos dual como función de la temperatura para el superconductor tipo $p$ (línea azul) y tipo $p+i p$ (línea naranja) para $g_{Y M}=2$. Se anula para $T>T_{c}=0,2312$, lo cual sugiere la existencia de una transición de fase entre el estado superconductor y el normal.

\section{Termodinámica}

La temperatura asociada a la solución de fondo es proporcional a la derivada de la componente $g_{t t}$ de la métrica evaluada en el horizonte. En este caso se tiene

$$
T=\frac{M_{1}}{2 \pi}
$$

La formula de Bekenstein-Hawking, que relaciona la entropía con el area del horizonte del agujero negro, en este caso es

$$
S=\frac{2 \pi}{\kappa_{(4)}^{2}} A_{h}=\frac{2 \pi}{\kappa_{(4)}^{2}} r_{h}^{2} h_{0}^{2} .
$$

En la figura 3.8 se muestra la entropía en el caso del superconductor tipo $p+i p$ (linea naranja), del tipo $p$ (línea azul) y del agujero negro de RN (línea roja).

El grand potencial $\Omega$ está dado por el coeficiente del término sub-leading de la función $g_{t t}$ de la métrica,

$$
\Omega=\frac{V M_{1}^{b}}{\kappa_{(4)}^{2}}
$$

Este potencial se dibuja en la figura 3.9, en donde claramente se muestra que para cualquier valor de la temperatura la solución tipo $p$ (azul) es preferida por sobre la $p+i p$ (naranja) debido a su menor energía libre. Para $T>T_{c}$ el sistema se encuentra en su fase normal (en rojo) y el condensado se anula (ver figura 3.7). 


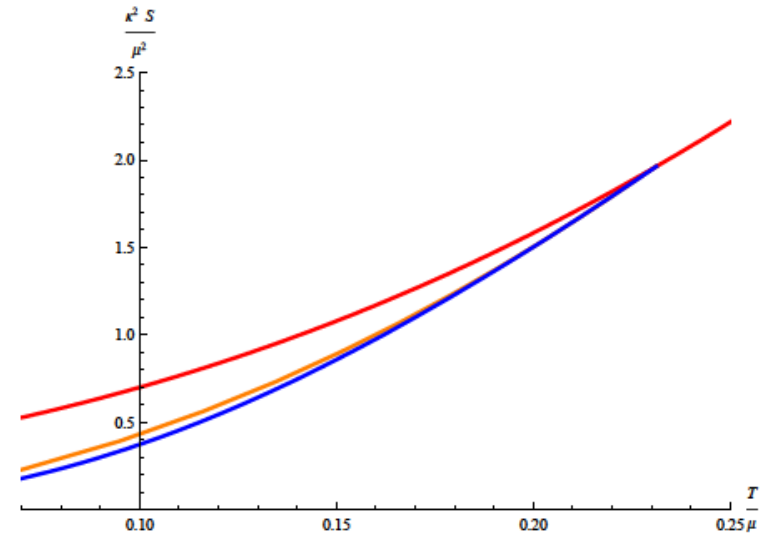

Figura 3.8: Entropía de Bekenstein-Hawking para el agujero negro de RN (rojo) y las soluciones de superconductor tipo $p$ (azul) y $p+i p$ (naranja) con $g_{Y M}=2$ y $T_{c}=0,2312$. Hay una transición de fase de segundo orden para ambos superconductores en $T=T_{c}$.

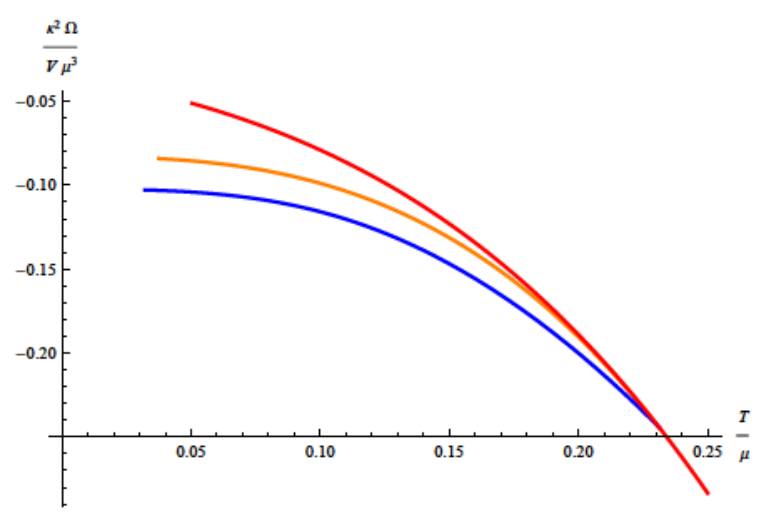

Figura 3.9: Potencial gran canónico como función de la temperatura. Solución de RN (rojo), $p+i p$ (naranja) y $p$ (azul). Para todo rango de temperaturas por debajo de $T_{c}$ la solución de superconductor tipo $p$ es preferida por sobre la $p+i p\left(g_{Y M}=2\right.$ y $\left.T_{c}=0,2312\right)$.

\subsection{Entropía de entrelazamiento}

Una prescripción holográfica para calcular la entropía de entrelazamiento (EE) en el espacio $\mathrm{AdS}_{d+1}$ dual a una $\mathrm{CFT}_{d}$ fue propuesta en [62] en términos de superficies mínimas. La prescripción involucra subdividir el sistema en dos regiones, $\mathcal{A}$ y su complemento $\mathcal{B}$, y encontrar las superficie estática mínima $d-1$ dimensional (a tiempo constante) $\gamma_{\mathcal{A}}$ tal que su borde coincide con el borde del subsistema $\mathcal{A}$ (ver figura 3.10).

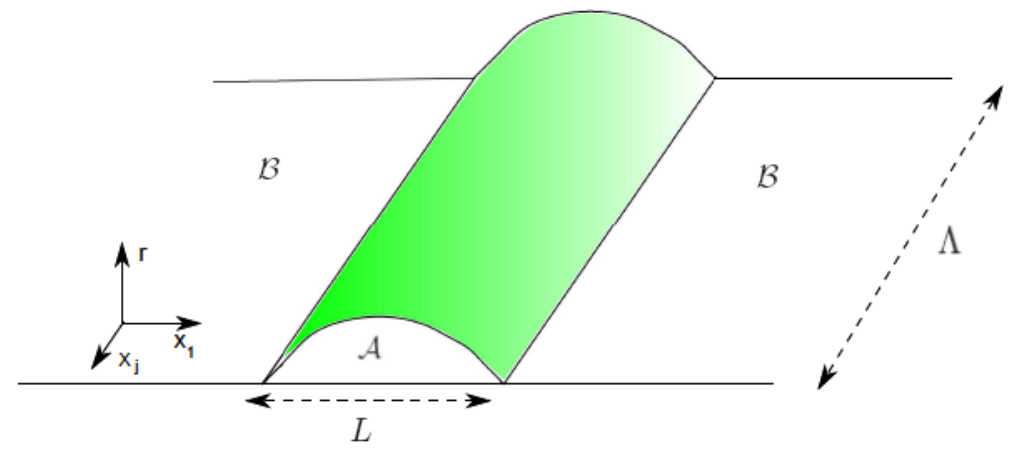

Figura 3.10: Diagrama de la región en forma de banda $\mathcal{A}$ usada para calcular la entropía de entrelazamiento.

La entropía de entrelazamiento entre las dos regiones es el area clásica de $\gamma_{\mathcal{A}}$,

$$
\mathcal{S}_{\mathcal{A}}=\frac{2 \pi}{\kappa_{(d+1)}^{2}} \int_{\gamma_{\mathcal{A}}} d^{(d-1)} \sigma \sqrt{g_{\text {ind }}^{(d-1)}}
$$

en donde $g_{i n d}^{(d-1)}$ es la métrica inducida sobre la superficie y $\kappa_{(d+1)}^{2}$ es la constante gravitatoria en $d+1$ dimensiones. 
En [87] y [88] la EE fue calculada para fondos gravitatorios duales a teorías de gauge confinantes y a geometrías de agujero negro respectivamente. En esta sección se realizará un cálculo similar para un fondo de gravedad general y se aplicará la prescripción a las soluciones de superconductores tipo $p$ y $p+i p$ encontrados en la sección 3.1. Nótese que la prescripción es muy similar a la realizada en $[85,86]$ para calcular valores de expectación de lazos de Wilson.

Escribamos la métrica de fondo $d+1$ dimensional como

$$
d s_{d+1}^{2}=-g_{t t}(r) d t^{2}+g_{x_{i} x_{i}}(r) d x_{i}^{2}+g_{r r}(r) d r^{2}, \quad i=1 \ldots d-1,
$$

en donde $r$ es la coordenada holográfica. La región de interés será una banda recta en la dirección $x_{j}$ con ancho $L$ en la dirección $x_{1}$. La forma de embeber la superficie estáticamente es $x_{1}=x_{1}(\zeta), x_{j}=\zeta_{j}, r=r(\zeta)$, con $j=2, \ldots, d-1$. Aún se tiene invarianza ante difeomorfismos en $\mathcal{S}_{\mathcal{A}}$, dependiendo del contexto esta puede ser fijada como $x_{1}=\zeta$ (embedding global) o $r=\zeta$. La entropía (3.30) es

$$
\mathcal{S}_{\mathcal{A}}=\frac{2 \pi \Lambda}{\kappa_{(d+1)}^{2}} \int d \zeta \sqrt{g_{x_{2} x_{2}}(r) \ldots g_{x_{d-1} x_{d-1}}(r)} \sqrt{g_{r r}(r) r^{\prime 2}+g_{x_{1} x_{1}}(r) x_{1}^{\prime 2}},
$$

en donde $\Lambda=\int d \zeta_{2} \ldots d \zeta_{d-1}$ y el símbolo ' denota derivadas en $\zeta$.

Definiendo $g_{\chi \chi}(r)=g_{x_{2} x_{2}}(r) \ldots g_{x_{d-1} x_{d-1}}(r)$ y las funciones

$$
f^{2}(r)=g_{\chi \chi}(r) g_{x_{1} x_{1}}(r), \quad \eta^{2}(r)=g_{\chi \chi}(r) g_{r r}(r)
$$

la entropía de entrelazamiento se puede escribir

$$
\mathcal{S}_{\mathcal{A}}=\frac{2 \pi \Lambda}{\kappa_{(d+1)}^{2}} \int d \zeta \sqrt{\eta^{2}(r) r^{\prime 2}+f^{2}(r) x_{1}^{\prime 2}}
$$

Minimizando (3.34) se obtiene

$$
x_{1}^{\prime}(\zeta)= \pm \frac{f\left(r_{0}\right) \eta(r)}{f(r)} \frac{r^{\prime}(\zeta)}{\sqrt{f^{2}(r)-f^{2}\left(r_{0}\right)}},
$$

en donde $r=r_{0}$ es el valor mínimo en la coordenada holográfica alcanzada por la superficie. Dependiendo del fondo gravitatorio estudiado este punto podría ser el radio del horizonte o el fin del espacio-tiempo. Invirtiendo esta relación se puede leer la longitud de la banda en la dirección $x_{1}$

$$
L=2 \int_{r_{0}}^{\infty} d r \frac{d x_{1}}{d r}=2 \int_{r_{0}}^{\infty} d r \frac{\eta(r)}{f(r)} \frac{f\left(r_{0}\right)}{\sqrt{f^{2}(r)-f^{2}\left(r_{0}\right)}} .
$$

Ahora se fijará la invarianza ante difeomorfismo como $x_{1}(\zeta)=\zeta$, esta elección tiene la ventaja de proveer una parametrización completa de $r\left(x_{1}\right),\left(x_{1} \in\left[-\frac{L}{2}, \frac{L}{2}\right]\right.$ y las condiciones de borde son $r\left( \pm \frac{L}{2}\right)=\infty$ ). A partir de (3.35) en (3.34) la entropía de entrelazamiento queda

$$
\mathcal{S}_{\mathcal{A}}\left(r_{0}\right)=2 \frac{2 \pi \Lambda}{\kappa_{(d+1)}^{2}} \int_{r_{0}}^{\infty} d r \frac{f(r) \eta(r)}{\sqrt{f(r)^{2}-f\left(r_{0}\right)^{2}}} .
$$

la expresión (3.37) diverge en $r=\infty$ debido a la extensión infinita de la superficie. Esta divergencia se entiende a partir de la existencia de otra solución, con las mismas condiciones 
de borde, consistente de dos superficies desconectadas que se extienden a lo largo de toda la dirección radial. $\mathrm{Su}$ area es

$$
\mathcal{S}_{\mathcal{A}_{\text {disc }}}=2 \frac{2 \pi \Lambda}{\kappa_{(d+1)}^{2}} \int_{r_{\text {min }}}^{\infty} d r \eta(r)
$$

aquí $r_{\text {min }}$ es el valor mínimo de $r$ permitido por la geometría. La EE queda definida con respecto al estado de referencia (3.38)

$$
\Delta \mathcal{S}_{\mathcal{A}}=\frac{4 \pi \Lambda}{\kappa_{(d+1)}^{2}}\left(\int_{r_{0}}^{\infty} d r \frac{f(r) \eta(r)}{\sqrt{f(r)^{2}-f\left(r_{0}\right)^{2}}}-\int_{r_{\text {min }}}^{\infty} d r \eta(r)\right) .
$$

En lo que sigue vamos a estudiar la EE para las soluciones de (3.10) y (3.1.2).

En el caso del superconductor tipo $p$ las funciones relevantes son

$$
f_{p}^{2}(r)=g_{y y} g_{x x}=r^{4}, \quad \eta_{p}^{2}(r)=g_{y y} g_{r r}=\frac{r^{2}}{h^{2} N}
$$

en donde el subíndice $p$ nos recuerda que corresponden al tipo $p$. Con esto, podemos calcular explícitamente la cantidad (3.39)

$$
\Delta \mathcal{S}_{\mathcal{A}}=\frac{4 \pi \Lambda}{\kappa_{(4)}^{2}}\left(\int_{r_{0}}^{\infty} d r \frac{r^{3}}{h \sqrt{N} \sqrt{r^{4}-r_{0}^{4}}}-\int_{r_{\text {min }}}^{\infty} d r \frac{r}{h \sqrt{N}}\right)
$$

En la figura 3.11 se muestra $\Delta \mathcal{S}_{\mathcal{A}}$ como función de la longitud de la banda $L$. Se graficó para diferentes valores del parámetro de deformación y diferentes valores de la temperatura. Como era esperado, la curva inferior es la que tiene menor temperatura debido a que al bajar la temperatura hay más grados de libertad condensados. El comportamiento lineal para valores grandes de $\mu L$ es una manifestación de la ley de area propuesta en (3.30). La figura 3.13 muestra la EE de las fases condensadas (línea azul) y normal (linea roja) como función de la temperatura y para un valor constante de $L$.

Con el objetivo de obtener una entropía finita sin sustraer la solución desconectada, podemos escribir la EE de la siguiente manera

$$
\mathcal{S}_{\mathcal{A}}\left(r_{0}\right)=\frac{4 \pi \Lambda}{\kappa_{(4)}^{2}} \int_{r_{0}}^{\mathcal{R}} d r \frac{r^{3}}{h \sqrt{N} \sqrt{r^{4}-r_{0}^{4}}}=S_{\mathcal{A}}+\frac{4 \pi \Lambda}{\kappa_{(d+1)}^{2}} \mathcal{R}
$$

en donde $S_{\mathcal{A}}$ tiene dimensiones de longitud y no presenta divergencias. La figura 3.13 muestra que la EE para el superconductor (linea azul) es menor que para la solución de RN (linea roja). Como se mencionó anteriormente esto es esperable debido que la solución superconductora tiene grados de libertad condensados.

Realizando el mismo análisis para la solución de (3.1.2) se obtiene

$$
f_{p+i p}^{2}(r)=g_{y y} g_{x x}=r^{4} h^{4}, \quad \eta_{p+i p}^{2}(r)=g_{y y} g_{r r}=\frac{r^{2} h^{2}}{M}
$$

y la siguiente EE

$$
\Delta \mathcal{S}_{\mathcal{A}}=\frac{4 \pi \Lambda}{\kappa_{(4)}^{2}}\left(\int_{r_{0}}^{\infty} d r \frac{r^{3} h^{3}}{\sqrt{M} \sqrt{r^{4} h^{4}-r_{0}^{4} h\left(r_{0}\right)^{4}}}-\int_{r_{m i n}}^{\infty} d r \frac{r h}{\sqrt{M}}\right)
$$




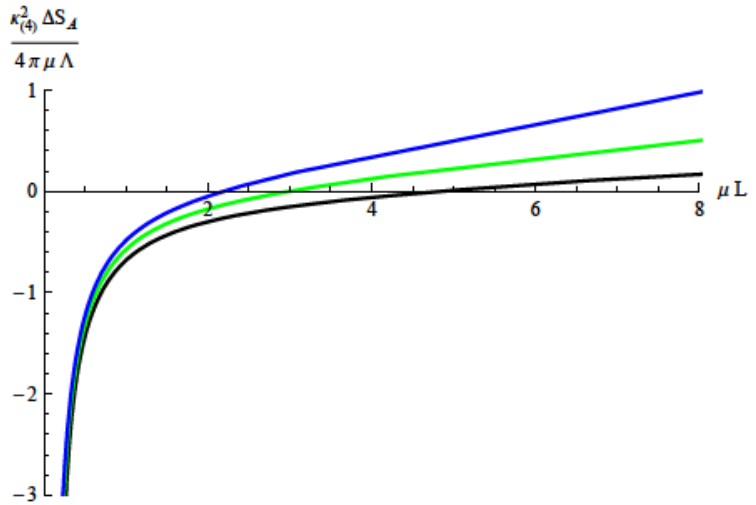

Figura 3.11: Entropía de entrelazamiento como función del tamaño de la banda para la solución tipo $p$. Las curvas negra, verde y azul corresponden a $g_{Y M}=1, T=0,0749 \mu, g_{Y M}=1,5, T=$ $0,1565 \mu$ у $g_{Y M}=2, T=0,2312 \mu$ respectivamente.

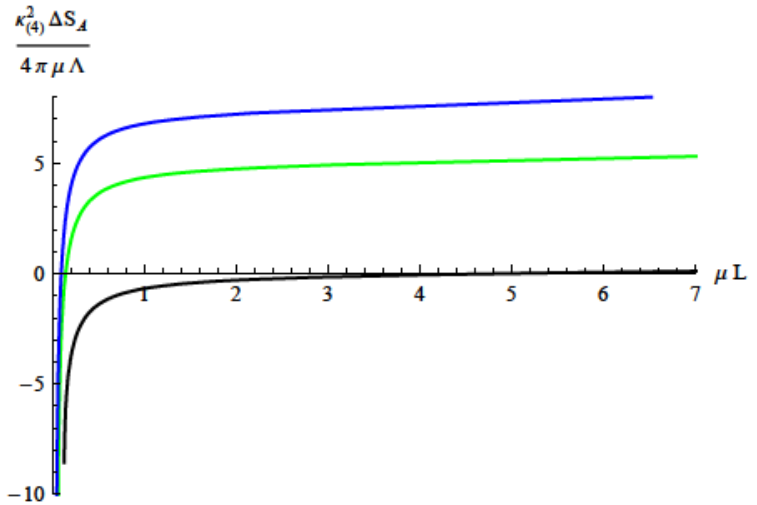

Figura 3.12: Entropía de entrelazamiento como función del tamaño de la banda para la solución tipo $p+i p$. Las curvas negra, verde y azul corresponden a $g_{Y M}=1, T=0,0749 \mu, g_{Y M}=$ $1,5, T=0,1565 \mu$ y $g_{Y M}=2, T=$ $0,2312 \mu$ respectivamente.

La figura 3.12 muestra el comportamiento de $\Delta \mathcal{S}_{\mathcal{A}}$ para este caso, y podemos realizar el mismo análisis que para el caso del superconductor $p$. De nuevo, podemos utilizar un enfoque diferente para obtener la entropía no divergente sin realizar la sustracción de las soluciones desconectadas. Esto consiste en separar la parte divergente de la integral (3.37) y tener en cuenta su parte finita, $S_{\mathcal{A}}$. En este caso

$$
\mathcal{S}_{\mathcal{A}}\left(r_{0}\right)=\frac{4 \pi \Lambda}{\kappa_{(4)}^{2}} \int_{r_{0}}^{\mathcal{R}} d r \frac{r^{3} h^{3}}{\sqrt{M} \sqrt{r^{4} h^{4}-r_{0}^{4} h\left(r_{0}\right)^{4}}}=S_{\mathcal{A}}+\frac{4 \pi \Lambda}{\kappa_{(4)}^{2}} \mathcal{R} .
$$

La figura 3.13 muestra esta parte finita (linea naranja) para $g_{Y M}=2$ y $T_{c}=0,2312$, de donde se ve que, como se esperaba, es menor que la EE de la solución de RN. Además la figura muestra que la EE en el caso del superconductor tipo $p$ es menor que para el $p+i p$. Esto sugiere que a una dada temperatura hay más grados de libertad condensados en el superconductor $p$.

\subsubsection{Conclusiones}

En este capítulo se estudiaron los duales holográficos a superconductores tipo $p$ y $p+i p$ en $3+1$ dimensiones. Se calculó la deformación de la geometría dual al tipo $p$ y sus propiedades termodinámicas. Como era esperable, y en contraste a la solución en $4+1$ dimensiones de [66], se encontró una transición de fase de segundo orden entre las fases normal y superconductora. Luego, se resumió la deformación producida por el campo de gauge sobre la geometría del $p+$ ip y se comparó con nuestra solución. A partir del estudio de las cantidades termodinámicas y en particular de sus potencial gran canónico, se notó que para un valor fijo de la temperatura la solución tipo $p$ tiene menor potencial y por lo tanto es la preferida por el sistema. Esto se relaciona con el echo de que la solución tipo $p+i p$ es inestable frente a pequeñas fluctuaciones y decae a la solución tipo $p$. 


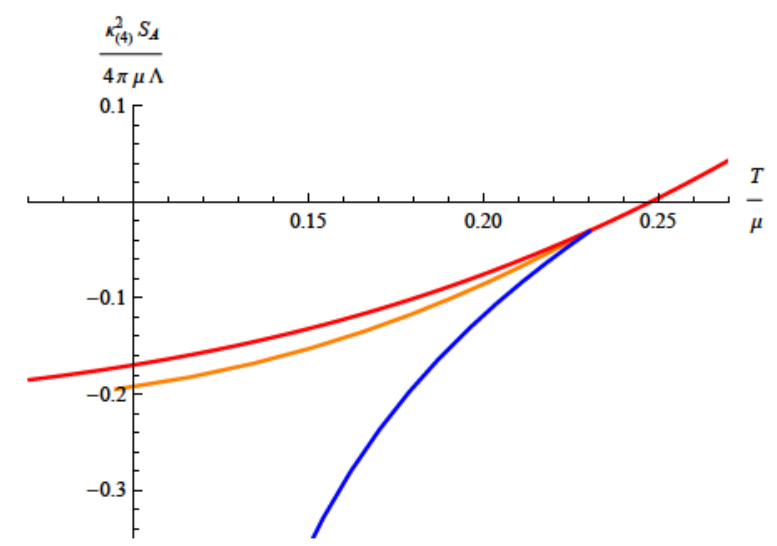

Figura 3.13: Entropía de entrelazamiento como función de la temperatura y a $\mu L=3$ fijo. La curva azul es para la solución tipo $p$, la naranja para la tipo $p+i p$ y la roja para $\mathrm{RN}$. Se utilizó $g_{Y M}=2$ y $T_{c}=0,2312$.

Finalmente, usando la propuesta holográfica estudiada en [62], se calculó la entropía de entrelazamiento de la teoría cuántica de campos usando su dual gravitatorio, para una geometría de banda y como función de la temperatura y el tamaño de la banda. Se observó que en ambos casos la EE se comporta lineal para valores grandes de $L$, lo cual confirma la ley de area propuesta. La EE vs L tiende a valores mas grandes de $\Delta \mathcal{S}_{\mathcal{A}}$ a medida que se incrementa su temperatura. Como función de la temperatura se observa que la mayor EE es para la solución de RN. Esto era esperable debido a que el superconductor tiene grados de libertad condensados. Además la solución tipo $p$ presenta más grados de libertad condensados que el $p+i p$. Esto podría explicar el hecho de que el valor del condensado para el tipo $p$ es más grande que para el $p+i p$ a una dada temperatura. 


\title{
Capítulo 4
}

\section{Bosones de Goldstone tipo II holográficos}

\author{
"Why not indeed? Here is one possible answer to that very reasonable question. \\ But please remember: this is only a work of fiction. \\ The truth, as always, will be far stranger."
}

Arthur C. Clarke

El teorema de Goldstone implica la aparición de un modo sin gap cada vez que una simetría continua sea rota. En general no dice nada de la forma precisa de la relación de dispersión ni que implique un modo no masivo por cada generador roto. Es un hecho bien establecido que aun para teorías de campos relativistas en presencia de un potencial químico, modos de Goldstone con relación de dispersión cuadrática, Los modos de Goldstone tipo II, aparecen en el espectro. Realizamos dos modelos holográficos que realizan modos de Goldstone tipo II en su espectro de modos quasinormales. Los modos están basados en generalizaciones con simetría $U(2)$ del bien estudiado superfluido s-wave. Nuestros resultados incluyen modos de Godstone sin generadores rotos pero con una realización de la simetría inusual y conductividades con una dependencia en frecuencia con una notable similitud con la del grafeno. Los resultados de esta sección fueron presentados en [89].

\subsection{Introducción}

La fortaleza de la correspondencia gauge/gravedad es que permite estudiar la dinámica de tiempo real de teorías de campos fuertemente acopladas de manera bastante sencilla. La teoría de respuesta lineal permite calcular el comportamiento del sistema luego de una pequeña perturbación. También es aplicable al régimen de tiempos largos de una perturbación grande cuando se amortiguó lo suficiente y entra en el régimen lineal. El ingrediente básico de la teoría de respuesta linal en la función de Green retardada. En el contexto de la correspondencia AdS/CFT, en [94, 95] se mostró cómo calcular funciones de Green retardadas imponiendo condiciones de contorno entrantes en los horizontes de agujeros negros. Para agujeros negros con horizontes no degenerados las funciones de Green retardadas son analíticas en el semiplano superior de la frecuencia compleja y tienen una serie infinita de polos aislados en el semiplano inferior. Estos polos son los modos quasinormales (QNM) holográficos del agujero negro [96, 97, 98, 99]. Dentro del espectro de QNM, los modos no 
masivos tienen un rol especial, ya que dan la contribución dominante a la función de Green a pequeños momento y frecuencia. Siendo así, determinan la descripción hidrodinámica del sistema. En este capítulo estudiaremos el espectro de QNM de una generalización multicomponente del superfluido s-wave holográfico non-backreacted con particular atención en los modos no masivos.

El estudio de QNM para el superfluido s-wave $U(1)$ fue llevado a cabo en [100]. Como la física básica de la superfluidés es la de la roptura espontánea de simetría es de esperarse que resultados básicos, como el de la existencia de un bosón de Goldstone ${ }^{1}$ continua en superfluidos holográficos. De hecho, uno de los principales resultados de [100] fue que el espectro de QNM en la fase superfluida contiene dicho modo de Goldstone no masivo con relación de dispersión $\omega= \pm v_{s} k+O\left(k^{2}\right)$. Este modo puede ser entendido como el modo de sonido del superfluido y $v_{s}$ como la velocidad del sonido ${ }^{2}$. En el modelo no backreactado, estos son los únicos modos de sonido presentes en la fase rota. En la fase no rota en cambio, existe un único modo hidrodinámico señalando el usual ocmportamiento difusivo de un fluido normal. Su relación de dispersión es $\omega=-i D k^{2}$, donde $D$ es la constante de difusión. Qué sucede con este modo difusivo en la fase rota fue investigado en [100]: Desarrolla un gap imaginario puro $\omega=-i \gamma-i \tilde{D} k^{2}$. Esto es bastante natural, porque un modo solo no se puede mover del eje imaginario ${ }^{3}$. La hidrodinámica de la fase rota está completamente capturada por los modos de Goldstone y los modos de difusión hacen lo más simple que se les puede ocurrir abandonar le régimen hidrodinámico aumentando su gap $\gamma$. Como este modo imaginario puro tiene su origen en el modo univeral difusivo de la fase normal esperamos que sea una característica universal de una gran clase de superfluidos, no solamente holográficos. Este modo necesariamente dominará la respuesta a tiempos grandes del parámetro de orden para perturbaciones homogéneas y en regimenes cerca pero por debajo de la temperatura crítica donde el gap $\gamma$ es bastante pequeño. Entonces el parámetro de orden está condicionado a mostrar un comportamiento puramente exponencial hacia su valor de equilibrio sin ninguna oscilación. En contraste, para temperaturas más bajas donde $\gamma$ se hace más grande hay otros QNMs pequeños con parte real e imaginaria en su frecuencia. En este régimen de bajas temperaturas la respuesta del parámetro de orden será una oscilación amortiguada. Este aspecto universal de la respuesta a tiempos grandes de superfluidos fuen también enfatizada en estudios numéricos recientes de quenches en superfluidos holográficos [102].

En este capítulo generalizamos los resutados de QNM para superfluidos con simetría $U(2)$. En el primer modelo simplemente agregamos un segundo campo escalar con la misma masa, lo llamaremos el modelo ungauged. El segundo modelo también incluye los campos de gauge para la simetría $U(2)$. La diferencia entre estos modelos es la siguiente: En el modelo ungauged sólo la siemtría $U(1)$ es local en el bulk. Tiene sin embargo una simetría $S U(2)$

\footnotetext{
${ }^{1}$ También son llamados frecuentemente bosones de Nambu-Goldstone. Por simplicidad nos referiremos a ellos como bosones de Goldstone o modos de Goldstone a lo largo del texto.

${ }^{2}$ En [101] fue señalado que corresponde al cuarto sonido. Mientras que el segundo sonido a travez de oscilaciones en la temperatura el cuarto sonido es el fenómeno de porpagación del sonido en la fase superfluida solamente [124]. Esto es que el modo sobrevive al límite probe en el cual la propagación en la compoente normal del fluido dual está prohibida. En este modelo no hay otro modo de sonido, así que hablaremos del Goldstone sencillamente como modo de sonido (sound mode). Debido a su naturaleza de cuarto sonido, interpola entre el segundo sonido a $T=T_{c}$ y el sonido normal a $T=0$.

${ }^{3}$ Los QNM están restringidos a venir en pares $\omega_{n}$ y $\tilde{\omega}_{n}=-\omega_{n}^{*}$ o a estar fijos en el eje imaginario. Esto viene de consideraciones bastante generales asociadaas a funciones de Green retardadas, ver apéndice A.1.
} 
global ${ }^{4}$ bajo la cual los campos escalares transforman como doblete. De acuerdo con el diccionario holográfico, este modelo contiene tan solo una corriente conservada, correspondiente al único campo de gauge en el bulk. La teoría de campos dual hereda por supuesto la simetría $S U(2)$ global del bulk, pero esta simetría no es generada por operadores en la teoría de campos conforme dual. Esto es parecido al límite de desacoplamiento en el que estamos trabajando y en el cual las fluctuaciones de la métrica están suprimidas. La teoría de campos dual en este caso, no tiene tensor de energía momento, estrictamente hablando. En teorías usuales lagrangianas en 4 dimensiones, el teorema de Noether garantiza que siempre podemos construir una carga conservada generando una dada simetría del lagrangiano. En teorías definidas holográficamente la existencia de un lagrangiano tetradimensional no está garantizada a priori y entonces el teorema de Noether no se aplica inmediatamente. Este es el caso acá. Aunque la teoría dual tiene la simetría $S U(2)$ (y covariancia de Poincaré) no tiene operadores generando estas simetrías. Podemos hablar de dichas simetrías como un automorfismo externo del álgebra de operadores de la teoría dual de campos ${ }^{5}$. Físicamente la diferencia entre ambos modelos es que el ungauged es un fluido de una componente (sólo hay una noción de carga) mientras que el gauged es un fluido de dos componentes. En el último, las cargas son los valores de espectación de la componente cero de las corrientes en el subálgebra de Cartan de la simetría $U(2)$.

Aunque este modelo ungauged no contiene corrientes conservadas para la simetría $S U(2)$ y entonces muchas de las pruebas estándar sobre la existencia de bosones de Goldstone no aplicarían estrictamente, encontramos un nuevo modo no masivo en el espectro de QNMs. Este modo sin embargo no es un bosón de Goldstone estándar con relación de dispersión lineal sino un modo de Goldstone tipo II cuya energía depende de manera cuadrática con el momento.

El segundo modelo que consideramos tiene un doblete escalar acoplado al conjunto completo de campos de gauge de $U(2)$. Prendemos un campos de gauge solamente para la simetría $U(1)$ overall. Entonces a altas temperaturas el sistema goza de la simetría $U(2)$ completa. A bajas temperaturas esta simetría es rota a $U(1)$. En este modelo la teoría dual tiene operadores generando toda la simetría $U(2)$. Podemos por lo tanto también estudiar las conductividades.

En el contexto de materia condensada fue argumentado hace tiempo en [103] superfluidos multicomponentes tienen modos de Goldstone inusuales con relaciones de dispersión cuadráticas. En el contexto de altas energías este tipo de sistemas fueron considerados para modelar condensación de Kaones en la color-flavor locked phase de QCD en [104, 105] una vez más enfatizando en la existencia de modos de Goldstone con relaciones de dispersión cuadráticas. Nuestro modelo holográfico gaugeado es un análogo inmediato holográfico del modelo [104, 105] y de hecho nosotros también encontramos modos de Goldstone con relaciones de dispersión cuadráticas. Vale decir que en el contecto de holografía un modo de Goldstone del tipo II fue encontrado en el contexto de teoría de defectos usando D3/D5 magnetizadas

\footnotetext{
${ }^{4}$ Aunque no es de esperar que simetrías globales sean consistentes con una truncación de una teoría de gravedad cuántica no pueden ser obtenidas en ciertos límites de decompactificación en teoría de cuerdas: e.g. enrollando branas en ciclos y luego tomando el volumen del ciclo a infinito de manera tal que el acoplamiento de gauge en las branas va a cero dejando solo una simetría global en las mismas.

${ }^{5}$ Un ejemplo de teoría de cuerdas de una situación semejante es la teoría basada en el pequeño álgebra superconforme $\mathcal{N}=4$ en la hoja de mundo. Este álgebra posee una simetría $S O(4)=S U(2) \times S U(2)$ grande actuando en cuatro supercargas de las cuales sólo un $S U(2)$ está representado mediante corrientes quirales en la hoja de mundo.
} 
[106].

Recolectemos ahora algunos de los resultados importantes sobre bosones de Goldstone (Un review archiútil acerca de rotura de simetría y bosones de Goldstone es [107]). Primero tenemos por supuesto el teorema de Goldstone propiamente dicho. Su prueba asume la existencia de una corriente conservada $j^{\mu}$ tal que al ccarga conservada sea $Q=\int d^{d} x j^{0}$ (con $d$ dimensiones espaciales). El teorema entonces nos dice que la rotura espontánea de una simetría global implica la existencia de un modo cuya energía satisface

$$
\lim _{k \rightarrow 0} \omega(k)=0 .
$$

El teorema por sí mismo no dice nada sobre el número de modos, ni fija la dependencia en $k$ de la frecuencia. En presencia de simpetría de Poincaré uno puede hacer sin embargo una afirmación más fuerte, que el número de modos no masivos tiene que igualar al número de generadores rotos y que su energía debe ir lineal con el momento.

La simetría de Lorentz puede estar ausente sin embargo, ya sea en principio como es el caso de teorías de campos no relativistas o el sistema bajo consideración puede estar en un estado que rompa simetría de Lorentz de manera explícita, tal como estar a densidad de carga finita. En estos casos otro teorema clasifica a los bosones de Goldstone como tipo I si su enegía se anula como una potencia impar del momento o como tipo II si su energía se anula como una potencia par del momento en el límite de momento cero. El número de modos de Goldstone de tipo I y II debe cumplir

$$
n_{I}+2 n_{I I} \geq N_{B G}
$$

donde $N_{B G}$ es el número de generadores rotos [108]. El número de bosones de tipo I y II puede ser restringido aun más. Asumiendo que los generadores rotos satisfacen $\left\langle\left[Q_{a}, Q_{b}\right]\right\rangle=B_{a b}$ el número de bosones de Goldston cumple que [109, 110, 112] (ver también [107, 113, 111] para más información sobre le conteo de Goldstones).

$$
n_{I}+n_{I I}=N_{B G}-\frac{1}{2} \operatorname{rank}(B) .
$$

Debemos considerar ahora el patrón de rotura de simetría de la teoría del borde dual al modelo holográfico gaugeado. Veremos que a travez de los teoremas enunciados arriba la existencia de un bosón de Goldstone de tipo dos está garantizada. Tenemos en total cuatro generadores de simetría. La simetría está rota de $U(2)$ a $U(1)$ lo que implica tener tres generadores rotos. En la fase rota las cargas correspondientes al $U(1)$ overall y al generador de Cartan $U(1)$ dentro de $S U(2)$ reciben valores de espectación de vacío. Entonces el rango de la matriz $B$ es dos y así también el número de bosones de Goldstone de tipo I y de tipo II deberían sumar a dos. Esto es precisamente lo que encontramos en el espectro de QNM, un modo no masivo con relación de dispersión lineal y un modo masivo con relación de dispersión cuadrática.

Notamos también que el modelo ungauged satisface también el teorema de Goldstone y la regla de conteo de Chadha y Nielsen (4.2). Viola sin embargo la regla de conteo refinada de (4.3). En un sentido estricto este modelo teine un sólo generador ya que tiene tan sólo un $U(1)$ campo de gauge en el bulk. entonces la regla de conteo (4.3) sugeriría la existencia de tan sólo un bosón de Goldstone, el número de generadores rotos es uno y la matriz $B$ se anula trivialmente. 
Este capítulo se organiza de la siguiente manera. En la sección 4.2 repasamos brevemente un modelo de teoría de campos realizando modos de Goldstone del tipo II. Este modelo fue introducido en el contexto de condensación de Kaones en color-flavor locked QCD. Nos sirve de inspiración para contruir los modelos holográficos.

La sección 4.3 trata el análisis del modelo ungauged. Como el modelo de superconductor s-wave es un sector tanto del modelo gauged como ungauged vamos a repasar brevemente los resultados de [100]. Entonces mostramos que pese a la drástica simplificación, i.e. no gaugear la simetría global $S U(2)$ en el bulk, el modelo presenta bosones de Goldstone del tipo II. Entonces, en este modelo un bosón de Goldstone del tipo II es encontrado como consecuencia de haber roto solo un generador de carga (el asociado a la simetría $U(1)$ ).

En la sección 4.4 el modelo $U(2)$ gaugeado. Entonces analizamos las fluctuaciones linealizadas. Las mismas se descomponen en tres sectores desacoplados. Uno es el ya estudiado superfluido s-wave $U(1)$, otro describe el sector no abeliano en el cual viven los bosones de Goldstone tipo II y el tercero tiene la simetría $U(1)$ ue no fue rota. Procedemos a estudiar las conductividades que ahora se acomodan naturalmente en matrices de dos por dos. Mostramos que las conductividades diagonales presentan funciones delta en el límite de bajas frecuencias y son superconductoras en ese sentido. Más aun, a través de un cambio de base encontramos conductividades con una interesante semejanza con las del grafeno [114]. Más aun, encontramos evidencia de que a más bajas temperaturas $T=0,4 T_{c}$ otra inestabilidad ocurre en llevando a un condensado del tipo p. Entonces estudiamos los modos QNM más bajos. Encontramos modos de Goldstone de tipo II y también estudiamos el sector difusivo. Como hay dos simetrías participando hay dos modos difusivos que se aparean en la fase rota, para alejarse así del eje imaginario. Entonces la respuesta en este sector no presenta un decaimiento puramente exponencial inducido por el modo de pseudodifusión del sector $U(1)$.

Concluimos en la sección 4.5 con una discusión de nuestros resultados y un repaso sobre posibles extensiones de nuestra investigación sobre modos Goldstone del tipo II.

Finalmente en el apéndice A.1 presentamos algunas propiedades generales de funciones de Green matriciales que limitan las propiedades de QNMs. En el apéndice A.2 contamos detalles técnicos sobre como calcular QNMs en sistemas acoplados.

\subsection{Un modelo de teoría de campos con bosones de Golsd- tone de tipo II}

Motivados por la condensación de Kaones en la color-flavor locked phase de QCD los autores de [104, 105] estudiaron QCD a potencial químico para la extrañeza no nulo. Se mostró que a un potencial químico crítico igual a la masa de los Kaones, la condensación de Kaones ocurre mediante una transición de fase continua. Más aun, un bosón de Goldstone con una relación de dispersión no relativista $\omega \sim p^{2}$ aparece en la fase condensada. Para ilustrar este hecho, ellos consideraron el siguiente toy model (euclídeo):

$$
\mathcal{L}=\left(\partial_{0}+\mu\right) \phi^{\dagger}\left(\partial_{0}-\mu\right) \phi+\partial_{i} \phi^{\dagger} \partial_{i} \phi+M^{2} \phi^{\dagger} \phi+\lambda\left(\phi^{\dagger} \phi\right)^{2},
$$

donde $\phi$ es un doblete escalar complejo,

$$
\phi=\left(\begin{array}{c}
\phi_{1} \\
\phi_{2}
\end{array}\right)
$$


Mientras $\mu<M$ las masas de las cuatro exitaciones de la teoría son las raíces $\omega$ de

$$
(\omega \pm \mu)^{2}=M^{2}
$$

Todas ellas están doblemente degeneradas. Es inmediato verificar que cuando $\mu=M$ la simetría $U(2)$ se rompe y un nuevo vacío debe ser elegido:

$$
\phi=\frac{1}{\sqrt{2}}\left(\begin{array}{l}
0 \\
v
\end{array}\right), \quad \text { with } \quad v^{2}=\frac{\mu^{2}-M^{2}}{\lambda} .
$$

Estudiando las fluctuaciones del doblete $\phi$ alrededor de este background uno encuentra dos modos masivos y dos modos no masivos con relaciones de dipersión:

$$
\begin{aligned}
\omega_{1}^{2} & =\frac{\mu^{2}-M^{2}}{3 \mu^{2}-M^{2}} p^{2}+O\left(p^{4}\right), \\
\omega_{2}^{2} & =6 \mu^{2}-2 M^{2}+O\left(p^{2}\right), \\
\omega_{3}^{2} & =p^{2}-2 \mu \omega_{3}, \\
\omega_{4}^{2} & =p^{2}+2 \mu \omega_{4} .
\end{aligned}
$$

Si nos concentramos en las raíces positivas vemos que $\omega_{1}$ es un modo de Goldstone normal con relación de dispersión lineal. La raíz positiva de (4.10) es

$$
\omega_{3}=\frac{p^{2}}{2 \mu}+O\left(p^{4}\right)
$$

Este es el modo de Goldstone de tipo II. Tiene formalmente una relación de dispersión no lineal. Como la teoría subyacente tiene sin embargo invarianza de Lorentz hay un modo negativo con dispersión cuadrática. Esto viene de la raíz negativa de $\omega_{4}$. Finalmente $\omega_{2}$ y $\omega_{4}$ son modos masivos con

$$
\omega_{4}=2 \mu+O\left(p^{2}\right) .
$$

Como el patrón de rotura de simetría es $U(2) \rightarrow U(1)$ hay tres generadores espontáneamente rotos pero sólo dos modos no masivos en el espectro. Este modelo satisface todas las reglas de conteo presentadas en la introducción. En particular la regla de Chadha-Nielsen (4.2) es exactamente saturada. El rol de $\omega_{4}$ es especial. Es el modo que se apare con el Goldetone de tipo II Goldstone en las relaciones de dispersión (4.10) y (4.11). Se ha arguemntado que este modo es universal y que su energía a momento cero está protegida frente a correcciones cuánticas $[113,115,116]$. El espectro obtenido de este modelo está resumido en la figura 4.1. En nuestros modelos holográficos buscaremos a este modo masivo especial, compañero del Goldstone tipo II. Resultará que nuestros modelos se diferenciarán en este aspecto: solo el modo en el modelo gaugeado presentará esta característica relación lineal con el potencial químico.

Este simple modelo Lagrangiano nos sirve como ejemplo y guía a la hora de construir modelos holográficos con modos de Goldstone tipo II. De hecho podemos usar el mismo tipo de Lagrangiano de materia en el setup holográfico. De acuerdo con el diccionario holográfico usual una simetría local en el bulk se corresponde con una simetría global en el borde. Entonces seremos conducidos de manera natural al un modelo en el cual gaugeamos la simetría $U(2)$ de(4.4) y lo ponemos en un background de AdS Schwarzschild. En orden de 


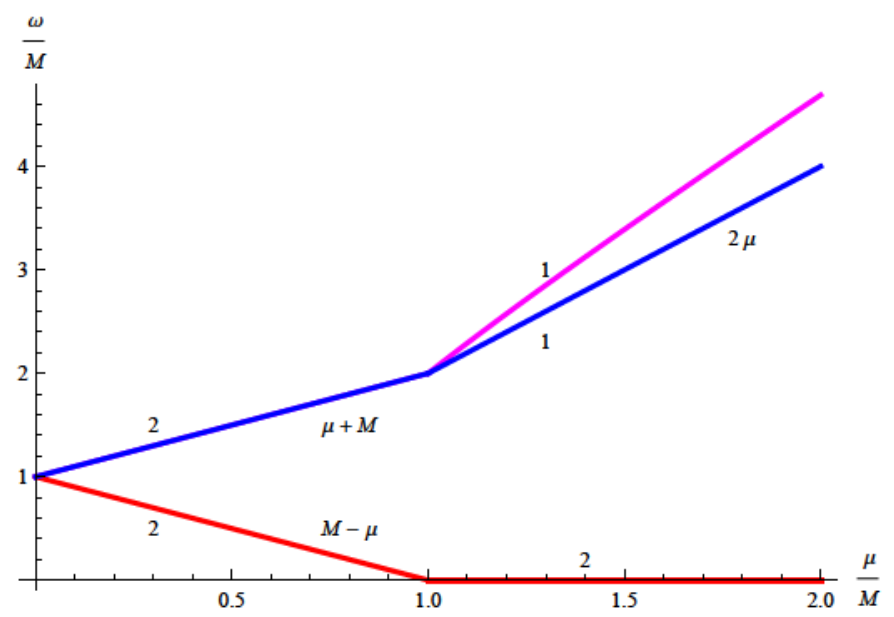

Figura 4.1: El espectro del modelo de teoría de campos. Por debajo del potencial químico crítico $\mu=M$ hay cuatro modos masivos. Las masas son $M-\mu$ y $M+\mu$, los número indican que son doblemente degeneradas. En la fase rota $\mu>M$ hay dos modos de Goldstone con masa cero y dos modos masivos. El modo especial masivo tiene una masa $2 \mu$.

disparar la rotura espontánea de simetría introduciremos un potencial químico en le borde mediante un valor de borde para la componente temporar del overall, campo de gauge $U(1)$ abeliano. Este es entonces nuestro modelo gauged.

De manera alternativa nos podemos preguntar cuáles son los ingredientes mínimos para disparar la rotura espontánea de simetría. El potencial químico recide en el factor $U(1)$ overall. los otros tres campos de gauge de $S U(2)$ no son necesarios para lograr la rotura de simetría. Entonces podemos elegir un modelo minimalista en el cual la simetría $S U(2)$ permanece global en el bulk de AdS. Como mencionamos ya en la introducción esto es altamente controvercial. No hay cargas conservadas asociadas a esta simetría $S U(2)$ sin embargo todos los operadores caen naturalmente en representaciones del grupo ya que no hay nada en el borde que rompa esta simetría global del bulk. Este setup constituye nuestro modelo ungauged y vamos a estudiarlo en más detalle en la próxima sección.

Nótese el siguiente detalle técnico: El modelo de teoría de campos de esta sección se entiende de manera más natural en cuatro dimensiones. En lo que sigue nosotros vamos a estudiar modelos duales a tres dimensiones espaciotemporales para permanecer tan cerca como posible al bien conocido supeorfluido $U(1)$ s-wave de $[27,100]$ explicado en la sección 2.1. Esto sin embargo no es esencial para las caracteríticas relevantes del modelo, i.e. la existencia y naturaleza de los modos hidrodinámicos de Goldstone.

\subsection{El modelo no gaugeado}

Estudiemos ahora el modelo holográfico en el que la condensación de un escalar cargado rompe la simetría $S U(2)$ global en el $b u l k^{6}$. Vamos a mirar el espectro de QNMs a ambos lados de la transición de fase y estudiar sus relaciones de dispersión. Como el simple superfluido

\footnotetext{
${ }^{6}$ Este modelo lleva el nombre de modelo no gaugeado o modelo Bivio. Una de las características sobresalientes del modelo Bivio es que es un paraíso para calcular en comparación al modelo no Bivio, porque como es bien sabido la palabra Bivio añade cualidades paradisíacas a cualquier cosa que describa.
} 
s-wave holográfico $U(1)$ constituye un subsector de este modelo, asì como del ungauged también, aprovecharemos para repasar brevemente las características más salientes de su espectro de QNM.

El modelo mínimo holográfico que contiene bosones de Goldstone tipo II consiste de un doblete escalar de $S U(2)$ cargado bajo un campo de gauge $U(1)$. El Lagrangiano viene dado por

$$
\mathcal{L}=\left(-\frac{1}{4} F^{\mu \nu} F_{\mu \nu}-m^{2} \Psi^{\dagger} \Psi-\left(D^{\mu} \Psi\right)^{\dagger} D_{\mu} \Psi\right)
$$

donde

$$
\Psi=\left(\begin{array}{c}
\lambda \\
\psi
\end{array}\right), \quad D_{\mu}=\partial_{\mu}-i A_{\mu}
$$

y $A_{\mu}$ es el campo de gauge abeliano. Elegimos la masa del escalar $m^{2}=-2 / L^{2}$. Este es básicamente el mismo modelo de [27] con la excepción de que agregamos un segundo campo escalar complejo $\lambda$ con la misma masa. Debido a la degeneración en la masa el modelo posee además de la simetría local $U(1)$ en el bulk una simetría global $S U(2)$. Nótese que la simetría global $S U(2)$ es en principio insuficiente para setear al campo $\lambda(r)=0$. Pero estamo interesados en soluciones estáticas sin fuentes para los campos escalares en el bulk, i.e. asumimos que el modo dominante no normalizable no se enciende. El espacio de soluciones es entonces un espacio vectorial bidimensional complejo caracterizado por los VEVs de los campos escalares de la teoría de c ampos dual. En este espacio de parámetros podemos actuar con la simetría $S U(2)$ global para setear al operador correspodiente al campo $\lambda$ igual a cero. Desde ahora, tanto el modo normalizable como el no normalizable de $\lambda$ está seteados a cero, de manera tal que $\lambda(r)=0$.

Vamos a trabajar en el límite prbe, en el cual el coupling del campo de gauge es muy grande y la backreaction de los campos de materia en la métrica puede ser despreciada. La métrica de background es entonces elegida como la Schwarzschild-AdS black brane

$$
\begin{array}{r}
d s^{2}=-f(r) d t^{2}+\frac{d r^{2}}{f(r)}+\frac{r^{2}}{L^{2}}\left(d x^{2}+d y^{2}\right), \\
f(r)=\frac{r^{2}}{L^{2}}-\frac{M}{r} .
\end{array}
$$

El horizonte del agujero negro está ubicado en $r_{H}=M^{1 / 3} L^{2 / 3}$ y su temperatura de Hawking es $T=\frac{3 r_{H}}{4 \pi L^{2}}$. En lo que sigue, usaremso coordenadas adimensionalizadas, lo que implica

$$
(r, t, x, y) \rightarrow\left(r_{H} \rho, \frac{L^{2}}{r_{H}} \bar{t}, \frac{L^{2}}{r_{H}} \bar{x}, \frac{L^{2}}{r_{H}} \bar{y}\right) .
$$

Estos reescaleos nos permiten setear $M=r_{H}=1$ en el sistema adimensional. Con la intencion de prender un potencial químico finito en la teoría del borde, el campo de Maxwell del bulk

$$
A=\chi(\rho) d \bar{t},
$$

debe tomar un valor no nulo en el borde.

Las ecuaciones de movimiento para los campos de background son

$$
\begin{aligned}
& \chi^{\prime \prime}+\frac{2}{\rho} \chi^{\prime}-\frac{2 \psi^{2}}{f} \chi=0, \\
& \psi^{\prime \prime}+\left(\frac{f^{\prime}}{f}+\frac{2}{\rho}\right) \psi^{\prime}+\frac{\chi^{2}}{f^{2}} \psi-\frac{m^{2}}{f} \psi=0 .
\end{aligned}
$$


Nótese que el sistema de arriba es precisamente el superconductor holográfico $U(1)$ original estudiado por primera vez en [27]. Para asegurarnos quela norma de la corriente en el horizonte sea finita, tenemos que pedir que el campo escalar se regular mientras que el campo de gauge tiene que anularse $\chi(\rho=1)=0$. Con estas condiciones de borde, el comportamiento asintótico de so campso en el borde conforme es

$$
\begin{gathered}
\chi=\bar{\mu}-\frac{\bar{n}}{\rho}+O\left(\frac{1}{\rho^{2}}\right), \\
\psi=\frac{\psi_{1}}{\rho}+\frac{\psi_{2}}{\rho^{2}}+O\left(\frac{1}{\rho^{3}}\right) .
\end{gathered}
$$

Para el valor elegido de masa del escalar, los dos términos en la expansión del escalar son normalizables [117]. Considerando que uno o el otro sea el valor de espectación de la teoría del borde conduce a dos teorías diferentes. En lo que sigue vamos a considerar el caso en el que $\psi_{1}$ es interpetado como la fuente y $\psi_{2}$ como el vev de un operador de dimensión de masa dos.

Los parámetros adimensionales están relacionados con las cantidades físicas a través de

$$
\begin{aligned}
\bar{\mu} & =\frac{3}{4 \pi T} \mu, \\
\bar{n} & =\frac{9}{16 \pi^{2} T^{2} L^{2}} n, \\
\psi_{1} & =\frac{3}{4 \pi T L^{2}} J_{\mathcal{O}}, \\
\psi_{2} & =\frac{9}{16 \pi^{2} T^{2} L^{4}}\langle\mathcal{O}\rangle,
\end{aligned}
$$

donde $\mu, n$ y $J_{\mathcal{O}},\langle\mathcal{O}\rangle$ son el potencial químico, la densidad de carga y las fuentes y vev del operador $\mathcal{O}$ de dimensión 2 , respectivamente. A partir de ahora vamos a elegir $L=1$. En lo que sigue vamos a trabajar en el ensemble gran canónico. En práctica variamos el parámetro adimensional $\bar{\mu}$. Debido a al simetría conforme subyacente esto puede se pensado o bien como fijar el potencial químico $\mu$ y variar la temperatura $T$ o como variar al temperatura y fijar el potencial químico. Definimos la temperatura como $T / T_{c}=\bar{\mu}_{c} / \bar{\mu}$ y fijamos $\mu=1$.

La rotura espontánea de simetría se produce a temperaturas bajas o potenciales químicos grandes. Esto gatilla un valor de espectación no nulo para el campo escalar sin prender la fuente $J_{\mathcal{O}}$. Para $\bar{\mu}$ pequños el campo escalar es nuloy la ecuaciones de movimiento son resueltas por $\chi=\bar{\mu}(1-1 / \rho)$ y $\psi=0$. El sistema está entonces en la fase simétrica. Sin embargo, al bajar la temperatura el sistema se vuelve inestabel frente a la condensación de un escalar [26, 27]. En [100] se muestra que a la temperatura crítica el QNM más bajo del campo escalar se vuelve inestable, i. e. cruza al semiplano superior.

La densidad de energía libre viene dada por la acción on-shell renormalizada,

$$
F=-T S_{r e n}=-T\left(\frac{1}{2} \mu n-\int_{r_{H}}^{\infty} d r \frac{r^{2} \psi^{2} \chi^{2}}{f}\right) .
$$

En la figura 4.2 las energías libres para las fases simétrica y rota son comparadas. Es claro que para $T<T_{c}$ la solución con condensado es siempre preferida y entonces el sistema sufre una transición continua de segundo orden a una fase superconductora. Nótese que la 

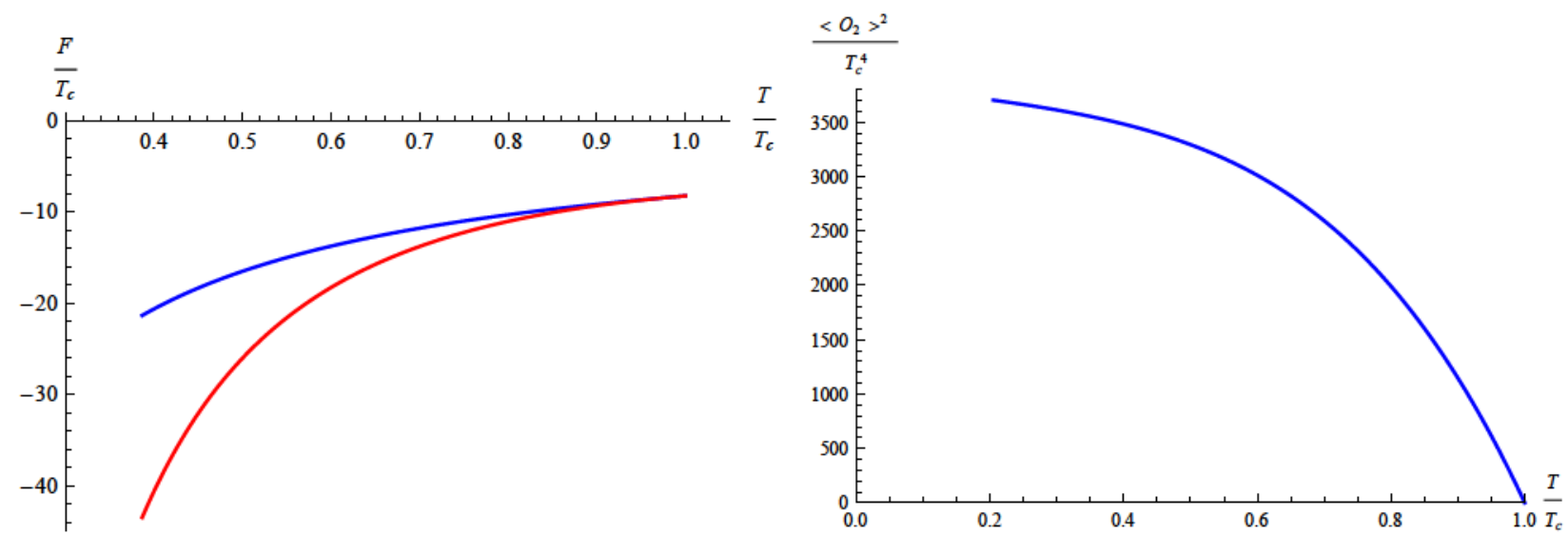

Figura 4.2: (Izquierda) la enegía libre de las soluciones de background trivial (azul) y con condensado (rojo) a temperaturas bajas, $T<T_{c}$. (Derecha) valor del condensado en el ensemble grna canónico como función de $T / T_{c}$.

presencia del segundo escalar no juega ningún rol en la estructura del diagrama de fases. Esto se debe a que siempre pedimos que se anule, $\lambda=0$.

Con el fin de sacar el espectro de QNMs, prendemos las fluctuaciones de los campos de background

$$
\begin{aligned}
\Psi^{\mathrm{T}} & =(\eta(\rho, t, x), \psi(\rho)+\sigma(\rho, t, x)) \\
A & =\left(\chi(\rho)+a_{t}(\rho, t, x)\right) d t+a_{x}(\rho, t, x) d x .
\end{aligned}
$$

No incluimos las fluctuaciones transversas ya que se desacoplan de las propiedades físicas interesantes del presente modelo.

En la fase normal, i.e. expandiendo alrededor de $\psi(\rho)=0$, el sistema se reducel al del superconductor holográfico $U(1)$ estudiado en [100] con dos copias de las fluctuaciones escalares,

$$
\begin{aligned}
f s^{\prime \prime}+s^{\prime}\left(f^{\prime}+\frac{2 f}{\rho}\right)+\left(\frac{(\chi+\omega)^{2}}{f}-\frac{k^{2}}{\rho^{2}}-m^{2}\right) s & =0, \\
f a_{t}^{\prime \prime}+\frac{2 f}{\rho} a_{t}^{\prime}-\frac{k^{2}}{\rho^{2}} a_{t}-\frac{\omega k}{\rho^{2}} a_{x} & =0, \\
f a_{x}^{\prime \prime}+f^{\prime} a_{x}^{\prime}+\frac{\omega^{2}}{f} a_{x}+\frac{\omega k}{f} a_{t} & =0, \\
\frac{i \omega}{f} a_{t}^{\prime}+\frac{i k}{\rho^{2}} a_{x}^{\prime} & =0,
\end{aligned}
$$

donde $s$ es tanto la fluctuación $\eta$ como $\sigma$. La ecuación para el modo complejo conjugado $\bar{s}$ puede ser obtenida cambiando el sifno del potencial $\chi$ en (4.30). La frecuencia y momento asociados a los físicos son

$$
\begin{aligned}
\omega & =\frac{3}{4 \pi T} \omega_{p h}, \\
k & =\frac{3}{4 \pi T} k_{p h} .
\end{aligned}
$$


Las fluctuaciones para el escalar y el campo de gauge se desacoplan por completo en la fase normal. Esta es una consecuencia de trabajar en el límite de prueba. El espectro de QNMs del campo $U(1)$ en la fase normal es simplemente aquel del un campo electromagnético en un background AdS-Sch. Las fluctuaciones longitudinales contienen un modo hidrodinámico, $\omega=-i D k^{2}$, reflejando el carácter difusivo de fluidos normales. En unidades físicas $D=$ $3 /(4 \pi T)$. Debido a la falta de un tensor de energía momento para la teoría de campos del borde en el límite de prueba, el polo difusivo es el único modo hidrodinámico de la fase normal.

Hay dos copias de las fluctuaciones escalares. Los modos quasinormales de $\eta$ y $\sigma$ se mueven para el origen a medida que bajamos la temperatura, mientras que los modos para $\bar{\eta}$ y $\bar{\sigma}$ tiene masas más grandes para temperaturas más bajas. A medida que nos acercamos a la temperatura crítica $T=T_{c}$, los QNMs más bajos de $\eta$ y de $\sigma$ se hacen no masivos, disparando la transición de fase: el campo escalar adquiere un valor de espectación (vev) no trivial contal de evitar que sus fluctuaciones se vuelvan taquiónicas. Por simetría podemos elegir el condensado de manera tal que resita complentamente en el campo $\psi$. La flucutación $\sigma$ se acopla entonces a las fluctuaciones edl campo de gauge tal como en [100]. Entonces el espectro de QNM contiene un modo de Goldstone con relación de dispersión lineal $\omega= \pm v_{s} k+O\left(k^{2}\right)$. Esto es lo usual en bosones de Goldstone del tipo I asociados a la rotura de la simetría de gauge $U(1)$. Como fue mostrado en [100] puede ser interpretado como el modo de sonido del superfluido dual en la fase superconductora. Que pasa entonces a los QNMs en las fluctuaciones del segundo escalar $\eta$ ? A la temperatura crítica hay también un modo no masivo en este sector ya que su espectro de QNM es simplemente una copia del primero. Como no hay operadores generando la simetría $S U(2)$ en la teoría de campos dual, argumentos estándar acerca de la aparición de modos de Goldstone a priori no aplicarían. Tres posibilidades lógicas emergen entonces: El modo se vuelve inestable para $T<T_{c}$, se puede volver masivo o quedar no masivo, haciendo el papel de un bosón de Goldstone inesperado para la simetría global del bulk $S U(2)$. Pronto veremos que la última posibilidad es la que ocurre y que la relación de dispersión de $\eta$ va a corresponderse con un bosón de Goldstone de tipo II con una relación de dispersión cuadrática, $\omega \propto k^{2}$.

En la fase rota, las ecuaciones de movimiento son

$$
\begin{aligned}
& 0=f \eta^{\prime \prime}+\eta^{\prime}\left(f^{\prime}+\frac{2 f}{\rho}\right)+\left(\frac{(\chi+\omega)^{2}}{f}-\frac{k^{2}}{\rho^{2}}-m^{2}\right) \eta \\
& 0=f \delta^{\prime \prime}+\delta^{\prime}\left(f^{\prime}+\frac{2 f}{\rho}\right)+\left(\frac{\chi^{2}}{f}+\frac{\omega^{2}}{f}-\frac{k^{2}}{\rho^{2}}-m^{2}\right) \delta-\frac{2 i \omega \chi}{f} \zeta-i \psi\left(\frac{\omega}{f} a_{t}+\frac{k}{\rho^{2}} a_{x}\right) \\
& 0=f \zeta^{\prime \prime}+\zeta^{\prime}\left(f^{\prime}+\frac{2 f}{r}\right)+\left(\frac{\chi^{2}}{f}+\frac{\omega^{2}}{f}-\frac{k^{2} L^{2}}{r^{2}}-m^{2}\right) \zeta+\frac{2 i \omega \chi}{f} \delta+\frac{2 \chi \psi}{f} a_{t} \\
& 0=f a_{t}^{\prime \prime}+\frac{2 f}{\rho} a_{t}^{\prime}-\left(\frac{k^{2}}{\rho^{2}}+2 \psi^{2}\right) a_{t}-\frac{\omega k}{\rho^{2}} a_{x}-2 i \omega \psi \delta-4 \chi \psi \zeta \\
& 0=f a_{x}^{\prime \prime}+f^{\prime} a_{x}^{\prime}+\left(\frac{\omega^{2}}{f}-2 \psi\right) a_{x}+\frac{\omega k}{f} a_{t}+2 i k \psi \delta \\
& 0=\frac{i \omega}{f} a_{t}^{\prime}+\frac{i k}{\rho^{2}} a_{x}^{\prime}+2 \psi^{\prime} \delta-2 \psi \delta^{\prime}
\end{aligned}
$$

donde hemos dividido $\sigma=\zeta+i \delta$ en parte real e imaginaria. El sistema (4.37)-(4.41) es 
nuevamente aquel estudiado en [100]. Este sector, que también aparece en el modelo gaugeado que será presentado luego, se desacopla de la fluctuación en $\eta$. Nótese que aun cuando pa ecuación (4.36) es formalmente igual a la de la fase normal, el background $\chi$ llevando a características no triviales en el sector $\eta$ tales como la presencia de exitaciones no masivas.
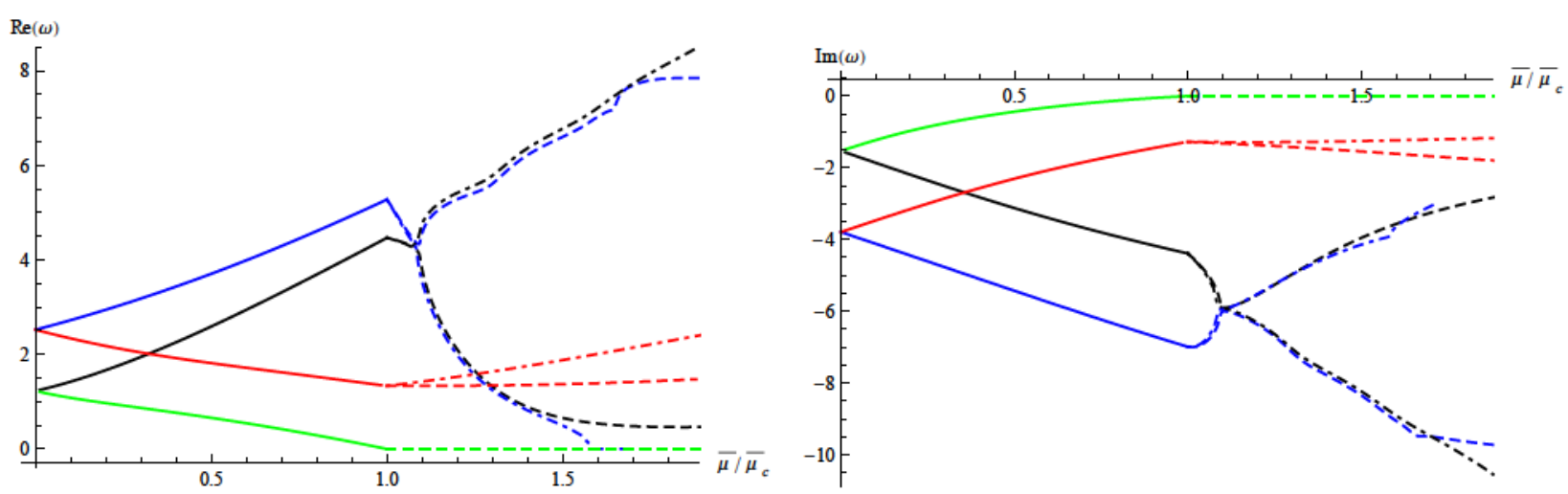

Figura 4.3: Partes real (izquierda) e imaginara (derecha) de los QNMs escalares más bajos como función del potencial químico. Las líneas sólidas corresponden a la fase normal. Para la fase rota líneas dashed representan los modos para el escalar adicional mientras que líneas con puntos representan los modos del superconductor holográfico $U(1)$ común.

La figura 4.3 muestra el espectro de excitaciones quasinormales del doblete escalar. En la fase normal tenemos dos copias degeneradas del espectro que se desdoblan parcialmente luego de la transición de fases. Es claro que las dos exitaciones mñas bajas se hacen no masivas al potencial químico crítico y que luego permanecen no masivas en la fase supercondutora. Éstas pueden ser identificadas con los bosones de Goldstone en la fase rota. El resto de las exitaciones permanecen gapeadas en la fase rota. Nótese que la primer exitación $\bar{\eta}$ (línea entrecortada negra de la figura 4.3) no sigue el comportamiento universal esperado en la fase rota, i.e. no es linear en $\mu$. Este modo es equivalente al modo especial gapeado $\omega_{4}$ en el modelo de teoría de campos de la sección 4.2. Sin embargo, ya fue mencionado que el modelo ungauged no satisface toso los teoremas sobre rotura de simetría y entonces desviaciones del comportamiento universal no deberían de sorprendernos. El comportamiento de los modos con gap es de hecho similar a auqel de los modos del modelo $U(1)$. En la fase no rota, podemos distinguir los modos que vienen de fluctuaciones tipo $s$ de aquellas fluctuaciones que vienen de su complejo conjugado $\bar{s}$. El primero se hace más liviano mientras el segundo más pesado ${ }^{7}$. En la fase rota es más útil usar partes real e imaginaria, al menos para el escalar que se mezcla con las fluctuaciones de gauge, i.e. la componente de abajo del doblete en nuestra convención. Entocens a priori no podemos hablar de fluctuaciones tipo $s$ y $\bar{s}$. Pero toavía podemos estudiar a que soluciones los modos tipo $s$ y $\bar{s}$ se conectan en la fase rota. Acá vemos un patrón interesante: los modos tipo $s$ se separan en la fase rota mientras que los tipo $\bar{s}$ permaneces casi degenerados cerca de la transición de fase (al menos a momento cero). Esto es sorprendente dado que son soluciones de sistemas completamente distintos, uno viene de una única ecuación diferencial mientras el otro viene de un sistema complicado de ecuaciones acopladas. Sin embargo, para temperaturas pequeñas se separan y de hecho la parte real del más bajo modo del sector $U(1)$ se va a cero a temperatura finita. Para

\footnotetext{
${ }^{7}$ Este comportamiento se revertiría si tomásemos el potencial químico negativo.
} 
temperaturas por debajo de $T \approx 0,63 T_{c}$ se convierte en un modo puramente imaginario.

\subsubsection{Modo de sonido}

Hay dos modos no masivos en la fase rota. El primero es un bosón de Goldstone tipo I que aparece debido a la rotura de la simetría de gauge $U(1)$. En [100], se muestra que este modo corresponde al modo de sonido del superfluido dual y que en el límite hidrodinámico tiene una relación de dispresión lineal

$$
\omega_{I}= \pm\left(v_{s} k+\bar{b} k^{2}\right)-i \Gamma_{s} k^{2}
$$

donde $v_{s}$ es la velocidad del sonido y $\Gamma_{s}$ es su atenuación. Resulta que la parte real de la relación de dispersión también tiene una componente real. Esta componente es muy pequeña y despreciable comparada con el término lineal que determina la velocidad del sonido. En [100] esta parte real cuadrática no fue estudiada.
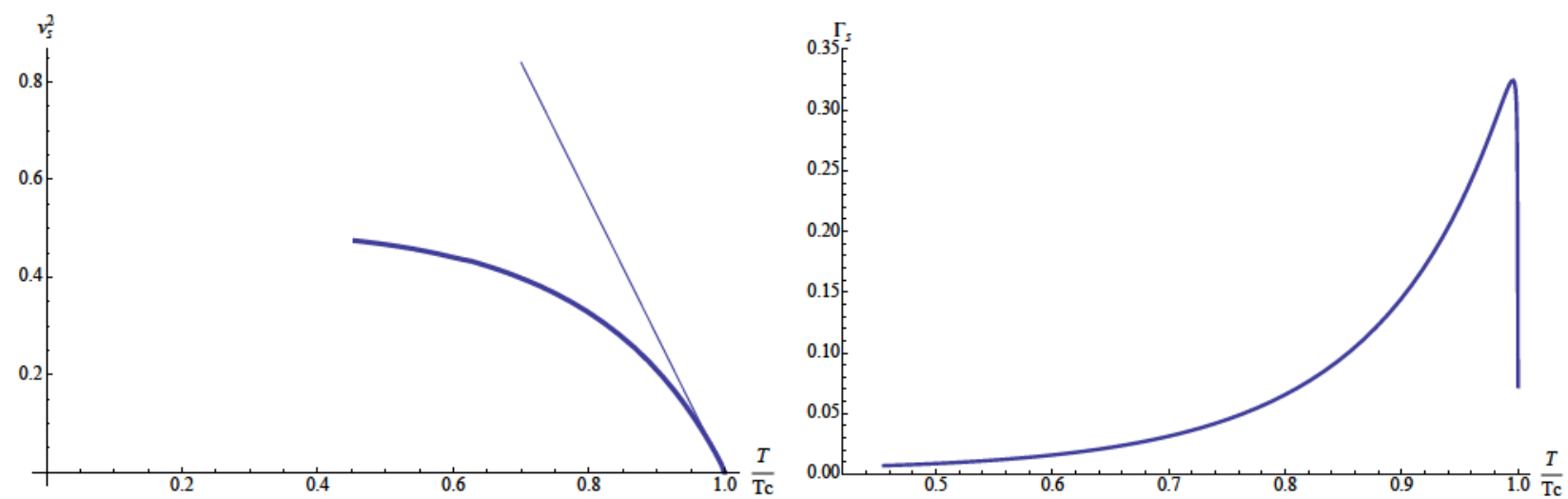

Figura 4.4: Velocidad de sonido y amortiguamiento para el modelo $U(1)$. La velocidad del sonido va a cero en la temperatura crítica. La constante de decaimeiento primero crece rápidamente y luego cae monótonamente. Precisamente a la temperatura crítica, su valor es tal que el modo de sonido se conecta de manera continua con el el modo escalar que se hace no masivo entonces. El pico en la constante de amortiguacion se ubica cerca de la temperatura crítica y no fue resuelto en [100].

Para temperaturas muy pequeñas la velocidad se aproxima a su valor conforme $v_{s}^{2}=1 / 2$ wientras que el ancho va a cero, ver la figura 4.4. Cerca de la transición de fase, la velocidad del sonido tiene un comportamiento de campo medio ya que como función de la temperatura

$$
v_{s}^{2} \approx 2,8\left(1-\frac{T}{T_{c}}\right)
$$

Como es esperado, a $T=T_{c}$ la velocidad del sonido se anula. Este hecho puede ser asociado a que en la transición de fase la masa $m_{*}^{2}=M^{2}-\mu^{2}$ satisface $m_{*}^{2}=v^{2}=0$, como es de esperarse, y entonces el campo escalar complejo, cargado bajo una simetría $U(1)$ se hace no masivo.

De hecho, uno puede escribir la acción efectiva, análoga a (4.4), para un campo complejo con masa $M$, en presencia de un potencial químico para una simetría $U(1)$ que fue rota 
espontáneamente. Las exitaciones encima del background que rompe $U(1)$ tienen una relación de dispersión igual a (4.8)-(4.9), siendo (4.8) los bosones de Goldstone de tipo I. Como es una característica general de estos modelos sigma lineales que el coeficiente en frente del término lineal en momento depende de $m_{*}^{2}$, como fue explicitamente chequeado en este caso (ver (4.8)). Entonces, en la transición de fases el término dominante en la relación de dipersión es $O\left(k^{2}\right)$; este efecto puede ser visto claramente con nuestros métodos numéricos, como se ve en la figura 4.5. Como el espectro de QNM tiene que varia de manera continua a lo largo de una transición de fase de segundo orden las partes real e imaginaria del término $k^{2}$ tiene que coincidir a $T=T_{c}$ con las que fueron obtenidas estudiando los escalares no masivos en la fase normal. Numéricamente encontramos $\bar{b}\left(T_{c}\right)=0,22$ y $\Gamma_{s}\left(T_{c}\right)=0,071$.
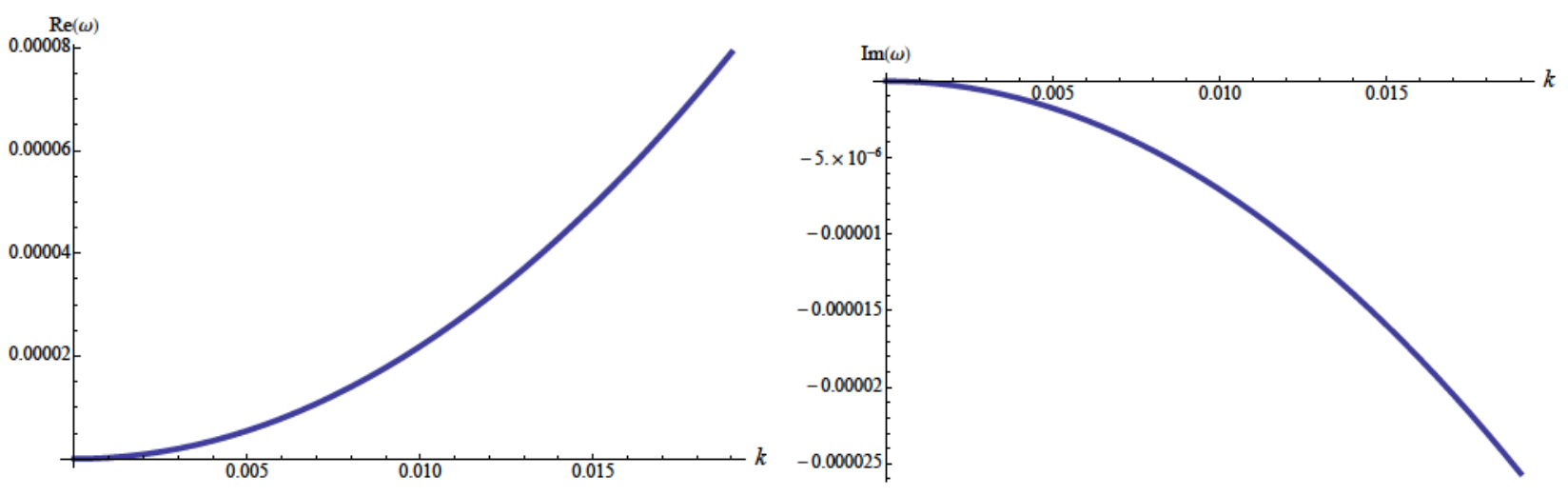

Figura 4.5: Relaciones de dipersión de $\operatorname{Re} \omega$ (izquierda) y $\operatorname{Im} \omega$ (derecha) a $T=T_{c}$ para los bosones de Goldstone tipo I en el sistema estudiado en [100]. El comportamiento Re $\omega \sim k$ se hace cuadrático justo a esta temperatura: $\operatorname{Re} \omega=\bar{b} k^{2}$. El coeficiente es $\bar{b}=0,22$, que resulta pegarse con el resulta que aparece cuando uno se acerca a $T_{c}$ desde arriba (i.e. desde la fase no rota).

\subsubsection{Modo pseudodifusivo}

En la fase no rota este modelo tiene solo un modo hidrodinámico, el modo difusivo $\omega=-i D k^{2}+O\left(k^{4}\right)$ con $D=3 /(4 \pi T)$ en unidades físicas. Los modos de sonido normal y de shear tienen su origen en las fluctuaciones de la métrica y por ese motivo están ausentes en el límite de desacomplamiento que estamos estudiando. La transición de fase a la fase rota es de segundo orden. Para el espectro de modos cuasinormales esto implica que los mismos se ocnectadn de manera continua a través de la transición de fase. En el caso del modo difusivo debe existir un modo no masivo puramente imaginario. La hidrodinámica implica sin embargo que los únicos modos no masivos sean los Goldstone. No demasiado lejos de la transición de fase, i.e. para $T \lesssim T_{c}$ el modo difusivo en la fase rota debe evolucionar a un modo con relación de dispersión

$$
\omega=-i \gamma(T)-i D(T) k^{2}+O\left(k^{4}\right),
$$

como se muestra en la figura 4.6.

Debemos decir que el modo difusivo desarrolla un ga en la fase rota y se transforma en lo que ha sido llamado un modo pseudodifusivo en [100]. Precisamente a momento $k=0$ Este 
modo masivo pseudodifusivo explica los efectos observados en [102] sobre la respuesta tardía en superconductores holográficos. Para temperaturas $T \lesssim T_{c}$ el modo pseudodifusivo es el modo que queda más cerca al eje real y por ello domina la respuesta a tiempos largos de cualquier perturbación, como por ejemplo los quenches estudiados en [102]. Se sigue que el condensado muestra un decaimeinto puramente exponencial ya que este modo no tiene una frecuencia real. La existencia de este modo puede ser rastreada hasta el modo difusivo de la fase normal. Esperamos entonces que sea una característica propia de una gran clase de superfluidos (incluso superfluidos no holográficos).
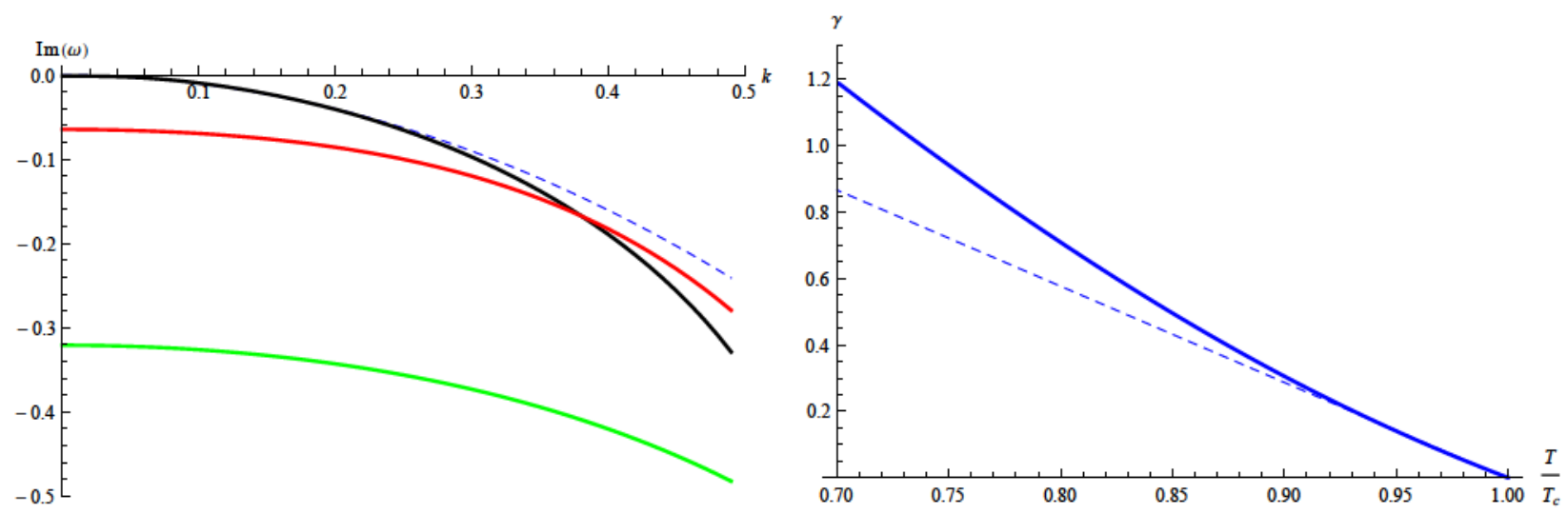

Figura 4.6: (Izquierda) relación de dispersión de un modo pseudodifusivo gapeado en la fase rota para tres temperaturas diferentes. El gap se ensancha a medida que la temperatura baja. (Derecha) Gap $\gamma$ como función de la temperatura reducida $T / T_{c}$. A media que uno se acerca a la temperatura crítica desde abajo el gap desaparece.

El gap $\gamma$ crece a medida que la temperatura decrece. Por otro lado, hay QNMs (conectados a los QNM de los escalares en la fase no rota) cuya parte imaginaria apenas depende de la temperatura. A cierta temperatura de crossover $T_{*}$ el gap del modo pseudodifusivo es más grande que la parte imaginaria de esots modos, domo se muestra en la figura 4.7. Entonces el patrón de respuesta cambia de un decaimiento puramente exponencial a una oscilación exponencialmente suprimida. Numericamente ecnontramos que la temperatura de crossover es $T_{*}=0,69 T_{c}{ }^{8}$ Estos cambios en la respuesta a tiempos grandes de tipo crossover aparecen frecuentemente en el detalle de espectros de QNMs de teorías holográficas, [118, 119, 120]. De hecho, este decaimiento puramente exponencial aplica no sólo al parámetro de orden, si no que a todos los operaroes que corresponden a campo que participan en el sietemde fluctuaciones (4.37)-(4.41), e.g. la densidad de carga o la compenete $x$ de la corriente.

Para momento finito el patrón de respuesta es esperable que sea distinto. Ahora uno debería tener en cuenta también el modo de sonido. Mientras que a momento exactamente nulo el modo de sonido, i.e. el modo de Goldstone, se degenera a un cambio de fase constante para el condensado, a momento pequeño pero distinto de cero la respuesta a tiempos largos debería estar dominada por las frecuencias complejas (4.42). Sin embargo, si uno mira la respuesta del parámetro de orden invariante $|\mathcal{O}|$ los modos de Goldstone, siendo rotaciones locales de la fase, pueden ser proyectados a cero.

\footnotetext{
${ }^{8}$ Esta es más baja que en el modelo [102]. La diferencia se debe probablemente al hecho de que trabajamos en el límite de desacoplamiento.
} 

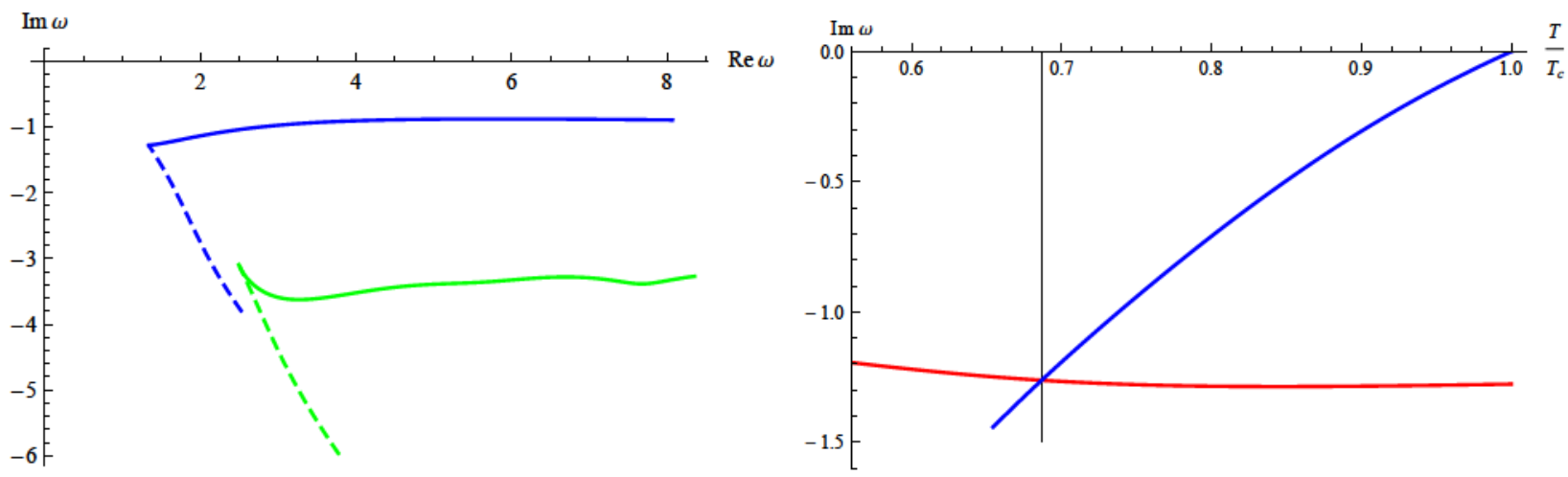

Figura 4.7: (Left) Continuation of the second and third scalar QNM into the broken phase. The real part grows as the temperature is lowered whereas the imaginary part shows very little dependence on $T$. (Right) The gap $\gamma$ (blue line) and the imaginary part of the lowest (scalar) mode fluctuation (red line) in the broken phase are shown as function of $T / T_{c}$. At $T_{*} \approx 0,69 T_{c}$ the imaginary parts cross. For lower temperatures the late time response is not dominated anymore by the pseudo diffusion mode and consequently is in form of a exponentially decaying oscillation.

\subsubsection{Modos de Goldstone tipo II}

El segundo modo no masivo es le bosón de Goldstone asociado con romepr la simetría $S U(2)$ global del bulk. Puede ser ajustado a una relación de dispersión cuadrática

$$
\omega_{I I}= \pm b k^{2}-i c k^{2}+O\left(k^{4}\right),
$$

en el límite de longitudes de onda grandes. Entonces tiene las caracterísitcas de un bosón de Goldstone tipo II. En la figura 4.8 la relación de dispersión para el modo no masivo de $\eta$ es mostrada para varias temperaturas así como sus ajustes en el límite hidrodinámico. Es claro que hay un buen acuerdo en el régimen de validez del límite de bajas energías.
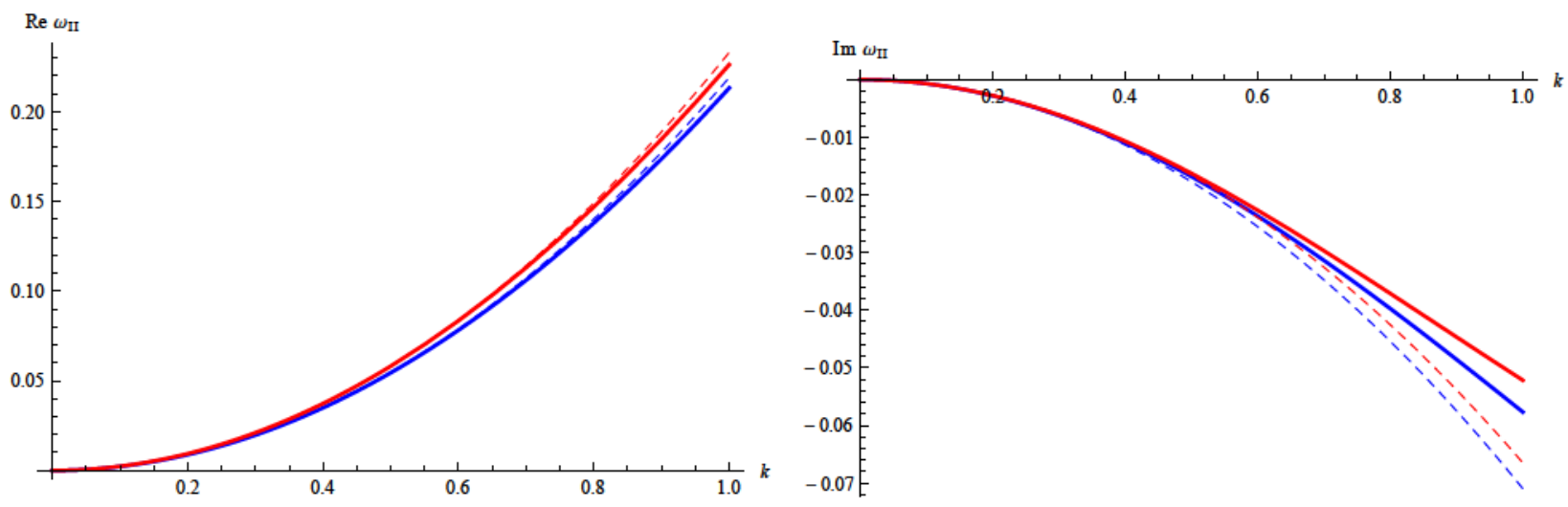

Figura 4.8: Partes real (izquierda) e imaginaria (derecha) del bosón de GOldstone tipo II como función del momento a $T / T_{c}=0,9998$ (azul) y $T / T_{c}=0,704$ (rojo) . Las líneas continuas corresponden a la data numérica mientras que las líneas entrecortadas corresponden a un ajuste cuadrático a la relación de dispersión $\omega_{I I}=b k^{2}-i c k^{2}$.

Los coeficientes en la relación de dispersión hidrodinámica (4.45) como función de la temperatura se muestran en la figura 4.9. Cerca de la transición de fase tienen una relación 
lineal con la temperatura reducida

$$
\begin{aligned}
& b(T)=0,22+0,049\left(1-\frac{T}{T_{c}}\right), \\
& c(T)=0,071-0,0014\left(1-\frac{T}{T_{c}}\right) \text { near } T_{c} .
\end{aligned}
$$

Nótese que en la transición de fase el modo de sonido y el Goldstone tipo II se deben comportar de la misma manera debido a la continuidad de los modos a lo largo de la transición de fase y a que son degenerados en la afase normal. De hecho, en la transición los valores $b=\bar{b}=0,22$ y $c=\Gamma_{s}=0,071$, values that of course coincide with those of the lowest scalar mode in the normal phase coinciden con aquellos del escalar en la fase normal. Por otro lado, es interesante notar que en la fase rota el compotamiento de los coeficientes de los modos Godstone tipo II es completamente diferente a aquel del modo sonoro del superfluido. A diferencia de la velocidad del sonido, que se anula en la transición de fase, el coeficiente $b$ del modo de Goldstone tipo II tiene un valor finito en la transición de fase. Este resultado persiste para el modelo gaugeado. La atenuacion por otro lado, como ocurre en el sector $U(1)$, tiene un valor finito en la transición de fase y luego decrece con la temperatura, reflejando el hecho de que el fluido es mas ideal a medida que bajamos la temperatura.
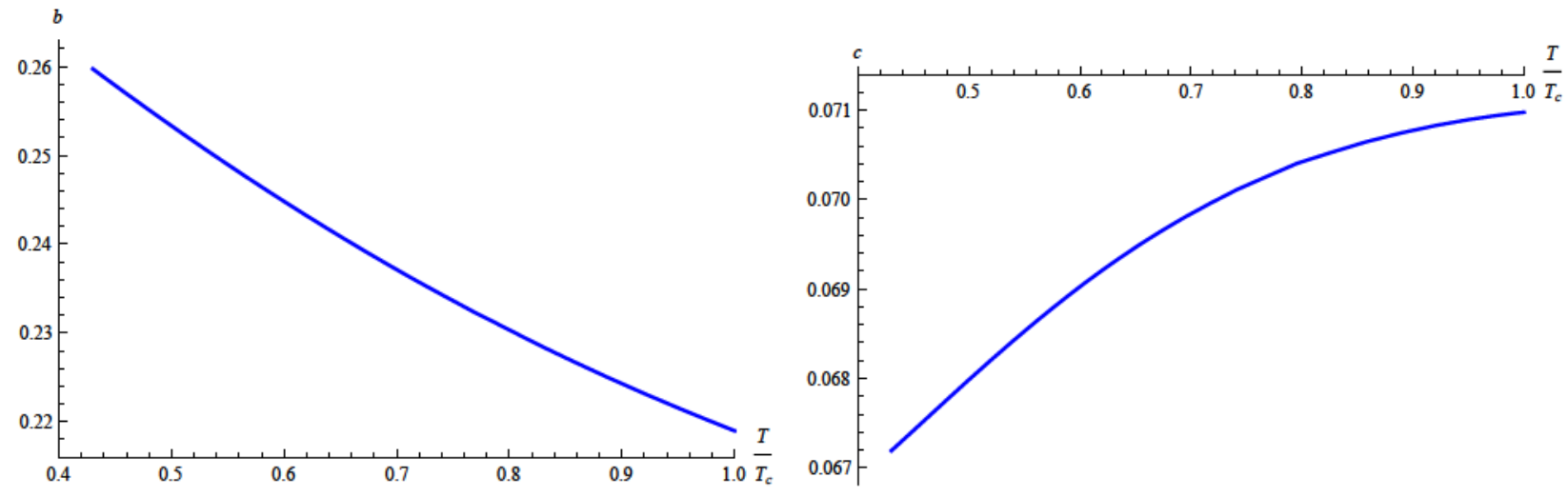

Figura 4.9: Coeficientes de la relación de dispersión del modo de Goldstone tipo II $\omega_{I I}=$ $b k^{2}-i c k^{2}$, como función de la temperatura. La dependencia con la tempertatura es archi suave.

\subsection{El modelo gaugeado}

Discutamos ahora el modelo completamente gaugeado. Consideremos el siguiente lagrangiano para un campo escalar complejo viviendo en la representación fundamental de la simetría de gauge $U(2)$ local en el bulk,

$$
S=\int \sqrt{-g} \mathcal{L}=\int d^{4} x \sqrt{-g}\left(-\frac{1}{4} F^{\mu \nu c} F_{\mu \nu}^{c}-m^{2} \Psi^{\dagger} \Psi-\left(D^{\mu} \Psi\right)^{\dagger} D_{\mu} \Psi\right),
$$

donde

$$
\Psi=\left(\begin{array}{l}
\lambda \\
\psi
\end{array}\right), \quad A_{\mu}=A_{\mu}^{c} T_{c}, \quad D_{\mu}=\partial_{\mu}-i A_{\mu}
$$


y $c=0,1,2,3$ es el índice de color. El campo $\Psi$ juega el papel del condensado. El valor de espectación de su operador dual dispara la rotura espontánea de la simetría $U(2)$ global de la teoría del borde. Por simplicidad, elegimos que $\lambda=0$ en el background. $T_{c}$ son los generadores de $U(2)$ :

$$
\begin{aligned}
T_{0}=\frac{1}{2} \mathbb{I}, \quad T_{i} & =\frac{1}{2} \sigma_{i}, \\
\left\{T_{i}, T_{j}\right\}=\frac{1}{2} \delta_{i j} \mathbb{I}, \quad\left\{T_{0}, T_{i}\right\} & =\frac{1}{2} \sigma_{i} .
\end{aligned}
$$

Nótese que estamos trabajando en el límite de prueba, así que la métrica de background la elegimos como Schwarzschild-AdS black brane de (4.16). Pr otro lado, el campo de gauge ahora es

$$
A_{0}^{(0)} \equiv \Phi(r), \quad A_{0}^{(3)} \equiv \Theta(r)
$$

Siendo todas las demás componentes del campo de gauge nulas. Como en la sección anterior, vamos a usar coordenadas adimensionales definidas por el escaleo dado en (4.17).

Las ecuaciones de movimiento para este ansatz

$$
\begin{aligned}
\psi^{\prime \prime}+\left(\frac{f^{\prime}}{f}+\frac{2}{\rho}\right) \psi^{\prime}+\frac{(\Phi-\Theta)^{2}}{4 f^{2}} \psi-\frac{m^{2}}{f} \psi & =0, \\
\Phi^{\prime \prime}+\frac{2}{\rho} \Phi^{\prime}-\frac{\psi^{2}}{2 f}(\Phi-\Theta) & =0, \\
\Theta^{\prime \prime}+\frac{2}{\rho} \Theta^{\prime}+\frac{\psi^{2}}{2 f}(\Phi-\Theta) & =0 .
\end{aligned}
$$

Nótese que de (5.8) se sigue que no podemos simplemente prender $\Phi$ sin tampoco prender un $\Theta$ no trivial. Por su poio estamos solamente intereados en prender un potencial químico en el overall $U(1)$, y por este motivo vamos a imponer $\Theta(\rho \rightarrow \infty)=0$ y permitir un valor de borde finito para $\Phi$.

El sistema de ecucaciones acoplado de arriba puede ser simplificado redefiniendo $\chi \equiv$ $\frac{1}{2}(\Phi-\Theta)$ y $\xi \equiv \frac{1}{2}(\Phi+\Theta)$. Usando (5.7) y (5.8), vemos que las ecuaciones resultantes para estos campos es $^{9}$

$$
\begin{aligned}
\Psi^{\prime \prime}+\left(\frac{f^{\prime}}{f}+\frac{2}{\rho}\right) \Psi^{\prime}+\frac{\chi^{2}}{f^{2}} \Psi-\frac{m^{2}}{f} \Psi & =0, \\
\chi^{\prime \prime}+\frac{2}{\rho} \chi^{\prime}-\frac{2 \Psi^{2}}{f} \chi & =0, \\
\xi^{\prime \prime}+\frac{2}{\rho} \xi^{\prime} & =0,
\end{aligned}
$$

donde hemos redefinido $\psi \rightarrow \sqrt{2} \Psi$. Como siempre, elegimos las condiciones de borde $\chi(\rho=$ $1)=0, \xi(\rho=1)=0$ así como regularidad para $\Psi$. Tener una teoría de campos dual con sólo un potencial químico encendido, implica que $\chi$ y $\xi$ deben tomar el mismo valor no trivial en el borde para asegurar que $\Theta$ se anule asintóticamente. Nótese que $\xi$ se desacopla

\footnotetext{
${ }^{9}$ Estas ecuaciones de movimiento corresponden al probe limit de las estudiadas en [121] como el dual de superconductores desbalanceados. Nótese sin embargo que en [121] la simetría de gauge era $U(1) \times U(1)$ en lugar de $U(2)$ como es el caso de este trabajo.
} 
por completo. El sistema restante (4.54)-(5.9) es nuevamente el background encontrado para el superconductor holográfico s-wave $U(1)$ ampliamente estudiado. Entonces, el background para el modelo gaugead $U(2)$ contiene al superconductor abeliano más un sector desacoplado $U(1)$.

El campo $\chi$ yace en la dirección de uno de los generadores rotos, que es la combinación lineal $\frac{1}{2}\left(T_{3}-T_{0}\right)$, mientras $\xi$ yace en la dirección del $U(1)$ conservado dado por $\frac{1}{2}\left(T_{3}+T_{0}\right)$.

La expansión asintótica para los campos cerca del borde conforme es

$$
\begin{gathered}
\chi=\bar{\mu}_{\chi}-\frac{\bar{n}_{\chi}}{\rho}+O\left(\frac{1}{\rho^{2}}\right), \\
\xi=\bar{\mu}_{\xi}-\frac{\bar{n}_{\xi}}{\rho}+O\left(\frac{1}{\rho^{2}}\right), \\
\Psi=\frac{\psi_{1}}{\rho}+\frac{\psi_{2}}{\rho^{2}}+O\left(\frac{1}{\rho^{3}}\right) .
\end{gathered}
$$

El mapeor de los coeficientes en las ecuaciones anteriores a las condiciones de borde es $\bar{\mu}_{\chi}=\bar{\mu}_{\xi}=\bar{\mu}$. Una vez más, vamos a concentrarnos sólo en la teoría $\mathcal{O}_{2}$ de manera exclusiva, y por eso vamos a exigir $\psi_{1}=0$.

Las ecuaciones (4.54)-(5.9) permiten escuaciones con un condensado no nulo, lo que implica que $\frac{1}{2}\left(T_{3}-T_{0}\right)$ va a estar roto de manera espontánea. esta solución debe ser encontrada de manera numérica, ya que el sistema es no lineal. Sin embargo, (4.56) sí tiene una solución analítica

$$
\xi=\bar{\mu}\left(1-\frac{1}{\rho}\right)
$$

y entonces $\bar{n}_{\xi}=\bar{\mu}$.

Cuando la simpetía no está rota, $\Psi=0$, la ecuación para $\chi$ tiene por supuesto

$$
\chi=\bar{\mu}\left(1-\frac{1}{\rho}\right)
$$

como solución también. Entonces, en la fase no rota

$$
\begin{aligned}
& \Theta=0, \\
& \Phi=2 \bar{\mu}\left(1-\frac{1}{\rho}\right) .
\end{aligned}
$$

Este comportamiento refleja el hecho de que $T_{3}$ es completamente independiente de $T_{0}$ en la fase no rota. Sin embargo, una vez que prendemos el condensado, el juego entre $T_{3}$ y $T_{0}$ (recuérdese que la simetría restante es una combinación de ambos) hace imposible fijar sólo uno de estos campos a cero.

Finalmente, mencionemos que con el fin de relacionar los parámetros adimensionales con los físicos, necesitamos aplicar el mismo diccionario (4.23)-(4.26) usado para el modelo ungauged.

\subsubsection{Densidad de carga en la fase rota}

De acuerdo con $[104,109]$ podemos esperar la presencia de modos de Goldstone tipo II si los generadores de la simetría rota satisfacen

$$
\left\langle\left[Q_{a}, Q_{b}\right]\right\rangle=B_{a b}
$$


con al menos un $B_{a b} \neq 0$. En nuestro caso tenemos $\left[Q_{1}, Q_{2}\right]=i Q_{3}$. Por lo tanto en nuestra fase rota estamos interesados en un valor de espectación no nulo para la densidad de carga $\left\langle Q_{3}\right\rangle=n_{\Theta}$. Como hemos argumentado antes, en la fase no rota tenemos necesariamente $\Theta(r)=0$. Esto pasa ya que $\chi$ y $\xi$ obedecen la misma ecuación diferencial y las constantes de integración tienen que ser iguales en si queremos evitar prender una fuente para $\Theta$. Ahora queremos encontrar si hay o no un valor de espectación para $\Theta$ generado espontáneamente en la fase rota.

El campo asociado a la combinación no rota de generadores viene dado por (4.60). Como $\Theta=\xi-\chi$, entonces

$$
\bar{n}_{\Theta}=\bar{\mu}-\bar{n}_{\chi} .
$$

Pr lo tanto, lo que queremos chequear es la diferencia entre los coeficientes dominantes y subdominantes de $\chi$ como función de la temperatura. El resultado numérico se muestra en la figura 4.10 .

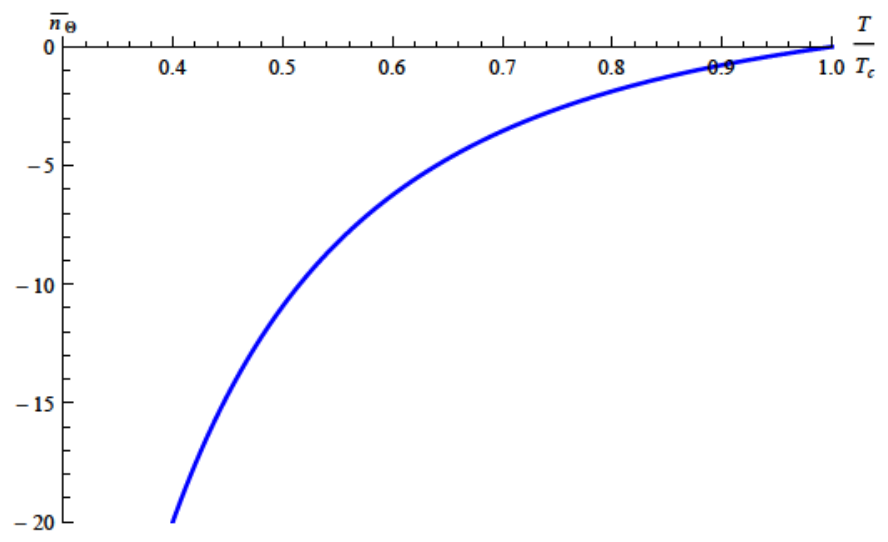

Figura 4.10: Densidad de carga de $\Theta, \bar{n}_{\Theta}$, como función de la temperatura $T / T_{c}$.

Entonces concluimos que a la temperatura $T \leq T_{c}$ esta diferencia se prende y un valor de espectación para $\left\langle Q_{3}\right\rangle$ aparece. Esto puede ser tomado como una clara indicación de la aparición de bosones de Goldstone tipo II en el espectro.

\subsubsection{Fluctuaciones del modelo gaugeado}

En orden de estudiar el espectro de quasipartículas, prendemos las perturbaciones longitudinales sobre el background, como para

$$
\begin{aligned}
\hat{\Psi}^{T} & =(\eta(t, \rho, x), \Psi(\rho)+\sigma(t, \rho, x)), \\
A^{(0)} & =\left(\Phi(\rho)+a_{t}^{(0)}(t, \rho, x)\right) d t+a_{x}^{(0)}(t, \rho, x) d x, \\
A^{(1)} & =a_{t}^{(1)}(t, \rho, x) d t+a_{x}^{(1)}(t, \rho, x) d x, \\
A^{(2)} & =a_{t}^{(2)}(t, \rho, x) d t+a_{x}^{(2)}(t, \rho, x) d x, \\
A^{(3)} & =\left(\Theta(\rho)+a_{t}^{(3)}(t, \rho, x)\right) d t+a_{x}^{(3)}(t, \rho, x) d x .
\end{aligned}
$$




\section{Perturbaciones en la fase no rota}

En la fase normal, el valor de background del condensado se anula. Más aun, tenemos $\Theta(\rho)=0$. Las ecuaciones de movimiento de las perturbaciones son

$$
\begin{aligned}
s^{\prime \prime}+s^{\prime}\left(\frac{f^{\prime}}{f}+\frac{2}{\rho}\right)+\left(\frac{\left(\frac{\Phi}{2}+\omega\right)^{2}}{f^{2}}-\frac{k^{2}}{f \rho^{2}}-\frac{m^{2}}{f}\right) s & =0, \\
a_{t}^{\prime \prime(c)}+\frac{2}{\rho} a_{t}^{\prime(c)}-\frac{\omega k}{f \rho^{2}} a_{x}^{(c)}-\frac{k^{2}}{f \rho^{2}} a_{t}^{(c)} & =0, \\
a_{x}^{\prime \prime(c)}+\frac{f^{\prime}}{f} a_{x}^{\prime(c)}+\frac{\omega^{2}}{f^{2}} a_{x}^{(c)}+\frac{\omega k}{f^{2}} a_{t}^{(c)} & =0, \\
\frac{\omega}{f} a_{t}^{(c)}+\frac{k}{\rho^{2}} a_{x}^{(c)} & =0,
\end{aligned}
$$

donde $s \in\{\eta, \sigma\}$. Como los índices de color no se ven uno al otro el sistema es el mismo que (4.30)-(4.33) con la excepción de que hay cuatro copias del escalar. Debido a nuestra elección de la normalización de los generadores de $U(2)$ el campo de background $\Phi$ aparece con un factor adicional $\frac{1}{2}$ en comparación con (4.30). El espectro de QNM es el mismo que aquel de un superconductor holográfico s-wave [100] conla exepción que los modos escalares están doblemente degenerados y que los modos de los campos de gauge tienen cuatro copias. En particular hay cuatro copias del modo hidrodinámico difusivo $\omega=-i D k^{2}$.

\section{Perturbaciones en la fase rota}

Las ecuaciones de movimiento en la fase rota se desacoplan en dos sectores: uno mezcla de colores $(0)-(3)$ del campo de gauge y fluctuaciones $\sigma$ y el otro mezclando los colores (1) - (2) y las fluctuaciones $\eta$. 
Escribiendo $\sigma=\zeta+i \delta$, las ecuaciones del sector $(0)-(3)$ son

$$
\begin{aligned}
0= & f \zeta^{\prime \prime}+\left(f^{\prime}+\frac{2 f}{\rho}\right) \zeta^{\prime}+\left(\frac{\omega^{2}}{f}+\frac{\chi^{2}}{f}-\frac{k^{2}}{\rho^{2}}-m^{2}\right) \zeta+\frac{2 i \omega \chi}{f} \delta+\left(a_{t}^{(0)}-a_{t}^{(3)}\right) \Psi \frac{\chi}{f}(4 . \\
0= & f \delta^{\prime \prime}+\left(f^{\prime}+\frac{2 f}{\rho}\right) \delta^{\prime}+\left(\frac{\omega^{2}}{f}+\frac{\chi^{2}}{f}-\frac{k^{2}}{\rho^{2}}-m^{2}\right) \delta-\frac{2 i \omega \chi}{f} \zeta+i \Psi \omega \frac{a_{t}^{(3)}-a_{t}^{(0)}}{2 f}+ \\
& +i \Psi k \frac{a_{x}^{(3)}-a_{x}^{(0)}}{2 \rho^{2}} \\
0= & f a_{t}^{\prime \prime(0)}+\frac{2 f}{\rho} a_{t}^{\prime(0)}-\left(\Psi^{2}+\frac{k^{2}}{\rho^{2}}\right) a_{t}^{(0)}-\frac{\omega k}{\rho^{2}} a_{x}^{(0)}+\Psi^{2} a_{t}^{(3)}-4 \zeta \Psi \chi-2 i \omega \Psi \delta \\
0= & f a_{x}^{\prime \prime(0)}+f^{\prime} a_{x}^{\prime(0)}+\left(\frac{\omega^{2}}{f}-\Psi^{2}\right) a_{x}^{(0)}+\frac{\omega k}{f} a_{t}^{(0)}+\Psi^{2} a_{x}^{(3)}+2 i k \delta \Psi \\
0= & f a_{t}^{\prime \prime(3)}+\frac{2 f}{\rho} a_{t}^{\prime(3)}-\left(\Psi^{2}+\frac{k^{2}}{\rho^{2}}\right) a_{t}^{(3)}-\frac{\omega k}{\rho^{2}} a_{x}^{(3)}+\Psi^{2} a_{t}^{(0)}+4 \zeta \Psi \chi+2 i \omega \Psi \delta \\
0= & f a_{x}^{\prime \prime(3)}+f^{\prime} a_{x}^{\prime(3)}+\left(\frac{\omega^{2}}{f}-\Psi^{2}\right) a_{x}^{(3)}+\frac{\omega k}{f} a_{t}^{(3)}+\Psi^{2} a_{x}^{(0)}-2 i k \delta \Psi \\
0 & \frac{i k}{\rho^{2}} a_{x}^{\prime(0)}+\frac{i \omega}{f} a_{t}^{\prime(0)}+2 \Psi^{\prime} \delta-2 \Psi \delta^{\prime} \\
0 & \frac{i k}{\rho^{2}} a_{x}^{\prime(3)}+\frac{i \omega}{f} a_{t}^{\prime(3)}-2 \Psi^{\prime} \delta+2 \Psi \delta^{\prime} .
\end{aligned}
$$

Es trivial mostrar que definiendo los nuevos campos $a_{t}^{( \pm)} \equiv \frac{1}{2}\left(a_{t}^{(0)} \pm a_{t}^{(3)}\right)$ y $a_{x}^{( \pm)} \equiv \frac{1}{2}\left(a_{x}^{(0)} \pm a_{x}^{(3)}\right)$ el sistema se desacopla aun más formando un sistema acoplado para los modos escalares y $a_{\mu}^{(-)}$ y una ecuación independiente del background para el campo de gauge $a_{\mu}^{(+)}$correspondiente al $U(1)$ sobreviviente. El primer subsistema reproduce las ecuacione (4.37)-(4.41) y por lo tanto corresponde al superconductor holográfico s-wave $U(1)$ contenido en el modelo $U(2)$. Por otro lado, el campo $a_{\mu}^{(+)}$corresponde a la simetría de gauge preservada en el proceso de rotura espontánea de simetría $U(2) \rightarrow U(1)$. El espectro de QNMs en este sector es po lo tanto el mismo que presentado en [100] más los QNMs que salen de considerar un gauge field $U(1)$ en $A d S_{4}$. En particular, los modos hidrodinámicos en este sector son el modo de sonido y el modo de sonido asociado al $U(1)$ remanente.

De ahora en adelante nos vamos a concentrar en los campos restantes. Vamos a llamar a este sector, inherentemente no abeliano, sector (1) - (2) y vamos a mostrar que los bosones de Goldstone tipo II que esperamos ver residen acá. Escribiendo $\eta=\alpha+i \beta$, encontramos 
las siguientes ecuaciones de movimiento en el sector $(1)-(2)$ :

$$
\begin{aligned}
0= & f \alpha^{\prime \prime}+\left(f^{\prime}+\frac{2 f}{\rho}\right) \alpha^{\prime}+\left(\frac{\omega^{2}}{f}+\frac{(\Phi+\Theta)^{2}}{4 f}-\frac{k^{2}}{\rho^{2}}-m^{2}\right) \alpha+\frac{i \omega(\Phi+\Theta)}{f} \beta- \\
& -i \Psi\left(\frac{k}{2 \rho^{2}} a_{x}^{(2)}+\frac{\omega}{2 f} a_{t}^{(2)}\right)+\frac{\Psi \Phi}{2 f} a_{t}^{(1)} \\
0= & f \beta^{\prime \prime}+\left(f^{\prime}+\frac{2 f}{\rho}\right) \beta^{\prime}+\left(\frac{\omega^{2}}{f}+\frac{(\Phi+\Theta)^{2}}{4 f}-\frac{k^{2}}{\rho^{2}}-m^{2}\right) \beta-\frac{i \omega(\Phi+\Theta)}{f} \alpha- \\
& -i \Psi\left(\frac{k}{2 \rho^{2}} a_{x}^{(1)}+\frac{\omega}{2 f} a_{t}^{(1)}\right)-\frac{\Phi \Psi}{2 f} a_{t}^{(2)}, \\
0= & f a_{t}^{\prime \prime(1)}+\frac{2 f}{\rho} a_{t}^{\prime(1)}-\left(\Psi^{2}+\frac{k^{2}}{\rho^{2}}\right) a_{t}^{(1)}-\frac{\omega k}{\rho^{2}} a_{x}^{(1)}+i \Theta \frac{k}{\rho^{2}} a_{x}^{(2)}-2 \Phi \Psi \alpha-2 i \omega \Psi \beta,(1) \\
0= & f a_{x}^{\prime \prime(1)}+f^{\prime} a_{x}^{\prime(1)}+\left(\frac{\omega^{2}}{f}-\Psi^{2}+\frac{\Theta^{2}}{f}\right) a_{x}^{(1)}-2 i \frac{\Theta \omega}{f} a_{x}^{(2)}-i \Theta \frac{k}{f} a_{t}^{(2)}+\frac{\omega k}{f} a_{t}^{(1)} \beta+ \\
& +2 i k \Psi \beta, \\
0= & f a_{t}^{\prime \prime(2)}+\frac{2 f}{\rho} a_{t}^{\prime(2)}-\left(\Psi^{2}+\frac{k^{2}}{\rho^{2}}\right) a_{t}^{(2)}-\frac{\omega k}{\rho^{2}} a_{x}^{(2)}-i \Theta \frac{k}{\rho^{2}} a_{x}^{(1)}+2 \Phi \Psi \beta-2 i \omega \Psi \alpha,(4.8) \\
0= & f a_{x}^{\prime \prime(2)}+f^{\prime} a_{x}^{\prime(2)}+\left(\frac{\omega^{2}}{f}-\Psi^{2}+\frac{\Theta^{2}}{f}\right) a_{x}^{(2)}+2 i \frac{\Theta \omega}{f} a_{x}^{(1)}+i \Theta \frac{k}{f} a_{t}^{(1)}+\frac{\omega k}{f} a_{t}^{(2)}+ \\
& +2 i k \Psi \alpha, \\
0= & \frac{i k}{\rho^{2}} a_{x}^{\prime(1)}+\frac{i \omega}{f} a_{t}^{\prime(1)}+\frac{1}{f}\left(a_{t}^{\prime(2)} \Theta-a_{t}^{(2)} \Theta^{\prime}\right)+2 \Psi^{\prime} \beta-2 \beta^{\prime} \Psi \\
0 & \frac{i k}{\rho^{2}} a_{x}^{\prime(2)}+\frac{i \omega}{f} a_{t}^{\prime(2)}-\frac{1}{f}\left(a_{t}^{\prime(1)} \Theta-a_{t}^{(1)} \Theta^{\prime}\right)+2 \Psi^{\prime} \alpha-2 \alpha^{\prime} \Psi . \\
0 & \\
0 &
\end{aligned}
$$

Un comentarito: El sistema de ecuaciones podrían ser escritas de manera más compacta usando las variables complejas $\eta$ y $a_{t, x}^{(1)} \pm i a_{t, x}^{(2)}$. Uno debe tener en mente en ese caso que las ecuaciones de campo que uno necesita resolver para obtener el espectro de QNMs no son las ecuaciones complejo conjugadastya que uno tiene que pedir condiciones de borde entrantes en el agujero negro y los campos y en los campos complejo conjugados de manera simultánea. Este aspecto es más claro si uno trabaja con los campos (formalmente) reales como variables a costas de que las ecuaciones se vean un poco más largas.

Considerando hasta orden lineal en las ecuaciones, hay tres sectores desacoplados en el sistema. Dos de los cuales pertenecen al 'sector (0) - (3)' y son una copia del superconductor holográfico $U(1)$ ya estudiado extensivamente, y de una simetría de gauge $U(1)$ preservada. Las principales características de este espectro ya fueron presentadas en 4.3 ya que es también un subsector del modelo no gaugeado. Por otro lado, el llamado 'sector (1) - (2)' no fue estudiado aun. La física de este sector es bastante diferente de la física presentada hasta ahora y nos conentraremos en la misma en lo que queda de este capítulo.

Antes de estudiar los modos quasinormales vamos a concentrarnos en un problema más sencillo, el de las conductividades. 


\subsubsection{Conductividades}

Para estudiar conductividades usando al fórmula de Kubo, es suficiente resolver las ecuaciones linealizadas en el límite $k=0$. Los correladores retardados en los que estamos interesados tienen la forma $\mathcal{G}_{\mathcal{R}} \sim\left\langle J_{(c)}^{x}, J_{\left(c^{\prime}\right)}^{x}\right\rangle_{\mathcal{R}}$, con $c, c^{\prime}$ índices de color.

Vamos a usar la prescripción de [119] para computar funciones de Green en presencia de operadores mezclados. Si uno tiene un conjunto de campos $\Phi_{I}$, la función de correlación de dos puntos será

$$
\mathcal{G}_{I J}=\lim _{\Lambda \rightarrow \infty}\left(\mathcal{A}_{I M} \mathcal{F}_{k}^{M}{ }_{J}(\Lambda)^{\prime}+\mathcal{B}_{I J}\right),
$$

donde la matriz $\mathcal{F}_{k}(r)$ no es nada más que el porpagador bulk-to-boundary para los campos, normalizado ed manera que sea la identidad en el borde. Las matrices $\mathcal{A}$ y $\mathcal{B}$ pueden ser leidas de las acción on-shell renormalizada. Las correspondientes conductividades DC son según la fórmula de Kubo

$$
\sigma_{I J}=\lim _{\omega \rightarrow 0}\left(\frac{i}{\omega} \mathcal{G}_{I J}(\omega, 0)\right) .
$$

A momento cero, las componentes longitudinales de los campos de gauge se desacoplan de las perturbaciones escalares, así como de las componentes temporales de los campos de gauge. Más aun, los contraints (eqs. (4.81-4.82) y (4.89-4.90)) resultan satisfacerse de manera trivial. Como sabemos que el sistema se separa en los sectores $(0)-(3)$ ythe $(1)-(2)$ podemos rearreglar a los campos $a_{x}^{(c)}$ en dos vectores

$$
\Phi_{k(0-3)}^{T}(\rho)=\left(a_{x}^{(0)}(\rho), a_{x}^{(3)}(\rho)\right) \quad \text { and } \quad \Phi_{k(1-2)}^{T}(\rho)=\left(a_{x}^{(1)}(\rho), a_{x}^{(2)}(\rho)\right) .
$$

Uno puede verificar que en nuestro caso las matrices $\mathcal{A}, \mathcal{B}$ toman una forma sencilla

$$
\mathcal{A}=-\frac{f(r)}{2} \mathbb{I}, \quad \mathcal{B}=0
$$

en ambios sectores. A priori tendríamos una matriz de $4 \times 4$ de conductividades. Sabemos sin embargo que las fluctuaciones en los sectores (0) - (3) y (1) - (2) se desacoplan unas de las otras. Entonces podemos restringirnos al estudio de dos matrices de $2 \times 2$ de conductividades.

\subsubsection{Conductividades en el sector $(0)-(3)$}

Las ecuaciones de movimiento a $k=0$ para $a_{x}^{(0)}$ y $a_{x}^{(3)}$ pueden ser simplificadas usando los ya definidos campos $a_{x}^{(-)}$y $a_{x}^{(+)}$. Esto resulta en

$$
\begin{aligned}
0 & =f a_{x}^{\prime \prime(+)}+f^{\prime} a_{x}^{(+)}+\frac{\omega^{2}}{f} a_{x}^{(+)} \\
0 & =f a_{x}^{\prime \prime(-)}+f^{\prime} a_{x}^{\prime(-)}+\left(\frac{\omega^{2}}{f}-2 \Psi^{2}\right) a_{x}^{(-)} .
\end{aligned}
$$

Vemos que el sistema de ecuaciones resultante ahora está completamente desacoplado. Sólo tenemos dos conductividades diagonales $\sigma_{++}$y $\sigma_{--}$, correspondientes al sector difusivo $U(1)$ y a un modo asociado con la rotura espontánea de la simetría $U(1)$ que se acopla al condensado. El primero es igual que en la fase no rota y de no mayor interés para nosotros . El último es el del bien estudiado superconductor s-wave $U(1)$. Su conductividad ya fue 
calculada en [28]. Para corroborar el análisis numérico la hemos recomputado y la figura 4.11 muestra su comportamiento. El mismo coincide completamente con [28]. LA parte real muestra la función delta característica de la superconductividad a $\omega=0{ }^{10}$. Numéricamente esto puede ser visto a través del polo simple $1 / \omega$ en la parte imaginaria de la conductividad. La relación de Kramers-Kronig (ver (A.13) en el apéndice A.1) implica entonces conductividad DC infinita. La parte real de la conductividad AC también exibe un gap dependiente de la temperatura.

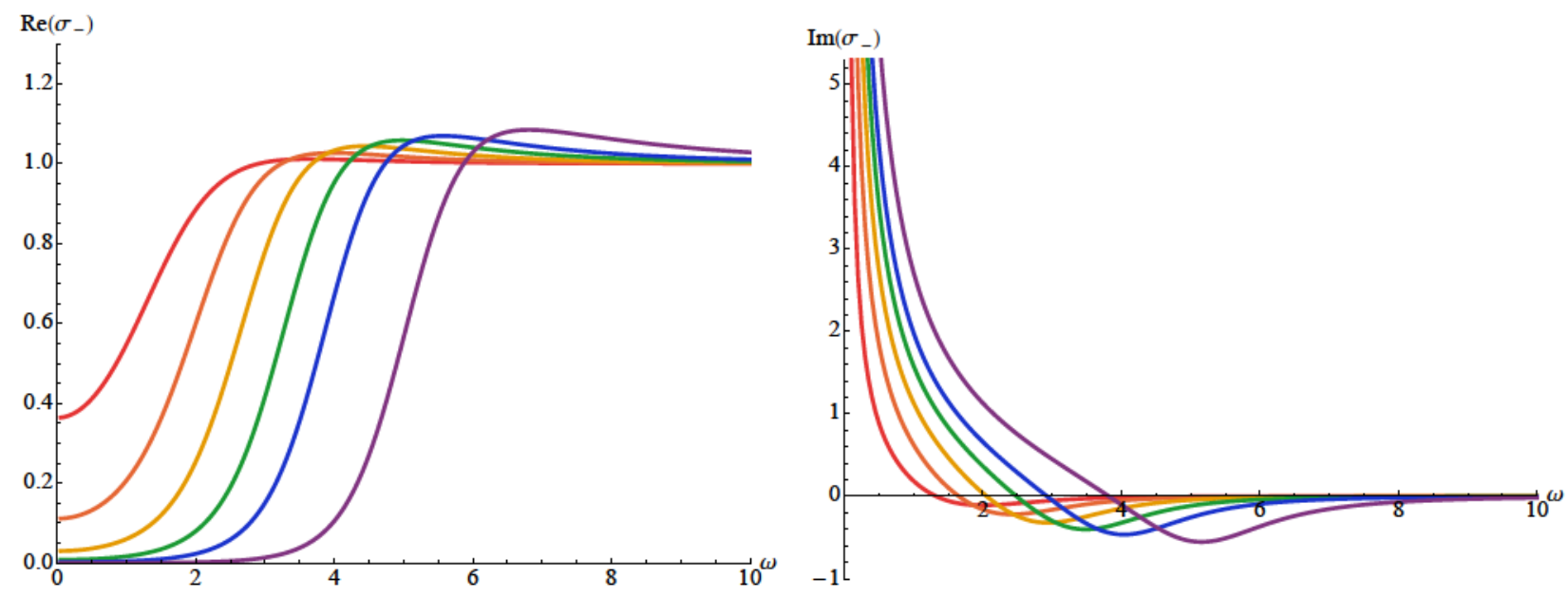

Figura 4.11: Partes real (izquierda) e imaginaria (derecha) de la conductividad como función de la fracuencia. Los gráficos corresponden a temperaturas $T / T_{c} \approx 0,91-0,41$, de rojo a violeta. Como es de esperarse, los gráficos reproducen a aquellos de [28].

\subsubsection{Conductivities in the (1) - (2) sector}

Las ecuaciones relevantes para el sector (1) - (2) son

$$
\begin{aligned}
& 0=f a_{x}^{\prime \prime(1)}+f^{\prime} a_{x}^{(1)}+\left(\frac{\omega^{2}}{f}-\Psi^{2}+\frac{\Theta^{2}}{f}\right) a_{x}^{(1)}-2 i \frac{\Theta \omega}{f} a_{x}^{(2)}, \\
& 0=f a_{x}^{\prime \prime(2)}+f^{\prime} a_{x}^{\prime(2)}+\left(\frac{\omega^{2}}{f}-\Psi^{2}+\frac{\Theta^{2}}{f}\right) a_{x}^{(2)}+2 i \frac{\Theta \omega}{f} a_{x}^{(1)} .
\end{aligned}
$$

Estas ecuaciones obedecen la simetría

$$
\left(a_{x}^{(1)} \rightarrow a_{x}^{(2)}, a_{x}^{(2)} \rightarrow-a_{x}^{(1)}\right) .
$$

Uno puede ver que el hecho que $\Theta(1)=0$ implica que $a_{x}^{(1)}(1)$ es independiente de $a_{x}^{(2)}(1)$, entonces, después de imponer condiciones entrantes en el horizonte, el espacio de parámetros de condiciones de borde es bidimensional, como era de esperarse.

En la fase no orta el sistema se desacopla completamente

$$
0=f a_{x}^{\prime \prime(c)}+f^{\prime} a_{x}^{(c)}+\frac{\omega^{2}}{f} a_{x}^{(c)} .
$$

\footnotetext{
${ }^{10}$ En general, este comportamiento también es característico de medios cargados que gozan de invarianza traslacional, en los cuales cargas aceleradas no pueden relajarse. Sin embargo, al trabajar en el límite de prueba rompemos la invarianza traslacional de manera efectiva y por lo tanto la conductividad DC infinita es una señal aunténtica de superconductividad.
} 
Conductividades diagonales $\sigma^{11} \& \sigma^{22}$

Las componentes diagonales de la conductividad, $\sigma^{11}$ y $\sigma^{22}$ tienen el mismo comoportamineto, como puede ser anticipado de las ecuaciones (4.97),(4.98). Por lo tanto, vamos a referirnos solamente a $\sigma^{11}$, pero todas las conclusiones se aplican también a $\sigma^{22}$.

La figura 4.12 muestra la conductividad para varios valores de la temperatura. Encontramos que estas conductividades también muestras singularidades tipo delta a $\omega=0$.
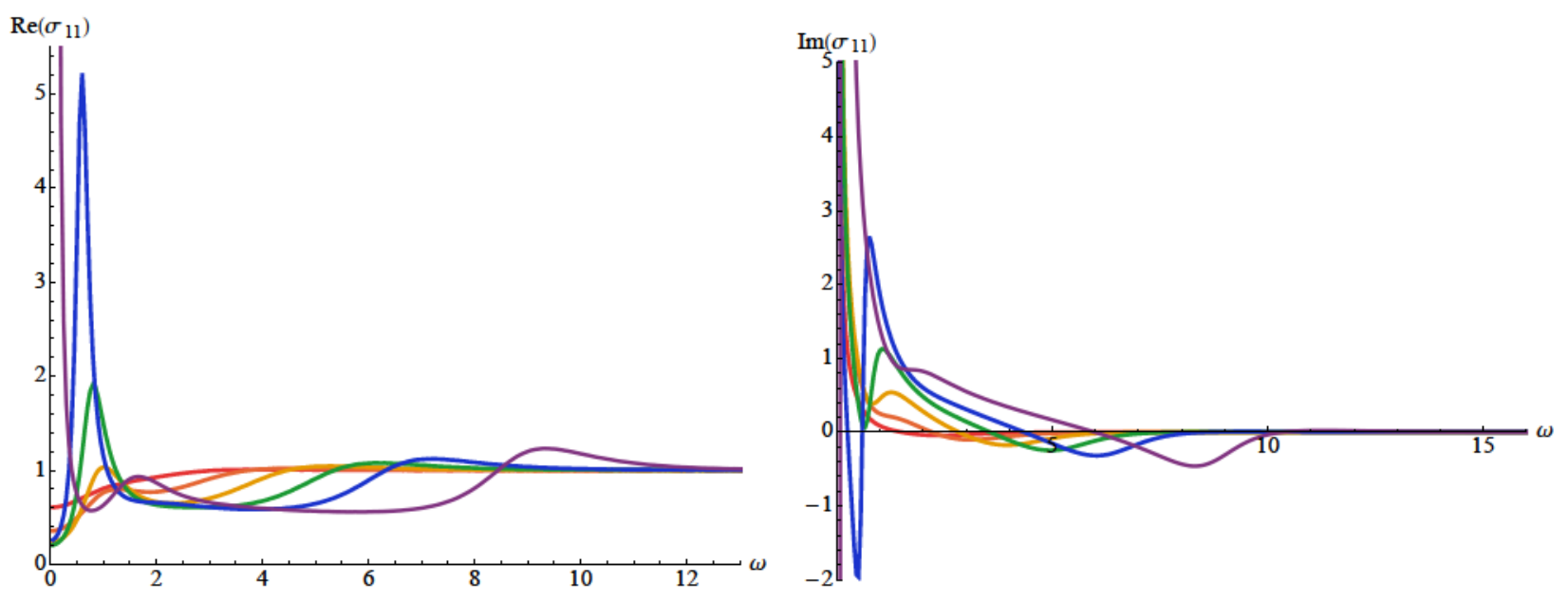

Figura 4.12: Partes real (izquierda) e imaginaria (derecha) de $\sigma^{11}$ versus $\omega$ para cinco temperaturas diferentes elegidas en un rango $T / T_{c} \approx 0,91-0,41$, de rojo a violeta. $\operatorname{Im}\left(\sigma^{11}\right)$ claramente explota a medida que $\omega \rightarrow 0$.

El poder de la función delta también puede ser calculado. Viene dado por el residuo de la parte imaginaria de al conductividad a $\omega=0$,

$$
\lim _{\omega \rightarrow 0} \omega \operatorname{Im}\left(\sigma^{11}\right) \sim n_{s} .
$$

Este residu es dibujado en la figura 4.13 como función de $T / T_{c}$. Como es de esperarse, empieza a crecer desde un valor nulo. A $T / T_{c} \approx 0,65, n_{s}$ alcanza un máximo y empieza a decrecer rápidamente, cambiando de signo a $T / T_{c}=0,49$. Para estudiar $n_{s}$ hasta temperaturas muy bajas deberíamos ir más allá del límite de prueba. Sin embargo, como vamos a comentar más abajo, este comportamiento de $n_{s}$ puede ser entendido a la luz del espectro de QNMs.

Miremos en detalle el comportamiento de la parte real de la conductividad (gráfico de la izquierda en la figura 4.12). Para temperaturas lo suficientemente altas la conductividad óptica, $\operatorname{Re}\left(\sigma^{11}\right)=1$, lo que es de esperarse ya que para ese régimen la dinámica vienen gobernada por (4.100). Apenas bajamos la temperatura, el comienzo (bajas frecuencias) decrece y soo se acerca a un valor constante asintóticamente, cuando $\omega$ se hace los suficientemente grande y entonces el término $\frac{\omega^{2}}{f}$ domina, tranformando las ecuaciones $(4.97),(4.98)$ en aproximadamente (4.100). De acuerdo con la regla de suma de Ferrell-Glover el área que se pierde a medida que bajamos la temperatura es proporcional a $n_{s}$.

Notablemente, a bajas temperaturaas la parte real de $\sigma^{11}$ empieza a desarrollar una protuberancia a $\omega(0<\omega \lesssim 2)$. Esta protuberancia le deja menso área que cubrir a la función delta, lo que explica por qué $n_{s}$ empieza a decrecer a aproximadamente esta temperatura. 


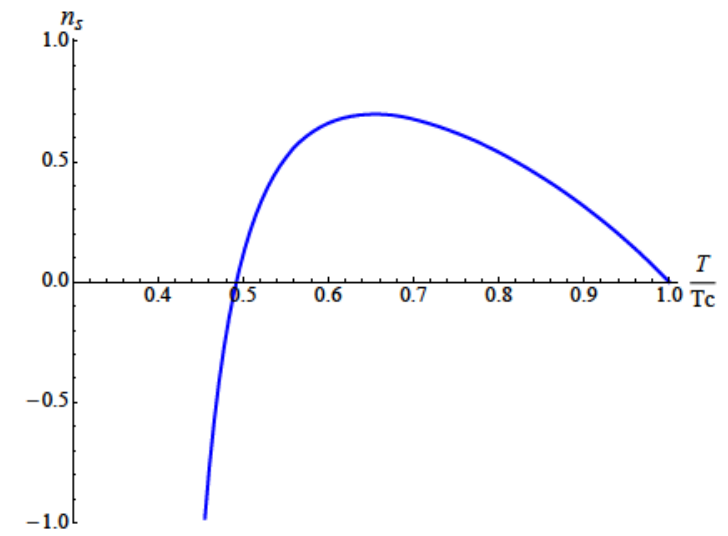

Figura 4.13: Residuo a $\omega=0$ como función de $T / T_{c}$.

Más aun, la aparición de estas protuberancias puede ser asociada al hecho que para qNM de gauge con $|\operatorname{Im}(\omega)|$ pequeña, vale que $\operatorname{Re} \omega(T)>>\operatorname{Im} \omega(T)$. Entonces, las conductividades se ven afectadas por el hecho de que este modo empiece a desarrollar una especie de resonancia a esa frecuencia en particular. Hemos estudiado el espectro de los QNM más bajos para el sector de gauge y encontramos que este modo corresponde en la fase normal a la excitación más baja de $a_{\mu}^{(1,2)}, \omega=-1,5 i$. Pero es a temperaturas más bajas que uno encuentra un hecho remarcable: a $T / T_{c} \approx 0,395$ los modos se vuelven inestables, y de hecho, como veremos, varias cantidades físicas modifican su comportamiento a esa temperatura.

Por lo tanto, esperamos una nueva transición de fase alrededor de $T / T_{c} \approx 0,395$, debido enteramente al sector $(1)-(2)$. Como esta transición de fase parece ser disparadas por un modo en el sector vectorial lo más probable es que lleve a la formación de un condensado tipo p-wave. Esta nueva fase será detallada en el capítulo 5.

\section{Conductividades fuera de la diagonal $\sigma^{12} \& \sigma^{21}$}

Los elementos fuera de la diagonal de la matriz de conductividades están también relacionados a través de la simetría (4.99) y por lo tanto obedecen $\sigma^{12}=-\sigma^{21}$. Entonces, es suficiente hacer comentarios para $\sigma^{12}$, aunque las conclusiones van a ser válidas para ambas componentes.

La forma de $\sigma^{12}$ es graficada en la figura 4.14 para varias temperaturas diferentes como función de la frecuencia. A $T / T_{c}=1$ el sistema está prácticamente desacoplado, entonces para todas las temperaturas las conductividades diagonales van a cero a medida que $\omega$ aumenta.

Obsérvese que $\sigma^{12}(\omega)$ se comporta como una conductividad normal. Su parte real se anula para $\omega \rightarrow 0$, mientras que la imaginaria tiende a un valor constante.

\section{Conductivities $\sigma_{+-}$and $\sigma_{-+}$}

Vale la pena hacer notar que las conductividades (4.97)-(4.98) se desacoplan si definimos un nuevo campo vectorial

$$
\tilde{\varphi}=\left(\begin{array}{c}
A_{+} \\
A_{-}
\end{array}\right)=\left(\begin{array}{cc}
1 & i \\
1 & -i
\end{array}\right)\left(\begin{array}{l}
a_{x}^{(1)} \\
a_{x}^{(2)}
\end{array}\right)=S \varphi .
$$




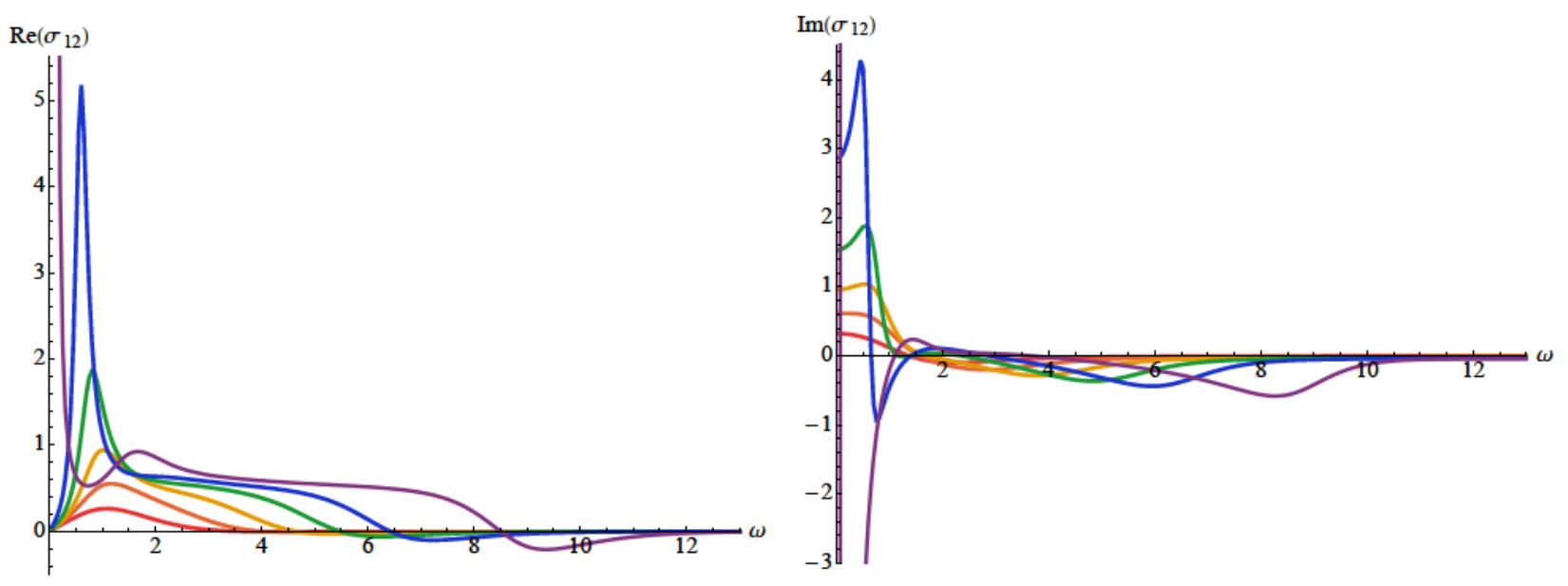

Figura 4.14: Partes real (izquierda) e imaginarias (derecha) de $\sigma^{12}$ como función de $\omega$ para $T / T_{c} \approx 0,91-0,41$, de rojo a violeta.

En esta base, las ecuaciones de movimiento son

$$
0=f A_{ \pm}^{\prime \prime}+f^{\prime} A_{ \pm}^{\prime}+\left(\frac{(\omega \mp \Theta)^{2}}{f}-\Psi^{2}\right) A_{ \pm}
$$

Es fácil corroborar que la relación entre las matrcies de conductividades en ambas bases viene dada por

$$
\tilde{\sigma}=\left(S^{T}\right)^{-1} \sigma S^{-1}
$$

y que sólo las partes no diagonales de $\tilde{\sigma}$ son no nulas.

Las conductividades $\sigma_{-+}$y $\sigma_{+-}$están representadas en las figuras 4.15 y 4.16 , respectivamente. El plot de la conductividad $\sigma_{-+}$es particularmente sugestivo. Aparte de la delta superconductora de la conductividad DC nos recuerda al comportamiento observado en Grafeno [114]. Semejante similutud entre las conductividades de superconductores holográficos y Grafeno fue ya señalada en [1]. Enfatizamos sin embargo que en la figura 4.15 tenemos una semejanza aun más grande con el Grafeno [114], la cual puede observarse en la figura 4.17. En particular, a bajas frecuencias vemos que se desarrolla un pico tipo Drude. Este tipo de comportamiento en metal por lo general es asociado a impurezas o a la existencia de una red, mientras que en nuestro caso, la relajación de momento vendría dada por un valor de espectación no nulo del operador densidad de carga $\left\langle Q_{3}\right\rangle=\bar{n}_{\Theta}$. Para frecuencias grandes, el hecho de que la conductividad vaya a una constante es universal en sistemas en $2+1$ dimensiones, y está asociado a que la conductividad no tiene unidades en esta elección particular de dimensión.

La similitud se mantiene para temperatura no demasiado pequeñas. Cuando bajamos la temperatura, se abre un gap para el sector $(0)-(3)$. La parte real de $\sigma_{+-}$muestra el mismo pico que $\sigma_{11}$ cuando bajamos la temperatura. Para temperaturas por debajo de $T \approx 0,49 T_{c}$, el polo en la parte imaginaria de ambas conductividades cambia de signo. Por supuesto, corresponde a la temperatura para la cual el residuo cambia de signo. El comportamiento a bajas frecuencias de la conductividad crece rápidamente a medida que bajamos la temperatura, y se hace divergente a $T / T_{c} \approx 0,395$. La presencia de ese polo en la conductividad está relacionado con la aparición de una inestabilidad en el espectro 
de exitaciones de la teoría de campos y por lo tanto con la transición de a una nueva fase superconductora, como fue ya discutido.
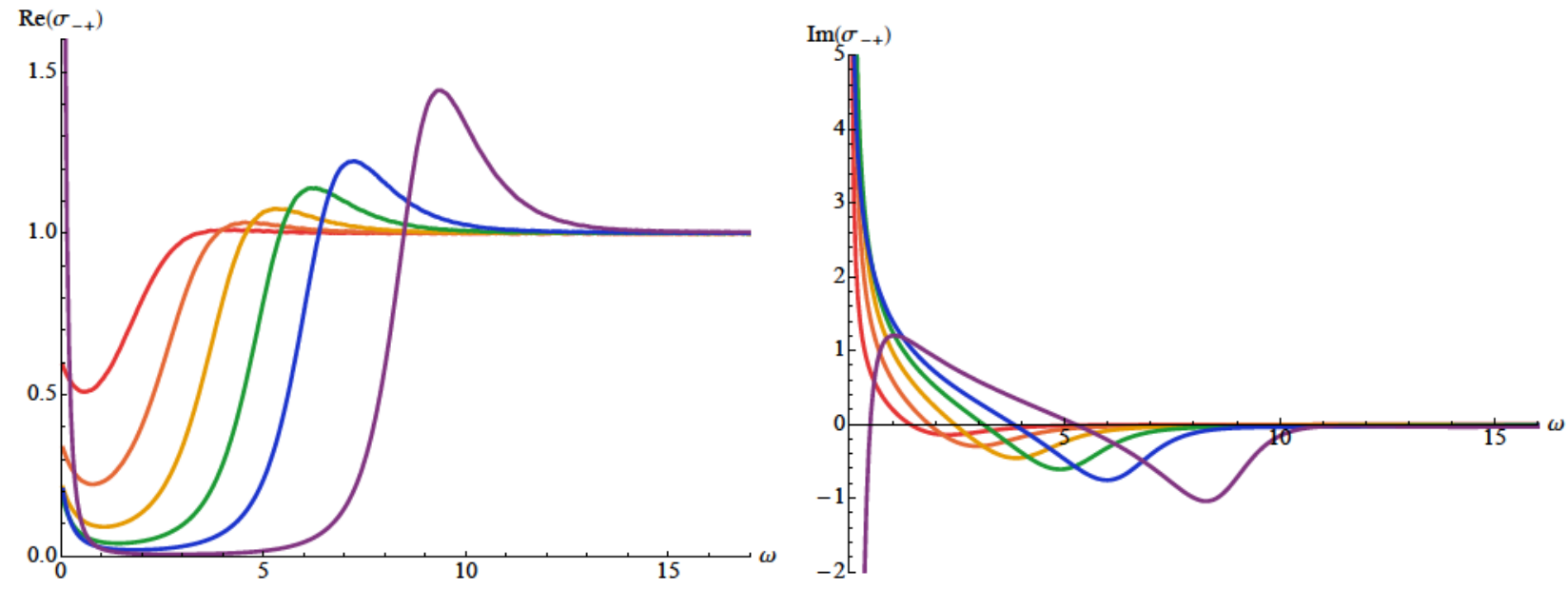

Figura 4.15: Partes real (izquierda) e imaginaria (derecha) de la conductividad $\sigma_{-+}$para temepraturas en el rango $T / T_{c} \approx 0,91-0,41$, de rojo a violeta.
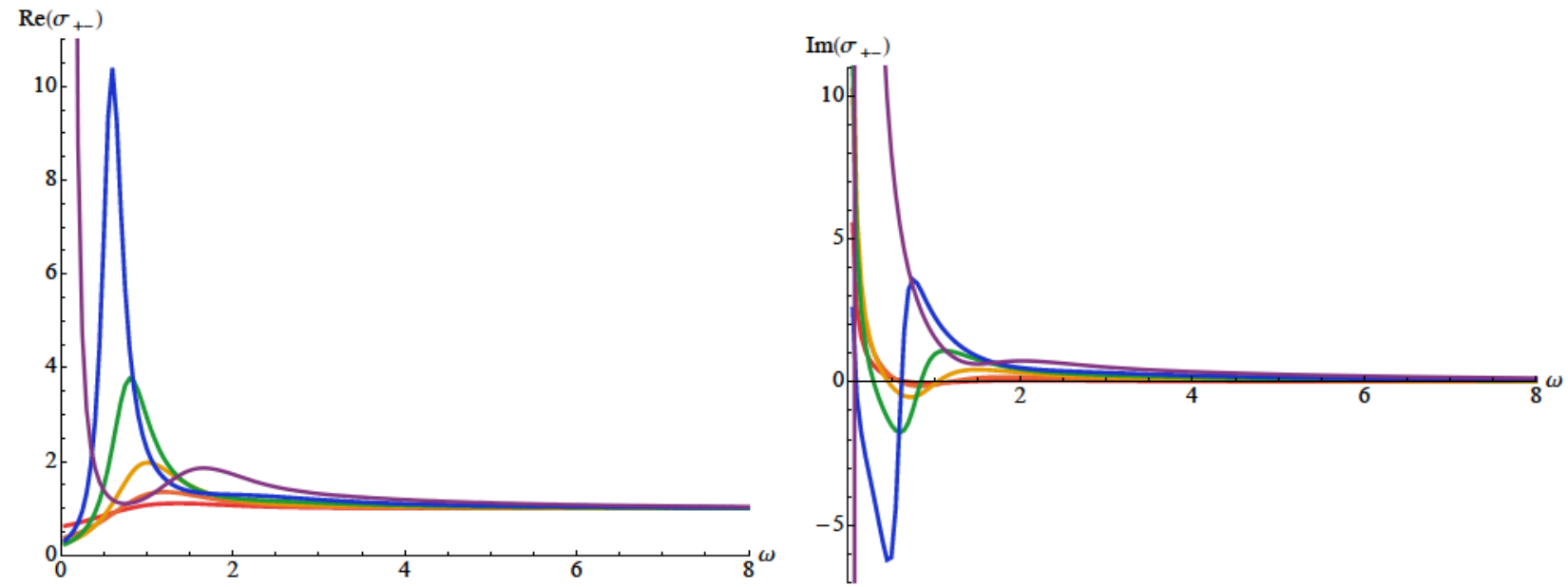

Figura 4.16: Partes real (izquierda) e imaginaria (derecha) de la conductividad $\sigma_{+-}$para temepraturas en el rango $T / T_{c} \approx 0,91-0,41$, de rojo a violeta.

\subsubsection{Modos quasinormales}

Vamos finalmente a estudiar el espectro de QNM del sector (1)-(2). Este sector contiene las fluctuaciones $\eta, a_{\mu}^{i}$ con $i=1,2$, y por ello en la fase rota el espectro va a contener dos modos difusivos asociados con los dos campos de guage. Las fluctuaciones del campo escalar en la fase normal fueron ya discutidas en 4.3. Analizando el espectro de modos quasinormales en la fase rota consiste en resolver el sistema de ecuaciones (4.83)-(4.90). Detalles de dicha cuenta pueden encontrarse en el apéndice A.2. 

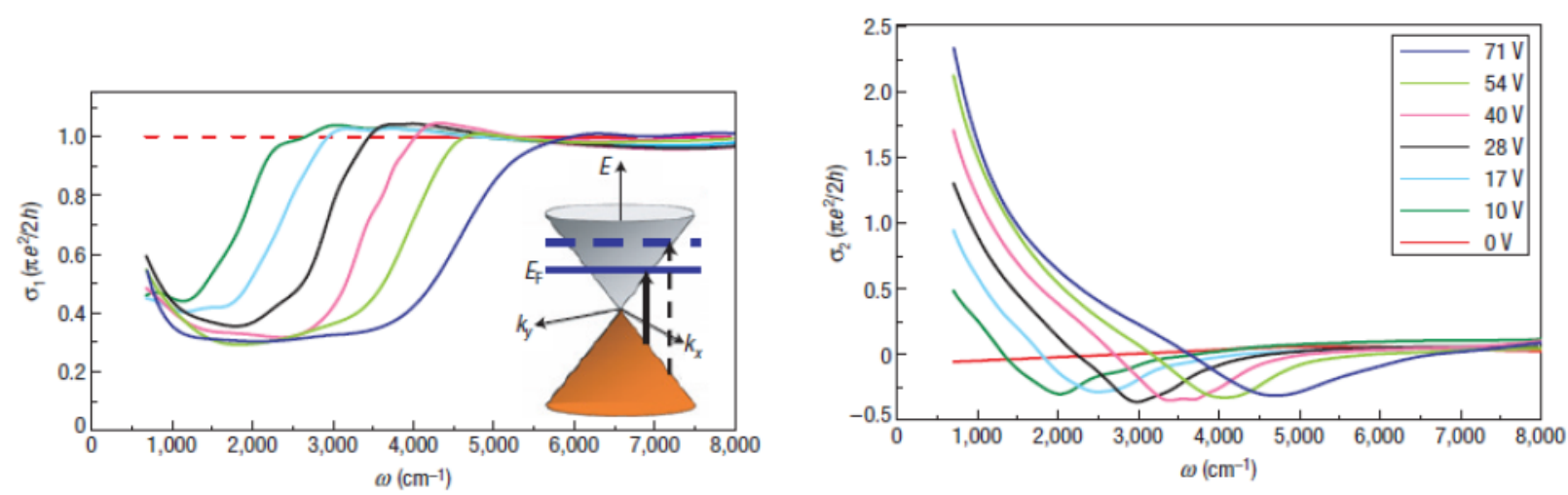

Figura 4.17: Partes real (izquierda) e imaginaria (derecha) de la conductividad para el Grafeno. Variar el potencial $V$ es equivalente de alguna manera a cambiar $1 / T$ en nuestro modelo holográfico.

\section{Modo de Goldstone tipo II}

Como es de esperarse en el sector (1) - (2) encontramos un modo de Goldstone del tipo II. Así como en el modelo sin gaugear, su comportamiento para momentos pequeños se puede fitear con

$$
\omega= \pm \mathcal{B} k^{2}-i \mathcal{C} k^{2}
$$

La figura 4.18 muestra la relación de dispersión para varias teperaturas y el fit en el régimen hidrodinámico.
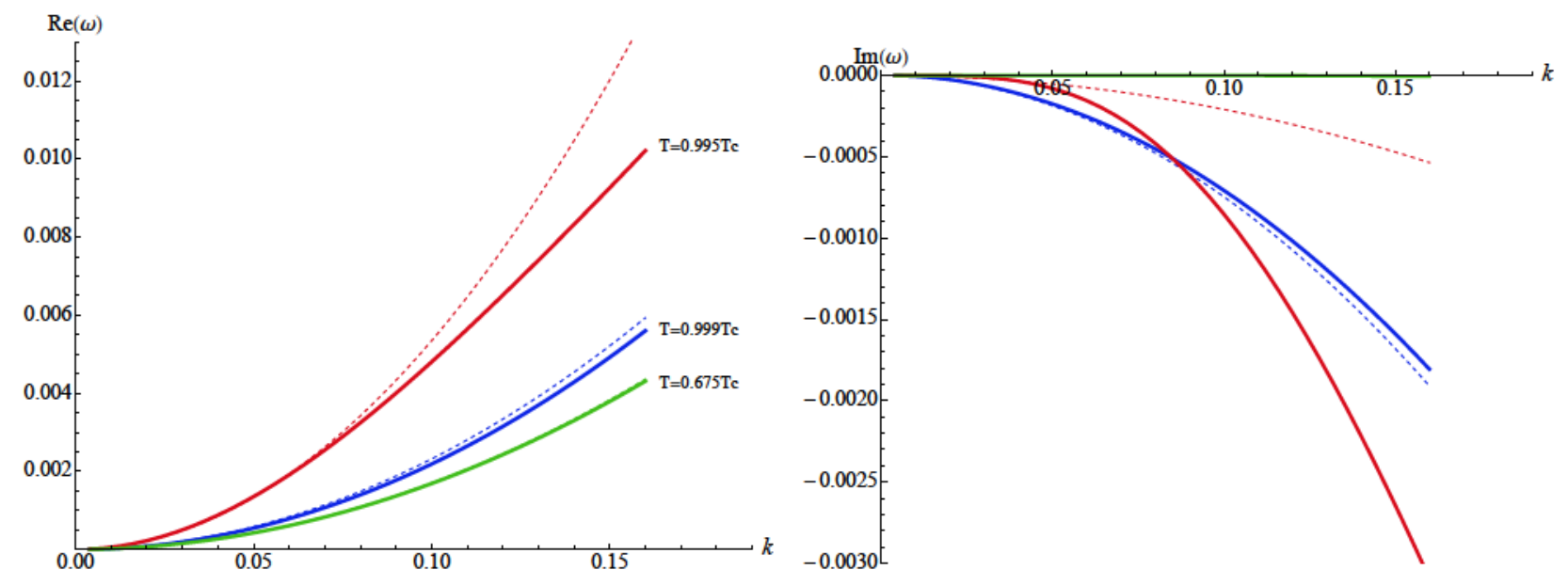

Figura 4.18: Gráficos de $\operatorname{Re}(\omega)$ (izquierda) y $\operatorname{Im}(\omega)$ (derecha) como función del momento. Líneas gruesas representan data numérica mientras que las líneas delgadas corresponden a ajustes. A $T=0,995 T_{c}$ el parámetro real cuadrático $\mathcal{B}(T)$ muestra un máximo, véase la figura 4.19. La relación (4.105) es satisfecha con gran precisión.

La dependencia en temperatura de $\mathcal{B}$ y $\mathcal{C}$ se muestra graficada en la figura 4.19. El valor a $T=T_{c}$ el el mismo que para el modelo no gaugeado (4.46) y de hecho puede ser cross checked mediante el cálculo de la relación de dispersión del modo escalar en la 
fase no rota a $T=T_{c}$ ya que los QNMs deben ser continuos a través de la transición de fase. Encontramos una dependencia bastante sorprendente de $\mathcal{B}$ con la temperatura. Comienza a un valor finito a la temperatura crítica y luego aumenta de manera bursca para finalmnte caer lentamente. Llega a un mínimo aproximandamente para $T \approx 0,49 T_{c}$, temeperatura a la cual encontramos el cambio de signo de los correladores corriente-corriente. También encontramos otro pico alrededor $T \approx 0,4 T_{c}$. Esperamos que se encuentre una vez más relacionado con la inestabilidad encontrada en el secotr de gauge alrededor de esa temperatura. Sería interesante calcular $\mathcal{B}(T)$ usando algún otro método e.g. ya que la velocidad del sonido puede ser calculada usando métodos puramente termodinámicos. Para hacer esto uno debería ser capaz de conocer la hidrodinámica de los modos de Goldstone de tipo II. Siun embargo desconocemos dicha formulación hidrodinámica y dejamos dicha pregunta para investigación futura.
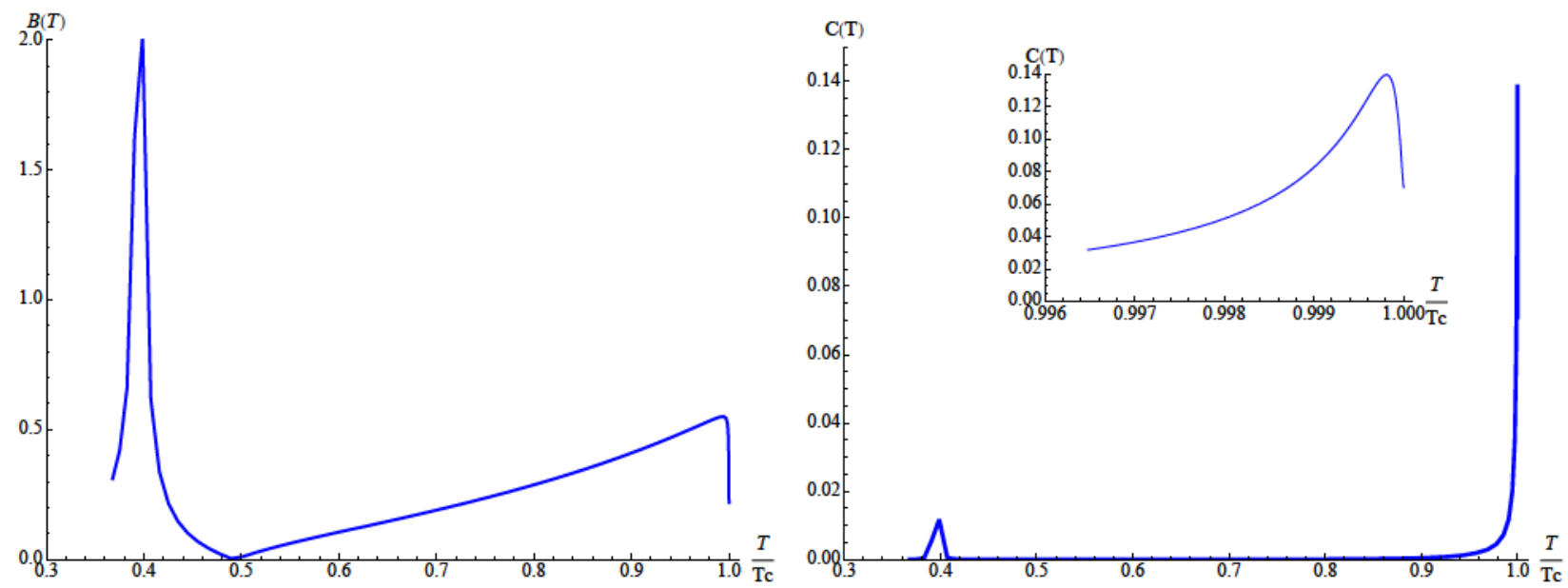

Figura 4.19: $\mathcal{B}$ (izquierda) y $\mathcal{C}$ (derecha) como función de $T / T_{c}$. Al hacer zoom vemos el pico para $\mathcal{C}$ cerca de la transición. Más aun, a $T \simeq 0,4 T_{c}$ un pico agudo aparece en ambos coeficientes. Atribuímos esta manifestación al hecho de que aparece una inestabilidad para el modo vectorial a dicha temperatura.

La atenuación $\mathcal{C}(T)$ decrece rápidamente con la temeperatura. Para temperaturas $T / T_{c}<$ 0,9 es despreciable y el ancho de los bosones de Goldstone tipo II decae como $k^{4}$ en el límite hidrdinámico. Este rápido decrecimiento con la temperatura manifiesta que el modo se propaga de manera casi ideal a temperaturas bajas. No se han encontrado otros modos no masivos en este sector.

\section{Modos quasinormales más altos}

Modos quasinormales más altos corresponden a modos masivos enel espectro de QNM representando por lo tanto contribuciones subleading a las funciones de Green de bajas energías. Nos vamos a concentrar en sólo dos de ellos: La continuación de los dos modos difusivos de la fase normal y el modo masivo especial que aparece como modo compañero del bosón de Goldstone tipo II en el modelo de teoría de campos.

Analizar el primero es interesante para comprender si un cambio cualitativo en el patrón de respuesta, como aquel caracterizado por $T_{*}$ en el sector superconductor $U(1)$, existe en el sector (1) - (2). Como en este sector existen sin embargo dos modos difusivos en la fase no 
rota es posible que los modos difusivos no solo desarrollen un gap, sino que se acplen entre ellos y se muevan fuera del eje imaginario en la fase rota. Veremos que este es efectivamente el caso.

El modo especial gapeado corresponde a un modo que es asociado al complejo conjugado de la perturbación escalar en la fase normal. A $k=0$ y $\mu=0$ el modo escalar y su complejo conjugado están degenerados. A medida que bajamos la temperatura se separan en dos modos diferentes. Cuando llegamos a $T=T_{c}$, el modo escalar más bajo se convierte en el bosón de GOldstone tipo II mientras que el modo del complejo conjugado se convierte en su compañero masivo. El gap de este modo se espera que esté dado por su resultado a tree-level (4.13) [116].

El destino de los modos difusivos: Como ya fue mencionado, en el sector (1) - (2) tenemso dos modos difusivos degenerados en la fase rota. Cuando atravesamos una transición de fase estos modos pueden por lo tanto aparearse y moverse fuera del eje imaginario de manera tal que sus frecuencias quasinormales desarrollen partes reales y queden paradas de manera simétrica alrededor del eje imaginario. Esperamos entonces que en el límite de bajas energías la relación de dispersión tome la forma

$$
\omega=\Gamma(T)+\mathcal{M}(T) k^{2},
$$

donde los dos coeficientes son funciones compleja y el segundo modo está localizado a $\omega^{\prime}=$ $-\omega^{*}$. Además, esperamos que los QNMs sean continuos a través de la transición de fases, lo que en particular significa que para $T=T_{c}$, nuestro modo pseudodifusivo se pegue con el valor para el modo difusivo en la fase rota, i.e. $\Gamma\left(T_{c}\right)=0$ y $\mathcal{M}\left(T_{c}\right)=-i$.

Los modos a momento cero están graficados en la figura 4.20. Vemos que de hecho el gap se anula a $T \rightarrow T_{c}$, mientras que los modso se separan y desarrollan un parte real a medida que disminuimos la temperatura. Esta última característica es exclusiva de un sistema no abeliano y por lo tanto no ocurre en el superconductor holgráfico $U(1)$ de toda la vida, donde el gap es puramente imaginario (ver [100] y los comentarios hechos más arriba). Cerca de la transición de fase, ellos presentan un comportamiento lineal en la temperatura,

$$
\Gamma(T)=(4,1-0,8 i)\left(1-\frac{T}{T_{c}}\right) \quad \text { near } T_{c} .
$$

La dependencia en temperatura del coeficiente en el momento de (4.106), $\mathcal{M}(T)$, se muestra en la figura 4.21. La parte real crece de manera brusca justo por debajo de la transición de fase. La parte imaginaria se acerca al valor de la fase rota a la temperatura crítica, i.e. $\mathcal{M}\left(T_{c}\right)=-i$, como es de esperarse para que los modos pseudodifusivos se conecten de manera continua con los modos de la fase normal a través de la transición de fase. Nótese que $\operatorname{Im} \mathcal{M}(T)$ decrece a medida que bajamos la temperatura.

Otra forma de corroborar que los modos pseudodifusivos vienen del apareo de los modso difusivos de la fase normal es que su relación de dispersión en la transición de fase se pega. Por lo tanto los dos modos son continuos a través de la transición de fases, sin embargo, en lugar de desarrollar un gap puramente imaginario desarrollan un gap complejo.

El hecho que $\operatorname{Re}(\omega)$ no se anuela para estos modso implica que lo suficientemente cerca de $T_{c}$ y en el límite $k=0$, la respuesta a tiempos tardíos de un estado perturbado va a presentar un decaimiento oscilatorio de las perturbaciones, lo cual significa, a diferencia del caso $U(1)$, que no habrá una temperatura para la cual el comportamiento a tiempos grandes cambie de manera cualitativa. 


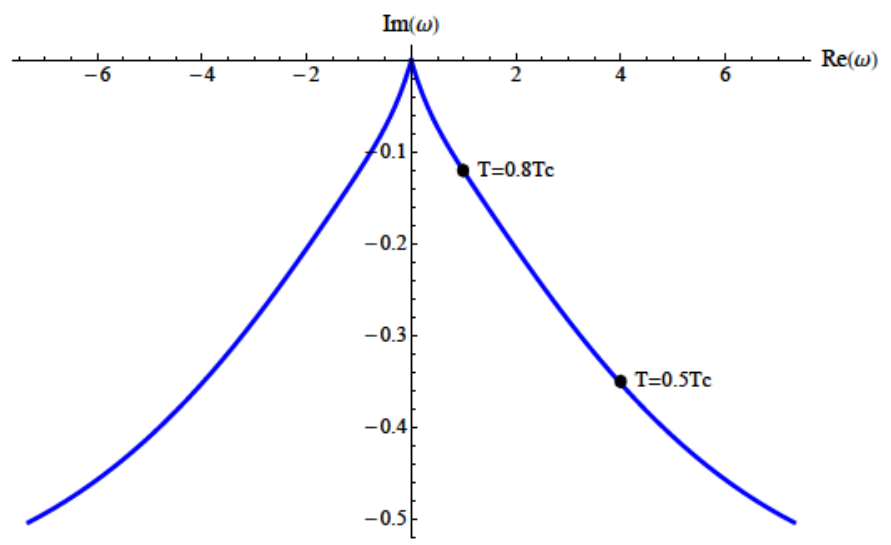

Figura 4.20: $\operatorname{Im} \omega$ versus $\operatorname{Re} \omega$ a $k=0$ como función de la temperatura. La forma de la figura es compatible con simetría de reversión temporal $T$, ya que hay dos modos pseudodifusivos. Tener $\operatorname{Re} \omega(k=0) \neq 0$ es característico del caso no abeliano.
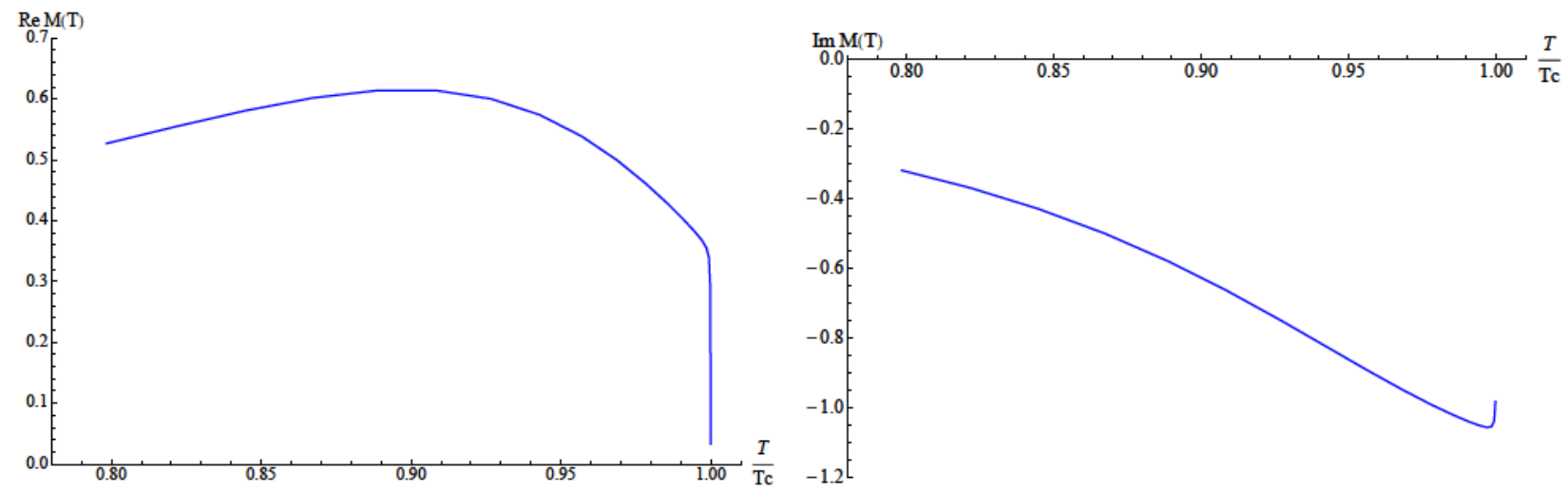

Figura 4.21: Real (left) and imaginary (right) part of $\mathcal{M}(T)$ as a function of $T / T_{c}$. As the temperature approaches $T_{c}$, the value of $\mathcal{M}(T)$ reaches the one prescribed by continuity through the phase transition. 
Modo masivo especial: Buscar a este modo es numéricamente más complicado. Su comportamiento es caracterizado por un gap proporcional a $\mu$. En particular, en [116] se argumentó que un bosón de Goldstone tipo II es acompañado por un modo masivo que satisface $\omega(0)=q \mu$ con $q$ la carga del campo correspondiente. En nuestras convienciones tenemos $q=1$. Así que tenemso que buscar un modo con $\omega(k=0)=\mu$. Más aun, esperamos que se conecte con la componente más baja del escalar complejo conjugado en la fase no rota.

En la figura 4.22 comparamos al modo a momento cero con el consdensado $\bar{\mu}$ en unidades numéricas. Nótese que el modo es continuo en la transición de fase, como era de esperarse. Observamos el comportamiento lineal predicho teóricamente, al menos cerca de $\bar{\mu}_{c}$. Es muy difícil de analizar cuando $\bar{\mu}>6$ debido al alto poder computacional requerido para llevar a cabo el cálculo. El modo muestra una parte imaginaria no nula que se debe a la disipación a temperaturas bajas. Encontramos qu ela parte real por encima de la transición de fase puede ser aproximada por

$$
\operatorname{Re} \omega=1,10 \bar{\mu} \text { near } \bar{\mu}_{c} .
$$

Este resultado muestra una desviación con la conjetura teórica pero podría deberse a fluctuaciones numéricas. Vale enfatizar que el análisis numérico en este caso es bastante desafiante.
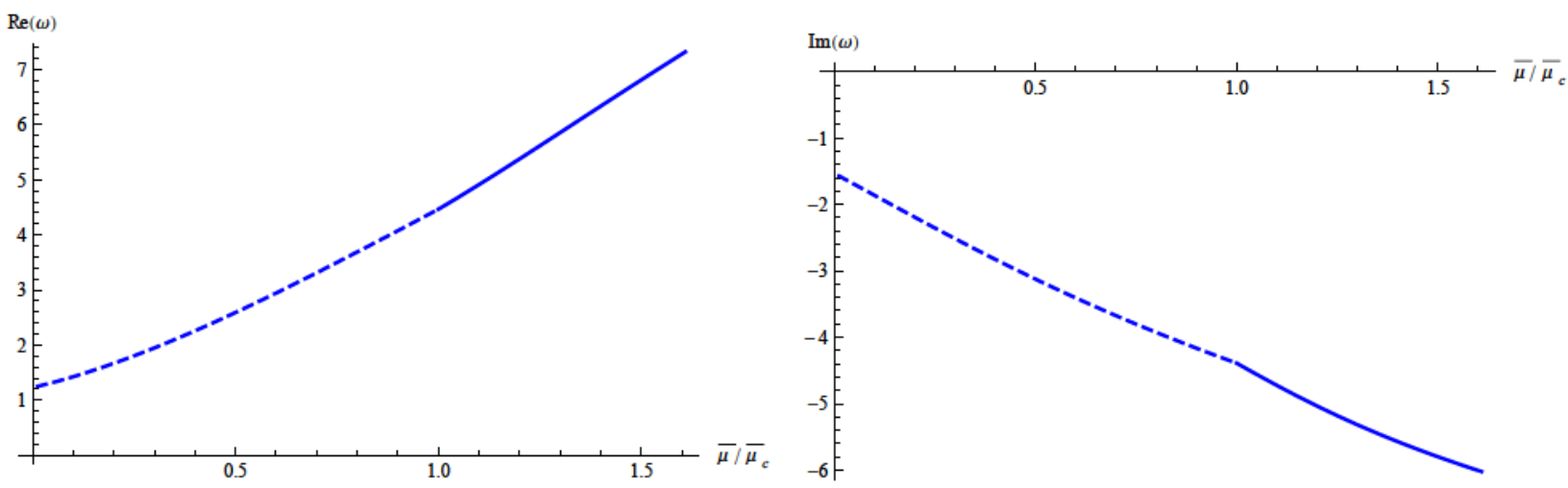

Figura 4.22: Partes real (izquierda) e imaginaria (derecha) del modo masivo especial versus el potencial químico. Encontramos el valor lineal espera con $\mu$. El gráfico cubre tanto la fase normal (línea entrecortada) como la rota (línea sólida).

\subsection{Discusión}

El principal objetivo de este trabajo es mostrar la existencia de modos de Goldstone tipo II en el espectro de QNMs de una teoría holográfica dual a un superfluido fuertemente acoplado con simetría $U(2)$.

Estudiamos dos modelos, uno en el cual sólo la simetría overall $U(1)$ estaba gaugeada en el bulk de AdS y otro en el cual toda la simetría $U(2)$ estaba gaugeada. El descubirmiento más importaantes es que de hecho existen exitaciones no masivas presentes en el espectro de QNMs en el interior de AdS que muestran la relación de dispersión cuadrática esperada, pero de alguna manera inusual, característica de bosones de Goldstone tipo II.

Para el modelo no gaugeado esto constituye un resultado soreprendente. Después de todo, la teoría de campos dual a este modelo no contiene las corrientes conservadas necesarias que corresponderían a los generadores de la simetría $S U(2)$ global. Pruebas tradicionales del 
teorema de Goldstone dan por garantizada su existencia. Por otro lado está casi garantizado que uno puede escribir una teoría de campos efectiva, un modelo onda Landau-Ginzburg simple, que capture la dinámica esencial de los modos livianos, i.e. los modos quasinormales más bajos. Dicho modelo va a estar dado esencialmente por el introducido en la sicción 4.2 y esto garantiza la existencia de los modos de Goldstone tipo II. Sin embargo uno puede esperar que dicho acercamiento a través de teorías de campso efectivas pueda sólo capturar la física de los modos más bajos y no así la de los más altos. Esto es de hecho lo que pasa: el modo compaero del Goldstone tipo II en el modelo ungauged no se comporta de la manera supuestamente universal $\omega=q \mu$. En contraste el modo corresponiente del modelo gaugeadosí obedece esta relación de manera aporximada y la desviación encontrada puede ser perfectamente atribuida a las dificultades numéricas que emergen al estudiar QNMs más altos.

Una perspectiva bastante interesante del modelo ungauge surge si variamos las masas de los campos escalares en el interior de AdS. Si las masas son ligeramente diferentes, entonces a la temperatura crítica sólo uno de los escalares va a tener un QNM no masivo (aquel que tenga una masa más pequeña). el modo escalar más bajo del segundo va a ser todavía masivo a la temperatura crítica. A medida que uno va a través de la transición de fase no esperamos que este modo se vuelva no masivo a temperaturas más bajas. Debería convertirse en un modo pseudo-Goldstone con un gap proporcional a la diferencia de masas. La aparición de un Goldstone tipo II puede ser vista como un efecto del aumento de simetría en ese punto del espacio de parámetros en el que las masas de los escalares se vuelven degeneradas. Como esta simetría no es representada por campos de gauge en el bulk podemos llamarla una simetría accidental. A este punto resulta difícil escapar a la tentación de dibujar un paralelo con el aumento de simetría conjeturado para superconductores de alta $T_{c}$. En [122] fue sugerido que el diagrama de fases de superconductores de alta $T_{c}$ puede ser capturado mediante un agrandamiento de la simetría $S O(3) \times U(1)$ de rotaciones y electromagnetismo a una simetría $S O(5)$. Como los superconductores de alta $T_{c}$ son d-wave en lugar de s-wave queda por ser visto cómo nuestro mecanismo de agrandamento de simetría y los modos de GOldstone tipo II resultantes pueden ser combinados con modelos holográficos de superfluidos tipo d-wave como por ejemplo $[90,91]^{11}$.

El segundo modelo que estudiamos tiene campos de gauge en el bulk para toda la simetría $U(2)$. Hay varias diferencias importantes en comparación con el modelo no gaugeado. La más evidente es que uno puede definir y estudiar el conjunto completo de conductividades correspondientes a la simetría $U(2)$. Nada en especial ocurre en la fase normal, hay simplemente cuatro conductividades asociadas a los cuatro campos de gauge. En la fase rota sin embargo nuevos fenómenos aparecen. En particualar hay conductividades no diagonales distintas de cero. Además hemos encontrado que las conductvidades deiagonales del sector (1) - (2), aquelque contiene el bosón de Goldstone tipo II, tienen polos de función delta a frecuencia cero. En ese sentido este sectro es superconductor. Más aun, ir a una base desacoplada en este sector lleva a un resultado muy sugestivo: la conductividad desarrolla un pico tipo Drude característico de metales además de la delta. Pr otro lado, el criterio de landau para superfluidez no se satisface en este sector. Recordad que el mismo dice que la superfluidez ocurre para velocidades $v$ que son más pequeñas que la velocidad crítica $v_{c}$ donde $v_{c}=\min _{i} \omega_{i}(k) / k$ para todas las ramas de exitación $i$ y para todo momento $k[124,125]$.

\footnotetext{
${ }^{11}$ La aparición de modos de Goldstone no masivos relacionados con un aumento de simetría en el contexto de condensados de Bose fue encontrado en [123]
} 
Para un modo de Goldstone tipo II la velocidad crítica del fluido es claramente nula.

Una segunda diferencia concierne al destino de los modos difusivos. En la fase no rota hay simplemente cuatro modos difusivos, uno para cada campo de gauge en el interior de AdS. En la fase rota hay un modo imaginario puro masivo 'pseudo-diffusivo' en el sector (0) - (3), i.e. en el sector isomorfo al superfluido $U(1)$ s-wave. Como todavía resta una simetría $U(1)$ no rota hay todavía un modo difusivo normal asociado a la simetría $U(1)$ no rota. En el sector $(1)-(2)$ tenemos sin embargo dos modos difusivos en la fase no rota. Al atravesar la transición de fase estos modos pueden aparearse y moverse fuera de los ejes imaginarios, conviritiéndose en un par de modos quasinormales corrientes con partes real e imaginaria en sus frecuencias. Genéricamente la parte imaginaria de este gap es más pequeña (i.e. yace más cerca del eje real) que el gap del modo imaginario puro en el sector (0) - (3) . Una perturbación grande y genérica va, a tiempos grandes, a exitar tanto el sector $(0)-(3)$ como el $(1)-(2)$. La respuesta a tiempos grandes del parámetro de orden invairante frente $U(2)$ $\sqrt{\left|\mathcal{O}_{1}\right|^{2}+\left|\mathcal{O}_{2}\right|^{2}}$ va a estar dominada entonces por estos modos apareados y mostrará un comportamiento oscilatorio en ocntraste con el patrón de respuesta del parámetro de orden en el caso $U(1)$ [102].

Otro QNM remarcable es el modo especial gapeado, i.e. el modo compañero del bosón de Goldstone tipo II. A temperaturas muy altas este modo y aquel que a $T=T_{c}$ se convierte en un mado de sonido son degenerados. A medida que aumentamos la temperatura el gap de estos modos se agranda, para $T<T_{c}$, es de esperarse que $\operatorname{Re}(\omega(k=0))$ para el modo especial gapeado sea proporcional a $q \mu[113,116]$. En particular hemos encontrado $\omega \sim 1,1 \mu$ aunque $q=1$ en nuestras convienciones. Desafortunadamente, con el métdo numérico utilizado encontramos muy difícil al estudio de este modo y la diferencia puede ser una consecuencia de precisión numérica insuficinete. Probablemente valdría la pena estudiar este modo con métodos alternativos como por ejemplo el método de relajación desarrollado en [126, 127].

Hay varias generalizaciones del modelo $U(2)$ que parecen interesantes y que podrían ser estudiadas en el funturo. Una inmediata sería analizar la inestabilidad tipo $p$-wave comentada en la sección 4.4 y buscar un background estable a bajas temperaturas, como se hará en el capítulo 5. Un escenario similar fue encontrado en [161, 129], en donde una versión gaugeada del modelo teórico sigma sufre una transición de fase ocacionada por un condensado vectorial anisotrópico.

Otra posible generalización será analizar el modelo cuando la respuesta de la métrica es tenida en cuenta. Esto introduce al tensor de energía-momento como un operador en la teoría de campos del borde y es de esperarse que los modos usuales de sonido y de cizayadura aparezcan al fluctuar la métrica. Más aun, esto nos permitiría obtrener resultados confiables auna temperaturas muy bajas y por ejemplo calcular la densidad de carga superconductora a temperatura cero, así como $\mathcal{B}(T=0)$.

Hemos ocnstruido acá un modelo simple con bosones de Goldstone tipo II usando una estrategia "bottom-up". Es sin embargo interesante preguntarse si dichos modelos pueden ser realizados mediante una construcción "top-down" derivada de D-branas, teoría de cuerdas o teoría M [130, 131, 132, 133, 134].

Otra posible dirección de investigación involucra usar la correspondencia Fluidos/Gravedad [135] con el fin de derivar la expansión hidrodinámica de la corriente y mediante la inclusión de backreaction también la relación constitutiva para le tensor de energía-momento. Esto esclarecerá el comportamiento de superfluidos no relativistas y es particular debería resultar en la formulación de la hidrodinámica de bosones tipo II rlativistas. Hasta donde sabemos 
esto no es sabido ni a orden dominante, i.e. orden cero en derivadas.

Otra dirección de investigación involucra el criterio de Landau. De acuerdo con este criterio, la relación de dispersión (4.105) previene al sistema de ser apaz de acomodar un superflujo. Más aun, aunque $\sigma_{11}$ y $\sigma_{22}$ son superconductoras, as soon ran pronto como una supercorriente/superflujo se prende el sistema system el sistema debería ser llevado fuera de la fase superfluida inmediatamente. Debe notarse sin embargo que en un superfluido holográfico el condensado y su flujo no son nunca dominantes en una expansión a gran $N$ y el espectro de exitaciones, los QNMs, son subdominantes. Entonces no parece evidente que el criterio de Landau pueda ser aplicado de manera inmediata. Es sabido sin embargo que para un superfluido $U(1)$ existe un superfluido crítico, o una supercorriente crítica, por encima de la cual el condensado se anula [136, 137, 138]. Resulta interesante investigar si es este el caso (a lo [138]) y también estudiar como el mecanismo ocurre. Dicha investigación se presenta en el capítulo 6.

Finalmente resulta interesante preguntarse si modelos holográficos con modos de Goldstone con relaciones de dispersión más altas $\omega=c k^{n}$ with $n>2$ pueden ser construidos. 


\title{
Capítulo 5
}

\section{Superconductores $\mathrm{s}+\mathrm{p}$ holográficos}

\author{
"It's an important and popular fact that things are not always what they seem." \\ The Hitchhikers Guide to the Galaxy.
}

En este capítulo estudiamos el diagrama de fases del modelo holográfico con una simetría $U(2)$ global en el borde presentado en el capítulo 4 y mostramos que a bajas temperaturas una fase con condensados tanto escalar $s$ como vector $p$ existen. Esta es la fase $s+p$-wave donde la simetría global $U(2)$ y también la simetría de rotaciones están rotas. Estudiando la energía libre mostramos que esta fase es la preferida cuando existe. También consideramos configuraciones desbalanceadas donde un segundo potencial químico es encendido. Éstas presentan un diagrama de fases rico caracterizado por la competencia y la coexistencia de parámetros de orden $s$ y $p$. Los resultados presentados en este capítulo vieron la luz por vez primera en [140].

\subsection{Introducción}

Un problema interesante en la arena de superfluidos y superconductores no convencionales es aquel de la competencia y coexistencia de distintos parámetros de orden [122]. Un ejemplo paradigmático en el reino de los superfluidos es el del ${ }^{3} \mathrm{He}$. A bajas temperaturas el ${ }^{3} \mathrm{He}$ presenta dos fases superfluidas distintas, Llamadas fases $A$ y $B$ [141] por motivos históricos. ${ }^{3} \mathrm{He}-B$ es la fase a temperaturas bajas (y presiones bajas) y corresponde a superfluido $p$ wave, donde el parámetro de orden transforma como un vector bajo rotaciones espaciales. ${ }^{3} \mathrm{He}-A$ es la fase superfluida a temperaturas altas (y presiones altas). Es un superfluido chiral $p$-wave cuyo parámetro de orden es un vector complejo, y las simetrías de reversión temporal y paridad están rotas de manera espontánea. La figura 5.1 muestra el diagrama de fases para el ${ }^{3} \mathrm{He}$. En el dominio de superconductores no convencionales has sido mostrado en [142] semiconductores de gap angosto tridimensionales dopados como por ejemplo $\mathrm{Cu}_{x} \mathrm{Bi}_{2} \mathrm{Se}_{3} \mathrm{o}$ $\mathrm{Sn}_{1-x} \operatorname{In}_{x}$ Te hay una competencia entre estados superconductores $s$ y $p$-wave. Moviendo las constantes de acoplamiento de los dos canales (correspondientes a apareamientos tipo $s$ y $p$ ) lleva a un diagrama de fases en el que tanto la fase $p$ como $s$-wave existen. Más aun, donde las dos fases se solapan un nuevo estado $p+i s$ aparece. El parámetro de orden para esta fase es la combinación de un vector y de un pseudoescalar, y rompe las simetrías de reversión temporal 


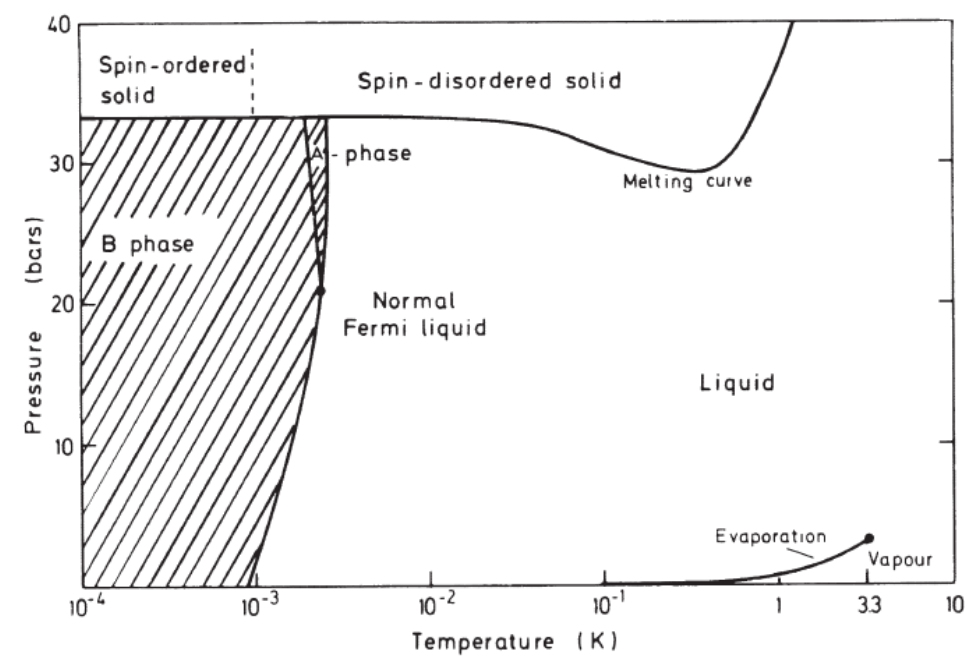

Figura 5.1: Diagrama de fases de presión contra temperatura para el ${ }^{3} \mathrm{He}$.

y paridad haciendo de este estado un ejemplo interesante de superconductor topológico ${ }^{1}$.

La correspondencia AdS/CFT fue exitosa en la construcción de versiones holográfica de superconductividad [?, 27] (para mayor información [1, 92]). Más aun, modelos holográficos de estados superconductores $s$ [28], $p$ [29] y $d$-wave [144]; os cuales tienen parámetros de orden escalar, vector y de spin-2 respectivamente, fueron desarrollados en los últimos años. La coexistencia y competencia de varios parámetros de orden también fue estudiada holográficamente en [145, 146, 147, 148, 149, 150, 151, 152].

En este capítulo, trabajando sobre el modelo construido en [89] y desarrollado en el capítulo 4, desarrollamos el dual holográfico de un superconductor con condensados $s$-wave y $p$-wave. Luego estudiamos el diagrama de fases de mezclas desbalanceadas (en las que dos potenciales químicos fueron encendidos) encontrando una competencia entre las fases superconductoras $s, p$, y $s+p$-wave.

En [89] el dual holográfico de un superfluido de dos componentes fue construido, usando un doblete escalar conviviendo con un campo de gauge de $U(2)$ en la geometría de un agujero negro $(\mathrm{BH})$ de Schwarzschild plano. Al prender un potencial químico en la componente overall $U(1) \subset U(2)$, el sistema se hace inestable hacia la condensación del doblete escalar. La aparición del condensado escalar rompe de manera espontánea la simetría $U(2)$ dejando apenas un $U(1)$, lo que señala una transición de fases a un superfluido $s$-wave. En esta fase dos densidades de carga distintas están presentes, correspondientes a los dos $U(1)$ s dentro de $U(2)$, por lo tanto llevando a cabo la realización holográfica de un superfluido de dos componentes. Fue también encontrado que la fase superfluida $s$-wave es aún inestable hacia la condensación de un modo vectorial, que rompe el $U(1)$ restante y da lugar a una fase con dos condensados: el superconductor holográfico $s+p$-wave. Acá estudiamos dicha fase

\footnotetext{
${ }^{1}$ Este es de hecho un ejemplo de un estado axiónico de la materia. Esta fase $p+i s$ pertenece a la clase D en la clasificación [143] de superconductores topológicos tridimensionales. Posee fermiones de Majorana gapeados como estados de borde lo cual da lugar a un efecto Hall termal de superficie anómalo. Sería interesante realizar holográficamente este estado superconductor axiónico (ver [146] para un superconductor holográfico con rotura de simetría de reversión temporal $p+i p)$.
} 
y establecemos el diagrama de fases del superfluido de dos componentes. Si uno trabaja en el ensamble gran canónico, en el que el potencial químico de la teoría del borde está fijo, la temperatura del sistema viene dada por $T \propto 1 / \mu$, donde $\mu$ es un potencial químico adimensional relacionado con aquel de la teórica del borde mediante reescaleos. La imagen final es la siguiente: a potencial químico $\mu$ lo suficientemente pequeño (temperaturas altas) el sistema se encuentra en una fase normal en la que no hay ningún condensado encendido. Para $\mu$ mayor que un cierto valor crítico $\mu_{s}$ el campo escalar adquiere un valor de expectación y el sistema entra en la fase superfluida $s$-wave. Yendo a potenciales químicos aún más grandes una nueva transición de fases ocurre: a $\mu_{s p}>\mu_{s}$ un condensado vectorial aparece para $\mu>\mu_{s p}$ y el sistema está en una fase $s+p$-wave con parámetros de orden escalar y vectorial no nulos.

También consideramos el caso en el que los dos potenciales químicos correspondientes a los dos $U(1) \mathrm{s} \subset U(2)$ están prendidos. Este setup, donde el $U(2)$ está explícitamente roto en $U(1) \times U(1)$, realiza una mezcla desbalanceada, caracterizada por la existencia de dos especies de carga caracterizadas por dos potenciales químicos. Ejemplos de dichos sistemas son las mezclas de fermiones desbalanceadas [153], y QCD a potencial bariónico y de isospin finito [154]. Más aún, superconductores desbalanceados son sistemas interesantes donde es de esperarse la aparición de fases anisotrópicas e inhomogéneas [155, 156]. Realizaciones holográficas de superconductores desbalanceados fueron construidas en [157, 158]. Para nuestro sistema determinamos el diagrama de fases como función de los dos potenciales químicos y encontramos que existen fases $s$-wave, $p$-wave and $s+p$-wave.

\subsection{El superfluido holográfico de dos componentes}

Consideremos ahora el dual holográfico de un fluido multicomponente consistente en un doblete escalar cargado bajo campos de gauge $U(2)$ que vive en la geometría de brana negra de Schwarzschild-AdS $3+1$ dimensional construida en $[160]^{2}$. La acción de dicho sistema es

$$
S=\int d^{4} x \sqrt{-g}\left(-\frac{1}{4} F_{c}^{\mu \nu} F_{\mu \nu}^{c}-m^{2} \Psi^{\dagger} \Psi-\left(D^{\mu} \Psi\right)^{\dagger} D_{\mu} \Psi\right)
$$

with

$$
\begin{aligned}
& \Psi=\sqrt{2}\left(\begin{array}{c}
\lambda \\
\psi
\end{array}\right), \quad D_{\mu}=\partial_{\mu}-i A_{\mu}, \quad A_{\mu}=A_{\mu}^{c} T_{c}, \\
& T_{0}=\frac{1}{2} \mathbb{I}, \quad T_{i}=\frac{1}{2} \sigma_{i} .
\end{aligned}
$$

El sistema vive en un background de Schwarzschild-AdS

$$
\begin{aligned}
& d s^{2}=-f(r) d t^{2}+\frac{d r^{2}}{f(r)}+r^{2}\left(d x^{2}+d y^{2}\right) \\
& f(r)=r^{2}\left(1-\frac{1}{r^{3}}\right)
\end{aligned}
$$

\footnotetext{
${ }^{2}$ Un modelo similar fue introducido en [159] con el fin de describir superconductores holográficos multibanda.
} 
donde hemos fijado el radio de AdS y del horizonte a $L=r_{h}=1$, usando las simetrías de escaleo del sistema. Trabajamos en el límite de desacoplo, en el cual la backreaction de los campos de materia en la métrica es despreciada.

Consideramos el siguiente ansatz (consistente) para los campos en nuestro setup [89]

$$
A_{0}^{(0)}=\Phi(r), \quad A_{0}^{(3)}=\Theta(r), \quad A_{1}^{(1)}=w(r), \quad \psi=\psi(r),
$$

donde todas las funciones son reales. Todos los otros campos en (5.1) son fijados a cero, en particular fijamos $\lambda=0$ sin pérdida de generalidad. Las ecuaciones de movimiento resultantes son

$$
\begin{aligned}
& \psi^{\prime \prime}+\left(\frac{f^{\prime}}{f}+\frac{2}{r}\right) \psi^{\prime}+\left(\frac{(\Phi-\Theta)^{2}}{4 f^{2}}-\frac{m^{2}}{f}-\frac{w^{2}}{4 r^{2} f}\right) \psi=0, \\
& \Phi^{\prime \prime}+\frac{2}{r} \Phi^{\prime}-\frac{\psi^{2}}{f}(\Phi-\Theta)=0, \\
& \Theta^{\prime \prime}+\frac{2}{r} \Theta^{\prime}+\frac{\psi^{2}}{f}(\Phi-\Theta)-\frac{w^{2}}{r^{2} f} \Theta=0, \\
& w^{\prime \prime}+\frac{f^{\prime}}{f} w^{\prime}+\frac{\Theta^{2}}{f^{2}} w-\frac{\psi^{2}}{f} w=0 .
\end{aligned}
$$

En lo que sigue vamos a considerar que el escalar tiene $m^{2}=-2$ y que el operador dual correspondiente tenga dimensión 2.

El comportamiento asintótico UV de los campos, correspondiente a soluciones de las ecuaciones $(5.6$ - 5.9) en el límite $r \rightarrow \infty$, viene dado por

$$
\begin{aligned}
& \Phi=\mu-\rho / r+O\left(r^{-2}\right), \\
& \Theta=\mu_{3}-\rho_{3} / r+O\left(r^{-2}\right), \\
& w=w^{(0)}+w^{(1)} / r+O\left(r^{-2}\right), \\
& \psi=\psi^{(1)} / r+\psi^{(2)} / r^{2}+O\left(r^{-3}\right),
\end{aligned}
$$

donde, del lado dual, $\mu$ y $\rho$ son respectivamente el potencial químico y la densidad de carga correspondientes al overall $U(1) \subset U(2)$ generado por $T_{0}$, mientras $\mu_{3}$ y $\rho_{3}$ son el potencial químico y la densidad de carga correspondientes al $U(1) \subset S U(2)$ generado por $T_{3}$. $\psi^{(1)}$ es la fuente del operador escalar de dimensión 2 , mientras que $\psi^{(2)}$ es su valor de expectación. Finalmente $w^{(0)}$ y $w^{(1)}$ son la fuente y vev de un operador corriente $J_{x}^{(1)}$ (recuérdese que $A_{\mu}^{(1)}$ es dual a la corriente $\left.J_{\mu}^{(1)}\right)$. Nótese que en un background donde $w(r)$ condensa el $S U(2) \subset U(2)$ es roto de manera espontánea, y más aún la simetría de rotaciones espaciales está rota también.

\subsection{El superconductor holográfico $\mathrm{s}+\mathrm{p}$-wave}

Estamos buscando soluciones de las ecuaciones (5.6 - 5.9) donde $\psi, w$, o ambos adquieren perfiles no triviales. Queremos que los mismos realicen una rotura espontánea de simetría así que imponemos que las contribuciones dominantes en el UV (duales a las fuentes de los operadores correspondientes) se anulen. Vamos a prender el potencial químico $\mu$ a lo largo 
del overall $U(1)$, mientras que pediremos que el otro potencial químico $\mu_{3}$ permanezca nulo. Por lo tanto nuestras condiciones de borde en el UV son

$$
\psi^{(1)}=0, \quad w^{(0)}=0, \quad \mu_{3}=0 .
$$

En el IR, por regularidad debemos pedir que $A_{t}$ se anule en el horizonte del BH.

Nótese que después de usar las simetrías de escaleo del sistema para fijar los parámetros del agujero negro en (5.4), la única escala del problema viene dada por el potencial químico $\mu$. En el ensamble gran canónico, en el que el potencial químico es fijado, la temperatura es inversamente proporcional al potencial químico reescaleado $T \propto 1 / \mu$. Entonces, variar $\mu$ es equivalente a cambiar la temperatura del sistema. Por ese motivo, los resultados de este capítulo están presentados en términos de $\mu$.

Hemos buscado soluciones numéricas con $\psi$ y $w$ no cero, integrando desde el IR hacia el UV donde imponemos las condiciones de borde (5.14). Hemos encontrado las siguientes soluciones:

Fase normal: para todos los valores de $\mu$ existe una solución analítica en la que $\psi=w=$ $\Theta=0$ y $\Phi=\mu(1-1 / r)$. Esta solución describe el estado normal del sistema, donde la simetría $U(2)$ vive ininmutable.

Fase $s$-wave: para $\mu \geq \mu_{s} \approx 8,127$ encontramos soluciones con $\psi$ no nulo. Como fue observado en [89] para estas soluciones las ecuaciones se desacoplan en dos sectores: uno correspondiente al superconductor holográfico abeliano [27] y otro correspondiente a la simetría $U(1)$ sin romper. Aunque $\mu_{3}$ es cero como fue requerido en (5.14), las dos densidades de carga $\rho$ y $\rho_{3}$ son no nulas y por lo tanto un superfluido de dos componentes es realizado. Fase $s+p$-wave: para $\mu \geq \mu_{s p} \approx 20,56$ hay soluciones que satisfacen (5.14) con $\psi$ y $w$ no cero. En estas soluciones la simetría $U(2)$ está completamente rota, y más aún, como $w^{(1)} \sim\left\langle J_{x}^{(1)}\right\rangle$ la simetría de rotaciones espaciales está rota también. De nuevo $\mu_{3}=0$ mientras que $\rho$ y $\rho_{3}$ son distintas de cero, por lo que realizan una fase $s+p$-wave de un superfluido con dos componentes. Usualmente la superconductividad tipo $p$-wave es disparada por un potencial químico $\mu_{3}$ [29]. En nuestro caso la componente $p$ del superfluido $s+p$ es mantenida por la densidad de carga espontáneamente inducida $\rho_{3}$. Por ese motivo no vemos soluciones donde sólo el condensado $p$ esté presente en este sistema.

En la figura 5.2 graficamos los condensados $\left\langle O_{2}\right\rangle \sim \psi^{(2)}$ y $\left\langle J_{x}^{(1)}\right\rangle \sim w^{(1)}$ como función del potencial químico. Nótese que la solución en la que ambos condensados coexisten se extiende hasta el $1 / \mu$ más baja (o equivalentemente la temperatura más baja) para la cual podemos confía en el límite de desacoplamiento y por lo tanto nos podemos permitir ignorar el efecto en la métrica.

Para determinar le diagrama de fases de nuestro sistema computamos la energía libre para las diferentes soluciones y establecemos cual es la preferida cuando más de una solución existe. La densidad de energía libre viene dada por la acción on-shell, y para nuestro ansatz es

$$
\begin{aligned}
F & =-\frac{T}{V} S_{E}=-\frac{1}{2}\left(\mu \rho+\mu_{3} \rho_{3}\right)+ \\
& +\int \frac{d r}{2 f}\left(-f w^{2} \psi^{2}+r^{2}(\Phi-\Theta)^{2} \psi^{2}+\frac{f}{r^{2}} w^{2} \Theta^{2}\right) .
\end{aligned}
$$

La energía libre para las distintas soluciones se muestra en la figura 5.3. A potencial químico finito sólo la fase normal existe. A $\mu=\mu_{s} \approx 8,127$ hay una transición de fases de segundo 


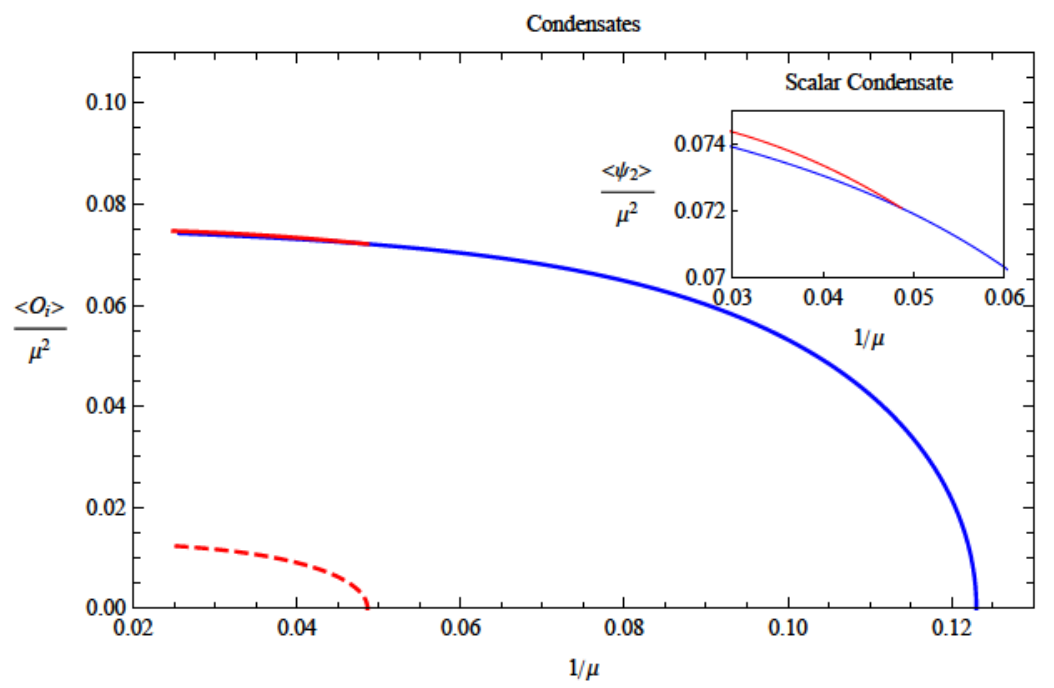

Figura 5.2: Condensados $\psi^{(2)}$ (continuo) y $w^{(1)}$ (entrecortado) como función de $1 / \mu$ en las fases $s$-wave (azul) y $s+p$-wave (rojo). El condensado $p$ aparece a un $\mu_{s p}$ tal que $\mu_{s} / \mu_{s p}=$ 0,395 como fue encontrado en [89]. El gráfico incrustado hace zoom a $\psi^{(2)}$ para mostrar la diferencia en el condensado escalar para las soluciones $s$ (azul) y $s+p$ (rojo).

orden a una solución $s$-wave. Si uno sigue aumentando $\mu$, a $\mu_{s p} \approx 20,56$ hay una segunda transición de fases de segundo orden desde las fase $s$-wave hacia la fase $s+p$-wave. El sistema se queda en la fase $s+p$-wave para $\mu>\mu_{s p}$.

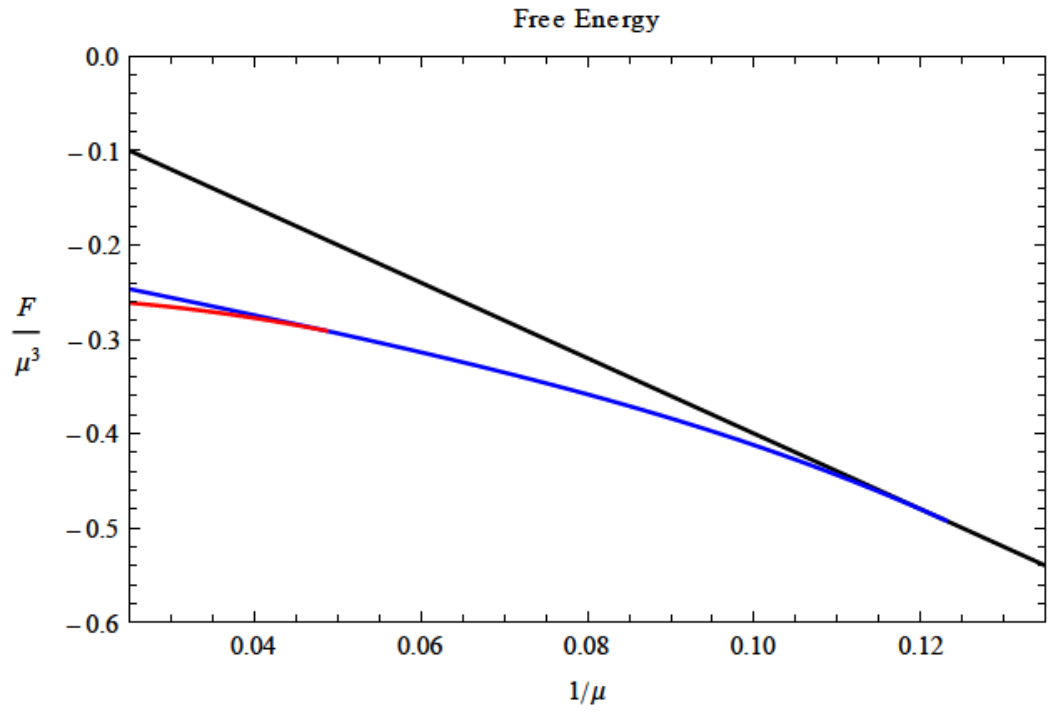

Figura 5.3: Energía libre de diferentes soluciones como función de $1 / \mu$ : la fase normal está de negro, la fase $s$-wave en azul, y la $s+p$-wave de rojo. 


\subsection{Superconductores desbalanceados}

En esta sección relajamos la condición $\mu_{3}=0$ y estudiamos el diagrama de fases del sistema como función de $\mu \mathrm{y} \mu_{3} / \mu$. Nótese que prender un segundo potencial químico significa romper explícitamente $U(2) \rightarrow U(1) \times U(1)$. El sistema puede ahora ser interpretado como el dual holográfico de una mezcla desbalanceada [158, 157].

Ahora que el $U(2)$ está roto de manera explícita, no podemos imponer genéricamente que $\lambda=0$ mediante el uso de las transformaciones de gauge. Entonces, en principio ambas componentes del doblete escalar podrías condensar. En [159] fue estudiado que opción es termodinámicamente favorable. Siguiendo su análisis, el elegir que el condensado ocurra para la componente más baja nos fuerza a fijar $\mu_{3} / \mu<0$ para que las soluciones sean estables.

La condiciones de contorno en el UV serán ahora

$$
\psi^{(1)}=0, \quad w^{(0)}=0 .
$$

Como antes, usamos métodos numéricos para integrar el sistema (5.6 - 5.9). Nos encontramos en un escenario donde cuatro soluciones distintas existen:

Fase normal: una solución analítica tal que $\psi=w=0, \Phi=\mu(1-1 / r)$ y $\Theta=\mu_{3}(1-1 / r)$ existe para cualquier valor de $\mu$ y $\mu_{3}$, y describe el estado normal del sistema.

Fase $s$-wave: para $\mu-\mu_{3} \geq 8,127$ encontramos soluciones con $\psi$ no nulon que nos recuerdan a aquellas del caso balanceado.

Fase $p$-wave: para $\left|\mu_{3}\right| / \mu \geq 3,65 / \mu$ existen soluciones que satisfacen $\psi=0$, pero $w \neq 0$ y als condiciones de borde adecuadas (5.16). El condensado escalar $\left\langle O_{2}\right\rangle$ es nulo mientras que $\left\langle J_{x}^{(1)}\right\rangle \neq 0$. Estas soluciones rompen $U(1) \times U(1)$ a $U(1)$ y también rompen el $S O(2)$ correspondiente a rotaciones espaciales. Nótese que $w(r)$ no está cargado bajo el overall $U(1)$ y por lo tanto esta solución es insensible al valor de $\mu$. Esta situación cambiría si la backreaction de lso campos de materia en la métrica fuese tomada en cuenta, como en $[157,158]$.

Fase $s+p$-wave: para valores pequeños de $\mu_{3} / \mu$ encontramos la extensión de la solución $s+p$-wave encontrada en la sección anterior a $\mu_{3}=0$. Sin embargo, mientras más grande sea $\left|\mu_{3}\right| / \mu$ más grande será el $\mu$ al cual la fase aparezca. También encontramos soluciones con dos condensados en una región intermedia en la cual $\mu_{3}$ es grande y $\mu$ está cera del valor crítico $\mu_{s}$. Sin embargo éstas son siempre energéticamente desfavorecidas en comparación a soluciones $s$-wave (ver la figura 5.4).

Computando la energía libre (5.15) de diferentes soluciones determinamos el diagrama de fases para el sistema como función de $1 / \mu$ y $\mu_{3} / \mu$ el cual graficamos en la figura 5.4. Para valores pequeños de $\mu_{3} / \mu$ la situación es muy parecida a aquella encontrada en al sección anterior para $\mu_{3}=0$. Como fue ya mencionado, a medida que $\left|\mu_{3}\right| / \mu$ se hace más y más grande, la transición a la fase $s+p$-wave ocurre para valores más altos de $\mu$. Podría suceder que la fase eventualmente desaparezca a un valor finito de ese cociente, pero esto ocurriría en una región en la que le límite de prueba no es válido, y la backreaction debería se tenida en cuenta ${ }^{3}$. Para $\left|\mu_{3}\right| / \mu$ lo suficientemente grande, la fase $p$-wave es preferida a valores intermedios de $\mu$. Entonces, a medida que $\mu$ es incrementado por encima de un valor

\footnotetext{
${ }^{3}$ Nótese que si la fase $s+p$-wave sobreviviese hasta $1 / \mu=0$ para $\mu_{3} / \mu$ más bajo que cierto valor crítico (como el diagrama de fases 5.4 parece implicar) estaríamos en presencia de un punto crítico cuántico en el cual el sistema va de una fase $s+p$ a una fase $s$-wave. Esto nos recuerda a lo que pasa en [142] para el superconductor $p+i s$.
} 
crítico $\mu_{p}$ el sistema va de la fase normal a la fase $p$-wave phase a través de una transición de fases de segundo orden. Si $\mu$ es incrementado más aún una transición de fases de primer orden lleva al sistema de un estado $p$-wave hacia un estado $s$-wave. Esta transición de fases de primer orden $p$ - a $s$-wave first order phase transition es ilustrada en la figura 5.5 donde dibujamos la energía libre en ambas fases (y aquella correspondiente a la fase normal) como función de $\mu$ para un valor fijo de $\mu_{3} / \mu=-1$. El punto tricrítico donde las fases normal, $s$-wave y $p$-wave se encuentran ocurre a $1 / \mu \approx 0,223$ y $\left|\mu_{3}\right| / \mu \approx 0,815$. La solución $p$-wave nunca es energéticamente preferida para $\left|\mu_{3}\right| / \mu<0,815$.

Un comentario de precaución sobre el diagrama de fases de la figura 5.4: En las regiones del espacio de parámetros donde $\left|\mu_{3}\right| / \mu \gg 1$ o $1 / \mu \ll 1$ el límite de prueba no es válido, $\mathrm{y}$ por lo tanto el diagrama de fases podría verse modificado una vez que la backreaction sea tenida en cuenta. De hecho, la naturaleza de las diferentes transiciones de fase, así como los valores críticos de los potenciales químicos podrían verse alteradas en dichas regiones. Sin embargo, las principales características del diagrama de fases como la existencia de fases $s$ y $p$-wave encontrándose en un punto tricrítico deberían sobrevivir niveles de backreaction moderados.

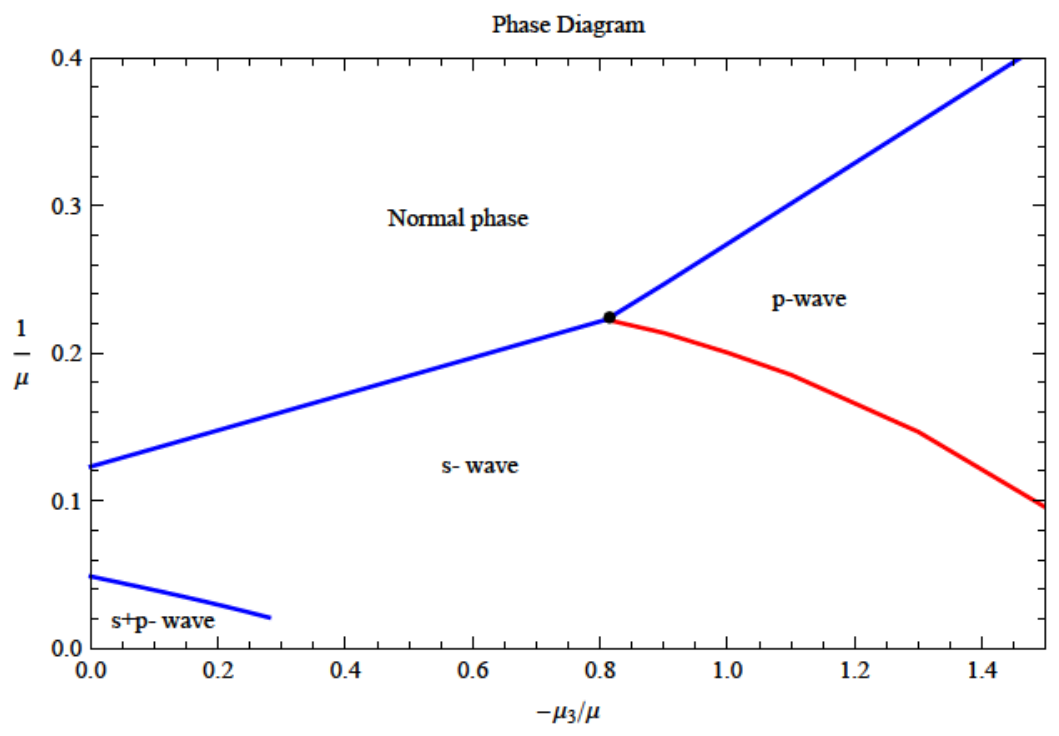

Figura 5.4: Diagrama de fases para sistemas desbalanceados como función de $1 / \mu$ y $\mu_{3} / \mu$. Las transiciones de fases de segundo orden son marcadas con líneas azules,mientras que la línea roja corresponde a una transición de fases de primer orden.

\subsection{Conclusiones}

En este capítulo reportamos la construcción de un estado superconductor $s+p$-wave. Esta fase, en la que tanto los condensados $s$-wave como $p$-wave existen, es el estado preferido del superfluido holográfico de dos componentes presentado en la sección 4. Este modelo realiza una simetría $U(2)$ global en la teoría del borde y presenta estados superconductores con densidades de carga no nulas correspondientes a los dos $U(1)$ s dentro de $U(2)$. 


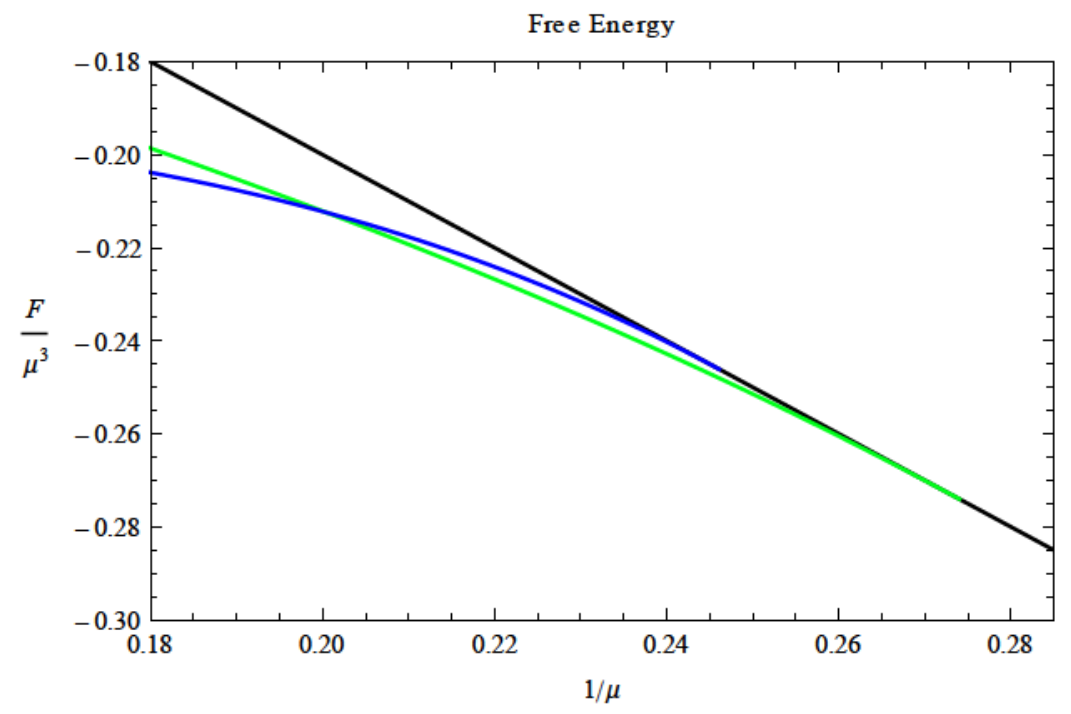

Figura 5.5: La energía libre como función de $1 / \mu$ para $\mu_{3} / \mu=-1$. El color negro corresponde con la fase normal, el azul a la fase $s$-wave, y el verde a la fase $p$-wave.

Los resultados principales se pueden resumir en las figuras 5.2 y 5.4. La figura 5.2 muestra que un estado $s+p$-wave aparece a bajas temperaturas. El análisis de la energía libre determina que el sistema entra a esta fase mediante una transición de fases de segundo orden, y permanece en la misma hasta temperaturas tan bajas como logramos conseguir. Por otro lado, la figura 5.4 presenta el diagrama de fases para el sistema desbalanceado: los potenciales químicos asociados a ambos $U(1) \mathrm{s} \subset U(2)$ son encendidos, y por lo tanto $U(2)$ es roto de manera explícita a $U(1) \times U(1)$. En este diagrama de fases tres fases superconductoras están presentes. Estas son la fase $s$-wave estándar donde un condensado escalar rompe el $U(1) \times U(1)$ en $U(1)$; una fase $p$-wave donde $\left\langle J_{x}^{(1)}\right\rangle \neq 0, U(1) \times U(1)$ es roto a un $U(1)$ (diferente), y también la simetría rotacional es rota; y una fase $s+p$-wave donde $U(1) \times U(1)$ está completamente roto y condensados $s$ y $p$-wave aparecen, y de nuevo la simetría rotacional está rota. Notablemente, mientras el sistema va de la fase normal a las fases $s$-wave and $p$-wave a través de una transición de fases de segundo orden, la transición de fases entre las fases $s$ y $p$-wave es siempre de primer orden. La existencia de esta transición de fases de primer orden entre estados superconductores en un sistema desbalanceado es una predicción interesante de nuestro modelo holográfico. Estas conclusiones podrían ser sensibles a la backreaction ya que, como fue mencionado, el orden de las transiciones de fase puede cambiar cuando la dinámica de la métrica entra en juego. Más aún, en orden de asegurarse la estabilidad de estas fases es importante estudiar el espectro de modos quasinormales del sistema. Como fue señalado en [160], puede ocurrir que inestabilidades hacia fases inhomogéneas aparezcan.

En [161] un modelo de QFT con una simetría $U(2)$ y con un esquema de rotura de simetría parecido al nuestro es estudiado. Los autores encuentran en el espectro de excitaciones un rotón en la dirección del condensado vectorial. Sería interesante estudiar el espectro de QNMs de la fase $s+p$-wave y ver si algo parecido ocurre en nuestro caso. Dejamos este aspecto para investigaciones futuras. 


\title{
Capítulo 6
}

\section{Superfluidos holográficos y el criterio de Landau}

\author{
"Bravo furbo, la risposta è sottile, Maltese."
}

Il Monaco

En este capítulo vamos a abocarnos a la estabilidad de superfluidos holográficos a velocidad finita. Nuestro método se basa en aplicar el criterio de Landau al espectro de modos quasinormales (QNM). En particular estudiamos los modos quasinormales relacionados con los modos de Goldstone asociados a la rotura de simetría con relaciones de dispersión lineales y cuadráticas. En el caso lineal mostramos que la velocidad del sonido se hace cero para velocidad del fluido lo suficientemente grande y que la parte imaginaria de modo quasinormal se mueve para el semiplano superior. Como la inestabilidad tiene un pico a momento finito, lo tomamos como indicación de una fase inhomogénea o con bandas para velocidades del superfluido lo suficientemente grandes. En el caso con relación de dispersión cuadrática la inestabilidad aparece para velocidades arbitrariamente pequeñas a todas temperaturas. Los resultados de este capítulo fueron presentados en [160].

\subsection{Introducción}

La propiedad característica de un superfluido es su habilidad de fluir sin sufrir fricción a través de capilares delgados. Es útil pensar a un superfluido como un líquido de dos componentes. Una componente es el vacío con un número de ocupación macroscópico y la otra es la componente normal, sujeta a fricción y viscosidad. A temperaturas muy bajas la componente normal puede ser descrita como un gas de excitaciones quasipartículas por encima de un estado de vacío ocupado macroscópicamente. Un famoso argumento de Landau [124, 162, 163] fija un límite a la velocidad del fluido que el condensado puede desarrollar. La esencia del argumento es la siguiente. A temperatura cero, la energía de una quasipartícula con momento $\vec{p}$ es $\epsilon(\vec{p})$ en el marco de referencia de reposo para el condensado. Si imaginamos una situación en la que el condensado se mueve con velocidad constante $\vec{v}$ el costo de energía para crear una quasipartícula es

$$
\epsilon^{\prime}(\vec{p})=\epsilon(\vec{p})+\vec{v} \cdot \vec{p}
$$


En particular si $\vec{p}$ es antiparalela a la velocidad del flujo $\vec{v}$ esta energía se ve disminuida y eventualmente va a cero. Si $\epsilon^{\prime}<0$ es energéticamente favorable para le sistema crear excitaciones elementales y poblar estados con esta energía efectiva negativa. Como la velocidad del superfluido $\vec{v}$ es mantenida constante esto significa que el condensado eventualmente va a quedar completamente saqueado y el superflujo se detiene. Se sigue que existe una velocidad de flujo crítica por encima de la cual el superfluido deja de existir. El famoso criterio de Landau para la existencia de superflujo es entonces

$$
v_{\max }=\min \frac{\epsilon(p)}{p},
$$

donde el mínimo sobre todas las ramas de excitaciones elementales debe ser tomado. Se sabe por ejemplo que para el Helio superfluido que la componente normal a bajas temperaturas puede ser descrita de manera precisa como un gas de fonones y rotones y que la velocidad crítica no está determinada por el mínimo de las relaciones de dispersión de fonones y rotones sino por excitaciones de vórtices, lo que resulta en una velocidad de superfluido máxima mucho más baja.

A temperatura finita suele haber una componente de fluido normal presente y por lo tanto la energía de una excitación de un superfluido con superflujo no puede ser contenido simplemente mediante un boost (Galileano) como en la ecuación (6.1). Es sin embargo todavía cierto que la energía va a depender de la velocidad del superfluido y que puede volverse negativa si la velocidad del superfluido es lo suficientemente grande. A temperatura finita el criterio es por lo tanto que el superfluido será estable siempre y cuando todas las excitaciones de quasipartícula tengan energía positiva. Si en un superfluido las únicas excitaciones de energía baja son los fonones este criterio implica básicamente que la velocidad del sonido, que dependerá del superflujo, sea positiva en todas las direcciones.

En $[136,137]$ un superfluido s-wave en $2+1$ dimensiones con superflujo fue construido y fue señalado que hay de hecho una velocidad crítica por encima de la cual el estado superfluido deja de existir. El diagrama de fases obtenido en estos trabajos está basado en comparar la energía libre del superflujo con la energía libre de la fase normal. Resulta ser que la transición de fases de la fase superfluida a la fase normal es de primer o segundo orden dependiendo de la temperatura. Un hecho interesante es que en $3+1$ dimensiones hay un rango de masas para el condensado para las cuales la transición de fases es de segundo orden [164]. Otra forma de establecer el diagrama de fases fue usada en [138]. Allí la supercorriente fue mantenida fija y se argumenta que la transición de fases es siempre de primer orden.

El significado físico de comparar la energía libre de un estado con superflujo y un estado normal no es del todo clara, ya que para todas las temperaturas por debajo de la crítica le estado normal es inestable hacia la condensación de un superfluido sin superflujo. De hecho el superflujo por sí mismo es un estados metaestable [163] como fue enfatizado ya en [136]. Vamos a proponer un método diferente para caracterizar el diagrama de fases de manera que quede relacionado de manera más directa con el criterio de estabilidad (6.2).

El propósito de este capítulo es entonces estudiar cómo se realiza el criterio de estabilidad (6.2) en superfluidos holográficos. Los modelos holográficos más simples de superfluidos son obtenidos en el límite de desacoplamiento. En este límite se descartan las fluctuaciones de la métrica y uno se queda solamente con la dinámica del escalar cargado y el campo de gauge en un agujero negro asintóticamente AdS. El espectro de excitaciones de una teoría de campos holográfica a temperatura y densidad de carga finitas viene dada por el espectro 
de modos quasinormales (QNMs) [96, 97, 98, 99]. Los QNMs del modelo holográfico más simple con una simetría $U(1)$ rota de manera espontánea fue obtenido en [100]. Este modelo fue generalizado para una simetría $U(2)$ [89] como vimos en el capítulo $4^{1}$, lo que da lugar al dual holográfico de un fluido multicomponente [103]. El espectro del modelo $U(2)$ resulta contener una copia del espectro usual de QNM del modelo $U(1)$ pero también una nueva característica, la aparición de un bosón de Goldstone tipo II.

Es dominio público que la rotura espontánea de una simetría global continua lleva a la aparición de modos no masivos, los bosones de Goldstone. Esto es también respetado por teorías holográficas. Los bosones de Goldstone aparecen como QNMs no masivos. Un hecho un poco menos bien sabido es que los bosones de Goldstone no tienen necesariamente relaciones de dispersión lineales, aún en teorías de campos relativistas. Dependiendo de si su relación de dispersión es proporcional a una potencia par o impar del momento son llamados de tipo I o de tipo II (ver [107] para más información). La aparición de bosones de Goldstone de tipo II también está relacionada a otro hecho, que el número de bosones de Goldstone no iguales al número de generadores rotos $[105,104,111]^{2}$. De hecho en el modelo holográfico la simetría $U(2)$ se rompe a $U(1)$ y consecuentemente hay tres generadores de simetría rotos pero sólo dos bosones de Goldstone holográficos fueron encontrados. Uno de ellos puede ser identificado con el modo de sonido usual con relación de dispersión

$$
\omega(k)=v_{s} k+(b-i \Gamma) k^{2},
$$

donde $v_{s}$ es la velocidad del sonido, $b$ una corrección cuadrática en momento y $\Gamma$ la constante de atenuación del sonido. Los bosones de Goldstone tipo II por otro lado tienen una relación de dispersión

$$
\omega(k)=(B-i C) k^{2},
$$

sin parte lineal. Todas las constantes que aparecen en estas expresiones son dependientes de la temperatura y satisfacen $v_{s}\left(T_{c}\right)=0, b\left(T_{c}\right)=B\left(T_{c}\right)$ y $C\left(T_{c}\right)=\Gamma\left(T_{c}\right)$.

Vamos a investigar la estabilidad del superfluido a través de un análisis de QNM del modelo $U(2)$. Esto va a dar de manera automática nueve y valiosa información sobre el superfluido holográfico $U(1)$ usual ya que un subsector de las fluctuaciones en el modelo $U(2)$ es isomorfo al $U(1)$.

En la sección 6.3 vamos a seguir a $[136,137]$ y reproducir el diagrama de fases basándonos en la comparación de las energías en las fases con superflujo y la fase normal. Entonces estudiaremos el espectro de modos QNM en presencia del superflujo. En particular vamos a estudiar la dependencia angular de la velocidad del sonido. Vamos a encontrar que a medida que la velocidad del superflujo se incrementa la velocidad del sonido en la dirección opuesta la superflujo disminuye y eventualmente desaparece a cierta velocidad crítica $v_{c}$. Al aumentar la velocidad del superfluido más aun esta velocidad del sonido esta velocidad del sonido se hace negativa y esto debe ser interpretado como la aparición de un estado de energía negativa en el espectro. En principio esto debería ser suficiente para argumentar una inestabilidad pero básicamente gratis el análisis de modos QNM puede darnos una señal aun más claro de esta inestabilidad. Es bien sabido que la parte imaginaria de los QNMs tiene que vivir en el semiplano inferior. Si ellos fallan y migran al semiplano superior un modo creciente con

\footnotetext{
${ }^{1}$ Un modelo holográfico similar fue también introducido en [159].

${ }^{2}$ Resultados recientes en bosones Goldstone tipo II pueden ser encontrados en [113, 165, 116, 166]. En un contexto holográfico modos de Goldstone tipo II fueron encontrados también en [106].
} 
amplitud $\phi \propto \exp (\Gamma t)$ aparece en el espectro. No es necesario que este modo tenga momento cero. De hecho veremos que si incrementamos la velocidad del superfluido más allá de su valor crítico la parte imaginaria de la frecuencia quasinormal del modo de sonido se muda al semiplano superior. Esto lo hace logrando un máximo para cierto momento finito. Veremos que este comportamiento es necesario en orden de conectar con el diagrama de fases de la fase normal. Luego, moviéndonos un poco a un costado, estudiamos las conductividades en la fase con superflujo. Esto fue hecho antes pero sólo en el sector transverso y acá se presentan resultados para el sector longitudinal.

Finalmente investigamos el destino de los bosones de Goldstone tipo dos en el modelo $U(2)$. Vamos a estudiar tanto el modelo gaugeado como no gaugeado de [89]. El criterio de Landau sugiere que en estos setups no sean capaces de sostener un superflujo finito ya que mín $\frac{\epsilon(p)}{p}=0$ para relaciones de dispersión cuadráticas. De nuevo podemos estudiar también la parte imaginaria. Vamos a encontrar una vez más polos en el semiplano superior para todas las temperaturas y velocidades del superfluido para los dos modelos ${ }^{3}$.

Vamos ahora a mencionar ciertos defectos de nuestro análisis. Trabajamos siempre en el límite de desacoplamiento en el cual las fluctuaciones de la métrica estás suprimidas. Entonces no vemos el patrón de primer y segundo (y cuarto) sonidos típicos para superfluidos. En el límite de desacoplo sólo el cuarto sonido, las fluctuaciones del condensado, sobreviven. Otro defecto es que aplicamos el criterio de Landau sólo a los QNMs. Como en el Helio superfluido es probable que existan otras excitaciones, como vórtices, que podrían modificar el valor de la velocidad crítica. La pregunta de cómo los solitones determinan la velocidad del superfluido crítica fue estudiada en [167].

Resulta interesante comparar nuestros resultados con la dependencia angular de la velocidad del sonido en un modelo a acoplamiento débil como el recientemente estudiado en [168]. Más recientemente, en [169] el diagrama de fases de un superfluido a acoplamiento débil fue estudiado, obteniéndose resultados cualitativamente muy similares a los nuestros.

\subsection{Repaso sobre la teoría de Landau-Ginzburg}

Para comenzar, repasemos rápidamente qué tienen Landau y Ginzburg que decirnos sobre superfluidos, siguiendo los pasos de [168].

Empecemos por el lagrangiano

$$
\mathcal{L}=\partial_{\mu} \varphi \partial^{\mu} \varphi^{*}-m^{2}|\varphi|^{2}-\lambda|\varphi|^{4}
$$

donde $\varphi$ es un campo escalar, $m \geq 0$ su masa y la constante de acoplamiento $\lambda>0$. El lagrangiano es invariante frente a rotaciones de $U(1) \varphi \rightarrow e^{i \alpha} \varphi$ lo que implica una carga conservada. Nótese que la condición de tener una masa real implica que el término cuadrático del potencial sea positivo y una rotura espontánea de simetría puede ocurrir sólo si introducimos un potencial químico asociado a la carga conservada. Si el potencial químico es más grande que la masa $m$ se forma un condensado de Bose-Einstein de partículas. En este formalismo, vamos a introducir dicho potencial químico a través de una dependencia temporal de la fase del parámetro de orden. Nótese que equivalentemente se podría hacer lo

\footnotetext{
${ }^{3}$ Modelos con un campo de gauge U(1) y dos complejos escalares similares a nuestro modelo no gaugeado fueron estudiados antes en [145] y más recientemente en [148] (ver también [147]). En esos casos los dos escalares tenían masas distintas y esto debería prevenir la aparición de modos de Goldstone tipo II.
} 
mismo introduciendo una fuente externa a través de un campo de gauge temporal constante, como hacemos en los modelos holográficos.

Vamos a elegir un ansatz para el condensado de Bose-Einstein de la forma

$$
\varphi(x)=\frac{e^{i \psi(x)}}{\sqrt{2}} \rho(x) .
$$

Acá, $\rho(x)$ es el módulo y $\psi(x)$ la fase del condensado. Insertando este ansatz obtenemos el lagrangiano a nivel árbol

$$
\mathcal{L}=\frac{1}{2} \partial_{\mu} \rho \partial^{\mu} \rho+\frac{\rho^{2}}{2}\left(\partial_{\mu} \psi \partial^{\mu} \psi-m^{2}\right)-\frac{\lambda}{4} \rho^{4} .
$$

$\mathrm{Al}$ ignorar las fluctuaciones vamos a poder hacer una traducción a las ecuaciones y magnitudes hidrodinámicas en el caso más simple allá [170].

Las ecuaciones clásicas de movimiento son

$$
\begin{aligned}
\square \rho & =\rho\left(\sigma^{2}-m^{2}-\lambda \rho^{2}\right), \\
\partial_{\mu}\left(\rho^{2} \partial^{\mu} \psi\right) & =0,
\end{aligned}
$$

donde hemos abreviado

$$
\sigma^{2} \equiv \partial_{\mu} \psi \partial^{\mu} \psi
$$

La expresión de teoría de campos para la corriente de carga conservada y el tensor de energíamomento son

$$
\begin{aligned}
j^{\mu} & =\frac{\partial \mathcal{L}}{\partial\left(\partial_{\mu} \psi\right)}=\rho^{2} \partial^{\mu} \psi \\
T^{\mu \nu} & =\frac{2}{\sqrt{-g}} \frac{\delta(\sqrt{-g} \mathcal{L})}{\delta g_{\mu \nu}}=2 \frac{\partial \mathcal{L}}{\partial g_{\mu \nu}}-g^{\mu \nu} \mathcal{L}=\partial^{\mu} \rho \partial^{\nu} \rho+\rho^{2} \partial^{\mu} \psi \partial^{\nu} \psi-g^{\mu \nu} \mathcal{L}
\end{aligned}
$$

Para el tensor de energía-momento usamos la definición gravitacional mediante la introducción de una métrica general $g$ la cual, después de tomar derivadas, fijamos de manera tal que sea aquella del espacio de Minkowski. Esta definición garantiza que $T^{\mu \nu}$ es simétrico y conservado. Como $j^{\mu}$ también es conservada, tenemos

$$
\partial_{\mu} j^{\mu}=0, \quad \partial_{\mu} T^{\mu \nu}=0,
$$

que son las ecuaciones hidrodinámicas. Como esperamos del teorema de Noether, la ecuación de conservación de la corriente y la ecuación para la fase $\psi$ coinciden.

Una solución a las ecuaciones de movimiento es

$$
\begin{aligned}
\psi & =p_{\mu} x^{\mu}, \\
\rho & =\sqrt{\frac{p^{2}-m^{2}}{\lambda}} .
\end{aligned}
$$

Este ansatz corresponde a un superfluido infinito fluyendo de manera uniforme. La densidad y el flujo están determinados por las componentes de $p_{\mu}$, que son simples números y no funciones de $x$ y que no están restringidos por las ecuaciones de movimiento. El valor de $p_{\mu}$ 
es determinado por las condiciones de borde, que especifican la topología de la configuración de campos, o sea el número de veces que la fase gira alrededor a medida que atravesamos la región espaciotemporal en la que reside el superfluido.

Metiendo esta solución en el lagrangiano tenemos

$$
\mathcal{L}=\frac{\left(\sigma^{2}-m^{2}\right)^{2}}{4 \lambda}
$$

El lagrangiano on-shell a nivel árbol nos da la presión.

Ahora exploramos la conexión entre las cantidades de teoría de campos y las hidrodinámicas. El enfoque hidrodinámico incluye escribir una corriente y un tensor de energía momento en términos de cantidades fluido-mecánicas

$$
\begin{aligned}
j^{\mu} & =n_{s} v^{\mu}, \\
T^{\mu \nu} & =\left(\epsilon_{s}+P_{s}\right) v^{\mu} v^{\nu}-g^{\mu \nu} P_{s},
\end{aligned}
$$

donde $v^{\mu}$ es la velocidad del superfluido y por definición $v_{\mu} v^{\mu}=1$. Para identificar $n_{s}, \epsilon_{s}$ y $P_{s}$ tenemos que ir al marco de referencia en el que el superfluido está en reposo $v^{\mu}=$ $(1,0,0,0)$, donde no hay flujo de carga, energía o momento: $j^{i}=T^{0 i}=0$. Entonces tenemos sencillamente que $j^{0}=n_{s}, T^{00}=\epsilon_{s}, T^{i j}=\delta^{i j} P_{s}$, de manera que $n_{s}, \epsilon_{s}$ y $P_{s}$ son la densidad de carga, densidad de energía y presión en el marco de referencia en el que el superfluido está en reposo. Estos pueden ser expresados de manera covariante usando las contracciones correspondientes

$$
n_{s}=\sqrt{j^{\mu} j_{\mu}}=v^{\mu} j_{\mu}, \quad \epsilon=v_{\mu} v_{\nu} T^{\mu \nu}, \quad P_{s}=-\frac{1}{3}\left(g_{\mu \nu}-v_{\mu} v_{\nu}\right) T^{\mu \nu} .
$$

Para relacionar estas cantidades hidrodinámicas con sus equivalente de campos podemos usar las ecuaciones (6.11) y (6.12)

$$
n_{s}=\sigma \frac{\sigma^{2}-m^{2}}{\lambda}, \quad v^{\mu}=\frac{\partial^{\mu} \psi}{\sigma}=\gamma\left(1, \mathbf{v}_{s}\right)
$$

donde $\mathbf{v}_{s}$ es la componente espacial de la velocidad del superfluido con un factor de Lorentz $\gamma=1 / \sqrt{1-\mathbf{v}_{s}^{2}}$.

Para $\epsilon_{s}$ y $P_{s}$ en cambio obtenemos

$$
P_{s}=\frac{\left(\sigma^{2}-m^{2}\right)^{2}}{4 \lambda}, \quad \epsilon_{s}=\frac{\left(3 \sigma^{2}+m^{2}\right)\left(\sigma^{2}-m^{2}\right)}{4 \lambda} .
$$

Nótese que $m$ es la única escala de masas de nuestro lagrangiano. La traza del tensor de energía-momento $T_{\mu}^{\mu}=\epsilon_{s}+\rho_{s}-3 P_{s}=m^{2} \rho^{2}$ se anula para $m=0$. Este caso sería considerar una CFT a potencial químico finito, lo cual se acerca aun más a nuestros estudios holográficos.

Finalmente la densidad de superfluido $\rho_{s}$ se define a través de una expansión para $\mathbf{v}_{s}$ chico y resulta en

$$
\rho_{s}=\epsilon_{s}+P_{s}=\sigma^{2} \frac{\sigma^{2}-m^{2}}{\lambda}
$$

Entonces, a temperatura nula se puede pegar perfectamente la teoría de Landau para un campo escalar con la descripción hidrodinámica de un fluido perfecto. Como es un fluido 
único, siempre se puede mover uno al marco de referencia en el que el fluido está quieto y eso mata mucha de la física interesante. Los autores de [168] mostraron que para el caso de la teoría de Landau a temperatura finita el ansatz de fluido único ya no es válido y el superfluido se convierte en un sistema de dos fluidos.

La manera más inmediata de añadir una segunda componente a la hidrodinámica de un solo fluido parece ser

$$
\begin{aligned}
j^{\mu} & =n_{n} u^{\mu}+n_{s} \frac{\partial^{\mu} \psi}{\sigma}, \\
T^{\mu \nu} & =\left(\epsilon_{n}+P_{n}\right) u^{\mu} u^{\nu}-g^{\mu \nu} P_{n}+\left(\epsilon_{s}+P_{s}\right) \frac{\partial^{\mu} \psi \partial_{\nu} \psi}{\sigma^{2}}-g^{\mu \nu} P_{s} .
\end{aligned}
$$

Acá sólo añadimos los términos correspondientes a un fluido normal a los ya definidos para un superfluido. En partícula, hemos introducido la velocidad del fluido normal $u^{\mu}$, con $u^{\mu} u_{\mu}=1$. Como consecuencia, podemos definir el marco de referencia de reposo para el fluido normal fijando $u^{\mu}=(1,0,0,0)$. Obviamente, si usamos este marco de referencia todavía vamos a tener una corriente encendida para el superfluido. En otras palabras, en contraste con el caso del superfluido a temperatura cero, no hay un marco de referencia para el cual la presión sea isotrópica. Para ver como pegar los coeficientes hidrodinámicos con aquellos provenientes de la teoría de campos, es necesario calcular la acción efectiva térmica a un loop. Dicha cuenta puede ser encontrada en [168].

En nuestros superfluidos holográficos siempre tendremos en mente la imagen de un fluido de dos componentes, una normal y una superfluida. El marco de referencia elegido será aquel en el que el fluido normal está en reposo. No podemos hacer boost de Lorentz para cambiar de marco de referencia ya que no estamos considerando la dinámica de la métrica.

\section{3. $\quad$ El superfluido $U(2)$ con superflujo}

Consideremos el lagrangiano de bulk de un doblete escalar en la representación fundamental gaugeado con una simetría $U(2)$ [89, 159]

$$
S=\int d^{4} x \sqrt{-g} \mathcal{L}=\int d^{4} x \sqrt{-g}\left(-\frac{1}{4} F^{\mu \nu c} F_{\mu \nu}^{c}-m^{2} \psi^{\dagger} \psi-\left(D^{\mu} \psi\right)^{\dagger} D_{\mu} \psi\right),
$$

donde

$$
\psi=\sqrt{2}\left(\begin{array}{c}
\lambda \\
\Psi
\end{array}\right), \quad A_{\mu}=A_{\mu}^{c} T_{c}, \quad D_{\mu}=\partial_{\mu}-i A_{\mu}
$$

donde hemos incluido un factor $\sqrt{2}$ en la definición del campo escalar para que las ecuaciones estén de acuerdo con aquellas de [136]. Siguiendo [27] elegimos que la masa de escalar sea $m^{2}=-2 / L^{2}$. Los generadores de $U(2)$ serán

$$
T_{0}=\frac{1}{2} \mathbb{I}, \quad T_{i}=\frac{1}{2} \sigma_{i} .
$$

Como vamos a trabajar en la aproximación de prueba no incluimos a la métrica entre los grados de libertad dinámicos sino que simplemente consideramos (6.25) en el background de 
una brana negra de Schwarzschild-AdS

$$
\begin{aligned}
d s^{2} & =-f(r) d t^{2}+\frac{d r^{2}}{f(r)}+\frac{r^{2}}{L^{2}}\left(d x^{2}+d y^{2}\right), \\
f(r) & =\frac{r^{2}}{L^{2}}-\frac{M}{r} .
\end{aligned}
$$

El horizonte se encuentra en $r_{H}=M^{1 / 3} L^{2 / 3}$ y su temperatura de Hawking es $T=3 r_{H} / 4 \pi L^{2}$. Mediante los reescaleos correspondientes podemos fijar $L=r_{H}=1$ y trabajar con coordenadas adimensionales.

Si queremos encontrar soluciones correspondientes a un condensado con velocidad de superfluido no nula debemos proceder como explicaremos. Primero nótese que el escalar $\lambda(r)$ puede ser fijado a cero mediante una trasformación de gauge de $U(2)$. Para el escalar $\Psi$ pedimos que el comportamiento dominante en el borde se anule. Mediante una transformación de gauge $U(1)$ podemos pedir que $\Psi$ sea real.

Ahora necesitamos definir que queremos decir con velocidad de superfluido. Discutamos esto desde la perspectiva de una teoría de campos. En un superfluido multi-componente con simetría $U(2)$ podemos en principio definir cuatro supercorrientes

$$
J_{a}^{\mu}=\Phi^{\dagger} T_{a} \nabla^{\mu} \Phi-\left(\nabla^{\mu} \Phi\right)^{\dagger} T_{a} \Phi
$$

donde $\nabla^{\mu}=\partial^{\mu}-i A_{a}^{\mu} T_{a}$ es la derivada covariante y $\Phi$ es la función de onda del condensado que transforma como un doblete frente a $U(2)$. Su el condensado es tal que alguna de las componentes espaciales de las corrientes no se anula podemos hablar de un estado con superflujo no nulo. Mediante una transformación de gauge podemos siempre asumir que el condensado va a tomar la forma estándar, e.g. $\Phi=(0, \phi)^{T}$ y representar el superflujo no nulo en términos de campos de gauge externos. Como estamos interesados en romper la simetría $U(2)$ de manera espontánea a $U(1)$ vamos solamente a permitir un campo de gauge no nulo $U(1)$ correspondiente al generador $T_{0}$. Más aun mediante una rotación de $S O(2)$ podemos hacer que el campo de gauge apunte en la dirección $x$. De (6.29) es fácil ver que semejante superflujo implica corrientes no nulas $J_{x}^{(0)}$ y $J_{x}^{(3)}$. Para encontrar soluciones con una carga no trivial también tenemos que buscar soluciones con un potencial químico no trivial. Una vez más, con el fin de preservar la simetría $U(2)$ vamos a permitir un potencial químico sólo para la carga overall $U(1)$.

Volviendo ahora a holografía, estas consideraciones determinan que el ansatz para los campos de gauge sea de la forma

$$
A^{(0)}=A_{t}^{(0)}(r) d t+A_{x}^{(0)}(r) d x, \quad A^{(3)}=A_{t}^{(3)}(r) d t+A_{x}^{(3)}(r) d x .
$$

Si bien introducimos fuentes sólo para $A^{(0)}$ el hecho de que también la corriente $J_{\mu}^{(3)}$ sea no nula exige que $A^{(3)} \neq 0$. La interpretación física de este hecho es que el sistema fuerza la aparición de una densidad de carga $\rho^{(3)} \neq 0$ (como fue explicado ya en [89]) y una corriente $J_{x}^{(3)}$ en el vacío con superflujo. Esto está de hecho relacionado íntimamente con la presencia de bosones de Goldstone tipo en el espectro [109].

A esta altura es importante notar que la identificación de más arriba es sólo válida en la fase superfluida, esto es, cuando $\Psi \neq 0$. Un valor de background constante para el campo de gauge $A_{x}$ en la fase normal no tiene sentido físico ya que no hay noción de superflujo. 
Por las razones señaladas más arriba, elegimos las condiciones de borde asintóticas para los campos de gauge

$$
\begin{array}{llrl}
A_{t}^{(0)}(r \rightarrow \infty) & =2 \bar{\mu}, & & A_{t}^{(3)}(r \rightarrow \infty)=0, \\
A_{x}^{(0)}(r \rightarrow \infty) & =2 \bar{S}_{x}, & & A_{x}^{(3)}(r \rightarrow \infty)=0,
\end{array}
$$

donde $\bar{\mu}$ debe ser identificado con el potencial químico de la teoría dual y $\bar{S}_{x}$ está relacionada con la velocidad del superflujo. Hemos incluido un factor dos en la definiciones de $\bar{\mu}$ y $\bar{S}_{x}$ por el siguiente motivo. Las ecuaciones de background pueden ser reescritas como aquellas del modelo $U(1)$ de $[136,137]$ utilizando las redefiniciones de los campos

$$
\begin{aligned}
A_{0} & =\frac{1}{2}\left(A_{t}^{(0)}-A_{t}^{(3)}\right), & \xi & =\frac{1}{2}\left(A_{t}^{(0)}+A_{t}^{(3)}\right), \\
A_{x} & =\frac{1}{2}\left(A_{x}^{(0)}-A_{x}^{(3)}\right), & \varsigma & =\frac{1}{2}\left(A_{x}^{(0)}+A_{x}^{(3)}\right),
\end{aligned}
$$

para los cuales las ecuaciones de background quedan

$$
\begin{aligned}
\Psi^{\prime \prime}+\left(\frac{f^{\prime}}{f}+\frac{2}{r}\right) \Psi^{\prime}+\left(\frac{A_{0}^{2}}{f^{2}}-\frac{A_{x}^{2}}{r^{2} f}-\frac{m^{2}}{f}\right) \Psi & =0, \\
A_{0}^{\prime \prime}+\frac{2}{r} A_{0}^{\prime}-\frac{2 \Psi^{2}}{f} A_{0} & =0, \\
A_{x}^{\prime \prime}+\frac{f^{\prime}}{f} A_{x}^{\prime}-A_{x} \frac{2 \Psi^{2}}{f} & =0, \\
\xi^{\prime \prime}+\frac{2}{r} \xi^{\prime} & =0, \\
\varsigma^{\prime \prime}+\frac{f^{\prime}}{f} \varsigma^{\prime} & =0 .
\end{aligned}
$$

Se puede corroborar que recuperamos el sistema $U(1)$ que describe el superconductor holográfico $U(1)$ en presencia de velocidad para el superfluido (véase por ejemplo [164]). El potencial químico $\bar{\mu}$ es por lo tanto el potencial químico correspondiente al campo $A_{0}$ que actúa como la componente temporal del (único) campo de gauge, y $A_{x}$ actúa como la componente espacial del único campo de gauge [136, 137, 164]. Esto muestra de manera explícita que el background del modelo $U(2)$ es idéntico a aquel del superconductor $U(1)$, aun a velocidad del superfluido no nula.

Una consecuencia inmediata del hecho que las ecuaciones de fondo son aquellas del superfluido holográfico $U(1)$ es que, a primera vista, el sistema $U(2)$ parece capaz de acomodar un superflujo. Sin embargo, como ya fue argumentado, esto va en directa contradicción con el criterio de Landau para superfluidez [163] debido a la presencia de un bosón de Goldstone tipo II en el espectro. Por supuesto, el haber encontrado soluciones a las ecuaciones de movimiento no dice nada aun sobre la estabilidad. De hecho, como veremos explícitamente, los Goldstone de tipo II van a convertirse en un modo inestable haciendo que la toda la solución en el caso $U(2)$ sea inestable.

Las ecuaciones (6.33)-(6.35) son no lineales y deben ser resueltas usando métodos numéricos. Nótese que (6.36) y (6.37) están desacopladas. Corresponden a la simetría $U(1)$ preservada luego de la rotura $U(2) \rightarrow U(1)$. El comportamiento asintótico de los campos 
cerca del borde conforme es

$$
\begin{aligned}
& A_{0}=\bar{\mu}-\frac{\bar{\rho}}{r}+\ldots, \\
& A_{x}=\bar{S}_{x}-\frac{\overline{\mathcal{J}}_{x}}{r}+\ldots, \\
& \Psi=\frac{\psi_{1}}{r}+\frac{\psi_{2}}{r^{2}}+\ldots
\end{aligned}
$$

Las cantidades asintóticas están relacionadas con las físicas mediante

$$
\begin{array}{ll}
\bar{\mu}=\frac{3}{4 \pi T} \mu, & \bar{\rho}=\frac{9}{16 \pi^{2} T^{2}} \rho, \\
\bar{S}_{x}=\frac{3}{4 \pi T} S_{x}, & \overline{\mathcal{J}}_{x}=\frac{9}{16 \pi^{2} T^{2}} \mathcal{J}_{x}, \\
\psi_{1}=\frac{3}{4 \pi T}\left\langle\mathcal{O}_{1}\right\rangle, & \psi_{2}=\frac{9}{16 \pi^{2} T^{2}}\left\langle\mathcal{O}_{2}\right\rangle .
\end{array}
$$

Estamos trabajando en el ensamble gran canónico, por lo que fijamos el potencial químico $\mu$. La temperatura viene definida por $T / \mu \propto 1 / \bar{\mu}$. Para estudiar la evolución del condensado como función de la velocidad del superfluido, la manera natural de proceder es trabajando con $S_{x} / \mu$ como nuestro parámetro libre además de la temperatura. Nótese que ambos modos asintóticos del campo escalar son de hecho normalizables [117]. De ahora en adelante nos vamos a quedar con la teoría $\mathcal{O}_{2}$ para la cual $\psi_{1}=0$ y $\left\langle\mathcal{O}_{2}\right\rangle$ es el vev del operador escalar de dimensión de masa dos en la teoría de campos dual. Nótese que los campos duales $\xi$ y $\zeta$ correspondientes al $U(1)$ no roto vienen dados por

$$
\begin{aligned}
& \xi=\bar{\mu}-\bar{\rho} / r, \\
& \zeta=\bar{S}_{x},
\end{aligned}
$$

aun cuando el condensado es no nulo.

Los valores del condensado como función de la temperatura y la velocidad del superfluido se muestran en la figura 6.1 que reproduce los resultados previos de [136, 137]. En el gráfico y en lo que queda del capítulo la temperatura es medida con respecto a la temperatura crítica de la transición de fases sin velocidad del superfluido, i.e. $T_{c} \approx 0,0587 \mu$.

\subsubsection{Energía libre}

En esta sección vamos a calcular la energía libre de la fase condensada y compararla con la energía libre de la fase no rota como fue hecho en [136, 137]. Después de la renormalización correspondiente de la acción on-shell euclídea y de usar las condiciones de contorno (6.38), la energía libre es

$$
F=-T S_{r e n}=-\bar{\mu} \bar{\rho}+\bar{S}_{x} \overline{\mathcal{J}}_{x}+\int_{1}^{\infty} d r\left(\frac{2 r^{2} A_{0}^{2}}{f}-2 A_{x}^{2}\right) \Psi^{2} .
$$

En la fase normal $\Psi=0$, regularidad en el horizonte fuerza a el campo de gauge $A_{x}$ a tener un perfil trivial en la dirección radial en el bulk y por lo tanto a no contribuir a la energía libre, i.e. $\overline{\mathcal{J}}_{x}=0$. Esto va en concordancia con el hecho de que en ausencia de campo escalar 


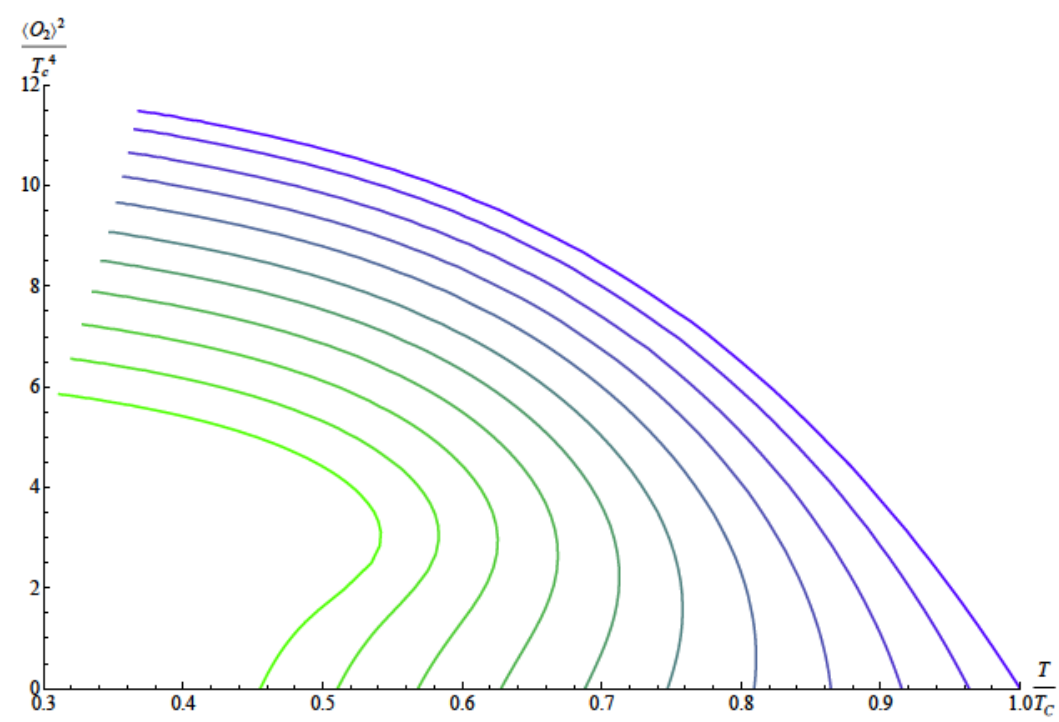

Figura 6.1: El condensado para diferentes valores de la velocidad del superfluido, yendo desde $\frac{S_{x}}{\mu}=0,005$ (derecha) hasta $\frac{S_{x}}{\mu}=0,530$ (izquierda).

no es posible prender una velocidad de superfluido. Prender la componente espacial de un campo de gauge ne la fase normal describe una transformación puramente de gauge que no afecta a la energía libre del sistema. En la fase rota en cambio, diferentes velocidades del superfluido son distinguibles físicamente. Es importante enfatizar que uno está comparando la fase normal (sin velocidad para el superfluido) con la fase superconductora para diferentes velocidades del superfluido y que la fase normal es inestable frente a la condensación sin superflujo para cualquier $T<T_{c}$. Por lo tanto, la relevancia física de esta comparación no resulta del todo clara. Vamos a ver más tarde que de hecho el criterio de Landau establece una temperatura de transición diferente para la fase con superfluido. Sin embargo la energía libre da un primer acercamiento natural al diagrama de fases del sistema.

En la figura 6.2 la energía libre de las fases normal y condensada es graficada para diferentes valores de $\frac{S x}{\mu}$. La diferencia en comportamiento para valores grandes y pequeños de la velocidad del superfluido es evidente. Para valores grandes de la velocidad del superfluido la transición de fases es de primer orden como puede ser observado en el panel izquierdo de la figura 6.2, indicada por un círculo vacío. Viniendo desde temperaturas bajas el sistema todavía puede ser recalentado hacia un estado metaestable hasta el punto de descomposición espinodal donde la susceptibilidad del parámetro de orden $\partial\langle\mathcal{O}\rangle / \partial \mu$ diverge, indicado por el punto relleno.

Para velocidades de superfluido bajar la energía libre de la fase normal y la de la fase con condensado se pegan de manera continua en una transición de fases de segundo orden. El espacio de fases correspondiente aparece en la figura 6.6 y reproduce el análisis previo de $[136,137]$.

La transición de fases obtenida de considerar tan solo la energía libre es sin embargo un engaño. Vamos a llamar a la temperatura para la cual la energía libre de la fase con condensado y superflujo y la energía libre de la fase normal coinciden $\tilde{T}$ de ahora en adelante. A aquella temperatura a la cual la transición de fases (de segundo orden) phase sin superfluido la llamaremos $T_{c}$. Como mostraremos ahora el superflujo se hace inestable a temperaturas 

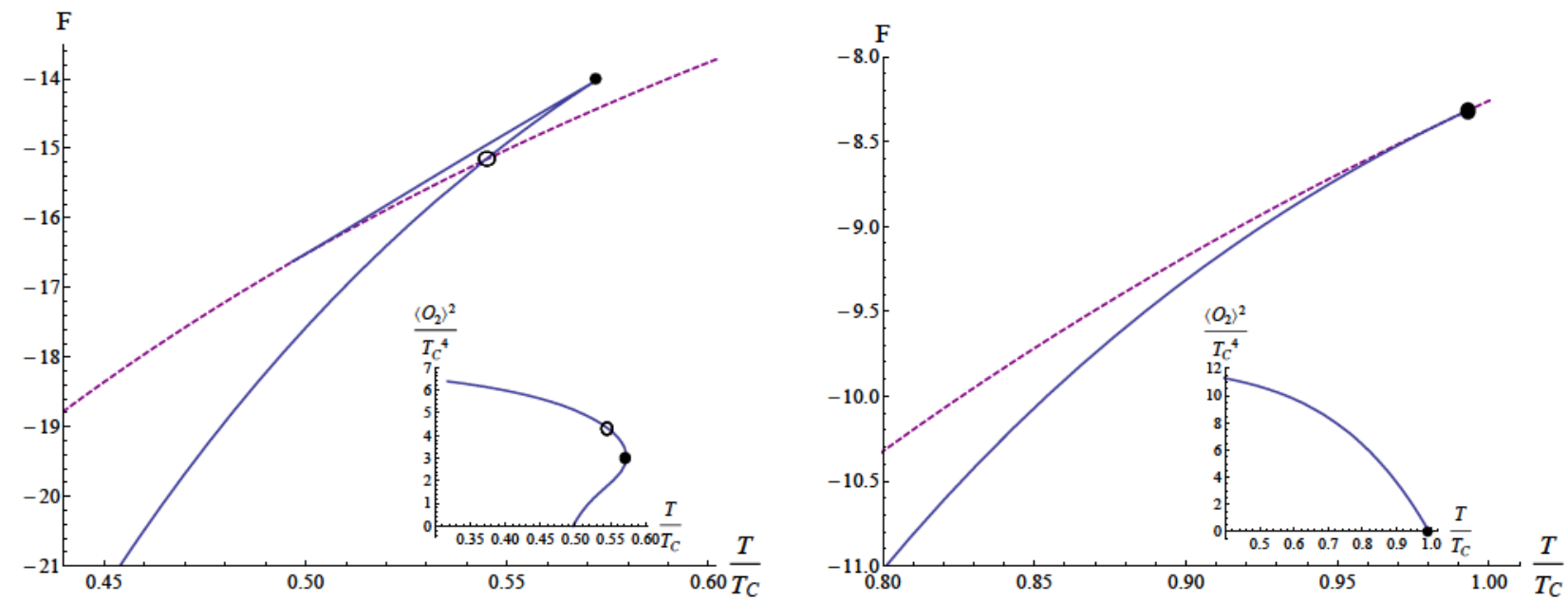

Figura 6.2: Energía libre para las fases condensada (línea continua) y normal (línea entrecortada) para $\frac{S_{x}}{\mu}=0,5$ (izquierda) y $\frac{S_{x}}{\mu}=0,05$ (derecha). Los plots pequeños muestran el comportamiento del condensado. El círculo vacío corresponde a la temperatura crítica $\tilde{T}$ mientras que el círculo relleno corresponde la punto espinodal (máximo recalentamiento).

por debajo de la de $\tilde{T}$ según dice el criterio de Landau aplicado a los modos de sonido. A la temperatura para la cual el superfluido vuelve a ser estable la llamaremos $T^{*}$.

\subsection{Criterio de Landau para el sector $U(1)$}

En esta sección analizamos el espectro de QNM del sector (0) - (3), que es idéntico al superconductor holográfico $U(1)$ original en presencia de superfluido [136, 137]. Nos concentramos en los QNM más bajos, los bosones de Goldstone tipo I, con especial énfasis en la velocidad y la constante de atenuación y su dependencia con la velocidad de superfluido y en el ángulo de propagación con respecto a la dirección del flujo.

Para estudiar el espectro de QNM consideramos perturbaciones linealizadas alrededor de los campos de background de la forma $\delta \phi_{I}=\delta \phi_{I}(r) \exp [-i(\omega t-|k| x \cos (\gamma)-|k| y \sin (\gamma)]$. Específicamente consideramos las fluctuaciones

$$
\begin{aligned}
\delta \hat{\Psi}^{T} & =(\eta(r), \sigma(r)), \\
\delta A^{(0)} & =a_{t}^{(0)}(r) d t+a_{x}^{(0)}(r) d x+a_{y}^{(0)}(r) d y, \\
\delta A^{(3)} & =a_{t}^{(3)}(r) d t+a_{x}^{(3)}(r) d x+a_{y}^{(3)}(r) d y,
\end{aligned}
$$

donde en el caso de las fluctuaciones de gauge vamos a trabajar con las combinaciones lineales ya definidas en $(6.32)$, i.e. $a_{\mu}^{(-)} \equiv \frac{1}{2}\left(a_{\mu}^{(0)}-a_{\mu}^{(3)}\right)$ y $a_{\mu}^{(+)} \equiv \frac{1}{2}\left(a_{\mu}^{(0)}+a_{\mu}^{(3)}\right)$. Las ecuaciones linealizadas son jodias y las escribimos en el apéndice B.1. Las técnicas utilizadas para resolver el sistema de ecuaciones son bien conocidas. No vamos aç elaborar en las mismas y referiremos al lector interesado a [100], [119] o al apéndice .

En las figuras 6.3 y 6.4 representamos la velocidad del sonido y la constante de atenuación para un bosón de Goldstone tipo I. Su relación de dispersión viene dada por (6.3) y a momento pequeño, con la excepción de que ahora la velocidad del sonido $v_{s} \mathrm{y}$ la constante 

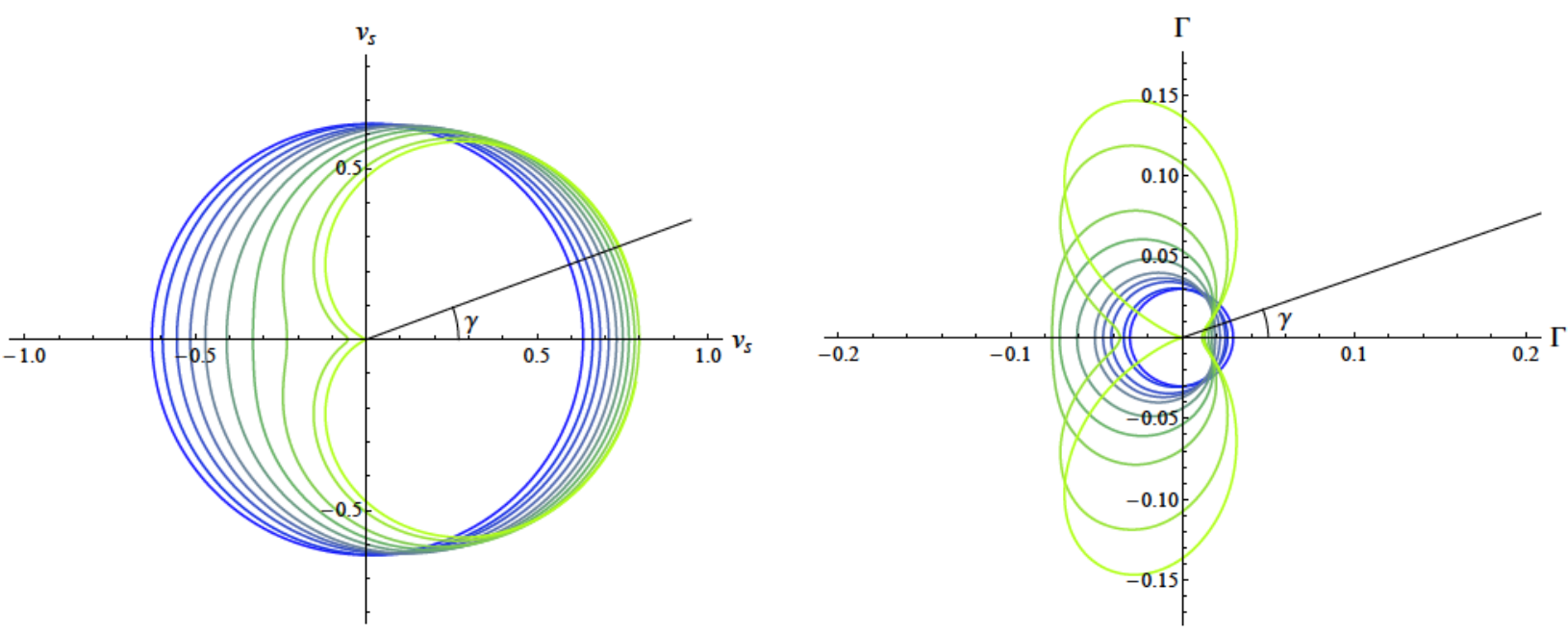

Figura 6.3: Velocidad del sonido y amortiguación para $T=0,7 T_{c}$ y varias velocidades de superfluido yendo de $S_{x} / \mu=0$ (azul) a $S_{x} / \mu=0,325$ (verde). El radio representa el valor absoluto de la velocidad del sonido (izquierda) y la constante de atenuación (derecha) como función del ángulo $\gamma$ entre el momento y la velocidad del superfluido.
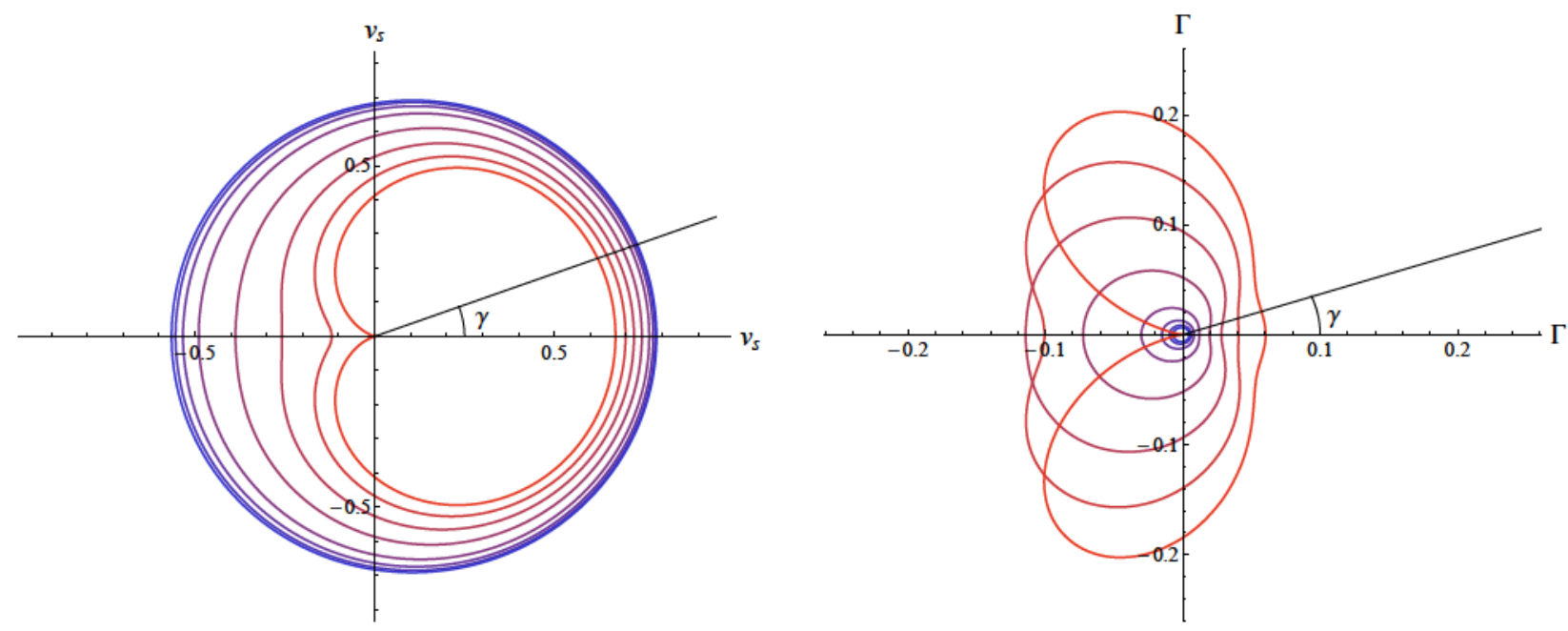

Figura 6.4: Velocidad del sonido (izquierda) y constante de atenuación (derecha) para $S_{x} / \mu=$ 0,2 como función del ángulo $\gamma \mathrm{y}$ para un rango de temperaturas yendo desde $T=0,85 T_{c}$ (rojo) hasta $T=0,57 T_{c}$ (azul).

de atenuación $\Gamma$ dependen del ángulo $\gamma^{4}$. La figura 6.3 muestra la dependencia angular de la velocidad del sonido y la constante de decaimiento para una temperatura fija y varios valores de la velocidad del superfluido. La figura 6.4 muestra lo mismo a velocidad del superfluido fija y para varios valores de la temperatura. Como uno esperaría para $S_{x} / \mu$ chico y temperaturas lo suficientemente bajas la velocidad del sonido y la constante de decaimiento son básicamente isotrópicas. A medida que la velocidad del superfluido es aumentada, o la temperatura es aumentada, los gráficos se hacen más y más asimétricos. La anisotropía del

\footnotetext{
${ }^{4}$ La constante real $b$ no juega ningún papel para momento lo suficientemente pequeño ya que la parte lineal proporcional a $v_{s}$ domina.
} 
sistema es tal que vemos un aumento de la velocidad del sonido y una disminución de la constante de decaimiento en dirección del superfluido.
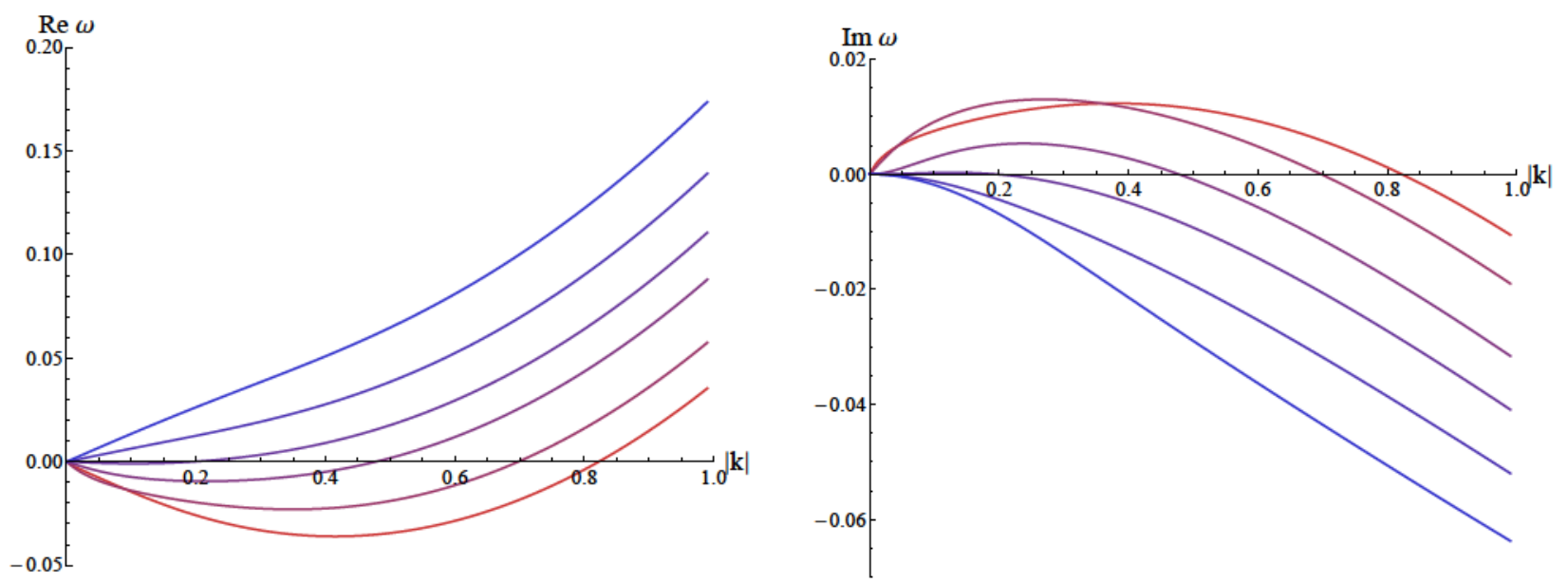

Figura 6.5: Partes real (izquierda) e imaginaria (derecha) de la frecuencia de los modos hidrodinámicos más bajos (modos de Goldstone tipo I) versus el momento a $S_{x} / \mu=0,1 \mathrm{y}$ $\gamma=\pi$ para diferentes temperaturas yendo desde $T=\tilde{T}=0,970 T_{c}$ (rojo) hasta $T=0,905 T c$ (azul). La inestabilidad aparece a $T^{*}=0,935 T_{c}$.

Las propiedades más interesantes fueron encontradas sin embargo en la dirección opuesta a la velocidad de superfluido . Como uno puede ver en ambos gráficos, a $\gamma=\pi$ la reducción en la velocidad del sonido es mayor y eventualmente tanto la atenuación como la velocidad del sonido se van a anular simultáneamente. Es importante enfatizar que esto ocurre por debajo de la temperatura $\tilde{T}$. Si uno continua incrementando la temperatura (o equivalentemente incrementando la velocidad del superfluido a temperatura fija) uno encuentra que las partes real e imaginaria de la temperatura cruzan al semiplano superior, como se muestra en la figura 6.5. Esto señala la aparición de un modo taquiónico. $T^{*}$ es la temperatura en que esta inestabilidad aparece y además la velocidad del sonido se hace negativa. Esta temperatura señala de hecho el fin de la fase superfluida de acuerdo con el criterio y por lo tanto la interpretamos como la temperatura de transición física.

En la figura 6.6 (izquierda) presentamos el diagrama de fases que emerge del análisis de QNMs. Para ilustrar la situación, en el gráfico de la derecha mostramos la relación de dispersión de los QNMs relevantes ${ }^{5}$ en tres puntos diferentes del diagrama de fases (los puntos fueron etiquetados con 1, 2, 3 en el gráfico de la izquierda). A $\tilde{T}<T<T_{c}$ en la fase normal (línea $3_{N}$ ), el modo que era responsable a de la transición a la fase superfluida homogénea phase sin velocidad del superfluido es corrido y se hace inestable a momento finito. Este comportamiento refleja el hecho de que el superfluido es inestable para $T \leq T_{c}$, siendo que el modo fue corrido en momento por el valor constante del campo $A_{x}$. A $T=\tilde{T}$ (líneas $2_{N, S}$ ) el modo más bajo se hace inestable a $k=0$. Es en este punto que la energía libre del superfluido iguala a aquella del fluido normal. Por lo tanto, el análisis de la energía libre, que sólo captura la dinámica a $k=0$, predice una transición de fase a esta temperatura. Para la velocidad de superfluido elegida en el gráfico la transición de fases es de segundo orden.

\footnotetext{
${ }^{5}$ En la fase no rota esto es simplemente el modo escalar más bajo, mientras que en la fase rota es el modo de sonido a $S_{x} / \mu$ fija.
} 


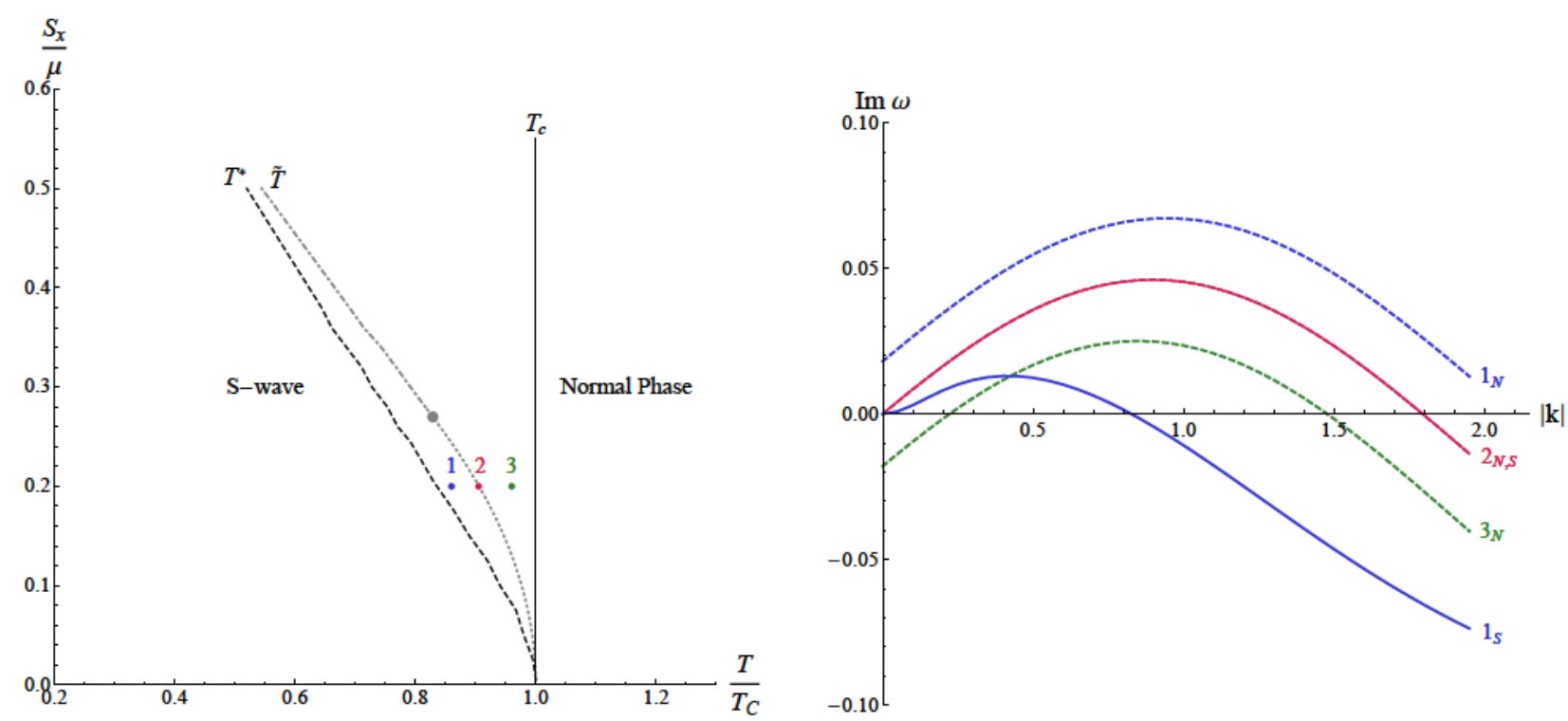

Figura 6.6: (Izquierda) Diagrama de fases posterior al estudio de los QNMs. La línea gris entrecortada corresponde a $\tilde{T}$, la temperatura de supuesta transición encontrada mediante análisis directo de la energía libre. En cierto punto (disco) la transición en la energía libre cambia de segundo (punteada) a primer orden (punteada-entrecortada). La línea negra sólida corresponde a la temperatura crítica en ausencia de velocidad del superfluido. La línea entrecortada negra señala la transición de fase física a $T^{*}$, temperatura para la cual la inestabilidad aparece. Los puntos 1, 2 y 3 indican los valores de la temperatura utilizados para hacer los gráficos de la derecha. (Derecha) Partes real e imaginaria de los QNMs más bajos para diferentes temperaturas fijando $S_{x} / \mu=0,2$ y $\gamma=\pi$. Las líneas entrecortadas fueron obtenidas en el background de la fase normal mientras que las líneas sólidas fueron calculadas en la fase superfluida.

Finalmente, el destino de los QNM para $T^{*}<T<\tilde{T}$ se muestra en la línea $1_{N}$ (para la fase normal) y $1_{S}$ (para la fase con superflujo homogéneo). Uno puede ver que el modo de Goldstone en la fase superfluida es inestable para un rango finito de momento. Sólo a $T^{*}$ este modo se hace inestable nuevamente como se muestra en la figura 6.5. Es a esta temperatura que la fase con superflujo homogénea se hace estable de acuerdo con el criterio de Landau ya que la velocidad del sonido se hace positiva (más aun la parte imaginaria de la relación de dispersión del QNM yace completamente en el semiplano inferior).

Por lo tanto los resultados de QNM indican que la transición de fases ocurre a una temperatura más baja $T^{*}<\tilde{T}$. De manera similar, si imaginamos al sistema a temperatura fija y empezamos a subir la velocidad del superfluido, tanto $v_{s}$ como $\Gamma$ se harán cero para algún valor de $S_{x} / \mu$, que podremos decir que es de hecho la temperatura crítica $v_{c}$ del superfluido, en el sentido del criterio de Landau.

Un hecho interesante es que la parte imaginaria del modo que exhibe la inestabilidad tiene un máximo a momento finito también. El hecho de que la inestabilidad aparezca a momento finito sugiere que podría haber una fase nueva y (meta)estable por encima de $T^{*}$ con un condensado con modulación espacial. Ejemplos de dichas inestabilidad hacia fase moduladas fueron estudiadas con anterioridad en [171, 172, 173, 174].

Recuérdese que el criterio de Landau está formulado únicamente en términos de $\operatorname{Re}(\omega)$. 
La velocidad crítica corresponde a la velocidad para la cual $v_{s}=0$, o equivalentemente al valor de $S_{x} / \mu$ para el cual $\operatorname{Re}(\omega)$ se hace negativa (ver la figura 6.5 ). El hecho que el criterio se enunciado para $\operatorname{Re}(\omega)$ refleja le hecho de que se mantenga aun a temperatura cero. A temperatura finita la relación de dispersión del modo no masivo se ve alterada debido a la velocidad del superfluido y la temperatura [163, 168], implicando que genéricamente el valor critico de $S_{x} / \mu$ a temperatura fija no corresponde a la velocidad del sonido a dicha temperatura y velocidad del superfluido nula.

Un comentario extra con respecto a la fase en la que se encuentra el sistema para $T_{c}>T>\tilde{T}$. El hecho de que en la fase no rota el QNM más bajo sea inestable en este régimen (ver la línea $3_{N}$ en la figura 6.6) por supuesto indica que la fase normal es inestable. Comentemos un poco sobre esto. Como el condensado se anula en la fase normal, no existe ninguna noción física de velocidad en esta fase; diferentes elecciones de $A_{x}$ son simplemente diferentes elecciones de sistema de referencia. En particular, un $A_{x}$ constante actúa simplemente corriendo el momento, como puede ser visto del hecho que el máximo del QNM está centrado a un momento igual al valor del campo de gauge en el borde conforme. Por lo tanto, la fase normal es inestable para temperaturas más bajas que la crítica $T_{c}$ hacia la formación de superfluido sin superflujo. Por otro lado, conocemos que la solución con condensado homogéneo y velocidad finita no existe en esta región y peor aun es inestable para $T>T^{*}$. Vemos dos posibilidades para completar el diagrama de fases en esta región. Primero, el sistema podría simplemente caer en el estado de vacío, que es el condensado sin superflujo. A $S_{x} / \mu$ finita esto es todavía una solución que minimiza la energía aun que con un condensado que no es real pero tiene una dependencia espacial en la fase tal que $\vec{\nabla} \Phi=0$. Este es simplemente el estado homogéneo sin superflujo transformado de gauge. Por otro lado, el hecho de que hayamos encontrado una inestabilidad a momento finito en el rango de temperaturas $T^{*}<T<\tilde{T}$ podría indicar que hay una fase (metaestable) modulada espacialmente aun en el rango $T^{*}<T<T_{c}$, es decir un superfluido con bandas. Debido a la aparición suave del modo inestable a $T^{*}$ esperamos que la transición de fases sea de segundo orden, aunque esto debería ser estudiado en detalle mediante la construcción del background inhomogéneo correcto. Dicha construcción está en proceso, pero por lo pronto diremos que es heavy metal.

Antes de cambiar de tema, resulta reconfortante comparar nuestros resultados con aquellos obtenidos recientemente para el modelo $\lambda \varphi^{4}$ presentado en 6.2. Resultados tomado de las referencias [168] y [169] son presentados en la figura 6.7. Esto corresponde a un análisis en el límite de acoplamiento débil del modelo de Landau, pero de todas maneras si esperamos que nuestros agujeros negros tengan algunas propiedades en común, si confiamos ciegamente en la conjetura. En el gráfico de la izquierda se muestra la velocidad del primer y segundo sonido del modelo para distintas velocidades y temperaturas. Se ve que el primer sonido tiene un comportamiento cualitativo muy parecido al encontrado en nuestra figura 6.3. El gráfico de la derecha es un diagrama de fases, teniendo en cuenta el criterio de Landau. El mismo muestra una gran similitud con nuestra figura 6.6.

\subsubsection{Conductividades longitudinales en el sector $U(1)$}

En esta sección vamos a computar las conductividades del sector (0) - (3) en presencia de velocidad para el superfluido. Por lo que tenemos entendido, sólo las conductividades transversas fueron computadas hasta el momento (ver por ejemplo [164, 138]). Nosotros por 

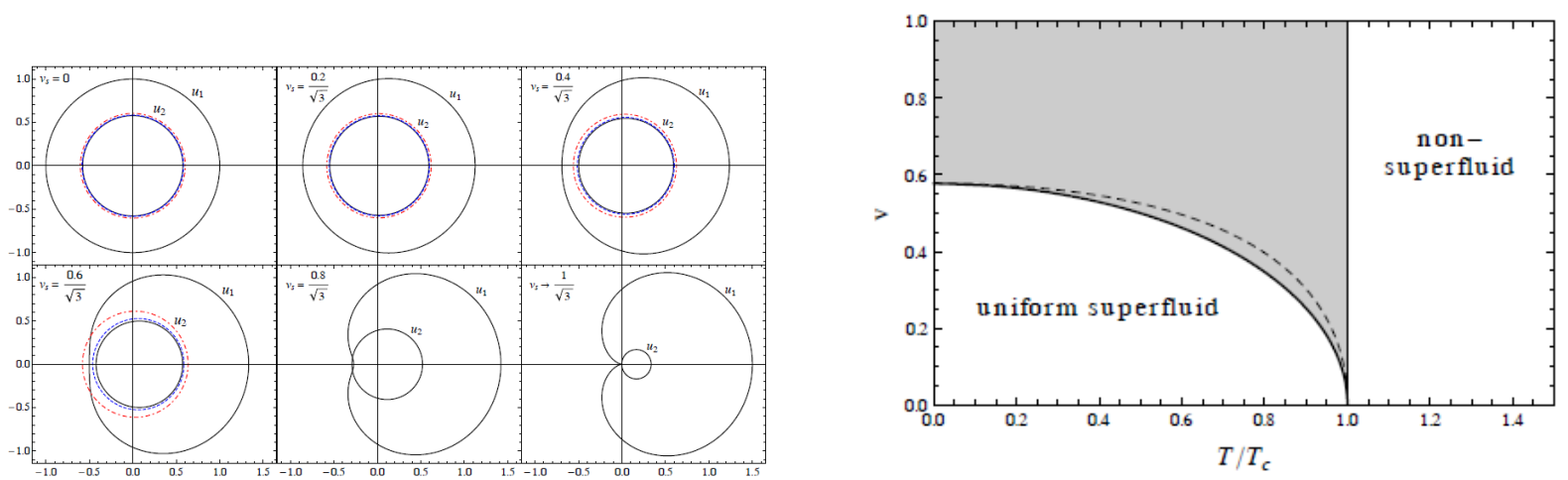

Figura 6.7: (Izquierda, [168]) Velocidades del primer y segundo sonido $u_{1}, u_{2}$ para distintas velocidades $\left|\mathbf{v}_{s}\right|$ entre 0 y $1 / \sqrt{3}$. Todas las velocidades son medidas en el sistema de referencia en el que el fluido normal está en reposo. Se usaron las temperaturas $T=0$ (negro), $T / \mu=$ 0,02 (azul) y $T / \mu=0,04$ (rojo). (Derecha, [169]) Diagrama de fases del modelo. Dentro de un ansatz homogéneo no hay una fase estable en la región sombreada. Nótese que la inestabilidad a $T=0$ ocurre justo a $\left|\mathbf{v}_{s}\right|=1 / \sqrt{3}$, donde la velocidad del sonido se hace negativa según puede verse en el gráfico de la izquierda.

el contrario, nos vamos a concentrar en la conductividades longitudinales. Las mismas son calculadas mediante la fórmula de Kubo

$$
\sigma=\frac{i}{\omega}\left\langle J^{x} J^{x}\right\rangle
$$

de la función de dos puntos

$$
\mathcal{G}_{I J}=\lim _{\Lambda \rightarrow \infty}\left(\mathcal{A}_{I M} \mathcal{F}_{k J}^{M}(\Lambda)^{\prime}\right),
$$

donde la matriz $\mathcal{A}$ puede ser leída de la acción on-shell. $\mathcal{F}$ es el propagador matricial bulk-toboundary normalizado para se la matriz unidad en el borde. Como estamos sólo interesados en las entradas de la matriz correspondientes a $\left\langle J^{x} J^{x}\right\rangle$ y la matriz $\mathcal{A}$ es diagonal, sólo tenemos que leer un elemento, i.e. $\mathcal{A}_{x x}=-\frac{f(r)}{2}$. Con la intención de construir el propagador bulk-toboundary uno necesita un conjunto completo de soluciones linealmente independientes para las perturbaciones del escalar y del campo de gauge. Esto implica resolver las ecuaciones dadas en el apéndice B.1 a momento cero. El método sigue de cerca a aquel detallado en [119]. Nótese que hay un acoplamiento sobreviviente entre el sector escalar y los campos de gauge mediado por $A_{x}$. Esto hace que el cálculo de conductividades sea más complicado que en el caso sin superflujo.

Nuestros resultados muestran una pequeña desviación con respecto a lo encontrado para velocidad de superfluido cero. La característica mas interesante es un pico a frecuencias pequeñas que aparece debido al acoplamiento del campo de gauge y los escalares inducido por la velocidad del superfluido. En las figuras 6.8 y 6.9 presentamos los resultados para diferentes valores de $S_{x} / \mu$. Como es de esperarse, cuando la velocidad del superfluido es pequeña y estamos lejos de la temperatura crítica la dependencia en la frecuencia es la misma que la encontrada en [27]. Cerca de $T^{*}$ un salto aparece para $\omega \approx 0$. Esto indica la presencia de un modo con un gap imaginario pequeño. El modo responsable de este comportamiento es precisamente el modo pseudo difusivo descripto en [100]. Debido a la 

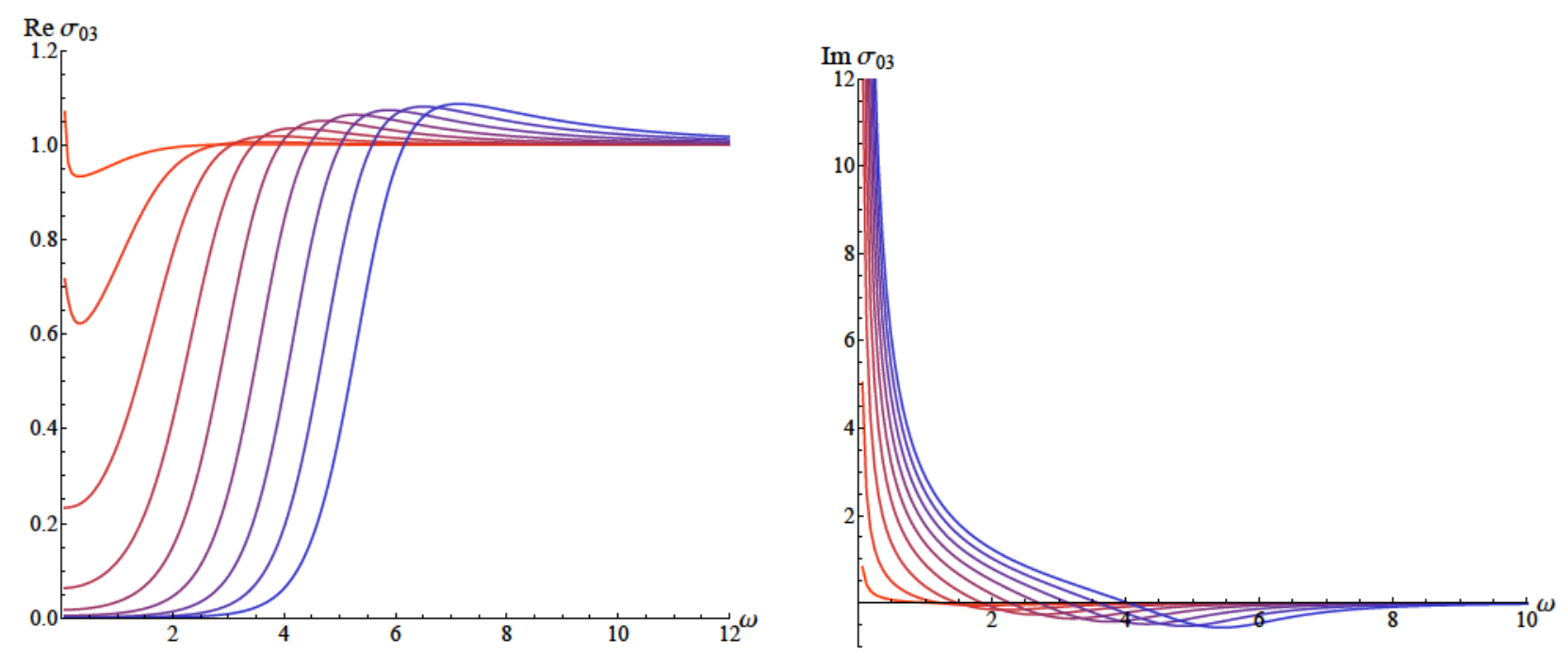

Figura 6.8: Gráficos para la parte real (izquierda) e imaginaria (derecha) de la conductividad para $S_{x} / \mu=0,05$ fija. Diferentes líneas corresponden a diferentes temperaturas yendo desde $T=0,99 T_{c}$ (rojo) a $T=0,38 T_{c}$ (azul).

simetría conservada $U(1)$ de la fase no rota, existe un modo difusivo (de masa cero) en el espectro de QNM de la teoría. Una vez que la simetría es espontáneamente rota, este modo desarrolla un gap puramente imaginario que crece a medida que incrementamos la temperatura. Por lo tanto, para temperaturas lo suficientemente altas por debajo de la crítica, el gap del modo pseudodifusivo a $k=0$ es muy pequeño y esto implica la aparición de un pico en la conductividad como podemos ver en las figuras. Si bajamos la temperatura, el salto empieza a desaparecer simplemente porque el gap del modo pseudodifusivo se hace más grande. Aunque este modo ya fue presentado en el análisis de conductividades sin superflujo, es sólo en nuestro caso que afecta la conductividad, ya que el acoplamiento a momento cero entre el sector escalar y el de campos de gauge ocurre mediante $A_{x}$. El tamaño del pico es proporcional al tamaño del acoplamiento, i.e. crece con $S_{x} / \mu$.

\subsection{Criterio de Landau para bosones de Goldstone tipo II}

En la sección anterior estudiamos el espectro de QNM más bajos contenidos en el sector (0) - (3) o $U(1)$ de la teoría para varios valores de la velocidad de superfluido y un ángulo arbitrario entre el momento y la dirección del superflujo. En esta sección vamos a extender el análisis al sector (1) - (2), que es particular del modelo $U(2)$ de [89] y contiene modos de Goldstone tipo II en el espectro, cuya relación de dispersión viene dada por (6.4) en el límite hidrodinámico.

Las ecuaciones que describen el sistema pueden encontrarse en el apéndice B.2. En este caso hemos elegido siempre al momento en la dirección opuesta al superfluido, porque como veremos este modo será siempre inestable. Junto con las perturbaciones escalares descritas 

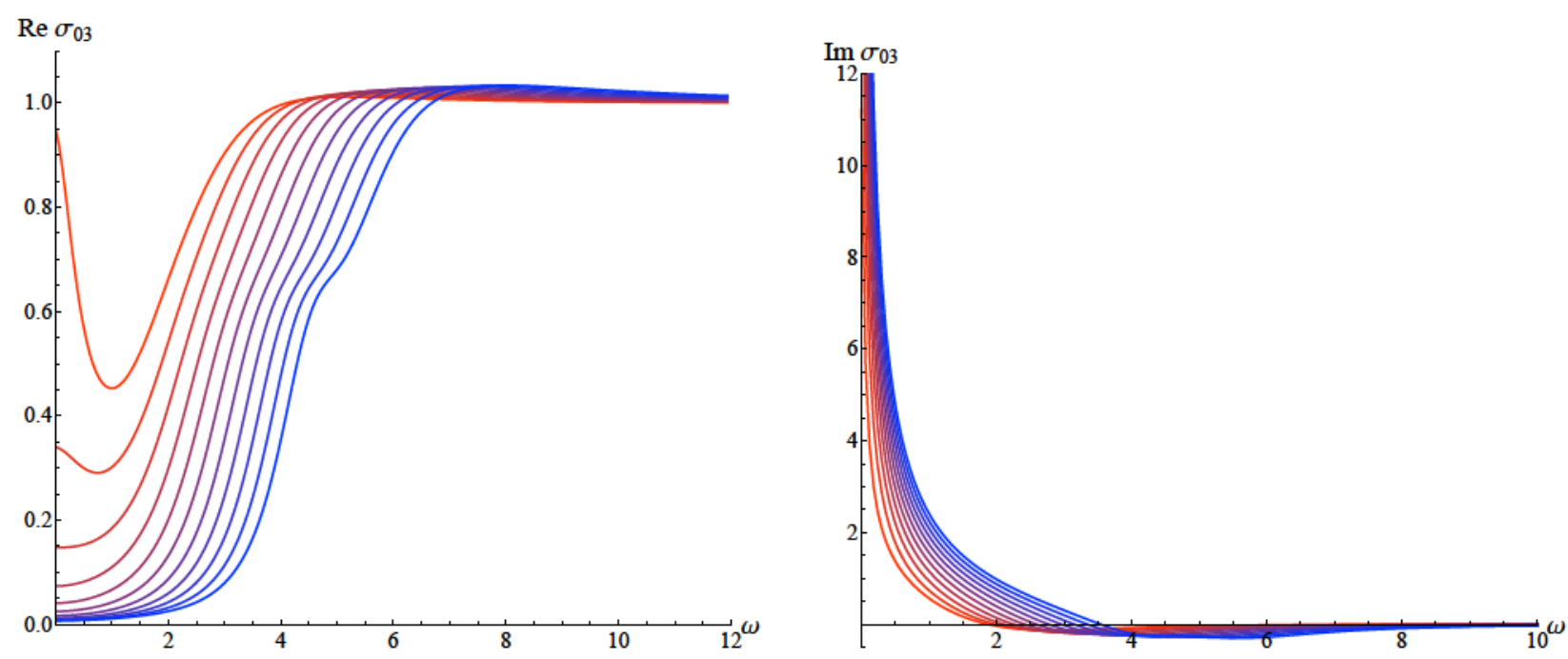

Figura 6.9: Gráficos para la parte real (izquierda) e imaginaria (derecha) de la conductividad para $S_{x} / \mu=0,4$ fija. Diferentes líneas corresponden a diferentes temperaturas en el rango $T=0,35 T_{c}(\operatorname{azul})-0,65 T_{c}($ rojo $)$.

por (6.42) tenemos que considerar las siguientes perturbaciones de gauge en el sector (1)-(2)

$$
\begin{aligned}
& A^{(1)}=a_{t}^{(1)}(t, r, x) d t+a_{x}^{(1)}(t, r, x) d x, \\
& A^{(2)}=a_{t}^{(2)}(t, r, x) d t+a_{x}^{(2)}(t, r, x) d x .
\end{aligned}
$$

Una vez más usamos el método del determinante de [119] para encontrar los QNMs en este sector. Nuestros resultados aparecen resumidos en la figura 6.10, donde la relación de dispersión de los QNM más bajos se muestra a una determinada velocidad de superfluido. Hemos corroborado que este resultado es cualitativamente el mismo para varios valores de $S_{x} / \mu$.

Los modos de Goldstone tipo II se hacen inestables para velocidades de superfluido arbitrariamente pequeñas y temperaturas por debajo de $\tilde{T}$. Sin embargo, una diferencia importante aparece con respecto al sector $U(1)$. El modo taquiónico no se hace estable a ninguna temperatura por debajo de $\tilde{T}$, a diferencia con el sector $(0)-(3)$, no hay un análogo de la temperatura $T^{*}$ en este sector. Este comportamiento puede ser fácilmente interpretado como un reflejo del criterio de Landau para la superfluidez en nuestro setup holográfico: de acuerdo con (6.2), la velocidad crítica debe ser nula para sistemas en los que se pueden encontrar modos de Goldstone tipo II, por lo tanto para cualquier $T<\tilde{T}$ la fase superfluida no es estable a velocidad del superfluido finita. Además nótese que el pico ocurre a momentos más altos a medida que bajamos la temperatura. De hecho, como uno puede ver de la figura 6.10 , al bajar la temperatura por debajo de $\tilde{T}$ el máximo en $\operatorname{Im}(\omega)$ primero se incrementa pero luego empieza a bajar a medida que la temperatura disminuye. Al mismo tiempo, se mueve para valores más grandes del momento.

Nótese que gráficos análogos a los de las figuras 6.3 y 6.4 no tienen sentido en el modelo $U(2)$, ya que el sector $(1)-(2)$ es inestable a toda temperatura a la que fuimos capaces de corroborar. 

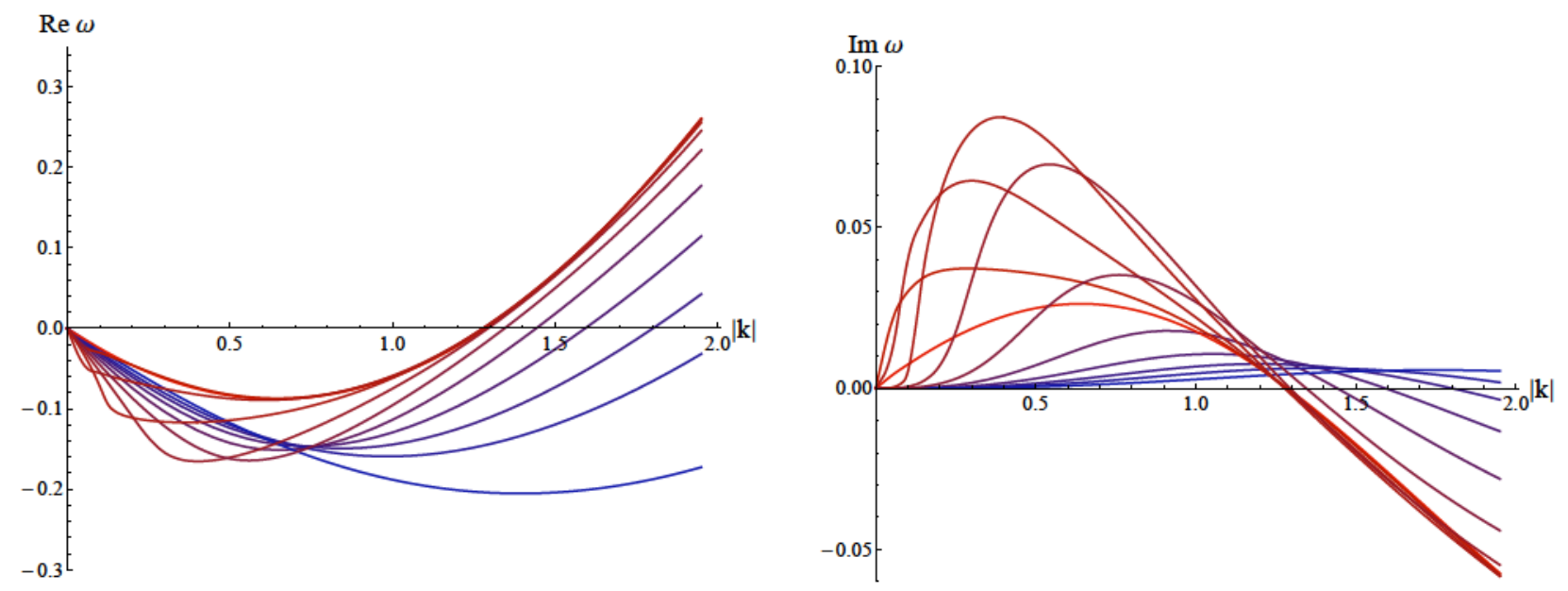

Figura 6.10: Partes real (izquierda) e imaginaria (derecha) de la relación de dispersión de los QNM más bajos del sector (1) - (2) en el modelo gaugeado fijando $S_{x} / \mu=0,15$ y para un rango de temperaturas yendo desde $T=\tilde{T}=0,95 T_{c}$ (rojo) hasta $T=0,45 T_{c}$ (azul) y momento en la dirección antiparalela al superflujo.

\subsection{1. $\quad$ El modelo Bivio}

En el capítulo 4, definimos un modelo no gaugeado en el cual no hay campos de gauge de $S U(2)$ dinámicos en el bulk. Este modelo tenue una simetría global $S U(2)$ y una simetría local $U(1)$ en el bulk. La teoría de campos dual no tiene por lo tanto los generadores de la simetría $S U(2)$ en su espectro de operadores. Sin embargo, como hemos visto un modo de Goldstone tipo II, quizás inesperado, aparece en el espectro de QNM del modelo.

El modelo Bivio viene dado básicamente por la misma acción (6.25) si nos quedamos tan sólo con el campo de gauge $U(1)$ overall. De hecho esto corresponde al simple modelo $U(1)$ con dos campos escalares con la masa degenerada y por lo tanto una simetría global $S U(2)$ accidental.

La solución de background es de nuevo aquella del superfluido $U(1)$, por lo tanto la solución con superflujo puede se acomodada también en el modelo no gaugeado. La diferencia es que los modos de Goldstone tipo II aparecen ahora en las fluctuaciones de la componente superior del campo escalar $\eta$, cual ecuación de avenimiento es

$$
f \eta^{\prime \prime}+\left(f^{\prime}+\frac{2 f}{r}\right) \eta^{\prime}+\left(\frac{\left(\omega+A_{0}\right)^{2}}{f}-\frac{\left(k-A_{x}\right)^{2}}{r^{2}}-m^{2}\right) \eta=0
$$

y está completamente desacoplada de las fluctuaciones de los demás campos. Como fue notado en [89] el cambio en el background debido a la aparición del condensado es suficiente para disparar los modos de Goldstone tipo II.

Resulta remarcable que los modos de Goldstone tipo II sea todavía inestable a cualquier temperatura por debajo de $\tilde{T}$ para cualquier valor finito de velocidad del superfluido. Nótese que al no incluir las cargas conservadas para la simetría $S U(2)$, el modelo no satisface todos los teoremas con respecto a la existencia de modos de Goldstone [89]. Sin embargo, el criterio de Landau para estabilidad es todavía válido.

El modelo no gaugeado presenta una característica distintiva con respecto al modelo gaugeado. El valor del momento en el máximo ahora decrece a medida que bajamos la 
temperatura. Esto se muestra en la figura 6.11, donde la relación de dispersión del modo de Goldstone tipo II a velocidad de superfluido fija y para un buen rango de temperaturas es graficado. Para valores arbitrarios de la velocidad del superfluido se obtienen resultados cualitativamente similares.
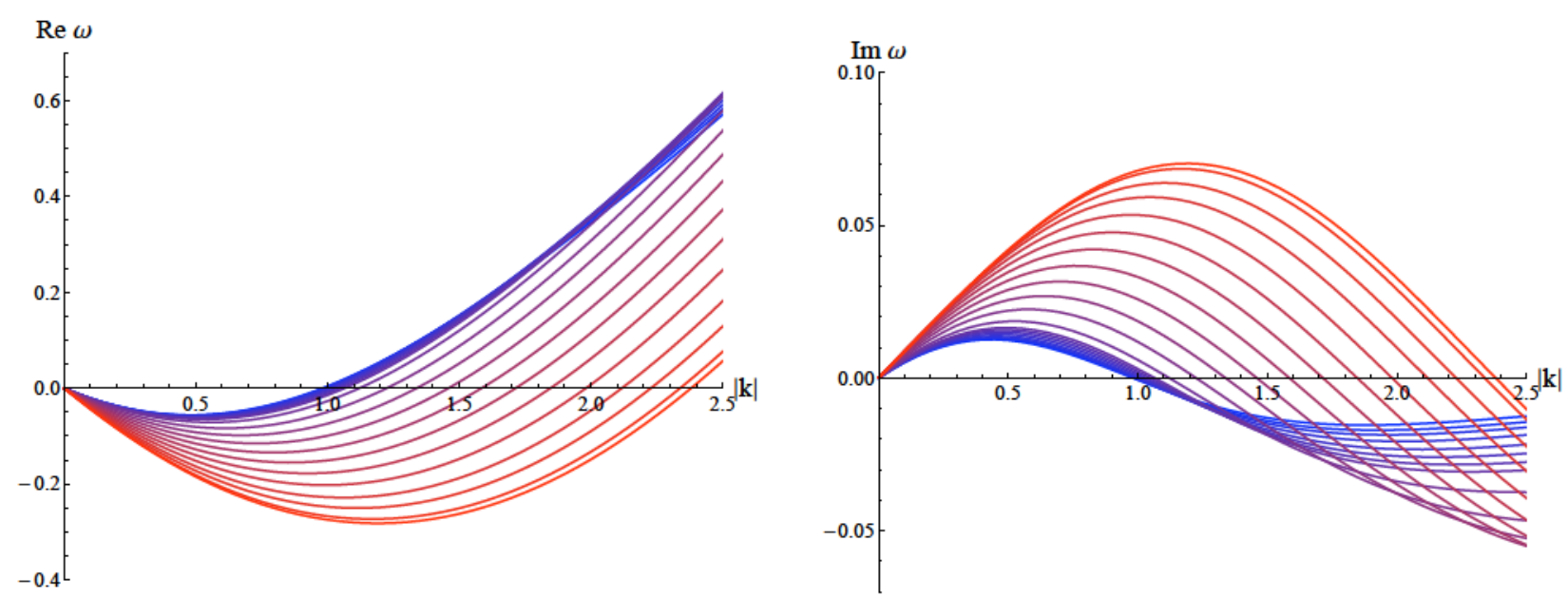

Figura 6.11: Partes real (izquierda) e imaginarias (derecha) de la relación de dispersión de los QNM más bajos en el sector (1) - (2) del modelo Bivio fijando $S_{x} / \mu=0,25$ y para un rango de temperaturas yendo desde $T=\tilde{T}=0,853 T_{c}$ (rojo) hasta $T=0,306 T_{c}$ (azul). El momento considerado es antiparalelo a la dirección del superfluido.

\subsection{Conclusiones}

Hemos estudiado la realización holográfica del criterio de Landau para superfluidez. El estudio fue motivado por la aparición de modos de Goldstone de tipo II en el modelo (6.25). La naturaleza cuadrática de la relación de dispersión de los modos de Goldstone de tipo II debería ser responsable de llevar al sistema fuera de la fase superfluida para velocidades de superfluido arbitrariamente pequeñas.

Sacando ventaja del hecho que el superconductor s-wave holográfico $U(1)$ está contenido en (6.25), vamos a profundizar el criterio de Landau para bosones de Goldstone tipo I. Si intentamos responder sobre la estabilidad de la fase condensada a velocidad de superfluido finita el análisis de la energía libre no nos da la respuesta correcta. El espectro de QNM contiene un modo taquiónico a momento finito para temperaturas $T^{*}<T<\tilde{T}$. Según su definición, $\tilde{T}$ es la temperatura a la cual la energía libre de la fase con condensado coincide con la energía libre de la fase normal. En contraste, $T^{*}$ es la temperatura a la cual la inestabilidad taquiónica aparece. Entonces, el superfluido homogéneo es estable sólo para $T<T^{*}$, como se muestra en la figura 6.6. Los resultados para la velocidad del sonido como función del ángulo $\gamma$ entre la dirección de propagación y la dirección de velocidad del superfluido, resumido en las figuras 6.3 y 6.4 , son perfectamente consistentes con los resultados de estabilidad: a $T=T^{*}$ y $\gamma=\pi$ la velocidad del sonido se hace cero. Esta condición puede considerarse equivalente al criterio de Landau y señala la existencia de una velocidad crítica por encima de la cual el superfluido deja de ser estable. 
Como el máximo de la parte imaginaria del modo inestable tiene un tiene un número de onda no nulo resulta natural sugerir que habrá otra fase, espacialmente modulada, para $T>$ $T^{*}$. La naturaleza de esta nueva fase es sin embargo desconocida y dejamos su construcción explicara para futuras investigaciones .

También hemos calculado la conductividad longitudinal para varias velocidades del superfluido. Hasta donde sabemos, no fueron calculadas anteriormente. Vemos un pico a $\omega=0$, debido al acoplamiento con la componente espacial del campo de gauge $A_{x}$. El pico disminuye a medida que bajamos la temperatura hasta quedar completamente suprimido (ver la figura 6.8). Creemos que este aumento de la conductividad DC es causado por el gap del modo pseudodifusivo $[100,89]$ que en presencia de velocidad del superfluido comienza a tener un papel protagónico debido al acoplamiento entre los sectores de gauge y escalares que ocurre aun para $k=0$.

Moviéndonos para el sector (1) - (2), resolvimos el impacto del superflujo en los bosones de Goldstone tipo II. Encontramos que el criterio de Landau es efectivo para velocidades arbitrariamente pequeñas como se muestra en la figura 6.10. El taquión persiste en todo el rango de temperaturas y para cualquier valor (finito) de velocidades de superfluido que fuimos capaces de estudiar. Por lo tanto, que la velocidad del superfluido crítica para este sector se anula, en completo acuerdo con el criterio de Landau para modos con relación de dispersión $\omega \propto k^{2}$. Un resultado análogo vale para los modos de Goldstone tipo II en el modelo Bivio. 


\title{
Capítulo 7
}

\section{Palabras finales}

\author{
"Then he waited, marshalling his thoughts and brooding over his still untested \\ powers. For though he was master of the world, he was not quite sure what to do \\ next.
}

But he would think of something."

Arthur C. Clarke

La aplicación de métodos holográficos a fenómenos de materia condensada está recién en pañales. En esta tesis se mostraron algunos de los últimos avances en este campo, todos ellos en el contexto de superfluidez holográfica

En el capítulo 2 se resumió el trabajo original [27] para luego considerar cómo el ruido altera algunas de las propiedades del sistema. En el capítulo 3 se trabajó en cambio con el modelo p-wave [26], obteniendo soluciones con backreaction, lo que nos permitió estudiar la entropía de entrelazamiento.

Luego, se combinaron los contenidos de materia de los superconsuctores tipo s y p, para dar origen a un superconductor $U(2)$ cuyas características se estudiaron durante el resto de esta tesis. Este modelo resultó contener varias características interesantes, como la realización de bosones de Goldstone tipo II, conductividades muy similares a la del grafeno y transiciones de fases entre distintos órdenes superconductores. Por último también estudiamos la capacidad de estos superfluidos para acomodar un superflujo.

Hasta ahora, los resultados vienen mostrando una satisfactoria similitud con lo esperado para sistemas físicos, lo cual es reconfortante e incrementa el entusiasmo y optimismo en esta rama paralela del estudio de sistemas de materia condensada. Sin embargo, hay que reconocer que las limitaciones y obstáculos que aun quedan por sortear son grandes.

Para comenzar, teorías gravitatorias débilmente acopladas hasta donde se sabe son duales a teorías de campos en el límite de $N$ grande. Este tipo de sistemas por lo pronto parecen alejados de aquellos que se estudian en materia condensada. Otra limitación parece ser que los métodos holográficos sólo tratan con descripciones efectivas de bajas energías del sistema real. Siendo este el caso, no parece posible obtener detalles de la física microscópica subyacente, a escalas en las que la red pasa a ser importante.

Sin embargo, la esperanza es que las teorías con duales holográficos tengan características exóticas universales, provenientes del acoplamiento fuerte. De ser este el caso, la holografía podría dar ejemplos sencillos donde los cálculos sean seguibles sobre estas características universales, siendo de utilidad para una mejor comprensión del problema subyacente. Otro 
aspecto prometedor es que la holografía sería capaz de proveer ejemplos sencillos de sistemas sin descripción de quasipartículas.

En fin, teorías con duales gravitatorios son teorías exóticas bien definidas contra las cuales los conceptos de materia condensada pueden evaluarse. Esto debe ser hecho sin ninguno de los recursos propios de teorías débilmente acopladas: uno puede responder preguntas sobre cargas, corrientes y parámetros de orden pero no sobre electrones o fonones. Con un poco de suerte, además puedan ser capaces de arrojar un poco de luz sobre la física de superconductores no convencionales.

\section{L.I.F.E.G.O.E.S.O.N.}

Durante la escritura y reescritura de esta tesis, algunas de las promesas proyectadas para el futuro comenzaron a realizarse. Para el lector interesado esta tesis continua en las referencias [175, 176, 177]. 


\section{Apéndice A}

\section{Sobre bosones de Goldstone tipo II}

\section{A.1. Relaciones de Kramers-Kronig matriciales}

La función espectral matricial genérica viene definida por

$$
\rho_{i j}(x)=\left\langle\left[\mathcal{O}_{i}(x), \mathcal{O}_{j}(0)\right]\right\rangle,
$$

donde $\mathcal{O}_{i}$ son operadores hermíticos. Su comportamiento bajo conjugación hermítica es

$$
\rho(x)^{\dagger}=\rho(-x)=-\rho(x)^{t} .
$$

Correspondientemente, la transformada de Fourier $\tilde{\rho}(k)=\int d^{4} x e^{-i k x} \rho(x)$ también satisface el conjunto de identidades

$$
\tilde{\rho}(k)^{\dagger}=\tilde{\rho}(k)=-\tilde{\rho}(-k)^{t} .
$$

En particular esto significa que los componentes de la diagonal son reales y antisimétricos frente a $k \rightarrow-k$. Uno puede estar también interesado en el comportamiento frente a $\omega \rightarrow-\omega$. Tomemos ahora $k=(\omega, \mathbf{q})$. Para teorías con invariancia rotacional la función espectral puede depender sólo de $\mathbf{q}^{2}$. Por lo tanto las componentes de la diagonal serán reales e impares en $\omega$

$$
\rho_{i i}\left(\omega, \mathbf{q}^{2}\right)=\rho_{i i}\left(\omega, \mathbf{q}^{2}\right)^{*}=-\rho_{i i}\left(-\omega, \mathbf{q}^{2}\right) .
$$

Para las componentes fuera de la diagonal sin embargo, sólo si uno impone simetría de paridad o de reversión temporal puede uno probar que los términos fuera de la diagonal deben ser funciones pares o impares de la frecuencia. En nuestro caso reversión temporal viene rota por la presencia de un potencial químico. Mayores restricciones pueden sin embargo ser obtenidas si se supone que la teoría es invariante frente a $\mathbf{x} \rightarrow-\mathbf{x}$. Para un número impar de dimensiones espaciales podríamos usar los operadores de paridad $P$ para tomar $\mathbf{x} \rightarrow-\mathbf{x}$. En el caso de dos dimensiones espaciales que estudiamos podemos tomar a $P$ como una rotación en $\pi$ (para un número arbitrario de dimensiones pares $D=2 n$ podríamos tomar el ángulo $\pi$ para todas las rotaciones en el plano $i, i+1$-ésimo para todo $i \leq n)$. Este operados $P$ actúa como $P \mathcal{O}_{i}(t, \mathbf{x}) P^{-1}=\sigma_{i} \mathcal{O}_{i}(t,-\mathbf{x})$ con $\sigma_{i}= \pm 1$. En dimensión espacial impar $\sigma_{i}$ es la paridad del operador. En dimensión espacial par $\sigma_{i}=-1$ si $\mathcal{O}_{i}$ es al componente de un vector espacial. Por lo tanto

$$
P\left[\rho_{i j}(t, \mathbf{x})\right]=\sigma_{i} \sigma_{j} \rho_{i j}(t,-\mathbf{x}) .
$$


invariancia frente a $P$ implica $\rho_{i j}(t, \mathbf{x})=\sigma_{i} \sigma_{j} \rho_{i j}(t,-\mathbf{x})$, lo cual implica para las transformadas de Fourier que

$$
\tilde{\rho}_{i j}(\omega, \mathbf{q})=-\sigma_{i} \sigma_{j} \tilde{\rho}_{i j}(-\omega, \mathbf{q})^{*} .
$$

De manera tal que las entradas fuera de al diagonal son funciones pares o impares de $\omega$ dependiendo en los signos de $\sigma_{i}$. En el caso en que los operadores transforman de la misma manera frente al operador paridad esto significa que la parte real (imaginaria) de las componentes fuera de la diagonal es una función impar (par) de la frecuencia.

Desde la función espectral, como definida en (A.1) podemos definir dos propagadores causales, las funciones de Green avanzadas y retardadas

$$
\begin{aligned}
& G_{R}(x)=-i \Theta(t) \rho(x), \\
& G_{A}(x)=i \Theta(-t) \rho(x),
\end{aligned}
$$

donde $x=(t, \mathbf{x})$. Usando (A.3), uno puede probar la siguiente relación entre las transformadas de Fourier de estos

$$
\tilde{G}_{R}(k)=\tilde{G}_{R}(-k)^{*}=\tilde{G}_{A}(k)^{\dagger} .
$$

De acá, vemos que la parte real (imaginaria), $\operatorname{Re}\left(G_{R}\right)\left(\operatorname{Im}\left(G_{R}\right)\right)$, es par (impar) bajo $k \rightarrow-k$. Podemos calcular la transformada de Fourier of de la función de Green retardada, la cual viene dada por la convolución de la transformadas e Fourier de la función escalón Heaviside $\tilde{\Theta}(\omega)$ con la transformada de Fourier de la función espectral $\tilde{\rho}(k)$,

$$
\tilde{G}_{R}(\omega, \mathbf{q})=-i \int_{-\infty}^{\infty} \tilde{\Theta}(\omega-\mu) \tilde{\rho}(\mu, \mathbf{q}) \frac{d \mu}{2 \pi} .
$$

Usando la transformada de Fourier de la función escalón

$$
\tilde{\Theta}(\omega)=\frac{i}{\omega+i \epsilon}
$$

y el teorema de Sokhatsky-Weierstrass obtenemos

$$
\tilde{G}_{R}(\omega, \mathbf{q})=\mathcal{P} \int_{-\infty}^{\infty} \frac{\tilde{\rho}\left(\omega^{\prime}, \mathbf{q}\right)}{\omega-\omega^{\prime}} \frac{d \omega^{\prime}}{2 \pi}-\frac{i}{2} \tilde{\rho}(\omega, \mathbf{q})
$$

donde $\mathcal{P}$ denota el valor principal. De la hermiticidad de $\tilde{\rho}(k)$ vemos que podemos considerar a (A.11) como la separación de $\tilde{G}^{R}(k)$ en sus partes hermíticas y antihermíticas, y encontrar que la función espectral puede ser calculada de la parte anti-hermítica de la transformada de Fourier de la función de Green retardada

$$
\tilde{\rho}(k)=i\left[\tilde{G}_{R}(k)-\tilde{G}_{R}(k)^{\dagger}\right] \equiv 2 i \tilde{G}_{R}^{(A)}(k),
$$

donde la $(A)$ significa antihermítico ${ }^{1}$. Metiendo este resultado en (A.11) y tomando la parte hermítica $(H)$ a ambos lados llegamos a que

$$
\tilde{G}_{R}^{(H)}(\omega)=\frac{i}{\pi} \mathcal{P} \int_{-\infty}^{\infty} \frac{G_{R}^{(A)}\left(\omega^{\prime}\right)}{\omega-\omega^{\prime}} d \omega^{\prime},
$$

\footnotetext{
${ }^{1}$ Usando (A.9) siempre podemos trabajar con las funciones de Green retardadas $G_{R}$.
} 
que no es nada más que al relación de Kramers-Krönig para funciones de Green matriciales. Ésta es complementada por la relación conjugada que intercambia las partes hermíticas y antihermíticas. Imponiendo invariancia frente a $P$ y usando (A.6) y (A.11) se sigue que la función de Green satisface

$$
\tilde{G}_{i j}^{R}(\omega, \mathbf{q})=\sigma_{i} \sigma_{j} \tilde{G}_{i j}^{R}(-\omega, \mathbf{q})^{*} .
$$

Esto restringe el espectro de QNMs. Tomando por ejemplo una función de Green diagonal con $i=j$ y escribiéndola como una suma sobre todos las frecuencias quasinormales $[118,139]$ uno puede ver que las frecuencias quasinormales deben venir ya sea en pares que obedezcan $\omega_{n}$ y $\tilde{\omega}_{n}=-\omega_{n}^{*}$ o confinadas a yacer en el eje imaginario. Los residuos de los pares están relacionados mediante conjugación compleja y los puramente imaginarios tienen que tener un residuo puramente imaginario.

\section{A.2. Resolviendo las ecuaciones para las fluctuaciones}

El sector (1) - (2) del modelo gaugeado en al fase rota consiste de un sistema de ecuaciones acopladas (4.83)-(4.90). Con el fin de extraer el espectro de modos quasinormales hicimos uso de las técnicas detalladas en [100, 119], don de un método para calcular los polos de las funciones de Green en términos de campos que no son invariantes de gauge fue desarrollado. Las frecuencias quasinormales vienen dadas por el determinante de la matriz de campos generada por el conjunto mínimo de soluciones linealmente independientes que satisfacen condiciones de borde entrantes en el horizonte.

$\mathrm{Al}$ imponer condiciones de contorno entrantes, el comportamiento cerca del horizonte de los campos es

$$
\begin{aligned}
\alpha & =(\rho-1)^{\kappa}\left(\alpha_{(0)}+\alpha_{(1)}(\rho-1)+\ldots\right), \\
\beta & =(\rho-1)^{\kappa}\left(\beta_{(0)}+\beta_{(1)}(\rho-1)+\ldots\right), \\
a_{t}^{(i)} & =(\rho-1)^{\kappa+1}\left(a_{t(0)}^{(i)}+a_{t(1)}^{(i)}(\rho-1)+\ldots\right), \\
a_{x}^{(i)} & =(\rho-1)^{\kappa}\left(a_{x(0)}^{(i)}+a_{x(1)}^{(i)}(\rho-1)+\ldots\right),
\end{aligned}
$$

donde $\kappa=-i \omega / 3$ y $i=1,2$. Como el sistema está sujeto a dos restricciones, sólo tenemos libertad para elegir cuatro de los seis parámetros en el horizonte. Sin pérdida de generalidad, las soluciones pueden ser parametrizadas por $\left\{\alpha_{(0)}, \beta_{(0)}, a_{x(0)}^{(i)}\right\}$. De esta manera es posible construir cuatro soluciones distintas a las ecuaciones de movimiento que podemos etiquetar con los números $I, I I, I I I, I V$.

Dos soluciones más, $V, V I$, pueden ser obtenidas haciendo transformaciones de gauge sobre la solución trivial,

$$
\begin{aligned}
& \alpha \rightarrow 0, \beta \rightarrow i \frac{\lambda_{1} \Psi}{2}, a_{x}^{(1)} \rightarrow-k \lambda_{1}, a_{x}^{(2)} \rightarrow 0, a_{t}^{(1)} \rightarrow \omega \lambda_{1}, a_{t}^{(2)} \rightarrow i \Theta \lambda_{1}, \\
& \alpha \rightarrow i \frac{\lambda_{2} \Psi}{2}, \beta \rightarrow 0, a_{x}^{(1)} \rightarrow 0, a_{x}^{(2)} \rightarrow-k \lambda_{2}, a_{t}^{(1)} \rightarrow-i \Theta \lambda_{2}, a_{t}^{(2)} \rightarrow \omega \lambda_{2},
\end{aligned}
$$

donde $\lambda_{i}$ son constantes arbitrarias. Nótese que estas coordenadas puramente de gauge no son sólo algebraicas ya que tienen una dependencia no trivial en la coordenada del bulk $\rho$. 
La solución más general para cada campo $\varphi_{i}=\left\{\tilde{\alpha}, \tilde{\beta}, a_{t}^{(i)}, a_{x}^{(i)}\right\}$ viene dada por una combinación lineal de las soluciones de más arriba, incluyendo los modos puramente de gauge,

$$
\varphi_{i}=c_{I} \varphi_{i}^{I}+c_{I I} \varphi_{i}^{I I}+c_{I I I} \varphi_{i}^{I I}+c_{I V} \varphi_{i}^{I V}+c_{V} \varphi_{i}^{V}+c_{V I} \varphi_{i}^{V I}
$$

donde hemos definido $\{\tilde{\alpha}(\rho), \tilde{\beta}(\rho)\}=\{\rho \alpha(\rho), \rho \beta(\rho)\}$. Esta elección conveniente nos permite identificar los valores asintóticos de borde $\varphi_{i}$ con las fuentes de los operadores en la teoría de campos dual.

Como fue mostrado en [100], los polos de la función de Green retardada van a estar dados por los valores de la frecuencia para los cuales el determinante de la matriz generada por $\varphi_{i}^{N}$ se anule asintóticamente. Expandiendo el determinante y evaluándolo en un cutoff $\rho=\Lambda$,

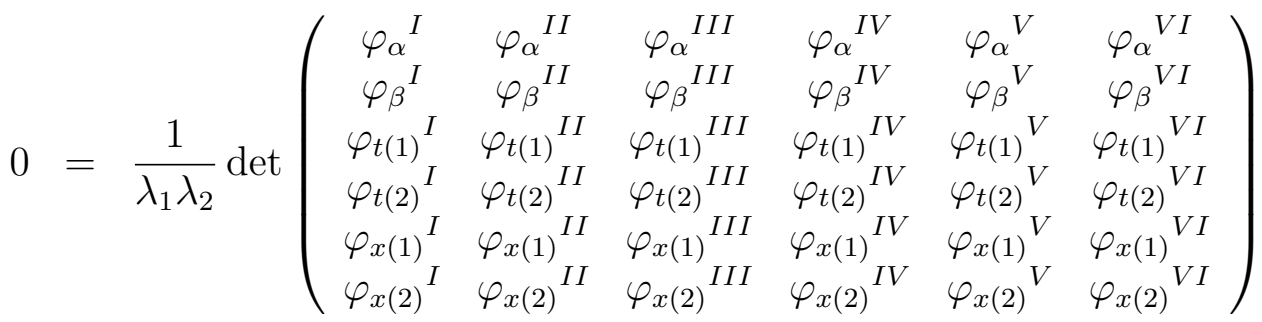

$$
\begin{aligned}
& =\omega^{2} \operatorname{det}\left(\begin{array}{cccc}
\varphi_{\alpha}^{I} & \varphi_{\alpha}^{I I} & \varphi_{\alpha}^{I I I} & \varphi_{\alpha}^{I V} \\
\varphi_{\beta}^{I} & \varphi_{\beta}^{I I} & \varphi_{\beta}^{I I I} & \varphi_{\beta}^{I V} \\
\varphi_{x(1)}^{I I} & \varphi_{x(1)}^{I I} & \varphi_{x(1)}^{I I I} & \varphi_{x(1)}^{I V} \\
\varphi_{x(2)}^{I} & \varphi_{x(2)}^{I I} & \varphi_{x(2)}^{I I I} & \varphi_{x(2)}^{I V}
\end{array}\right)+\omega k \operatorname{det}\left(\begin{array}{cccc}
\varphi_{\alpha}^{I} & \varphi_{\alpha}^{I I} & \varphi_{\alpha}^{I I I} & \varphi_{\alpha}^{I V} \\
\varphi_{\beta}^{I} & \varphi_{\beta}^{I I} & \varphi_{\beta}^{I I I} & \varphi_{\beta}^{I V} \\
\varphi_{t(1)}^{I I} & \varphi_{t(1)}^{I I} & \varphi_{t(1)}^{I I I} & \varphi_{t(1)}^{I V} \\
\varphi_{x(2)}^{I} & \varphi_{x(2)}^{I I} & \varphi_{x(2)}^{I I I} & \varphi_{x(2)}^{I V}
\end{array}\right) \\
& -\omega k \operatorname{det}\left(\begin{array}{cccc}
\varphi_{\alpha}^{I} & \varphi_{\alpha}^{I I} & \varphi_{\alpha}^{I I I} & \varphi_{\alpha}^{I V} \\
\varphi_{\beta}^{I} & \varphi_{\beta}^{I I} & \varphi_{\beta}^{I I I} & \varphi_{\beta}^{I V} \\
\varphi_{t(2)}^{I I} & \varphi_{t(2)}^{I I} & \varphi_{t(2)}^{I I I} & \varphi_{t(2)}^{I V} \\
\varphi_{x(1)}^{I} & \varphi_{x(1)}^{I I} & \varphi_{x(1)}^{I I I} & \varphi_{x(1)}^{I V}
\end{array}\right)+k^{2} \operatorname{det}\left(\begin{array}{cccc}
\varphi_{\alpha}^{I} & \varphi_{\alpha}^{I I} & \varphi_{\alpha}^{I I I} & \varphi_{\alpha}^{I V} \\
\varphi_{\beta}^{I} & \varphi_{\beta}^{I I} & \varphi_{\beta}^{I I I} & \varphi_{\beta}^{I V} \\
\varphi_{t(1)}^{I V} & \varphi_{t(1)}^{I I} & \varphi_{t(1)}^{I I} & \varphi_{t(1)}^{I V} \\
\varphi_{t(2)}^{I} & \varphi_{t(2)}^{I I} & \varphi_{t(2)}^{I I I} & \varphi_{t(2)}^{I V}
\end{array}\right),
\end{aligned}
$$

donde las condiciones de borde del background $\Theta(\Lambda)=0$ y $\Lambda \Psi=0$ ya fueron impuestas. Esta ausencia de fuentes del background para los operadores correspondientes hacen que el punto $(\omega, k)=(0,0)$ sea una solución trivial para la condición de que el determinante se anule, lo cual asegura la existencia de un modo hidrodinámico. Nótese también que el punto $(\omega, k)=(0,0)$ es una solución doble del determinante anterior.

Soluciones a las ecuaciones de movimiento y a la condición para el determinante (A.22) fueron calculadas numéricamente. Se ha corroborado que la elección de bases para la solución, i.e. de los valores iniciales de los parámetros libres, no afecta el resultado. 


\section{Apéndice B}

\section{Sobre el criterio de Landau}

\section{B.1. Ecuaciones para las fluctuaciones del sector $(0)-(3)$}

Las fluctuaciones de la teoría $U(1)$ o del sector $(0)-(3)$ contienen al sector cero y tres de los campos de gauge y a la componente inferior del doblete escalar $\sigma=\rho+i \delta$. La ecuaciones de movimiento para fluctuaciones con una dirección arbitraria del momento entonces son

$$
\begin{array}{rl}
0= & f \rho^{\prime \prime}+\left(f^{\prime}+\frac{2 f}{r}\right) \rho^{\prime}+\left(\frac{\omega^{2}}{f}+\frac{A_{0}^{2}}{f}-\frac{A_{x}^{2}}{r^{2}}-\frac{|k|^{2}}{r^{2}}-m^{2}\right) \rho+\frac{2 i \omega A_{0}}{f} \delta+2 a_{t}^{(-)} \Psi \frac{A_{0}}{f} \\
& -2 \frac{a_{x}^{(-)}}{r^{2}} \Psi A_{x}+|k| \cos (\gamma) \frac{2 i}{r^{2}} A_{x} \delta \\
0= & f \delta^{\prime \prime}+\left(f^{\prime}+\frac{2 f}{r}\right) \delta^{\prime}+\left(\frac{\omega^{2}}{f}+\frac{A_{0}^{2}}{f}-\frac{A_{x}^{2}}{r^{2}}-\frac{|k|^{2}}{r^{2}}-m^{2}\right) \delta-\frac{2 i \omega A_{0}}{f} \rho-i \Psi \omega \frac{a_{t}^{(-)}}{f} \\
& -|k| \cos (\gamma) \frac{2 i}{r^{2}} A_{x} \rho-|k| \cos (\gamma) \frac{i}{r^{2}} \Psi a_{x}^{(-)}-|k| \sin (\gamma) \frac{i}{r^{2}} \Psi a_{y}^{(-)}, \\
0= & f a_{t}^{\prime \prime(-)}+\frac{2 f}{r} a_{t}^{(-)}-\left(\frac{|k|^{2}}{r^{2}}+2 \Psi^{2}\right) a_{t}^{(-)}-\frac{\omega|k|}{r^{2}} \cos (\gamma) a_{x}^{(-)}-\frac{\omega|k|}{r^{2}} \sin (\gamma) a_{y}^{(-)} \\
& -4 \Psi A_{0} \rho-2 i \omega \Psi \delta, \\
0 & f a_{x}^{\prime \prime(-)}+f^{\prime} a_{x}^{(-)}+\left(\frac{\omega^{2}}{f}-2 \Psi^{2}\right) a_{x}^{(-)}+\frac{\omega|k|}{f} \cos (\gamma) a_{t}^{(-)}+2 i|k| \cos (\gamma) \Psi \delta \\
& -4 \Psi \rho A_{x}-\frac{|k|^{2} \sin ^{2}(\gamma)}{r^{2}} a_{x}^{(-)}+\frac{|k|^{2} \cos (\gamma) \sin (\gamma)}{r^{2}} a_{y}^{(-)} \\
0= & f a_{y}^{\prime \prime(-)}+f^{\prime} a_{y}^{(-)}+\left(\frac{\omega^{2}}{f}-2 \Psi^{2}\right) a_{y}^{(-)}+\frac{\omega|k|}{f} \sin (\gamma) a_{t}^{(-)}+2 i|k| \sin (\gamma) \Psi \delta \\
& -\frac{|k|^{2} \cos ^{2}(\gamma)}{r^{2}} a_{y}^{(-)}+\frac{|k|^{2} \cos (\gamma) \sin (\gamma)}{r^{2}} a_{x}^{(-)},
\end{array}
$$

and the constraint

$$
0=\frac{i \omega}{f} a_{t}^{\prime(-)}+\frac{i|k|}{r^{2}} \cos (\gamma) a_{x}^{\prime(-)}+\frac{i|k|}{r^{2}} \sin (\gamma) a_{y}^{\prime(-)}+2 \Psi^{\prime} \delta-2 \Psi \delta^{\prime}
$$


where we have used $k_{x}=|k| \cos (\gamma), k_{y}=|k| \sin (\gamma)$. The general pure gauge solution in this sector is

$$
\delta=i \lambda \Psi ; \quad \rho=0 ; \quad a_{t}^{(-)}=\lambda \omega ; \quad a_{x}^{(-)}=-\lambda|k| \cos (\gamma) ; \quad a_{y}^{(-)}=-\lambda|k| \sin (\gamma)
$$

donde $\lambda$ es una constante arbitraria.

\section{B.2. Ecuaciones para las fluctuaciones en el sector (1)-(2)}

Las perturbaciones en el sector $(1)-(2)$ de la teoría $U(2)$ incluyen las fluctuaciones para la componente superior del doblete escalar, $\eta=\alpha+i \beta$, junto con los correspondientes campos de gauge. Para momento en la dirección opuesta a la velocidad de superfluido, las 
ecuaciones de movimiento son

$$
\begin{aligned}
& 0=f a_{x}^{\prime \prime(1)}+f^{\prime} a_{x}^{\prime(1)}+\left(\frac{\omega^{2}}{f}-\Psi^{2}+\frac{\left(A_{t}^{(3)}\right)^{2}}{f}\right) a_{x}^{(1)}-2 i \frac{A_{t}^{(3)} \omega}{f} a_{x}^{(2)}+i \omega \frac{A_{x}^{(3)}}{f} a_{t}^{(2)} \\
& -\frac{A_{t}^{(3)} A_{x}^{(3)}}{f} a_{t}^{(1)}-2 A_{x}^{(0)} \Psi \alpha+2 i k \Psi \beta-\frac{i k A_{t}^{(3)}}{f} a_{t}^{(2)}+\frac{\omega k}{f} a_{t}^{(1)}, \\
& 0=f a_{x}^{\prime \prime(2)}+f^{\prime} a_{x}^{\prime(2)}+\left(\frac{\omega^{2}}{f}-\Psi^{2}+\frac{\left(A_{t}^{(3)}\right)^{2}}{f}\right) a_{x}^{(2)}+2 i \frac{A_{t}^{(3)} \omega}{f} a_{x}^{(1)}-i \omega \frac{A_{x}^{(3)}}{f} a_{t}^{(1)} \\
& -\frac{A_{t}^{(3)} A_{x}^{(3)}}{f} a_{t}^{(2)}+2 \Psi A_{x}^{(0)} \beta+2 i k \Psi \alpha+\frac{i k A_{t}^{(3)}}{f} a_{t}^{(1)}+\frac{\omega k}{f} a_{t}^{(2)}, \\
& 0=f a_{t}^{\prime \prime(1)}+\frac{2 f}{r} a_{t}^{\prime(1)}-\left(\frac{\left(A_{x}^{(3)}\right)^{2}}{r^{2}}+\Psi^{2}+\frac{k^{2}}{r^{2}}\right) a_{t}^{(1)}+\frac{A_{t}^{(3)} A_{x}^{(3)}}{r^{2}} a_{x}^{(1)}-i \omega \frac{A_{x}^{(3)}}{r^{2}} a_{x}^{(2)}-2 i \omega \beta \Psi \\
& -2 \phi \Psi \alpha+\frac{i k A_{t}^{(3)}}{r^{2}} a_{x}^{(2)}-\frac{2 i k A_{x}^{(3)}}{r^{2}} a_{t}^{(2)}-\frac{\omega k}{r^{2}} a_{x}^{(1)}, \\
& 0=f a_{t}^{\prime \prime(2)}+\frac{2 f}{r} a_{t}^{\prime(2)}-\left(\frac{\left(A_{x}^{(3)}\right)^{2}}{r^{2}}+\Psi^{2}+\frac{k^{2}}{r^{2}}\right) a_{t}^{(2)}+\frac{A_{t}^{(3)} A_{x}^{(3)}}{r^{2}} a_{x}^{(2)}+i \omega \frac{A_{x}^{(3)}}{r^{2}} a_{x}^{(1)} \\
& -\frac{i k A_{t}^{(3)}}{r^{2}} a_{x}^{(1)}+\frac{2 i k A_{x}^{(3)}}{r^{2}} a_{t}^{(1)}-\frac{\omega k}{r^{2}} a_{x}^{(2)}-2 i \omega \alpha \Psi+2 A_{t}^{(0)} \Psi \beta, \\
& 0=f \alpha^{\prime \prime}+\left(f^{\prime}+\frac{2 f}{r}\right) \alpha^{\prime}+\left(\frac{\omega^{2}}{f}+\frac{\left(A_{t}^{(0)}+A_{t}^{(3)}\right)^{2}}{4 f}-\frac{\left(A_{x}^{(0)}+A_{x}^{(3)}\right)^{2}}{4 r^{2}}-\frac{k^{2}}{r^{2}}-m^{2}\right) \alpha \\
& +\left(i \omega\left(\frac{A_{t}^{(0)}+A_{t}^{(3)}}{f}\right)+\frac{i k}{r^{2}}\left(A_{x}^{(0)}+A_{x}^{(3)}\right)\right) \beta+\frac{A_{t}^{(0)} \Psi}{2 f} a_{t}^{(1)}-i \omega \frac{\Psi}{2 f} a_{t}^{(2)} \\
& -\frac{A_{x}^{(0)} \Psi}{2 r^{2}} a_{x}^{(1)}-\frac{i k \Psi}{2 r^{2}} a_{x}^{(2)}, \\
& 0=f \beta^{\prime \prime}+\left(f^{\prime}+\frac{2 f}{r}\right) \beta^{\prime}+\left(\frac{\omega^{2}}{f}+\frac{\left(A_{t}^{(0)}+A_{t}^{(3)}\right)^{2}}{4 f}-\frac{\left(A_{x}^{(0)}+A_{x}^{(3)}\right)^{2}}{4 r^{2}}-\frac{k^{2}}{r^{2}}-m^{2}\right) \beta \\
& -\left(i \omega\left(\frac{A_{t}^{(0)}+A_{t}^{(3)}}{f}\right)+\frac{i k}{r^{2}}\left(A_{x}^{(0)}+A_{x}^{(3)}\right)\right) \alpha-\frac{A_{t}^{(0)} \Psi}{2 f} a_{t}^{(2)}-i \omega \frac{\Psi}{2 f} a_{t}^{(1)} \\
& +\frac{A_{x}^{(0)} \Psi}{2 r^{2}} a_{x}^{(2)}-\frac{i k \Psi}{2 r^{2}} a_{x}^{(1)}
\end{aligned}
$$


sujetas a las restricciones

$$
\begin{aligned}
& 0=2 f\left(\Psi \beta^{\prime}-\Psi^{\prime} \beta\right)+a_{t}^{(2)} A_{t}^{\prime(3)}-a_{t}^{\prime(2)} A_{t}^{(3)}+\frac{f}{r^{2}}\left(A_{x}^{(3)} a_{x}^{\prime(2)}-a_{x}^{(2)} A_{x}^{\prime(3)}\right)-i \omega a_{t}^{\prime(1)}-\frac{i k f}{r^{2}} a_{x}^{\prime(1)}, \\
& 0=2 f\left(\Psi \alpha^{\prime}-\Psi^{\prime} \alpha\right)+a_{t}^{\prime(1)} A_{t}^{(3)}-a_{t}^{(1)} A_{t}^{\prime(3)}+\frac{f}{r^{2}}\left(a_{x}^{(1)} A_{x}^{\prime(3)}-A_{x}^{(3)} a_{x}^{\prime(1)}\right)-i \omega a_{t}^{\prime(2)}-\frac{i k f}{r^{2}} a_{x}^{\prime(2)},
\end{aligned}
$$

Hay dos soluciones puramente de gauge en este sector,

$$
\begin{gathered}
\alpha=0, \quad \beta=i \lambda_{1} \Psi / 2, \quad a_{t}^{(1)}=\lambda_{1} \omega, \quad a_{t}^{(2)}=i \lambda_{1} A_{t}^{(3)}, \quad a_{x}^{(1)}=-\lambda_{1} k, \quad a_{x}^{(2)}=i \lambda_{1} A_{x}^{(3)}, \\
\alpha=i \lambda_{2} \Psi / 2, \quad \beta=0, \quad a_{t}^{(1)}=-i \lambda_{2} A_{t}^{(3)}, \quad a_{t}^{(2)}=\lambda_{2} \omega, \quad a_{x}^{(1)}=-i \lambda_{2} A_{x}^{(3)}, \quad a_{x}^{(2)}=-\lambda_{2} k,
\end{gathered}
$$

donde $\lambda_{1}$ y $\lambda_{2}$ son constantes arbitrarias. 


\section{Bibliografía}

[1] S. A. Hartnoll, "Lectures on holographic methods for condensed matter physics," Class. Quant. Grav. 26 (2009) 224002 [arXiv:0903.3246 [hep-th]].

[2] C. P. Herzog, "Lectures on Holographic Superfluidity and Superconductivity," J. Phys. A 42 (2009) 343001 [arXiv:0904.1975 [hep-th]].

[3] J. M. Maldacena, "The large N limit of superconformal field theories and supergravity," Adv. Theor. Math. Phys. 2 (1998) 231 [Int. J. Theor. Phys. 38 (1999) 1113] [arXiv:hepth/9711200].

[4] E. Witten, "Anti-de Sitter space and holography," Adv. Theor. Math. Phys. 2 (1998) 253 [hep-th/9802150].

[5] S. S. Gubser, I. R. Klebanov and A. M. Polyakov, "Gauge theory correlators from noncritical string theory," Phys. Lett. B 428 (1998) 105 [hep-th/9802109].

[6] T. Sakai and S. Sugimoto, "Low energy hadron physics in holographic QCD," Prog. Theor. Phys. 113 (2005) 843 [hep-th/0412141].

[7] J. Erlich, E. Katz, D. T. Son and M. A. Stephanov, "QCD and a holographic model of hadrons," Phys. Rev. Lett. 95 (2005) 261602 [hep-ph/0501128].

[8] D. Belitz, T. R. Kirkpatrick, "Quantum phase transitions," [cond-mat/9811058].

[9] M. Mulligan, C. Nayak and S. Kachru, "An Isotropic to Anisotropic Transition in a Fractional Quantum Hall State," Phys. Rev. B 82 (2010) 085102 [arXiv:1004.3570 [condmat.str-el]].

[10] N. Grandi, I. S. Landea and G. A. Silva, "Vortex solutions of the Lifshitz-Chern-Simons theory," Phys. Rev. D 87 (2013) 025031 [arXiv:1206.0611 [cond-mat.str-el]].

[11] P. Philips, "Fractionalize this," Nature Physics 6931 Ü933 (2010)

[12] N. Iqbal, H. Liu and M. Mezei, "Lectures on holographic non-Fermi liquids and quantum phase transitions," arXiv:1110.3814 [hep-th].

[13] H. Liu, J. McGreevy and D. Vegh, "Non-Fermi liquids from holography," Phys. Rev. D 83 (2011) 065029 [arXiv:0903.2477 [hep-th]].

[14] T. Faulkner, H. Liu, J. McGreevy and D. Vegh, "Emergent quantum criticality, Fermi surfaces, and AdS(2)," Phys. Rev. D 83 (2011) 125002 [arXiv:0907.2694 [hep-th]]. 
[15] M. Cubrovic, J. Zaanen and K. Schalm, "String Theory, Quantum Phase Transitions and the Emergent Fermi-Liquid," Science 325 (2009) 439 [arXiv:0904.1993 [hep-th]].

[16] T. Faulkner, N. Iqbal, H. Liu, J. McGreevy and D. Vegh, "From Black Holes to Strange Metals," arXiv:1003.1728 [hep-th].

[17] T. Faulkner, N. Iqbal, H. Liu, J. McGreevy and D. Vegh, "Strange metal transport realized by gauge/gravity duality," Science 329 (2010) 1043.

[18] O. Aharony, S. S. Gubser, J. M. Maldacena, H. Ooguri and Y. Oz, "Large N field theories, string theory and gravity," Phys. Rept. 323 (2000) 183 [hep-th/9905111].

[19] A. Zaffaroni, "Introduction to the AdS-CFT correspondence," Class. Quant. Grav. 17 (2000) 3571.

[20] S. Kachru, X. Liu and M. Mulligan, "Gravity Duals of Lifshitz-like Fixed Points," Phys. Rev. D 78 (2008) 106005 [arXiv:0808.1725 [hep-th]].

[21] K. Skenderis, "Lecture notes on holographic renormalization," Class. Quant. Grav. 19 (2002) 5849 [hep-th/0209067].

[22] M. Henningson and K. Sfetsos, "Spinors and the AdS / CFT correspondence," Phys. Lett. B 431 (1998) 63 [hep-th/9803251].

[23] W. Mueck and K. S. Viswanathan, "Conformal field theory correlators from classical field theory on anti-de Sitter space. 2. Vector and spinor fields," Phys. Rev. D 58 (1998) 106006 [hep-th/9805145].

[24] N. Iqbal and H. Liu, "Real-time response in AdS/CFT with application to spinors," Fortsch. Phys. 57 (2009) 367 [arXiv:0903.2596 [hep-th]].

[25] D. Arean, A. Farahi, L. A. Pando Zayas, I. S. Landea and A. Scardicchio, "A Dirty Holographic Superconductor," arXiv:1308.1920 [hep-th].

[26] S. S. Gubser, "Breaking an Abelian gauge symmetry near a black hole horizon," Phys. Rev. D 78 (2008) 065034 [arXiv:0801.2977 [hep-th]].

[27] S. A. Hartnoll, C. P. Herzog and G. T. Horowitz, "Building a Holographic Superconductor," Phys. Rev. Lett. 101 (2008) 031601 [arXiv:0803.3295 [hep-th]].

[28] S. A. Hartnoll, C. P. Herzog and G. T. Horowitz, "Holographic Superconductors," JHEP 0812 (2008) 015 [arXiv:0810.1563 [hep-th]].

[29] S. S. Gubser and S. S. Pufu, "The Gravity dual of a p-wave superconductor," JHEP 0811 (2008) 033 [arXiv:0805.2960 [hep-th]].

[30] J. Cardy, " Scaling and Renormalization in Statistical Physics" .

[31] P. Phillips, " Advanced Solid State Physics", (Cambridge University Press, Cambridge 2012) 
[32] D. Basko, I. Aleiner and B. Altshuler, "Metal insulator transition in a weakly interacting many-electron system with localized single-particle states,. ${ }^{\mathrm{A}}$ nnals of Physics 321 (2006) 1126.

[33] A. Pal, D.Huse, "Many-body localization phase transition", Physical Review B, 82, (2010) 174411.

[34] Shankar Iyer, Vadim Oganesyan, Gil Refael, and David A. Huse, Phys. Rev. B 87, 134202 (2013).

[35] A.De Luca and A.Scardicchio, EPL 10137003 (2013).

[36] F. Buccheri, A. De Luca and A. Scardicchio, "Structure of typical states of a disordered Richardson model and many-body localization", Phys. Rev. B, 84 (2011) 094203.

[37] S. A. Hartnoll, P. K. Kovtun, M. Muller and S. Sachdev, "Theory of the Nernst effect near quantum phase transitions in condensed matter, and in dyonic black holes," Phys. Rev. B 76 (2007) 144502 [arXiv:0706.3215 [cond-mat.str-el]].

[38] S. A. Hartnoll and C. P. Herzog, "Impure AdS/CFT correspondence," Phys. Rev. D 77 (2008) 106009 [arXiv:0801.1693 [hep-th]].

[39] M. Fujita, Y. Hikida, S. Ryu and T. Takayanagi, "Disordered Systems and the Replica Method in AdS/CFT," JHEP 0812 (2008) 065 [arXiv:0810.5394 [hep-th]].

[40] S. Ryu, T. Takayanagi and T. Ugajin, "Holographic Conductivity in Disordered Systems," JHEP 1104 (2011) 115 [arXiv:1103.6068 [hep-th]].

[41] A. Adams and S. Yaida, "Disordered Holographic Systems I: Functional Renormalization," arXiv:1102.2892 [hep-th].

[42] A. Adams and S. Yaida, "Disordered Holographic Systems II: Marginal Relevance of Imperfection," arXiv:1201.6366 [hep-th].

[43] O. Saremi, "Disorder in Gauge/Gravity Duality, Pole Spectrum Statistics and Random Matrix Theory," arXiv:1206.1856 [hep-th].

[44] P. Anderson, "Theory of dirty superconductors," Journal of Physics and Chemistry of Solids, vol. 11, 26.

[45] S.Maekawa, H.Fukuyama, Journal of the Physical Society of Japan, 511380 (1981).

[46] A. Kapitulnik, G. Kotliar, Phys. Rev. Lett. 54, 473, (1985).

G. Kotliar, A. Kapitulnik, Phys. Rev. B 33, 3146 (1986).

[47] M. Ma, P.A. Lee, Phys. Rev. B 32, 5658, (1985).

[48] G. T. Horowitz, "Theory of Superconductivity," Lect. Notes Phys. 828 (2011) 313 [arXiv:1002.1722 [hep-th]].

[49] M. Chand et al., "Phase diagram of the strongly disordered s-wave superconductor NbN close to the metal-insulator transition," Phys. Rev. D 85 (2012) 014508 
[50] G. T. Horowitz, J. E. Santos, D. Tong and , "Optical Conductivity with Holographic Lattices," JHEP 1207 (2012) 168 [arXiv:1204.0519 [hep-th]].

[51] R. Flauger, E. Pajer and S. Papanikolaou, "A Striped Holographic Superconductor," Phys. Rev. D 83 (2011) 064009 [arXiv:1010.1775 [hep-th]].

[52] G. T. Horowitz and J. E. Santos, "General Relativity and the Cuprates," arXiv:1302.6586 [hep-th].

[53] D. Fisher, M. P. Fisher and D. Huse, "Thermal fluctuations, quenched disorder, phase transitions, and transport in type-II superconductors," Phys. Rev. B 43 (1991) 130.

[54] N. Bao, X. Dong, E. Silverstein and G. Torroba, "Stimulated superconductivity at strong coupling," JHEP 1110 (2011) 123 [arXiv:1104.4098 [hep-th]].

[55] M. Natsuume and T. Okamura, "The enhanced holographic superconductor: is it possible?," arXiv:1307.6875 [hep-th].

[56] W.-J. Li, Y. Tian and H. Zhang, "Periodically Driven Holographic Superconductor," JHEP 1307 (2013) 030 [arXiv:1305.1600 [hep-th]].

[57] J. Erdmenger, X.-H. Ge and D.-W. Pang, "Striped phases in the holographic insulator/superconductor transition," arXiv:1307.4609 [hep-th].

[58] S. K. Domokos, C. Hoyos and J. Sonnenschein, "Stability conditions for spatially modulated phases," arXiv:1307.3773 [hep-th].

[59] S. A. Hartnoll and D. M. Hofman, "Locally Critical Resistivities from Umklapp Scattering," Phys. Rev. Lett. 108 (2012) 241601 [arXiv:1201.3917 [hep-th]].

[60] R. E. Arias and I. S. Landea, "Backreacting p-wave Superconductors," JHEP 1301 (2013) 157 [arXiv:1210.6823 [hep-th]].

[61] R. E. Arias, "AdS/CFT and its Applications," arXiv:1305.1917 [hep-th].

[62] S. Ryu and T. Takayanagi, "Holographic derivation of entanglement entropy from AdS/CFT," Phys. Rev. Lett. 96 (2006) 181602, hep-th/0603001.

[63] S. Ryu and T. Takayanagi, "Aspects of holographic entanglement entropy," JHEP 0608 (2006) 045, hep-th/0605073.

[64] V. E. Hubeny, M. Rangamani and T. Takayanagi, "A covariant holographic entanglement entropy proposal," JHEP 0707 (2007) 062, 0705.0016 [hep-th].

[65] T. Nishioka, S. Ryu and T. Takayanagi, "Holographic Entanglement Entropy: An Overview," J. Phys. A 42 (2009) 504008, arXiv:0905.0932 [hep-th].

[66] M. Ammon, J. Erdmenger, V. Grass, P. Kerner and A. O’Bannon, "On Holographic p-wave Superfluids with Back-reaction," Phys. Lett. B 686 (2010) 192 [arXiv:0912.3515 [hep-th]]. 
[67] R.-G. Cai, Z.-Y. Nie and H.-Q. Zhang, "Holographic Phase Transitions of P-wave Superconductors in Gauss-Bonnet Gravity with Back-reaction," Phys. Rev. D 83 (2011) 066013 [arXiv:1012.5559 [hep-th]].

[68] T. Takayanagi, "Entanglement Entropy from a Holographic Viewpoint," Class. Quant. Grav. 29 (2012) 153001 [arXiv:1204.2450 [gr-qc]].

[69] T. Albash and C. V. Johnson, "Holographic Studies of Entanglement Entropy in Superconductors," JHEP 1205 (2012) 079 [arXiv:1202.2605 [hep-th]].

[70] R.-G. Cai, S. He, L. Li and Y.-L. Zhang, "Holographic Entanglement Entropy in Insulator/Superconductor Transition," JHEP 1207 (2012) 088 [arXiv:1203.6620 [hep-th]].

[71] R.-G. Cai, S. He, L. Li and Y.-L. Zhang, "Holographic Entanglement Entropy on P-wave Superconductor Phase Transition," JHEP 1207 (2012) 027 [arXiv:1204.5962 [hep-th]].

[72] R.-G. Cai, S. He, L. Li and L.-F. Li, "Entanglement Entropy and Wilson Loop in Stúckelberg Holographic Insulator/Superconductor Model," arXiv:1209.1019 [hep-th].

[73] M. Ammon, J. Erdmenger, M. Kaminski and P. Kerner, "Superconductivity from gauge/gravity duality with flavor," Phys. Lett. B 680 (2009) 516 [arXiv:0810.2316 [hep-th]].

[74] P. Basu, J. He, A. Mukherjee and H.-H. Shieh, "Superconductivity from D3/D7: Holographic Pion Superfluid," JHEP 0911 (2009) 070 [arXiv:0810.3970 [hep-th]].

[75] M. Ammon, J. Erdmenger, M. Kaminski and P. Kerner, "Flavor Superconductivity from Gauge/Gravity Duality," JHEP 0910 (2009) 067 [arXiv:0903.1864 [hep-th]].

[76] K. Peeters, J. Powell and M. Zamaklar, "Exploring colourful holographic superconductors," JHEP 0909 (2009) 101 [arXiv:0907.1508 [hep-th]].

[77] M. Ammon, "Gauge/gravity duality applied to condensed matter systems," Fortsch. Phys. 58 (2010) 1123 .

[78] M. Kaminski, "Flavor Superconductivity and Superfluidity," Lect. Notes Phys. 828 (2011) 349 [arXiv:1002.4886 [hep-th]].

[79] S. A. Hartnoll, "Horizons, holography and condensed matter," arXiv:1106.4324 [hep-th].

[80] H. Casini, M. Huerta and R. C. Myers, "Towards a derivation of holographic entanglement entropy," JHEP 1105 (2011) 036 [arXiv:1102.0440 [hep-th]].

[81] S. Gangopadhyay and D. Roychowdhury, "Analytic study of properties of holographic p-wave superconductors," JHEP 1208 (2012) 104 [arXiv:1207.5605 [hep-th]].

[82] E. Winstanley, "Classical Yang-Mills black hole hair in anti-de Sitter space," Lect. Notes Phys. 769 (2009) 49 [arXiv:0801.0527 [gr-qc]].

[83] R. Manvelyan, E. Radu and D. H. Tchrakian, "New AdS non Abelian black holes with superconducting horizons," Phys. Lett. B 677 (2009) 79 [arXiv:0812.3531 [hep-th]]. 
[84] I. R. Klebanov and E. Witten, "AdS / CFT correspondence and symmetry breaking," Nucl. Phys. B 556 (1999) 89 [hep-th/9905104].

[85] S. J. Rey and J. T. Yee, "Macroscopic strings as heavy quarks in large N gauge theory and anti-de Sitter supergravity," Eur. Phys. J. C 22 (2001) 379 [arXiv:hep-th/9803001].

[86] J. M. Maldacena, "Wilson loops in large N field theories," Phys. Rev. Lett. 80, 4859 (1998) [arXiv:hep-th/9803002].

[87] I. R. Klebanov, D. Kutasov and A. Murugan, "Entanglement as a probe of confinement," Nucl. Phys. B 796 (2008) 274 [arXiv:0709.2140 [hep-th]].

[88] I. Bah, A. Faraggi, L. A. Pando Zayas and C. A. Terrero-Escalante, "Holographic entanglement entropy and phase transitions at finite temperature," Int. J. Mod. Phys. A 24 (2009) 2703 [arXiv:0710.5483 [hep-th]].

[89] I. Amado, D. Arean, A. Jimenez-Alba, K. Landsteiner, L. Melgar and I. S. Landea, "Holographic Type II Goldstone bosons," JHEP 1307 (2013) 108 [arXiv:1302.5641 [hepthl].

[90] F. Benini, C. P. Herzog, R. Rahman and A. Yarom, "Gauge gravity duality for d-wave superconductors: prospects and challenges," JHEP 1011 (2010) 137 [arXiv:1007.1981 [hep-th]].

[91] J.-W. Chen, Y.-J. Kao, D. Maity, W.-Y. Wen and C.-P. Yeh, "Towards A Holographic Model of D-Wave Superconductors," Phys. Rev. D 81 (2010) 106008 [arXiv:1003.2991 [hep-th]].

[92] G. T. Horowitz, "Introduction to Holographic Superconductors," arXiv:1002.1722 [hepth].

[93] M. Kaminski, "Flavor Superconductivity \& Superfluidity," Lect. Notes Phys. 828 (2011) 349 [arXiv:1002.4886 [hep-th]].

[94] D. T. Son and A. O. Starinets, "Minkowski space correlators in AdS / CFT correspondence: Recipe and applications," JHEP 0209 (2002) 042 [hep-th/0205051].

[95] C. P. Herzog and D. T. Son, "Schwinger-Keldysh propagators from AdS/CFT correspondence," JHEP 0303 (2003) 046 [hep-th/0212072].

[96] G. T. Horowitz and V. E. Hubeny, "Quasinormal modes of AdS black holes and the approach to thermal equilibrium," Phys. Rev. D 62 (2000) 024027 [hep-th/9909056].

[97] D. Birmingham, I. Sachs and S. N. Solodukhin, "Conformal field theory interpretation of black hole quasinormal modes," Phys. Rev. Lett. 88 (2002) 151301 [hep-th/0112055].

[98] E. Berti, V. Cardoso and A. O. Starinets, "Quasinormal modes of black holes and black branes," Class. Quant. Grav. 26 (2009) 163001 [arXiv:0905.2975 [gr-qc]].

[99] K. Landsteiner, "The Sound of Strongly Coupled Field Theories: Quasinormal Modes In AdS," AIP Conf. Proc. 1458 (2011) 174 [arXiv:1202.3550 [gr-qc]]. 
[100] I. Amado, M. Kaminski and K. Landsteiner, "Hydrodynamics of Holographic Superconductors," JHEP 0905 (2009) 021 [arXiv:0903.2209 [hep-th]].

[101] A. Yarom, "Fourth sound of holographic superfluids," JHEP 0907 (2009) 070 [arXiv:0903.1353 [hep-th]].

[102] M. J. Bhaseen, J. P. Gauntlett, B. D. Simons, J. Sonner and T. Wiseman, "Holographic Superfluids and the Dynamics of Symmetry Breaking," arXiv:1207.4194 [hep-th].

[103] B.I. Halperin, "Dynamic properties of the multicomponent Bose fluid,"Phys. Rev. B 11, 178âĂŞ190 (1975).

[104] T. Schafer, D. T. Son, M. A. Stephanov, D. Toublan and J. J. M. Verbaarschot, "Kaon condensation and Goldstone's theorem," Phys. Lett. B 522 (2001) 67 [hep-ph/0108210].

[105] V. A. Miransky and I. A. Shovkovy, "Spontaneous symmetry breaking with abnormal number of Nambu-Goldstone bosons and kaon condensate," Phys. Rev. Lett. 88 (2002) 111601 [hep-ph/0108178].

[106] V. G. Filev, C. V. Johnson and J. P. Shock, "Universal Holographic Chiral Dynamics in an External Magnetic Field," JHEP 0908 (2009) 013 [arXiv:0903.5345 [hep-th]].

[107] T. Brauner, "Spontaneous Symmetry Breaking and Nambu-Goldstone Bosons in Quantum Many-Body Systems," Symmetry 2 (2010) 609 [arXiv:1001.5212 [hep-th]].

[108] H. B. Nielsen and S. Chadha, "On How to Count Goldstone Bosons," Nucl. Phys. B 105 (1976) 445.

[109] H. Watanabe and T. Brauner, "On the number of Nambu-Goldstone bosons and its relation to charge densities," Phys. Rev. D 84 (2011) 125013 [arXiv:1109.6327 [hep-ph]].

[110] H. Watanabe and H. Murayama, "Unified Description of Nambu-Goldstone Bosons without Lorentz Invariance," Phys. Rev. Lett. 108 (2012) 251602 [arXiv:1203.0609 [hepthl].

[111] H. Watanabe and H. Murayama, "Redundancies in Nambu-Goldstone Bosons," arXiv:1302.4800 [cond-mat.other].

[112] Y. Hidaka, "Counting rule for Nambu-Goldstone modes in nonrelativistic systems," Phys. Rev. Lett. 110 (2013) 091601 arXiv:1203.1494 [hep-th].

[113] A. Kapustin, "Remarks on nonrelativistic Goldstone bosons," arXiv:1207.0457 [hep$\mathrm{ph}]$.

[114] Z. Q. Li, E. A. Henriksen, Z. Jiang, Z. Hao, M. C. Martin, P. Kim, H. L. Stormer and D. N. Basov, "Dirac charge dynamics in graphene by infrared spectroscopy," Nature Physics 4, 532 - 535 (2008).

[115] T. Brauner, "Spontaneous symmetry breaking in the linear sigma model at finite chemical potential: One-loop corrections," Phys. Rev. D 74 (2006) 085010 [hep-ph/0607102]. 
[116] A. Nicolis and F. Piazza, "A relativistic non-relativistic Goldstone theorem: gapped Goldstones at finite charge density," Phys. Rev. Lett. 110 (2013) 011602 [arXiv:1204.1570 [hep-th]].

[117] I. R. Klebanov and E. Witten, "AdS / CFT correspondence and symmetry breaking," Nucl. Phys. B 556 (1999) 89 [hep-th/9905104].

[118] I. Amado, C. Hoyos-Badajoz, K. Landsteiner and S. Montero, "Hydrodynamics and beyond in the strongly coupled N=4 plasma," JHEP 0807 (2008) 133 [arXiv:0805.2570 [hep-th]].

[119] M. Kaminski, K. Landsteiner, J. Mas, J. P. Shock and J. Tarrio, "Holographic Operator Mixing and Quasinormal Modes on the Brane," JHEP 1002 (2010) 021 [arXiv:0911.3610 [hep-th]].

[120] R. A. Davison and A. O. Starinets, "Holographic zero sound at finite temperature," Phys. Rev. D 85 (2012) 026004 [arXiv:1109.6343 [hep-th]].

[121] F. Bigazzi, A. L. Cotrone, D. Musso, N. P. Fokeeva and D. Seminara, "Unbalanced Holographic Superconductors and Spintronics," JHEP 1202 (2012) 078 [arXiv:1111.6601 [hep-th]].

[122] S. C. 'Zhang. 1997. " A Unified Theory Based on SO(5) Symmetry of Superconductivity and Antiferromagnetism," Science,275,1089

[123] S. Uchino, M. Kobayashi and M. Ueda, "Bogoliubov Theory and Lee-Huang-Yang Corrections in Spin-1 and Spin-2 Bose-Einstein Condensates in the Presence of the Quadratic Zeeman Effect," Phys. Rev. A 81 (2010) 063632. [arXiv:0912.0355 [condmat.quant-gas]].

[124] I. M. Khalatnikov, "An Introduction to the Theory of Superfluidity," Advanced Book Classics, Westview Press.

[125] L. .D. Landau and E. M. Lifshitz, "Course of Theoretical Physcis," Vol. 9, Statistical Physics, Part 2, Chap. III, Pergamon Press.

[126] C. Hoyos-Badajoz, K. Landsteiner and S. Montero, "Holographic meson melting," JHEP 0704 (2007) 031 [hep-th/0612169].

[127] M. Kaminski, K. Landsteiner, F. Pena-Benitez, J. Erdmenger, C. Greubel and P. Kerner, "Quasinormal modes of massive charged flavor branes," JHEP 1003 (2010) 117 [arXiv:0911.3544 [hep-th]].

[128] V. P. Gusynin, V. A. Miransky and I. A. Shovkovy, "Spontaneous rotational symmetry breaking and roton - like excitations in gauged sigma model at finite density," Phys. Lett. B 581, 82 (2004) [hep-ph/0311025].

[129] V. P. Gusynin, V. A. Miransky and I. A. Shovkovy, "Surprises in nonperturbative dynamics in sigma-model at finite density," Mod. Phys. Lett. A 19 (2004) 1341 [hep$\mathrm{ph} / 0406219]$. 
[130] M. Ammon, J. Erdmenger, M. Kaminski and P. Kerner, "Superconductivity from gauge/gravity duality with flavor," Phys. Lett. B 680 (2009) 516 [arXiv:0810.2316 [hep-th]].

[131] J. P. Gauntlett, J. Sonner and T. Wiseman, "Holographic superconductivity in MTheory," Phys. Rev. Lett. 103 (2009) 151601 [arXiv:0907.3796 [hep-th]].

[132] S. S. Gubser, C. P. Herzog, S. S. Pufu and T. Tesileanu, "Superconductors from Superstrings," Phys. Rev. Lett. 103 (2009) 141601 [arXiv:0907.3510 [hep-th]].

[133] N. Bobev, N. Halmagyi, K. Pilch and N. P. Warner, "Supergravity Instabilities of Non-Supersymmetric Quantum Critical Points," Class. Quant. Grav. 27 (2010) 235013 [arXiv:1006.2546 [hep-th]].

[134] N. Bobev, A. Kundu, K. Pilch and N. P. Warner, "Minimal Holographic Superconductors from Maximal Supergravity," JHEP 1203 (2012) 064 [arXiv:1110.3454 [hep-th]].

[135] S. Bhattacharyya, V. EHubeny, S. Minwalla and M. Rangamani, "Nonlinear Fluid Dynamics from Gravity," JHEP 0802 (2008) 045 [arXiv:0712.2456 [hep-th]].

[136] C. P. Herzog, P. K. Kovtun and D. T. Son, "Holographic model of superfluidity," Phys. Rev. D 79 (2009) 066002 [arXiv:0809.4870 [hep-th]].

[137] P. Basu, A. Mukherjee and H.-H. Shieh, "Supercurrent: Vector Hair for an AdS Black Hole," Phys. Rev. D 79 (2009) 045010 [arXiv:0809.4494 [hep-th]].

[138] D. Arean, M. Bertolini, J. Evslin and T. Prochazka, "On Holographic Superconductors with DC Current," JHEP 1007 (2010) 060 [arXiv:1003.5661 [hep-th]].

[139] M. A. Stephanov and Y. Yin, "Conductivity and quasinormal modes in holographic theories," JHEP 1202 (2012) 017 [arXiv:1111.5303 [hep-ph]].

[140] I. Amado, D. Arean, A. Jimenez-Alba, L. Melgar and I. S. Landea, "Holographic s+p Superconductors," arXiv:1309.5086 [hep-th].

[141] D. Vollhardt and P. Wolfle, The Superfluid Phases of Helium 3 (Taylor \& Francis, London, 1990).

[142] P. Goswami and B. Roy, "Axionic superconductivity in three dimensional doped narrow gap semiconductors," arXiv:1307.3240 [cond-mat.supr-con].

[143] A. P. Schnyder, S. Ryu, A. Furusaki, A. W. W. Ludwig, "Classification of topological insulators and superconductors in three spatial dimensions," Phys. Rev. B 78, 195125 (2008).

[144] J.-W. Chen, Y.-J. Kao, D. Maity, W.-Y. Wen and C.-P. Yeh, "Towards A Holographic Model of D-Wave Superconductors," Phys. Rev. D 81 (2010) 106008 [arXiv:1003.2991 [hep-th]]; F. Benini, C. P. Herzog, R. Rahman and A. Yarom, "Gauge gravity duality for d-wave superconductors: prospects and challenges," JHEP 1011 (2010) 137 [arXiv:1007.1981 [hep-th]]. 
[145] P. Basu, J. He, A. Mukherjee, M. Rozali and H.-H. Shieh, "Competing Holographic Orders," JHEP 1010 (2010) 092 [arXiv:1007.3480 [hep-th]].

[146] L. A. Pando Zayas and D. Reichmann, "A Holographic Chiral $p_{x}+i p_{y}$ Superconductor," Phys. Rev. D 85 (2012) 106012 [arXiv:1108.4022 [hep-th]].

[147] D. Musso, "Competition/Enhancement of Two Probe Order Parameters in the Unbalanced Holographic Superconductor," JHEP 1306 (2013) 083 [arXiv:1302.7205 [hep-th]].

[148] R.-G. Cai, L. Li, L.-F. Li and Y.-Q. Wang, "Competition and Coexistence of Order Parameters in Holographic Multi-Band Superconductors," arXiv:1307.2768 [hep-th].

[149] W.-Y. Wen, M.-S. Wu and S.-Y. Wu, "A Holographic Model of Two-Band Superconductor," arXiv:1309.0488 [hep-th].

[150] Z.-Y. Nie, R.-G. Cai, X. Gao and H. Zeng, "Competition between the s-wave and p-wave superconductivity phases in a holographic model," arXiv:1309.2204 [hep-th].

[151] A. Amoretti, A. Braggio, N. Maggiore, N. Magnoli and D. Musso, "Coexistence of two vector order parameters: a holographic model for ferromagnetic superconductivity," arXiv:1309.5093 [hep-th].

[152] A. Donos, J. P. Gauntlett and C. Pantelidou, "Competing p-wave orders," arXiv:1310.5741 [hep-th].

[153] Y.-i. Shin, C. H. Schunck, A. Schirotzek, and W. Ketterle, "Phase diagram of a twocomponent Fermi gas with resonant interactions," Nature 451 (2008) 689693.

[154] L.-y. He, M. Jin and P.-f. Zhuang, "Pion superfluidity and meson properties at finite isospin density," Phys. Rev. D 71 (2005) 116001 [hep-ph/0503272]; M. N. Chernodub and A. S. Nedelin, "Phase diagram of chirally imbalanced QCD matter," Phys. Rev. D 83 (2011) 105008 [arXiv:1102.0188 [hep-ph]].

[155] R. Combescot, "Introduction to FFLO phases and collective mode in the BEC-BCS crossover", arXiv:cond-mat/0702399v1 [cond-mat.supr-con].

[156] P. Fulde and R. A. Ferrell, "Superconductivity in a Strong Spin-Exchange Field", Phys. Rev. 135 (1964) A550; A. I. Larkin and Y. N. Ovchinnikov, "Nonuniform state of superconductors", ZhEFT, 47 (1964) 1136 [Sov. Phys. JETP, 20 (1965) 762].

[157] F. Bigazzi, A. L. Cotrone, D. Musso, N. P. Fokeeva and D. Seminara, "Unbalanced Holographic Superconductors and Spintronics," JHEP 1202 (2012) 078 [arXiv:1111.6601 [hep-th]].

[158] J. Erdmenger, V. Grass, P. Kerner and T. H. Ngo, "Holographic Superfluidity in Imbalanced Mixtures," JHEP 1108 (2011) 037 [arXiv:1103.4145 [hep-th]].

[159] A. Krikun, V. P. Kirilin and A. V. Sadofyev, "Holographic model of the $S^{ \pm}$multiband superconductor," JHEP 1307 (2013) 136 [arXiv:1210.6074 [hep-th]]. 
[160] I. Amado, D. Arean, A. Jimenez-Alba, K. Landsteiner, L. Melgar and I. S. Landea, "Holographic Superfluids and the Landau Criterion," arXiv:1307.8100 [hep-th].

[161] V. P. Gusynin, V. A. Miransky and I. A. Shovkovy, "Spontaneous rotational symmetry breaking and roton - like excitations in gauged sigma model at finite density," Phys. Lett. B 581 (2004) 82 [hep-ph/0311025].

[162] L. Landau, and M. E. Lifshitz, "Course on Theoretical Physics; Vol 5," Elsevier, 2008.

[163] D. Pines, and P. Nozières, "The Theory of Quantum Liquids," Perseus Books, 1999.

[164] D. Arean, P. Basu and C. Krishnan, "The Many Phases of Holographic Superfluids," JHEP 1010 (2010) 006 [arXiv:1006.5165 [hep-th]].

[165] H. Watanabe, T. áŽBrauner and H. Murayama, "Massive Nambu-Goldstone Bosons," Phys. Rev. Lett. 111 (2013) 021601 [arXiv:1303.1527 [hep-th]].

[166] A. Nicolis, R. Penco, F. Piazza and R. A. Rosen, "More on gapped Goldstones at finite density: More gapped Goldstones," arXiv:1306.1240 [hep-th].

[167] V. Keranen, E. Keski-Vakkuri, S. Nowling and K. P. Yogendran, "Solitons as Probes of the Structure of Holographic Superfluids," New J. Phys. 13 (2011) 065003 [arXiv:1012.0190 [hep-th]].

[168] M. G. Alford, S. K. Mallavarapu, A. Schmitt and S. Stetina, "From a complex scalar field to the two-fluid picture of superfluidity," Phys. Rev. D 87 (2013) 6, 065001 [arXiv:1212.0670 [hep-ph]].

[169] M. G. Alford, S. K. Mallavarapu, A. Schmitt and S. Stetina, "Role reversal in first and second sound in a relativistic superfluid," arXiv:1310.5953 [hep-ph].

[170] D. T. Son, "Low-energy quantum effective action for relativistic superfluids," hep$\mathrm{ph} / 0204199$.

[171] S. Nakamura, H. Ooguri and C.-S. Park, "Gravity Dual of Spatially Modulated Phase," Phys. Rev. D 81 (2010) 044018 [arXiv:0911.0679 [hep-th]].

[172] A. Donos and J. P. Gauntlett, "Holographic striped phases," JHEP 1108 (2011) 140 [arXiv:1106.2004 [hep-th]].

[173] C. A. B. Bayona, K. Peeters and M. Zamaklar, "A Non-homogeneous ground state of the low-temperature Sakai-Sugimoto model," JHEP 1106 (2011) 092 [arXiv:1104.2291 [hep-th]].

[174] O. Bergman, N. Jokela, G. Lifschytz and M. Lippert, "Striped instability of a holographic Fermi-like liquid," JHEP 1110 (2011) 034 [arXiv:1106.3883 [hep-th]].

[175] D. Arean, A. Farahi, L. A. Pando Zayas, I. S. Landea and A. Scardicchio, "Holographic p-wave Superconductor with Disorder," arXiv:1407.7526 [hep-th].

[176] R. E. Arias and I. S. Landea, "Hydrodynamic Modes of a holographic $p$ - wave superfluid," JHEP 1411 (2014) 047 [arXiv:1409.6357 [hep-th]]. 
[177] I. S. Landea, "Inhomogeneous superfluids," arXiv:1410.7865 [hep-th]. 